\title{
Sekundäre Relaxationen in amorphen Festkörpern
}

\section{Mechanische Spektroskopie an \\ metallischen Gläsern und Copolymeren}

\author{
Dissertation \\ zur Erlangung des Doktorgrades \\ der Mathematisch-Naturwissenschaftlichen Fakultäten \\ der Georg-August-Universität zu Göttingen
}

vorgelegt von

Jörg Hachenberg

aus Holzminden

Göttingen, 2006 
D7

Referent: Prof. Dr. Konrad Samwer

Korreferent: Prof. Dr. Michael Buback

Tag der mündlichen Prüfung: 19.10.2006 


\section{Inhaltsverzeichnis}

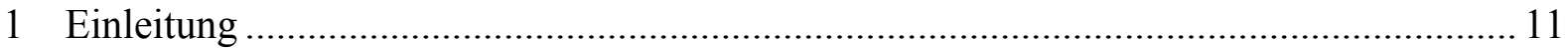

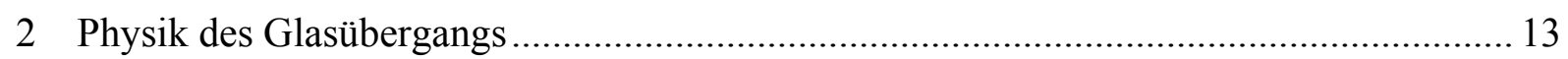

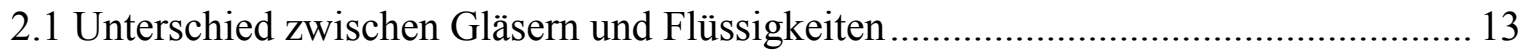

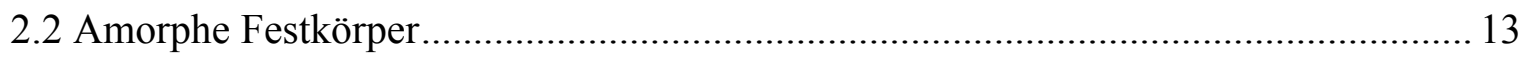

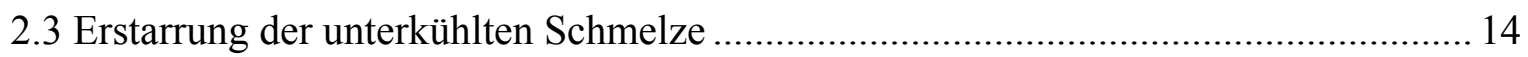

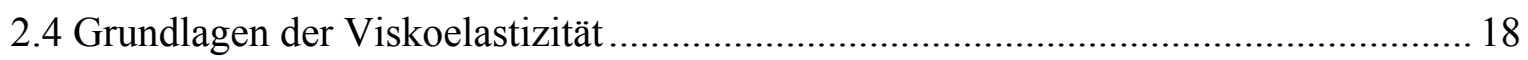

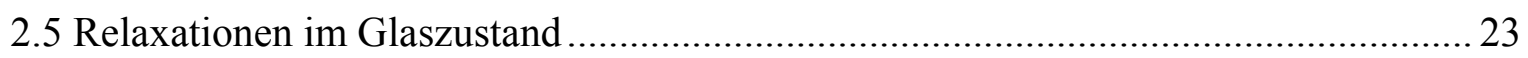

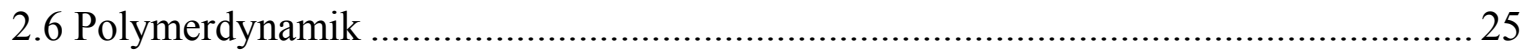

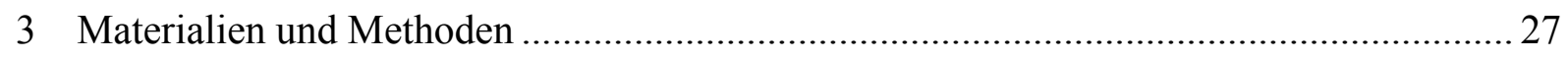

3.1 Präparation von Bändern aus metallischem Glas .................................................... 27

3.2 Radikalische Hochdruck-Copolymerisation von Poly(Ethylen-co-Methacrylsäure)... 29

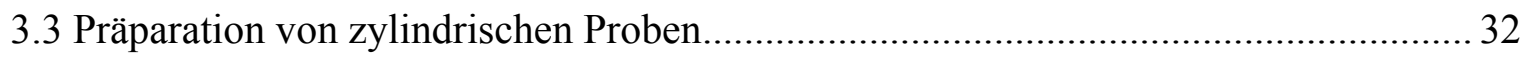

3.4 Rasterelektronenmikroskopie und energiedispersive Röntgen-Mikroanalyse .............. 32

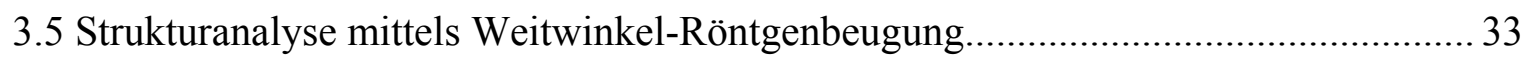

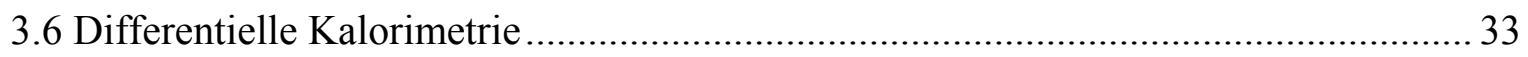

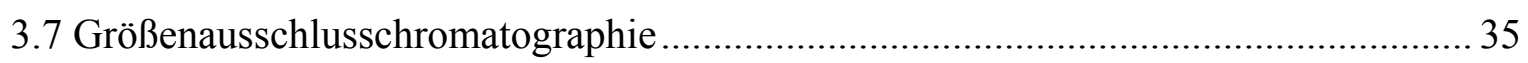

3.8 Bestimmung der Zusammensetzung der Polymerproben........................................... 35

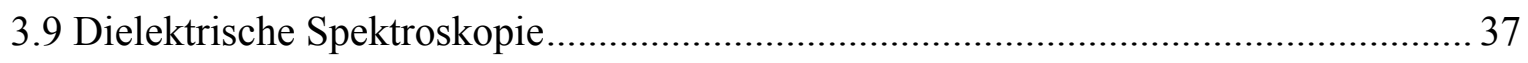

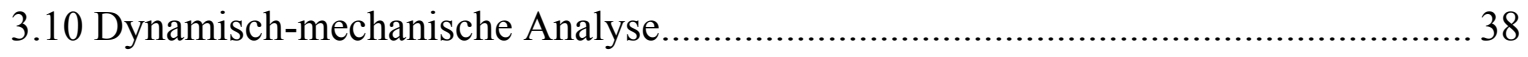

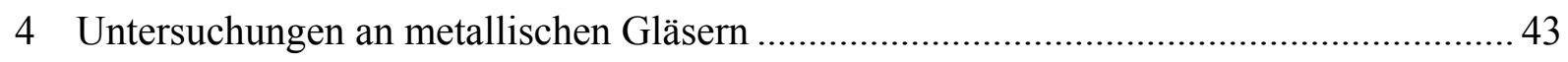

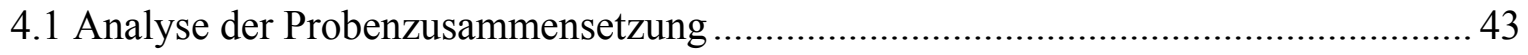

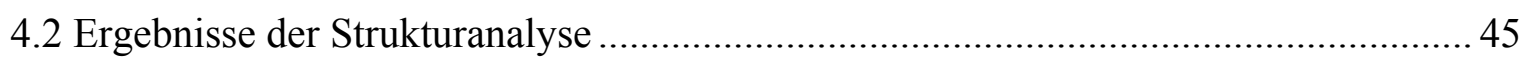

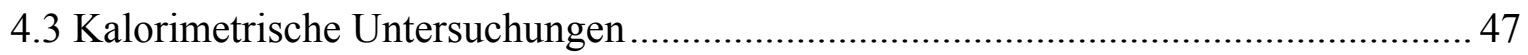

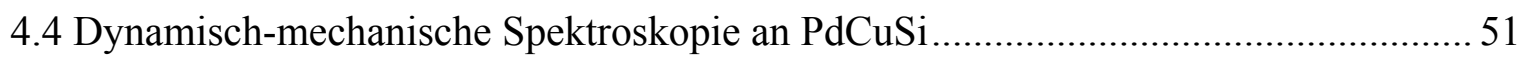

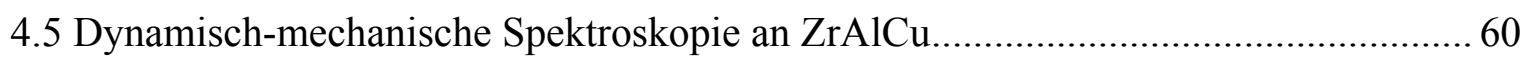

4.6 Verhalten auf großen Temperaturskalen und Alterungsphänomene.............................63

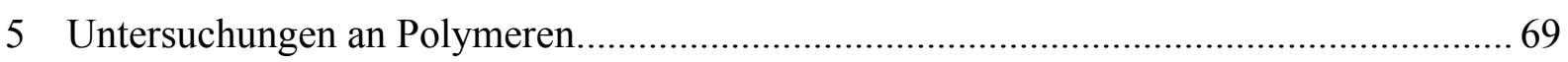

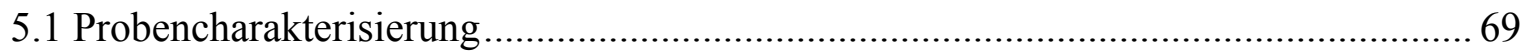

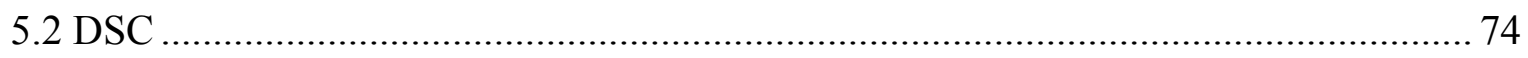

5.3 DMA

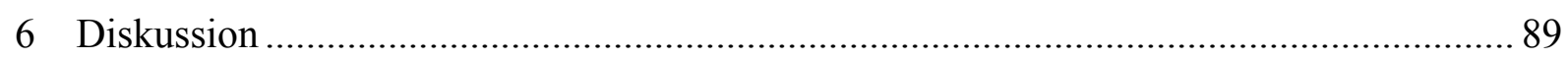

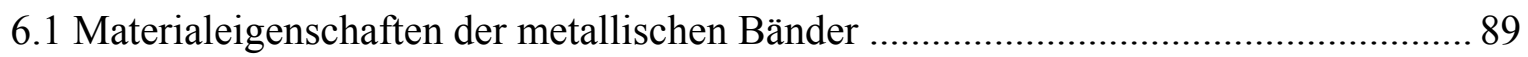

6.2 Sekundäre Relaxationen in metallischen Gläsern ...................................................... 91

6.3 Raten-, Frequenz- und Kraftabhängigkeit der beobachteten Phänomene ..................... 96

6.4 Verhalten auf großen Temperaturskalen und gezieltes Altern ................................... 98

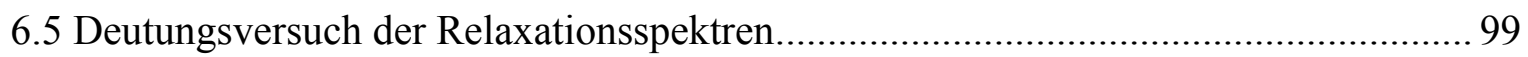

6.6 Sekundäre Relaxationen im Copolymer: Eine Arbeitshypothese ............................. 108

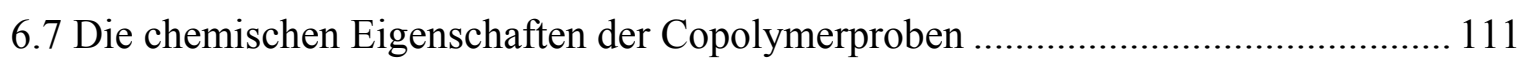

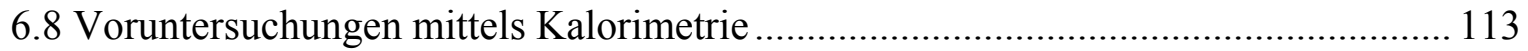

6.9 Mechanische Eigenschaften und „Chemical Confinement“ ...................................... 116 


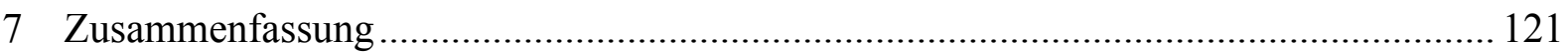

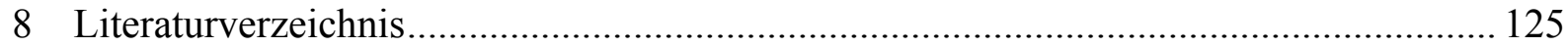

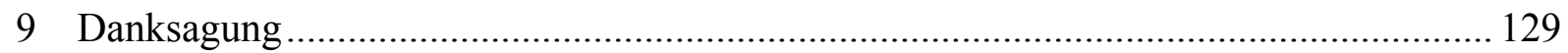

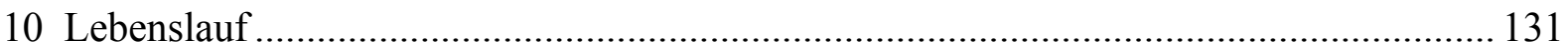




\section{Abbildungsverzeichnis}

Abb. 2.2.1: Verteilung der Atome im a) Gas, b) Kristall, c) Flüssigkeit und c') Glas, schematisch und mit charakteristischer Paarkorrelationsverteilung g(r) [WAS80]

Abb. 2.2.2: Ursprung der Maxima in der Paarkorrelationsfunktion [ELL90]

Abb. 2.3.1: a) Schematische Darstellung der Volumen- oder Enthalpieänderungen beim Kühlen aus der Schmelze [ELL90], b) zugehörige Änderung in der spezifischen Wärme [ZAR91]

Abb. 2.3.2: Schematische Darstellung der fiktiven Temperatur als kühlratenabhängige Glastemperatur [ZAR91].

Abb. 2.3.3: Das Kauzmannparadoxon [STI88]

Abb. 2.3.4: Abhängigkeit der fiktiven Temperatur beim Erhitzen eines Glases von der Heizund Kühlrate im Temperaturverlauf a) des Volumens und b) der spezifischen Wärme [ZAR91]

Abb. 2.4.1: Angell-Plot: Temperaturabhängigkeit der Viskosität oberhalb der Glastemperatur 21

Abb. 2.5.1: Lunkenheimer-Loidl-Plot: Schematische Darstellung des Relaxationsspektrums A

Abb. 2.6.1: Kurbelwellenbewegung nach a) Schatzki und b) Boyer ................................... 25

Abb. 3.1.1: Funktionsweise des Melt Spinners [IRÖ04] .................................................... 28

Abb. 3.2.1: Chemische Struktur der Monomereinheiten, eingebaut im Copolymer: a) Ethylen, b) Methacrylsäure .

Abb. 3.2.2: Technisches Schaltbild des kontinuierlich arbeitenden Hochdruck-Hoch-

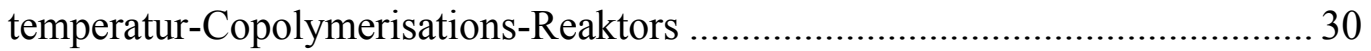

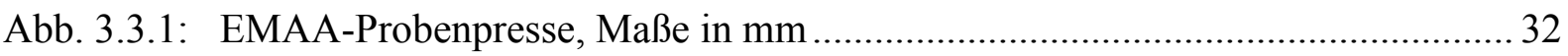

Abb. 3.6.1: Schematischer Aufbau der DSC [WEI98] ........................................................ 34

Abb. 3.8.1: Schematischer Aufbau eines FTIR-Spektrometers für Filme auf einem Träger mit vorgeschaltetem Michelsoninterferometer................................................ 36

Abb. 3.8.2: Schematischer Aufbau eines ATR-Strahlengangs ............................................. 36

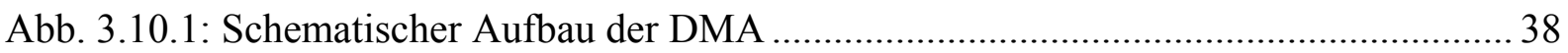

Abb. 3.10.2: Skizze der Kalibrierung mit einem Thermoelement ........................................ 39

Abb. 3.10.3: Schematische Darstellung der Messmodi: a) Uniaxiale Dehnung, b) Kompression, c) Biegebalken mit einseitiger und d) beidseitiger Fixierung [PE94].. 40

Abb. 4.1.1: Beispiel für die Ortswahl der EDX-Analyse anhand der $\mathrm{ZrAlCu}$-Ausgangsprobe 43

Abb. 4.2.1: WAXS an einer PdCuSi-Ausgangsproben, angepasst mit drei Lorentzfunktionen 45

Abb. 4.2.2: WAXS an einer $\mathrm{ZrAlCu}$-Ausgangsprobe, angepasst mit drei Lorentzfunktionen 46

Abb. 4.2.3: WAXS an ausgelagerten PdCuSi- und ZrAlCu-Bändern .................................. 47

Abb. 4.3.1: DSC-Messung bei $5 \mathrm{~K} / \mathrm{min}$ an PdCuSi-Proben unterschiedlicher Masse und

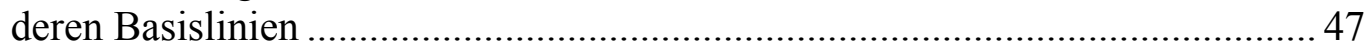

Abb. 4.3.2: DSC-Messung an bei $616 \mathrm{~K}$ ausgelagerten PdCuSi-Proben und einer Referenzmessung.... 48 
Abb. 4.3.3: Ausschnittsvergrößerung mit Wendepunkten und Tangenten zur Bestimmung des Onsets.

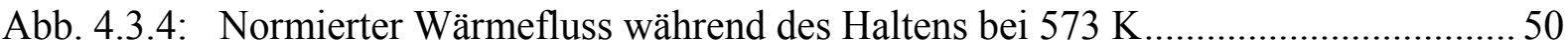

Abb. 4.3.5: Auswirkungen des Haltens bei $573 \mathrm{~K}$ auf die DSC-Messungen......................... 50

Abb. 4.4.1: Rohdaten einer DMA-Messung an PdCuSi bei $1 \mathrm{~Hz}$ und $5 \mathrm{~K} / \mathrm{min}$, Achsenbeschriftungen farbkodiert ....................................................................... 51

Abb. 4.4.2: Verlauf von Speicher- und Verlustmodul einer DMA-Messung bei $1 \mathrm{~Hz}$ und

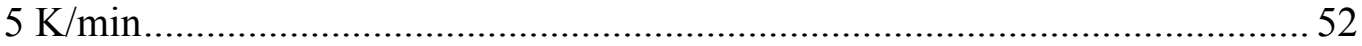

Abb. 4.4.3: Cole-Cole-Fit einer DMA-Messung an PdCuSi bei $1 \mathrm{~Hz}$ und $5 \mathrm{~K} / \mathrm{min}$.............. 53

Abb. 4.4.4: Modifizierter Cole-Cole-Fit einer DMA-Messung an PdCuSi bei $1 \mathrm{~Hz}$ und

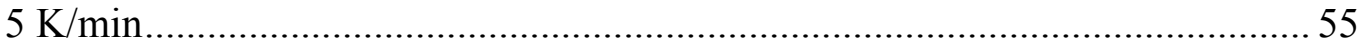

Abb. 4.4.5: Einfluss der Heizrate auf den Phasenwinkel bei der Spektroskopie an PdCuSi . 56

Abb. 4.4.6: Einfluss der Frequenz und der Amplitude auf den Phasenwinkel bei der Spektroskopie an $\mathrm{PdCuSi}$

Abb. 4.4.7: Einfluss der Kraft auf die Viskosität bei der Spektroskopie an PdCuSi bei $1 \mathrm{~Hz}$

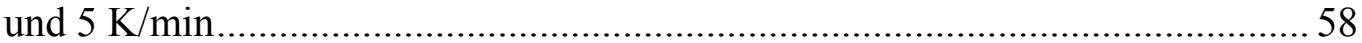

Abb. 4.4.8: Einfluss der Kraft auf die Viskosität bei der Spektroskopie an PdCuSi bei $1 \mathrm{~Hz}$

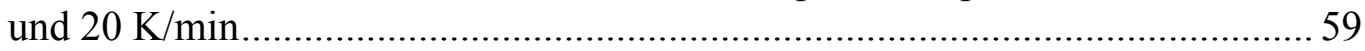

Abb. 4.4.9: Lineare Wärmeausdehnung von PdCuSi mit Stahl-Klemme und Quarz-

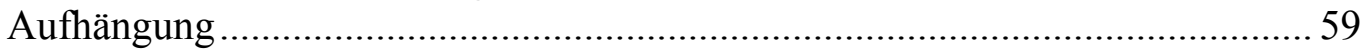

Abb. 4.5.1: Cole-Davidson-Fit einer DMA-Messung an $\mathrm{ZrAlCu}$ bei $1 \mathrm{~Hz}$ und $3 \mathrm{~K} / \mathrm{min}$...... 60

Abb. 4.5.2: Cole-Cole-Fit einer DMA-Messung an $\mathrm{ZrAlCu}$ bei $1 \mathrm{~Hz}$ und $3 \mathrm{~K} / \mathrm{min}$ 61

Abb. 4.5.3: Vergleich von dynamischen Messungen an $\mathrm{ZrAlCu}$ (Achsen: links \& unten) und PdCuSi (Achsen: rechts \& oben, um $48 \mathrm{~K}$ verschoben), die Ordinate ist jeweils auf den Bereich 5\% - $105 \%$ des maximalen Speichermoduls beschränkt........ 62

Abb. 4.6.1: Effekt der thermischen und mechanischen Vorbehandlung auf die Elastizitätsmoduln von PdCuSi ....................................................................... 64

Abb. 4.6.2: Effekt der thermischen und mechanischen Vorbehandlung auf die Elastizitätsmoduln von $\mathrm{ZrAlCu}$

Abb. 4.6.3: Effekt der thermischen und mechanischen Vorbehandlung auf die Verlustmoduln von $\mathrm{ZrAlCu}$ und $\mathrm{PdCuSi}$, verglichen mit Referenzmessungen an Ausgangsproben bei $3 \mathrm{~K} / \mathrm{min}$.

Abb. 4.6.4: Effekt der thermischen und mechanischen Vorbehandlung auf die Verlustmoduln von $\mathrm{ZrAlCu}$ und $\mathrm{PdCuSi}$, verglichen mit Referenzmessungen an Ausgangsproben bei $20 \mathrm{~K} / \mathrm{min}$

Abb. 4.6.5: Effekt der thermischen und mechanischen Vorbehandlung auf die Elastizitätsmoduln von PdCuSi bei einer höheren Frequenz von $5 \mathrm{~Hz}$

Abb. 4.6.6: Anstieg im Speichermodul von PdCuSi bei Heizraten von $0,5 \mathrm{~K} / \mathrm{min}$ und $20 \mathrm{~K} / \mathrm{min}$ und Verlauf der zugehörigen $\tan \delta$.

Abb. 4.6.7: Anstieg im Speichermodul von $\mathrm{ZrAlCu}$ bei Heizraten von $0,5 \mathrm{~K} / \mathrm{min}$ und $20 \mathrm{~K} / \mathrm{min}$ und Verlauf der zugehörigen $\tan \delta$

Abb. 5.1.1: FTIR-Spektrum in Absorption dreier EMAA-Folien mit stark unterschiedlichen Syntheseparametern

Abb. 5.1.2: ATR-FTIR-Spektrum zweier EMAA-Proben mit hohem MAA-Gehalt ............ 71

Abb. 5.1.3: NMR-Spektrum zweier EMAA-Proben der dritten Syntheseserie 
Abb. 5.1.4: MAA-Gehalt der untersuchten Proben in Abhängigkeit der Synthese.bedingungen (Angabe des Synthesedrucks in bar) ......................................... 72

Abb. 5.1.5: Einfluss der Initiatorfördermenge auf den Umsatz der Synthese....................... 73

Abb. 5.1.6: Molmassenverteilung der hergestellten Proben in Abhängigkeit der

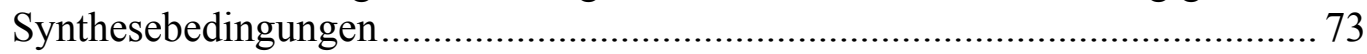

Abb. 5.1.7: Vergleich der Molmassenverteilungen mit den industriellen Proben ................. 74

Abb. 5.2.1: DSC-Messung bei $5 \mathrm{~K} / \mathrm{min}$ an den Homopolymeren PE und PMAA. ............... 75

Abb. 5.2.2: DSC-Messung bei $5 \mathrm{~K} / \mathrm{min}$ an EMAA mit niedrigem MAA-Gehalt und stark

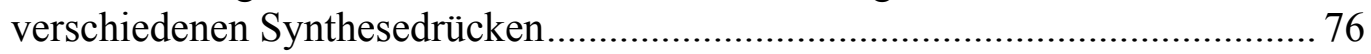

Abb. 5.2.3: DSC-Messung bei $5 \mathrm{~K} / \mathrm{min}$ an EMAA mit mittlerem MAA-Gehalt und stark

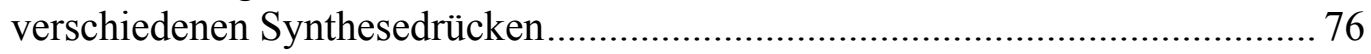

Abb. 5.2.4: DSC-Messung bei $5 \mathrm{~K} / \mathrm{min}$ an EMAA mit hohem MAA-Gehalt und stark verschiedenen Synthesedrücken. .77

Abb. 5.2.5: DSC-Messung bei $5 \mathrm{~K} / \mathrm{min}$ an industriellem EMAA mit unbekannten Syntheseparametern ..................................................................... 78

Abb. 5.2.6: Vergleich der charakteristischen Temperaturen in Abhängigkeit der Molmasse 78

Abb. 5.2.7: Stufenweises Aufheizen bei $5 \mathrm{~K} / \mathrm{min}$ an EMAA mit hohem MAA-Gehalt und hohem Synthesedruck

Abb. 5.2.8: Stufenweises Aufheizen bei $5 \mathrm{~K} / \mathrm{min}$ an EMAA mit hohem MAA-Gehalt und niedrigem Synthesedruck

Abb. 5.3.1: Unterschiede bei der mehrfachen mechanischen Spektroskopie der bei 2300 bar synthetisierten EMAA-Probe mit hohem MAA-Gehalt..... 81

Abb. 5.3.2: Unterschiede bei der mehrfachen mechanischen Spektroskopie der bei 1300 bar synthetisierten EMAA-Probe mit hohem MAA-Gehalt..... 82

Abb. 5.3.3: Verlauf des tan $\delta$ bei der mechanischen Spektroskopie an PE und mit Wasser versetztem PMAA

Abb. 5.3.4: Verlauf des $\tan \delta$ bei der mechanischen Spektroskopie der bei stark verschiedenen Drücken synthetisierten EMAA-Probe mit niedrigem MAAGehalt ..... 84

Abb. 5.3.5: Verlauf des $\tan \delta$ bei der mechanischen Spektroskopie der bei stark verschiedenen Drücken synthetisierten EMAA-Probe mit mittlerem MAAGehalt ..... 84

Abb. 5.3.6: Verlauf des $\tan \delta$ bei der mechanischen Spektroskopie der bei stark verschiedenen Drücken synthetisierten EMAA-Probe mit hohem MAA-Gehalt85

Abb. 5.3.7: Verlauf des tan $\delta$ bei der mechanischen Spektroskopie der industriellen, bei unbekannten Drücken synthetisierten EMAA-Probe mit hohem MAA-Gehalt . 85

Abb. 5.3.8: Vergleich der charakteristischen Temperaturen in Abhängigkeit des MAAGehalts.

Abb. 5.3.9: Ausschluss des systematischen Zusammenhangs zwischen Temperatur der $\alpha-$ Relaxation und der Molmasse in den Copolymerproben

Abb. 6.2.1: Temperaturabhängigkeit der Relaxationszeiten von $\alpha$-, slow $\beta$ - und fast $\beta$ Relaxation von Orthoterphenyl [EAN96], eingetragen sind die Resonanzen $\omega=\tau^{-1}$ (vgl. Kap.2, Gleichung (18))

Abb. 6.2.2: Erstes Indiz für die Existenz eines „excess wing“ aus [RÖS04]....................... 92

Abb. 6.2.3: Kriterium für den Beginn der Kristallisation (Messrate: 20 Datenpunkte/s) zur Messung an $\mathrm{ZrAlCu}$ in Abb. 4.5.1. 
Abb. 6.2.4: Separation des $\beta$-Prozesses aus dem Verlustspektrum von PdCuSi (vgl. Abb. 4.4.4)

Abb. 6.5.1: Der Verlauf des $\tan \delta$ zeigt erst Relaxationseffekte, wenn die Probe deformiert wird (vgl. Abb. 4.6.5). 101

Abb. 6.5.2: Anstieg im Speichermodul von PdCuSi bei Heizraten von $0,5 \mathrm{~K} / \mathrm{min}$ und $20 \mathrm{~K} / \mathrm{min}$ und Verlauf der zugehörigen $\tan \delta$, identisch zu Abb. 4.6.6. 104

Abb. 6.5.3: Heizratenabhängigkeit der Temperaturen des Auftretens von $\alpha$ - und $\beta$ Relaxation bei PdCuSi 106

Abb. 6.5.4: Heizratenabhängigkeit der Temperaturen des Auftretens von $\alpha$ - und $\beta$ Relaxation bei $\mathrm{ZrAlCu}$ 106

Abb. 6.6.1: Eine nicht statistische Verteilung der MAA-Einheiten in EMAA führt zu zusätzlichen Relaxationsmoden. Schematische Darstellung von: a) EMAA mit statistischer MAA-Verteilung, b) mit nicht-statistischer Verteilung, c) der Netzwerkstruktur von EMAA mit statistischer MAA-Verteilung und d) mit nicht statistischer MAA-Verteilung und der daraus resultierenden „Chemical Confinement"-Relaxation 110

Abb. 6.9.1: Auftreten des Alterungseffekt in Sondenposition und Amplitude; Wendepunkt im Amplitudensignal (schwarze Linie) fällt nicht mit jenem des Positionssignals zusammen.

Abb. 6.9.2: Relative Position von statischen und dynamischen Größen der Messung in Abb. 5.3.4..... 


\section{Tabellenverzeichnis}

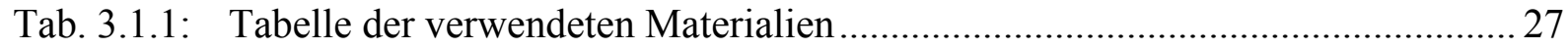

Tab. 3.1.2: Tabelle der Herstellungsparameter beim Melt Spinning...................................... 28

Tab. 3.2.1: Tabelle der Polymerisationsparameter ................................................................ 31

Tab. 4.1.1: Auflistung der Ergebnisse der EDX-Analyse an drei unterschiedlich vorbehandelten PdCuSi-Proben

Tab. 4.1.2: Auflistung der Ergebnisse der EDX-Analyse an drei unterschiedlich vorbehandelten $\mathrm{ZrAlCu}$-Proben $44 \mathrm{M}$

Tab. 4.3.1: Tabelle der Onsets und Wendepunkte der DSC-Messungen bei $5 \mathrm{~K} / \mathrm{min}$ 49

Tab. 4.4.1: Parameter der Cole-Cole-Anpassung einer DMA-Messung an PdCuSi bei $1 \mathrm{~Hz}$

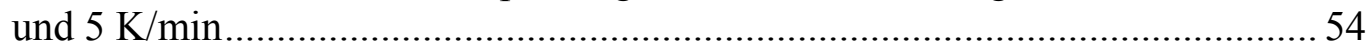

Tab. 4.4.2: Parameter der modifizierten Cole-Cole-Anpassung einer DMA-Messung an

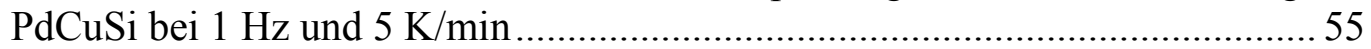

Tab. 4.4.3: Heizratenabhängigkeit der Onsets der tan $\delta$-Maxima bei der Spektroskopie an PdCuSi mit Korrektur aus [RAM94] …...................................................... 56

Tab. 4.4.4: Einfluss der Frequenz und der Amplitude auf den Onset von tan $\delta$.................. 57

Tab. 4.5.1: Parameter der Cole-Davidson-Anpassung einer DMA-Messung an ZrAlCu bei

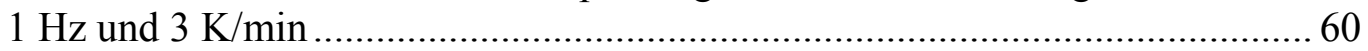

Tab. 4.5.2: Parameter der Cole-Cole-Anpassung einer DMA-Messung an ZrAlCu bei $1 \mathrm{~Hz}$

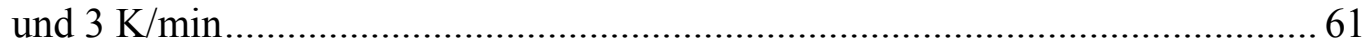

Tab. 5.1.1: Kalibration der quantitativen FTIR-Analyse nach [WITT99] ............................ 70

Tab. 5.1.2: Tabelle der Polymerisationsparameter (identisch zu Tab. 3.2.1) ........................ 72 


\section{Einleitung}

Der Begriff „Glas“ bezieht sich umgangssprachlich auf eine Menge von Materialien, die sich dadurch auszeichnen, fest und transparent zu sein, wie zum Beispiel Fensterglas oder Plexiglas. Die hier verwendete Definition eines Glases ist aber allgemeiner: Es handelt sich um ein Glas, wenn das Material ein amorpher Festkörper ist, der einen Glasübergang zeigt [ELL90]. „Amorph“ leitet sich aus dem Griechischen für ,gestaltlos“ ab und bedeutet, dass es, im Gegensatz zum kristallinen Zustand, keine langreichweitige Ordnung der Atom- oder Molekülpositionen im Festkörper gibt. Dies ist auch bei Fensterglas und vielen Kunststoffen der Fall, aber auch bei ganz anderen Materialien wie zum Beispiel den metallischen Gläsern. Diese sind aber im Gegensatz zu Fensterglas undurchsichtig, elastisch und leiten den elektrischen Strom.

Die zweite Eigenschaft, die ein Glas ausmacht, ist einen Glasübergang zu zeigen. Darunter wird verstanden, dass es möglich ist, das Material durch eine Änderung einer Zustandsgröße, wie Temperatur, Druck oder Zusammensetzung, in den flüssigen Zustand zu überführen. Dies wird klar am Beispiel der Glasbläserei: Bei Zimmertemperatur ist Silikatglas fest, während es sich bei hohen Temperaturen formen lässt und sich bei noch höheren Temperaturen wie eine Flüssigkeit verhält. Aber auch die Beobachtungszeitskala ist entscheidend. Dies zeigt sich eindrucksvoll beim Sprung vom Zehnmeterturm: Das unter Normalbedingungen flüssige Wasser ist auf der sehr kurzen Zeitskala des Auftreffens auf die Wasseroberfläche hart und verhält sich wie ein Festkörper. Obgleich es sich also um ein sehr grundlegendes Phänomen handelt, ist die Physik des Glasübergangs bis heute im Detail nicht verstanden [DS01].

Die mechanische Spektroskopie [MEN99] ist zur Untersuchung des Glasübergangs sehr nützlich, weil sehr genau bestimmt werden kann, ob sich die Probe fest wie ein Glas verhält oder fließt. Dazu wird die Probe mit einer externen Frequenz deformiert und beobachtet, ob die Probe der Deformation auf der Zeitskala der Spektroskopiefrequenz folgen kann oder nicht. Ist die Relaxationszeit des Materials viel länger als die Schwingungsdauer, so verhält sich das Glas fest, im umgekehrten Fall ist es flüssig. Um den Glasübergang von fest zu flüssig zu durchlaufen, besteht nun die Möglichkeit bei fester Spektroskopiefrequenz die Relaxationszeit, zum Beispiel durch Temperaturerhöhung, zu verkürzen, oder bei fester Temperatur und konstanter Relaxationszeit die Spektroskopiefrequenz kontinuierlich zu senken. Je nachdem ob der Abfall der Relaxationszeit, der 14 Dekaden überspannen kann, in einem scharfen Temperaturintervall oder einem großen Bereich exponentiell erfolgt, wird von einem fragilen oder starken Glas gesprochen [BNA93]. Aus dem gemessenen Verlauf der mechanischen Eigenschaften lässt sich dann ersehen, wie viele Relaxationsprozesse auftreten. Die Form und die Position der Relaxationen lassen bei Variation der Messparameter Schlüsse auf die Art des Relaxationsprozesses zu.

Im Bereich hoher Temperatur und auf einer Zeitskala von kurzen Relaxationszeiten können das Relaxationsverhalten von Gläsern und die damit verknüpften Größen der Viskosität und der Diffusionskonstante [FFM03] theoretisch gut mit dem Moden-Kopplungs-Modell beschrieben werden [GÖT99]. Bei tieferen Temperaturen und längeren Relaxationszeiten dominieren Bereiche mit einer schnelleren Dynamik das Relaxationsverhalten der gesamten Probe. Es wird dabei von dynamischen Heterogenitäten gesprochen, für deren Beschreibung es eine 
Vielzahl von theoretischen Ansätzen gibt [SIL99]. Trotz der Komplexität der Situation [DS01] konnten kürzlich große Fortschritte in der Beschreibung der den Glasübergang dominierenden, so genannten $\alpha$-Relaxation gemacht werden [JS05, ZSJ06, DHT06].

Darüber hinaus gibt es aber experimentelle Studien [RSL04] und Computersimulationen [TEI05] an metallischen Gläsern, die zeigen, dass eine einzige Relaxationsmode zur Beschreibung des Glasübergangs nicht ausreicht und die Existenz einer zusätzlichen, so genannten langsamen $\beta$-Relaxation postulieren. Gleichartige Beobachtungen sind bei organischen Glasbildnern keine Seltenheit und wurden bereits detailliert untersucht [LL00]. Organische Glasbildner und insbesondere Polymere verfügen aber über eine im Vergleich zu metallischen Gläsern deutlich kompliziertere molekulare Struktur, die zu zusätzlichen Relaxationsmoden führen kann [MRW67, ZOR03]. Um ein tieferes Verständnis der Relaxationsprozesse zu gewinnen, ist es also wichtig, möglichst einfache Systeme zu untersuchen, oder in Folge einer gezielten Manipulation der Geometrie [SK03, AM05, SGS05] die Auswirkungen im Relaxationsspektrum zu untersuchen. Beide Ansätze werden in dieser Arbeit verfolgt.

Die metallischen Gläser sind besonders einfach aufgebaute amorphe Festkörper, weil sie als System harter Kugeln mit attraktiven und repulsiven Wechselwirkungen beschrieben werden können [FFM03]. Es gibt somit keine störenden materialspezifischen Relaxationsmoden. Nichtsdestotrotz gibt es auch bei den metallischen Gläsern solche, deren Relaxationsverhalten ein eher starkes oder ein eher fragiles Verhalten zeigen. Erste Indizien für die Existenz einer langsamen $\beta$-Relaxation konnten im eher starken Glasbildner $\mathrm{Zr}_{65} \mathrm{Al}_{7,5} \mathrm{Cu}_{27,5}$ bereits nachgewiesen werden [RSL04]. Es gilt in dieser Arbeit auch eine eher fragile amorphe Legierung, $\mathrm{Pd}_{77,5} \mathrm{Cu}_{6} \mathrm{Si}_{16,5}$, zu untersuchen. Gelingt es auch hier die langsame $\beta$-Relaxation nachzuweisen, so rechtfertigt dies, von einem für den Glasübergang universellen Phänomen zu sprechen. Darüber hinaus zeigen metallische Gläser beim ersten Aufheizen Alterungseffekte, die es gilt mit dem bestehenden Relaxationsspektrum in Einklang zu bringen, oder dieses zu ergänzen.

Bei Polymeren resultiert die Erweichung am Glasübergang aus einer Segmentbewegung [MRW67]. Wird die Segmentbeweglichkeit durch bei tiefen Temperaturen feste Knotenpunkte eingeschränkt, so sollte dies das Auftreten der zugehörigen Relaxationsmode beeinflussen oder zu weiteren Relaxationsmoden bei höheren Frequenzen bzw. tieferen Temperaturen führen. Für diese Versuche ist Poly(Ethylen-co-Methacrylsäure) (EMAA) ein hervorragender Versuchskandidat. Es handelt sich dabei um ein Copolymer, dass aus schwach wechselwirkenden Ethylen- und stark untereinander wechselwirkenden Methacrylsäure(MAA)-Einheiten aufgebaut ist. Durch Variation des Synthesedrucks während der radikalischen Polymerisation im kontinuierlich betriebenen Hochdruck-Hochtemperatur-Rührkessel [WITT99] ist es möglich, Einfluss auf die Statistik der MAA-Verteilung entlang der Kette zu nehmen, ohne deren Konzentration signifikant zu verändern. Dies sollte einen starken Einfluss auf die Längenverteilung der zwischen den MAA-Einheiten liegenden Ethylen-Segmente und somit auch deren Schwingungsverhalten haben. Es gilt hier, zu untersuchen, wie diese Manipulationen auf mikroskopischer Skala die makroskopischen mechanischen Eigenschaften beeinflussen und die Änderungen im Relaxationsspektrum zu deuten. 


\section{Physik des Glasübergangs}

Die Physik des Glasübergangs ist sehr umfangreich, und in vielen Punkten basieren die etablierten theoretischen Konzepte auf Näherungen und phänomenologischen Beobachtungen. Deshalb wird in diesem Kapitel nur auf die Grundlagen der Theorie [ZAR91, ELL90, DON01] und die für die Spektroskopie relevanten Themen eingegangen werden.

\subsection{Unterschied zwischen Gläsern und Flüssigkeiten}

Die beiden wesentlichen Parameter, die ein Glas von einer Flüssigkeit unterscheiden, sind die Temperatur und die Zeitskala bzw. die Frequenz bei der Beobachtung des Materials. Die Deborah Zahl DE bietet dabei die einfachste und wohl auch treffendste Unterscheidungsmöglichkeit [MEN99]:

$$
D E=\frac{\tau}{t_{c h a r}}
$$

Je kleiner das Verhältnis von der Relaxationszeit $\tau$ des Materials zu der für den Versuch charakteristischen Beobachtungszeit $t_{\text {char }}$ ist, umso flüssiger erscheint das Material. Der Glaszustand liegt vor, wenn die Relaxationszeit viel größer ist als die charakteristische Zeit. Das Material erscheint dann auf der Beobachtungszeitskala fest. Die Relaxationszeit kann dabei sehr stark temperaturabhängig sein, worauf im Folgenden noch näher eingegangen wird.

\subsection{Amorphe Festkörper}

Nach der vorangegangenen Definition gehören zu der Menge der Gläser weitaus mehr Materialien als nur das Fensterglas. Ein Glas ist nicht zwangsläufig transparent und spröde, sondern kann z. B. auch metallisch und elastisch sein. Nach [ELL90] ist ein Glas „, ein amorpher Festkörper, der einen Glasübergang zeigt.“ Amorph beschreibt dabei, dass die Atompositionen im Glas keine Fernordnung besitzen. Der Zustand im Glas entspricht damit viel eher dem in der Flüssigkeit:
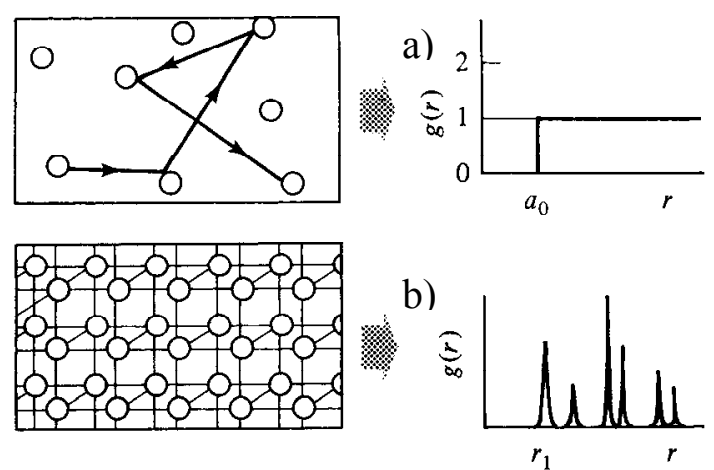

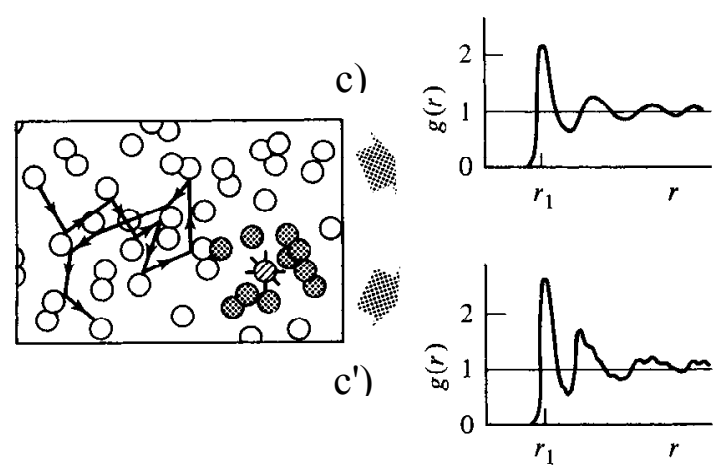

Abb. 2.2.1: Verteilung der Atome im a) Gas, b) Kristall, c) Flüssigkeit und c') Glas, schematisch und mit charakteristischer Paarkorrelationsverteilung g(r) [WAS80]

Abbildung 2.2.1 zeigt deutlich die Unterschiede in der Atomanordnung zwischen einem Gas a) und einem Kristall b), wobei hier das Material vereinfacht aus harten Kugeln mit attraktiver Wechselwirkung besteht und Überschneidungen der Kugeln ausgeschlossen sind. Im Gas können sich die Atome frei bewegen und interagieren durch elastische Stöße. Die Geschwin- 
digkeiten besitzen dabei eine Maxwell-Verteilung, welche die Temperatur des Systems widerspiegelt [GV97]. Die Wahrscheinlichkeit, ein zweites Atom im Abstand $r$ zum ersten zu finden, gemittelt über die Zeit und alle Teilchen, gibt die Paarkorrelationsfunktion $g(r)$ an. Die Mittelpunkte zweier Kugeln können sich nicht näher kommen als die Summe ihrer Radien. Deshalb ist $g(r)=0$ bis der Abstand den Durchmesser $a_{0}$ erreicht und ist danach im Gas konstant, weil es keine bevorzugten Aufenthaltsorte gibt. Der Wert für $r>a_{0}$ ist abhängig von der Dichte des Materials. Der Einfachheit halber ist $g(r)$ in Abb. 2.2.1 a), c) und c') auf diesen tatsächlichen Wert $g^{\prime}(\infty)$ normiert und somit gleich 1. Im idealen Kristall b) sitzen alle Atome auf festen Gitterplätzen. $g(r)$ besteht deshalb aus scharfen Maxima, welche den Abständen im Kristallgitter entsprechen. Ist der Kristall nicht perfekt, so ist die Breite der Maxima abhängig von der Unordnung im System.

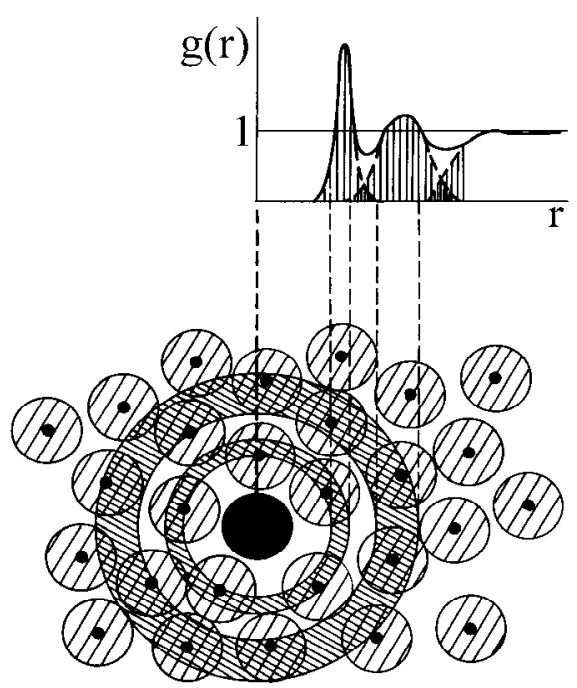

Abb. 2.2.2: Ursprung der Maxima in der Paarkorrelationsfunktion [ELL90]

Deutlich schwieriger ist nun der Unterschied zwischen einer Flüssigkeit und einem Glas. Sowohl in der Flüssigkeit als auch im Glas gibt es eine Nahordnung. Diese kann chemischer Natur sein, wenn mehrere Atomsorten vorhanden sind oder sich auf die Entstehung eines bevorzugten Abstandes zum nächsten Nachbarn beschränken. Es gibt jedoch keine Fernordnung. Dies führt zu breiten Maxima der Paarkorrelationsfunktion $g(r)$, welche aus der Bildung von Koordinationsschalen resultiert (Abb. 2.2.2) [JAN94]. Die Fläche unter den Maxima ist ein Maß für die effektive Koordinationszahl [ELL90], also die mittlere Anzahl der Nachbarn in der entsprechenden Schale. Die Paarkorrelationsfunktion zeigt bei Gläsern kleine Unterschiede im Vergleich zur Flüssigkeit (Abb. 2.2.1 c) und c')). Diese beruhen darauf, dass die Position des übernächsten Nachbarn in der Flüssigkeit durch thermische Bewegungen stärker verschmiert ist, und im Glas noch etwas mehr Struktur zu erkennen ist. So können diese übernächsten Nachbarn einfach den Abstand 2 $a_{0}$ haben, wenn ein Atom genau zwischen ihnen liegt. Oder sie befinden sich in einer Ebene an den entfernten Ecken zweier aneinander gefügter, gleichseitiger Dreiecke (Abstand $3^{0,5} \cdot a_{0} \approx 1,73 \cdot a_{0}$ ), oder aber an den entfernten Ecken zweier aneinander gefügter Tetraeder (Abstand $(8 / 3)^{0,5} \cdot a_{0} \approx 1,63 \cdot a_{0}$ ). Dies sind die einfachsten geometrischen Anordnungen. Es gibt aber in drei Dimensionen eine Vielzahl von Möglichkeiten. Dies kann mit Hilfe einer Kugelschüttung demonstriert werden. Dabei werden Kugeln in ein rundes Gefäß geschüttet und die Zwischenräume mit Klebstoff ausgefüllt. Danach können die geometrischen Formationen (Bernal-Polyeder) klassifiziert und ausgezählt werden [BER60]. Eine Alternative dazu sind Computersimulationen, z. B. [WBZ02].

\subsection{Erstarrung der unterkühlten Schmelze}

\section{Glasübergang}

Die gängigste Methode ein Glas herzustellen ist, es aus der Schmelze abzukühlen. Abbildung 2.3.1 zeigt dabei die möglichen Übergänge des Materials, kommend aus der Flüssigkeit. Beim 
Erreichen der Schmelztemperatur $T_{\mathrm{M}}$ geschieht im thermodynamischen Gleichgewicht eine Materialumwandlung in den kristallinen Zustand. Viele Polymere bilden hier eine Ausnahme, da sie meist amorph vorliegen oder zumindest unvollständig kristallisieren. Die Kristallisation führt, da es sich um einen Phasenübergang erster Ordnung handelt, zu einem Sprung in der Enthalpie $H$ bzw. im Volumen $V$ (Abb. 2.3.1 a)) und somit auch zu einer Unstetigkeit in der spezifischen Wärme (Abb. 2.3.1 b), gestrichelte Linie). Geschieht das Kühlen ausreichend schnell, so lässt sich die Kristallisation unterdrücken; das System liegt dann als unterkühlte Schmelze vor. Streng thermodynamisch betrachtet ist dies bereits ein Nichtgleichgewichtszustand. In der Glasphysik wird hier aber noch häufig von einem Gleichgewicht gesprochen, da der Kurvenverlauf in der unterkühlten Schmelze als Interpolation des Verlaufs in der Schmelze betrachtet werden kann und das Material sich nach wie vor wie eine Flüssigkeit verhält. Kristallisationskeime unter einer kritischen Größe sind in der unterkühlten Schmelze instabil [HAA94]; es gilt also Kristallisationskeime zu vermeiden. Diese können durch thermische Fluktuationen (homogene Keimbildung), eine schlechte Durchmischung, Verunreinigungen oder durch Grenzflächeneffekte entstehen (heterogene Keimbildung).
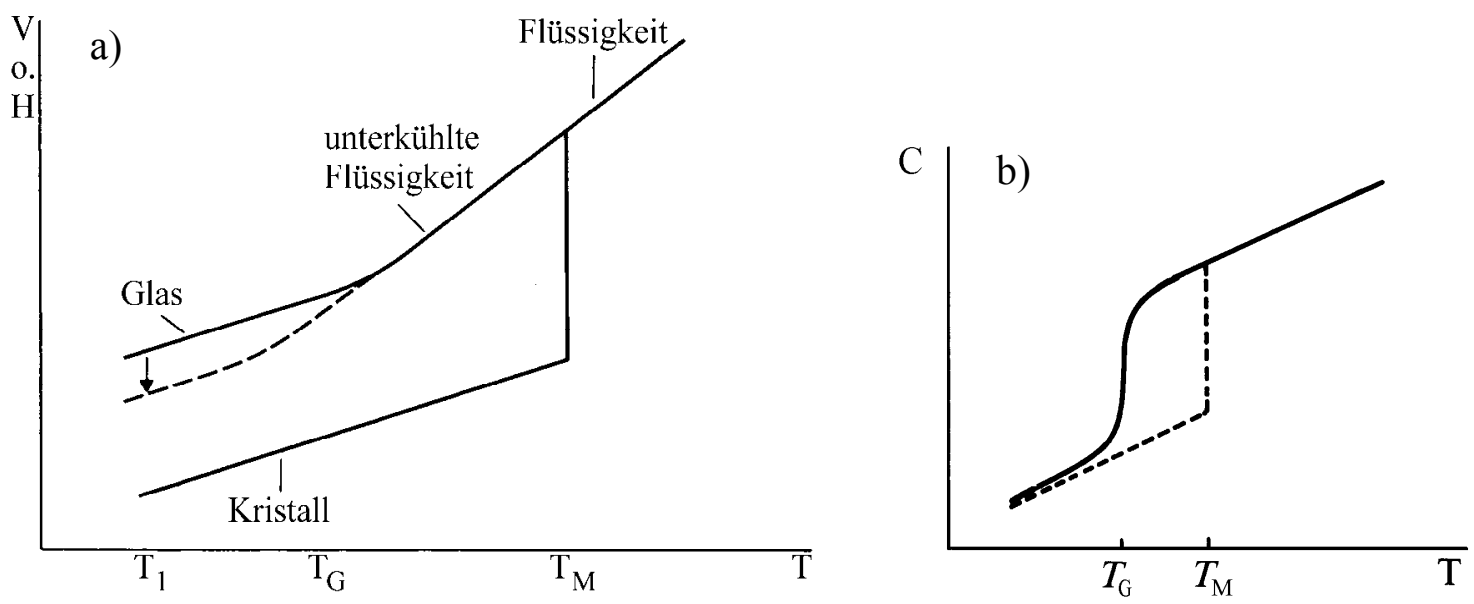

Abb. 2.3.1: a) Schematische Darstellung der Volumen- oder Enthalpieänderungen beim Kühlen aus der Schmelze [ELL90], b) zugehörige Änderung in der spezifischen Wärme [ZAR91] Je weiter die Schmelze abgekühlt wird, umso kleiner ist die kritische Größe eines stabilen und damit wachstumsfähigen Kristallisationskeims; die Dynamik nimmt aber auch weiter ab. Am Glasübergang $T_{\mathrm{G}}$ ist die Dynamik des Systems zu langsam, als dass sie weiter dem Gleichgewichtsverhalten der Flüssigkeit folgen könnte, und das System fällt aus dem (metastabilen) Gleichgewicht. Da die Relaxationszeit jetzt länger ist, als die charakteristische Zeit, in der weiter abgekühlt wird, ist nun die Deborah-Zahl $D E>1$ (Gleichung(1)). Bei gleich bleibender Kühlrate befindet sich das System nun im Glaszustand. Wird der Abkühlvorgang mit einer anderen, niedrigeren Kühlrate durchgeführt, so ist die charakteristische Zeit für das Abkühlen länger. Das System fällt dann erst bei einer niedrigeren Temperatur aus dem Gleichgewicht (gestrichelter Verlauf in Abb. 2.3.1 a)).

Beim Glasübergang kann demnach ein Abknicken der Enthalpie und des Volumens als Funktion der Temperatur beobachtet werden, jedoch kein Sprung wie bei der Kristallisation. Es handelt sich also nicht um einen Phasenübergang erster Ordnung. Dieses Abknicken ist nicht scharf, da die Relaxationszeit nicht auf einem einzelnen Prozess beruht, sondern sich aus vie- 
len Relaxationszeiten der Einzelprozesse zusammensetzt und diese auch miteinander koppeln können [ZAR91]. Deshalb gibt es auch keinen Sprung in der Ableitung beobachtbarer Größen, wie der spezifischen Wärme (Abb. 2.3.1 b)) oder dem thermischen Ausdehnungskoeffizienten. Der Phasenübergang ist folglich auch nicht eindeutig zweiter Ordnung. Die Ordnung des Phasenübergangs ist immer noch Gegenstand der Diskussion. So gibt es Überlegungen, ob das Einfrieren der Dynamik den Übergang nur verschmiert und bei unendlich langsamem Abkühlen ohne auftretende Kristallisation nicht doch ein Phasenübergang zweiter oder gar erster Ordnung beobachtet werden könnte [BK05, AT74, STI88].

\section{Ratenabhängigkeit, fiktive Temperatur und Kauzmann-Paradoxon}

Beim Kühlen aus der Schmelze kann der Glasübergang bei verschiedenen Temperaturen stattfinden. Je nach Wahl der Kühlrate werden unterschiedliche Zustände der unterkühlten Schmelze eingeschreckt. Die Glastemperatur ist also keine universelle Materialkonstante, sondern besitzt nur für die jeweilige Kühlrate Gültigkeit. Die ratenabhängige Glastemperatur ist aber geeignet, den eingeschreckten Zustand zu charakterisieren. Sie wird in diesem Zusammenhang als fiktive Temperatur $T_{F}$ bezeichnet.

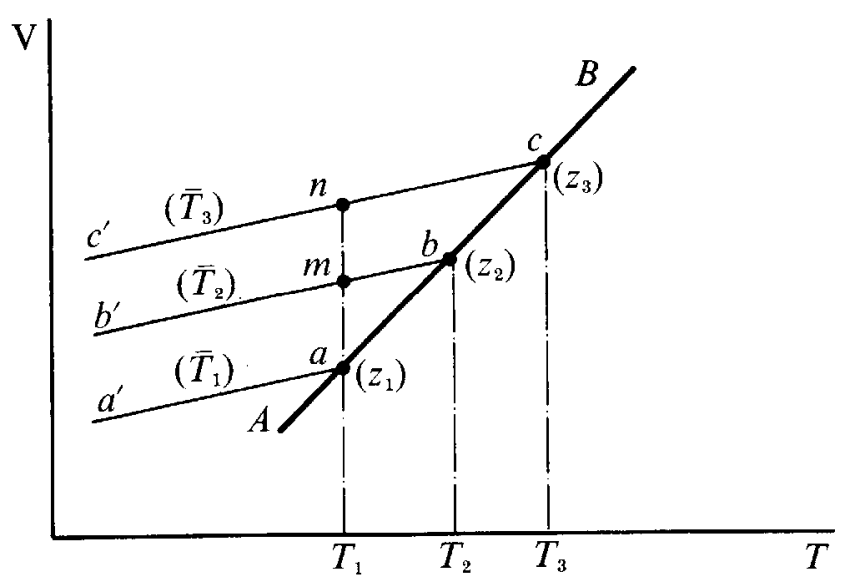

Abb. 2.3.2: Schematische Darstellung der fiktiven Temperatur als kühlratenabhängige Glastemperatur [ZAR91]

Abbildung 2.3.2 illustriert die Zusammenhänge: Bei $T_{1}$ erstarrt das System bei entsprechender Kühlrate in Punkt $a$ aus der Schmelze $A B$ und geht in den Glaszustand $a$ ' über. Es besitzt damit die fiktive Temperatur $\bar{T}_{1}$. Höhere Kühlraten führen zu höheren fiktiven Temperaturen. Bei $T_{l}$ befindet sich das System $b$ ' bzw. $c$ ' im Punkt $m$ bzw. $n$ in einem eingeschreckten $\mathrm{Zu}$ stand mit der fiktiven Temperatur $\overline{T_{2}}$ bzw. $\bar{T}_{3}$.

Die Interpolation der Liquiduslinie $A B$ durch immer langsameres Kühlen unter Vermeidung von Kristallisation führt in Abb. 2.3.3 zu einem Schnittpunkt mit der Linie des Kristalls. Dies bedeutet, dass die Entropie dieses ,,idealen Glases“ denselben Wert wie der Kristall erreichen könnte. Da es unvorstellbar ist, dass ein ungeordneter Festkörper weniger Entropie als ein Kristall besitzt, wird dies als Kauzmann-Paradoxon bezeichnet [STI88]. 


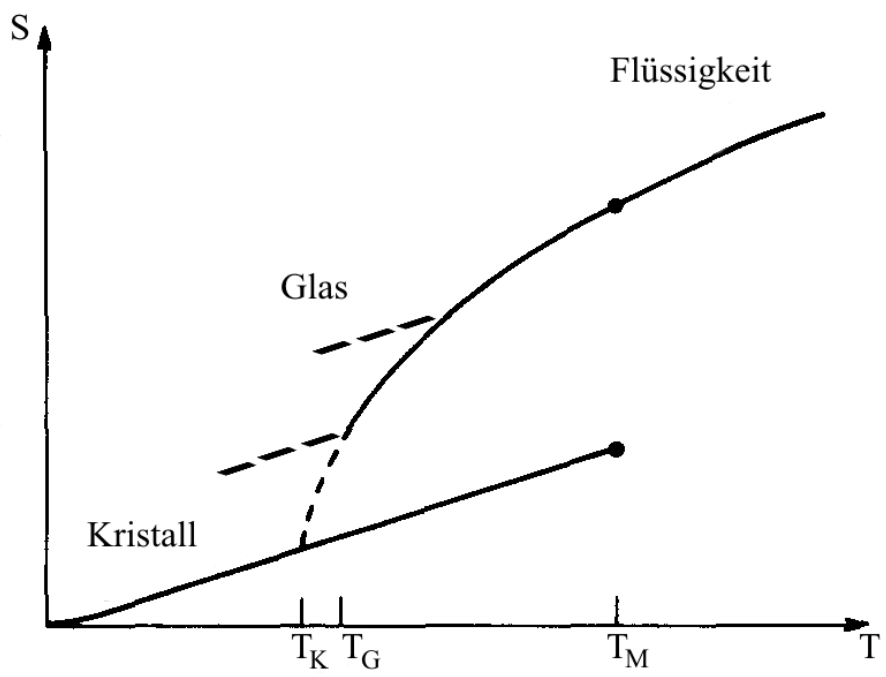

Abb. 2.3.3: Das Kauzmannparadoxon [STI88]

\section{Alterungsprozesse}

Bei Temperaturen, die viel kleiner sind als die Kauzmann-Temperatur $T_{\mathrm{K}}$, bleibt das Glas in guter Nährung fest in seinem eingeschreckten Zustand mit der zugehörigen fiktiven Temperatur $T_{\mathrm{F}}$. Der Zustand ist ebenfalls statisch, wenn sich das System im metastabilen Gleichgewicht der unterkühlten Schmelze befindet, ohne dass eine Kristallisation auftritt. Abbildung 2.3.4 verdeutlicht was passiert, wenn sich die Temperatur im Bereich zwischen $T_{\mathrm{K}}$ und $T_{\mathrm{M}}$ befindet.
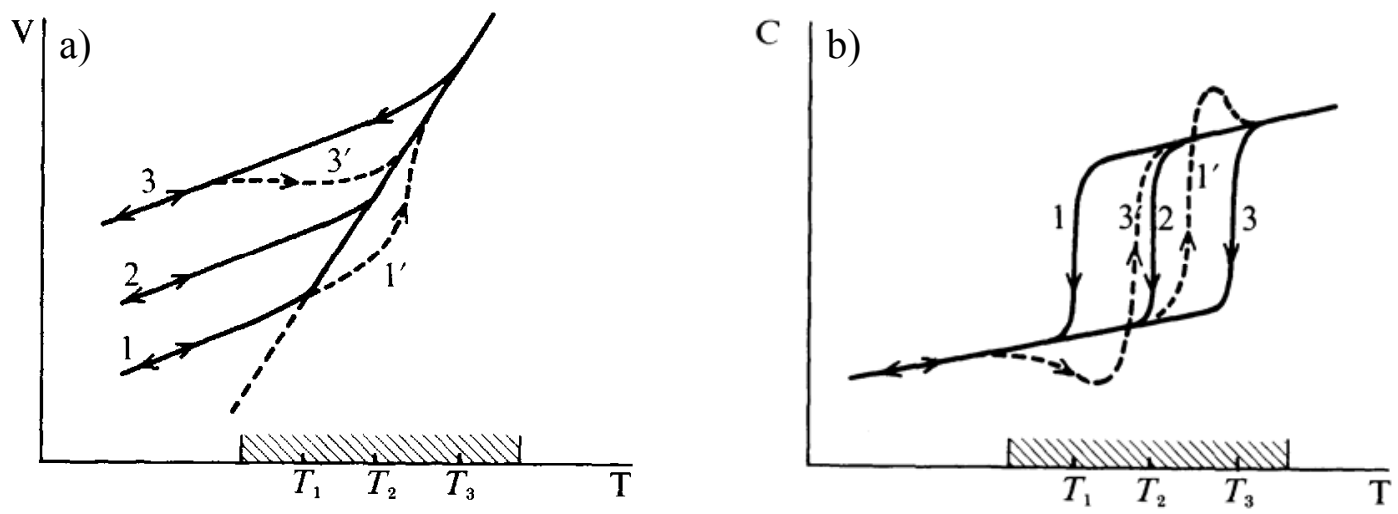

Abb. 2.3.4: Abhängigkeit der fiktiven Temperatur beim Erhitzen eines Glases von der Heizund Kühlrate im Temperaturverlauf a) des Volumens und b) der spezifischen Wärme [ZAR91]

Im einfachsten Fall (1, 2 oder 3) ist die Heizrate gleich der vorangegangenen Kühlrate. Dann knickt der Verlauf des Volumens (Abb. 2.3.4 a)) bei $T_{\mathrm{G}}=T_{\mathrm{F}} \mathrm{ab}$, was zu einem Anstieg in der Wärmekapazität führt (Abb. 2.3.4 b)). Liegt durch langsameres Kühlen anfangs der Zustand $\bar{T}_{1}$ vor und erfolgt das das Heizen nun mit einer schnelleren Rate (Linie 1'), so schießt der Verlauf des Volumens zunächst über die ursprüngliche fiktive Temperatur hinaus und nähert sich dem Verlauf des Volumens in der unterkühlten Schmelze von unten. Der steilere Anstieg 
führt zu einem Maximum in der Wärmekapazität. Im umgekehrten Fall wird langsamer geheizt, als zuvor gekühlt wurde. Linie 3' zeigt, dass sich der Verlauf des Volumens unterhalb des Glasübergangs dem Zustand annähert, welcher der aktuellen Heizrate entsprechen würde, bevor die unterkühlte Schmelze erreicht ist. Dies führt zu einem Minimum in der Wärmekapazität. Tritt keine Kristallisation auf, so kann durch Heizen in die unterkühlte Schmelze und anschließendes Kühlen reversibel zwischen Glaszuständen mit unterschiedlichen fiktiven Temperaturen gewechselt werden.

Komplizierter wird die Situation, wenn das System mit einer von der ursprünglichen Kühlrate verschiedenen Heizrate erhitzt (1' oder 3') und wieder abgekühlt wird, ohne zuvor das metastabile Gleichgewicht der unterkühlten Schmelze erreicht zu haben. Auf diese Weise entsteht ein Mischzustand zwischen den zu den beiden Raten gehörenden Zuständen. Von einem Altern oder ,physical aging“ wird gesprochen, wenn ein zuvor rasch abgeschrecktes System langsam erhitzt und somit teilweise in einen zu einer niedrigeren fiktiven Temperatur gehörenden Zustand überführt wird. Eine Verjüngung entsteht, wenn ein System schnell und kurz über seine fiktive Temperatur erhitzt und rasch abschreckt wird, ohne ihm Zeit zu lassen, den Zustand der unterkühlten Schmelze zu erreichen. Der Zustand eines Glases ist also von dessen Vergangenheit abhängig. Das „Gedächtnis“ des Glases umfasst alle vorangegangenen Heizund Kühlzyklen, zurück bis zum letzten Erreichen des metastabilen Gleichgewichts. Bei komplexeren Systemen wie Polymeren beinhaltet dieses Gedächtnis nicht nur Wärmebehandlungen, sondern auch vorangegangene Deformationen, also die Geschichte der mechanischen Beanspruchung. In diesen Fällen ist der Gleichgewichtszustand nicht nur abhängig von der Temperatur, sondern auch von den aktuell an der Probe anliegenden Kräften. So kann es zu einem Härten durch unterkritisches Dehnen (,strain hardening“) oder zu einem Erweichen des Systems durch starke Deformation (,shear softening") kommen. Eine Studie mit intensiven Auslagerungsversuchen findet sich in der Dissertation von L. C. E. Struik [STR77].

\subsection{Grundlagen der Viskoelastizität}

Die makroskopischen Eigenschaften eines amorphen Systems werden dadurch bestimmt, wie nah es dem Glasübergang ist, in Bezug auf Temperatur und Frequenz. Im Glaszustand, bei tiefen Temperaturen und hohen Frequenzen, verhält sich das System überwiegend elastisch. Im flüssigen Zustand, bei hohen Temperaturen und niedrigen Frequenzen, ist es viskos. In der Nähe des Glasübergangs wird das System als viskoelastisch bezeichnet, da es sowohl einen elastischen als auch einen viskosen Charakter besitzt. In diesem Kapitel soll nur ein Überblick der relevanten physikalischen Gesetzmäßigkeiten und Zusammenhänge gegeben werden, da es sich hierbei um ein in der Literatur und in Vorgängerarbeiten ausgiebig beschriebenes Gebiet handelt [RAM94, WEI98, RÖS94]. Eine prägnante Einführung gibt [ZAR91], empfehlenswert ist [FLS77, Bd. 2, Kap. 39ff] und eine ausführliche mathematische Beschreibung bietet [LL91].

\section{Der rein elastische Grenzfall}

Liegt an einem festen Körper eine Kraft an, so wird sich dieser deformieren. Im rein elastischen Grenzfall ist diese Deformation proportional der Spannung (Kraft/Fläche), instantan und reversibel und somit reibungsfrei. Verallgemeinert wird dies in Form eines Spannungstensors $\sigma$ (Tensor zweiter Stufe mit neun Komponenten) beschrieben, welcher über den 
Tensor der elastischen Moduln mit dem Dehnungs- oder Verzerrungstensor $\varepsilon$ verknüpft ist. Dieser Tensor der elastischen Moduln besitzt 81 Einträge, die aufgrund von Abhängigkeiten und Symmetrieüberlegungen auf 21 unabhängige Materialparameter reduziert werden können. Im einfachsten Fall wird das Material als isotrop betrachtet, d.h. dass es sich in alle Richtungen gleich verhält. In diesem Fall verringert sich die Anzahl der unabhängigen Materialparameter auf zwei [FLS77]. Beim Strecken oder Stauchen (negative Dehnung) eines Quaders wird üblicherweise der Elastizitäts- oder Youngs-Modul $E$ und die Poisson-Zahl $v$ benutzt. Aufgrund der Isotropieannahme müssen diese nicht als Tensoren beschrieben werden, sondern sind skalar.

$$
\begin{aligned}
& \sigma=E \cdot \varepsilon \\
& \sigma=\frac{F}{A} \quad \varepsilon=\frac{\Delta l}{l_{0}} \\
& v=\frac{\frac{\Delta d}{d_{0}}}{\frac{\Delta l}{l_{0}}}=\frac{\varepsilon_{\perp}}{\varepsilon} \quad 0<v \leq \frac{1}{2}
\end{aligned}
$$

Die Poisson-Zahl $v$ ist die Dickenänderung $\Delta d$ relativ zur Anfangsdicke $d_{0}$, geteilt durch die Längenänderung $\Delta l$ relativ zur Anfangslänge $l_{0}$. Dies entspricht dem Quotienten von Normaldehnung $\varepsilon_{\perp}$ und der Dehnung $\varepsilon$ in Richtung der Spannung $\sigma$. Auf die Tensor- oder Vektorschreibweise wird hier aufgrund der Einfachheit der Geometrie verzichtet, unter der Annahme, dass die Dicke senkrecht zur Länge und zur Kraft steht. Der Schermodul G und der Kompressionsmodul $K$ lassen sich dann wie folgt berechnen:

$$
G=\frac{\sigma_{t}}{\gamma}=\frac{E}{2(1+v)} \quad K=\frac{-p}{\frac{\Delta V}{V}}=\frac{E}{3(1-2 v)}
$$

Der Schermodul $G$ stellt dabei die tangentiale Spannung $\sigma_{t}$ und den Scherwinkel $\gamma$ in Radiant in Relation zu einander, während der Kompressionsmodul $K$ angibt, wie sich das Probenvolumen $V$ ändert $(\Delta V)$, wenn ein äußerer Druck $p$ ausgeübt wird.

\section{Der rein viskose Grenzfall}

Das Anlegen einer Kraft an eine Flüssigkeit ist schon deutlich schwieriger zu beschreiben, da dazu zunächst Randbedingungen eingeführt werden müssen, auf die eine Kräfte ausgeübt werden kann. Eine Deformation erfolgt dann nicht reversibel, da die geleistete Arbeit durch Reibung in Wärme umgewandelt wird. Die angelegte Kraft ist dabei proportional zur konstanten Deformationsgeschwindigkeit. Die allgemein gebräuchliche Definition der Viskosität ist daher etwas abstrakt: Wird eine Platte der Fläche $A$ parallel gegen eine zweite, gleichartige Platte im Abstand $d$ mit Flüssigkeit im Zwischenraum verschoben, so wird sich ein Geschwindigkeitsprofil zwischen den beiden ausbilden. Die Viskosität beschreibt den Zusammenhang zwischen Scherspannung $\sigma_{t}$ und Scherrate $\dot{\gamma}$ :

$$
\eta=\frac{\sigma_{t}}{\dot{\gamma}}
$$

Im uniaxialen Zugversuch ergibt sich die Viskosität analog zu Gleichung (3): 


$$
\eta=\frac{\sigma}{3 \dot{\varepsilon}}
$$

Dabei wird angenommen, dass das Volumen der Flüssigkeit gleich bleibt, während sich die Form des Flüssigkeitsbehälters ändert [ZAR91]. Die Poisson-Zahl ist somit 0,5 nach Gleichung (2). Bei Temperaturen knapp oberhalb des Glasübergangs ist die Viskosität nur bei kleinen Scher- bzw. Dehnraten unabhängig von der Scher- bzw. Dehnrate; man spricht von einem Newton'schen Bereich. Bei höheren Raten sinkt die Viskosität als Funktion der Rate; dies wird als Nicht-Newton'sches Fließen bezeichnet [LRJ03, DJ05, DHT06].

Der Verlauf der logarithmierten (Newton'schen) Viskosität in Abhängigkeit von der normierten, inversen Temperatur ist in Abbildung 2.4.1 für verschiedene Glasbildner dargestellt [ANG88, ELL90]. Dabei ist zunächst zu beachten, dass auf der Abszisse hohe Temperaturen links und tiefe rechts stehen, wobei die Glasübergangstemperatur als diejenige angenommen wird, bei der die Viskosität $10^{12}$ Pas erreicht. Somit wird ein Schnittpunkt aller Verläufe erzeugt. Die Steigung in diesem Punkt wird als Fragilität $m$ bezeichnet:

$$
m=\left.\frac{d \log \eta}{d \frac{T_{G}}{T}}\right|_{T=T_{G}}
$$

Dabei wird zwischen starken und fragilen Glasformern unterschieden, wobei festzuhalten ist, dass der Übergang von flüssig zu fest bei einem fragilen Glas in einem deutlich kleineren Temperaturfenster vonstatten geht. Bei starken Glasformern beschreibt ein Arrhenius-Gesetz den Verlauf der Viskosität, was in Abb. 2.4.1 einem linearen Verlauf entspricht:

$$
\eta=\eta_{0} \exp \left(\frac{E_{A}}{k_{B} T}\right)
$$

Der gekrümmte Verlauf der Viskosität fragiler Glasbildner wird mit einem Vogel-FulcherTammann-Gesetz beschrieben [VOG21, FUL23, TAM26]:

$$
\eta=\eta_{0} \exp \left(\frac{D \cdot T_{V F T}}{T-T_{V F T}}\right)=\eta_{0} \exp \left(\frac{B_{V F T}}{T-T_{V F T}}\right)
$$

Dabei wird $D$ als Stärkeparameter des Glasformers bezeichnet, welcher über den folgenden Zusammenhang mit der Fragilität verknüpft ist [BNA93]:

$$
m=m_{\min }+m_{\min }^{2} \cdot \frac{\ln (10)}{D} \approx 16+\frac{590}{D}
$$

Bereits an diesem wenig elementaren Zusammenhang ist abzulesen, das $T_{\mathrm{G}}$ nicht als Referenzgröße geeignet ist. Dies zeigt sich auch in Publikationen aus der aktuellen Forschung, in denen dem Schermodul (Gleichung (3)) eine zentrale Bedeutung zukommt [JS05]. 


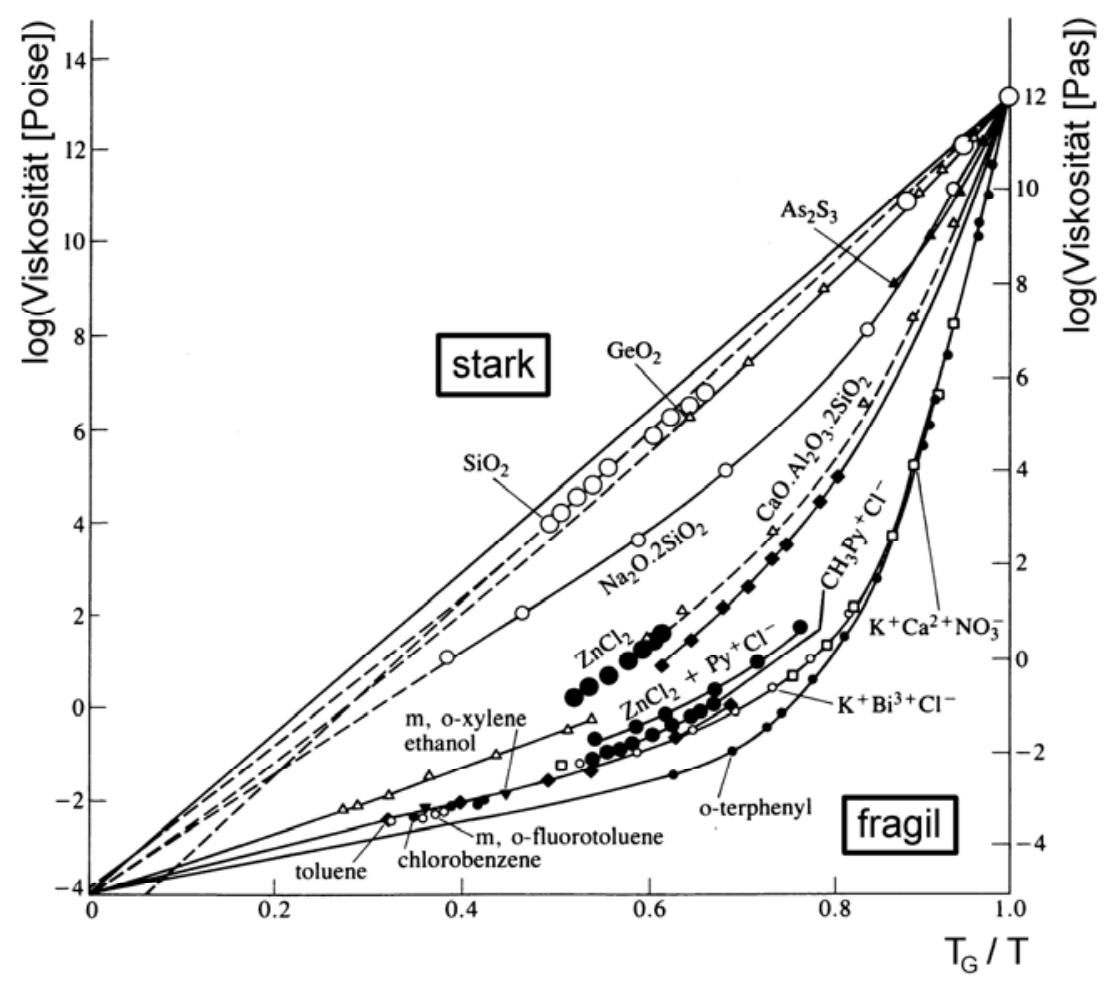

Abb. 2.4.1: Angell-Plot: Temperaturabhängigkeit der Viskosität oberhalb der Glastemperatur

\section{Mathematische Grundlagen von quasi-statischen Messungen}

Der elastische und der viskose Grenzfall gelten nur bei einer instantanen, reversiblen, beziehungsweise bei einer kontinuierlichen, irreversiblen Deformation, jedoch nicht bei endlichen Zeiten im Bereich des Glasübergangs. Bei einer instantanen Änderung der Spannung $\Delta \sigma$, kann sich die Dehnungsänderung nur so schnell einstellen, wie es die Viskosität $\eta$ des Systems zulässt. Der Übergang von der ursprünglichen Dehnung $\varepsilon_{0}$ in eine andere wird als anelastische Relaxation bezeichnet:

$$
\varepsilon_{a e}(t)=\varepsilon_{0}+\frac{\Delta \sigma}{E}\left(1-\exp \left(-\left(\frac{t}{\tau}\right)^{\beta}\right)\right) \quad \tau \propto \eta
$$

Der Streckungsparameter $\beta$ trägt dabei der Tatsache Rechnung, dass es im System nicht nur eine charakteristische Relaxationszeit gibt, sondern viele gekoppelte, die durch eine mittlere Relaxationszeit $\tau$ charakterisiert werden. Gleichung (10) beschreibt dabei nur den anelastischen Anteil der Dehnung $\varepsilon_{\mathrm{ae}}$. Hinzu kommt der rein elastische Anteil (Gleichung (2)) und der viskose Anteil $\varepsilon_{\mathrm{vis}}$ :

$$
\varepsilon_{v i s}(t)=\int_{0}^{t} \eta\left(t^{\prime}\right) d t^{\prime}
$$

Die zeitliche Einstellung einer Viskosität $\eta_{\infty}$ nach einem Sprung in der Spannung und einer ursprünglichen Viskosität $\eta_{0}$ ist noch komplizierter und wird zumeist mit einem Bimolekularfit beschrieben: 


$$
\eta_{F i t}(t)=\frac{\eta_{0}+\eta_{\infty} k \cdot\left(\frac{\eta_{\infty}-\eta_{0}}{\eta_{\infty}{ }^{2}}\right) \cdot t}{1+k \cdot\left(\frac{\eta_{\infty}-\eta_{0}}{\eta_{\infty}{ }^{2}}\right) \cdot t}
$$

Eine ausführliche Beschreibung dieser Effekte, die darstellt, wie ein viskoelastisches Material in einen neuen mechanischen Gleichgewichtszustand wechselt, findet sich in [WEI98].

\section{Dynamische Messungen an viskoelastischen Materialien}

Bei der mechanischen Spektroskopie wird eine statische Spannung $\sigma_{\text {stat }}$ an die Probe angelegt und mit einer kleineren dynamischen Spannung $\sigma^{*}$ überlagert. Dabei erweist sich das Rechnen mit komplexen Zahlen, die im Folgenden durch ein * gekennzeichnet sind, als praktisch [ZAR91]:

$$
\sigma *(t)=\sigma_{0} \exp (i \omega t)
$$

Dies führt zu einer um $\delta$ phasenverschobenen dynamischen Dehnung $\varepsilon^{*}$ :

$$
\varepsilon^{*}(t)=\varepsilon_{0} \exp (i \omega t-i \delta)
$$

$\mathrm{Da}$ in diesem Fall das System einer Spannung nachgibt, wird die Materialeigenschaft, welche die Dehnung mit der Spannung verknüpft, als Nachgiebigkeit $D^{*}$ bezeichnet:

$$
\varepsilon^{*}=D^{*} \cdot \sigma^{*}=\left(D^{\prime}-i D^{\prime \prime}\right) \cdot \sigma^{*} \quad \frac{D^{\prime}}{D^{\prime \prime}}=\tan \delta
$$

Es ergibt sich hier ein konzeptioneller Unterschied, wenn im umgekehrten Fall dem System eine oszillierende Dehnung aufgezwungen, und die Spannung beobachtet wird. Dann gilt:

$$
\sigma^{*}=E^{*} \cdot \varepsilon^{*}=\left(E^{\prime}+i E^{\prime \prime}\right) \cdot \varepsilon^{*} \quad \frac{E^{\prime \prime}}{E^{\prime}}=\tan \delta
$$

Der Materialparameter ist in diesem Fall der Elastizitäts- oder Youngs-Modul $E^{*}$. Im Fall des eingeschwungenen Oszillators gilt [ZAR91]:

$$
E^{*}=\frac{1}{D^{*}}
$$

Es wird von daher im Folgenden mit dem Elastizitätsmodul $E^{*}$ gearbeitet. Dieser ist jedoch keineswegs konstant, sondern bei viskoelastischen Materialien, wie zum Beispiel bei amorphen Systemen in der Nähe des Glasübergangs, stark frequenz- und temperaturabhängig. Wird dabei die Frequenz $f$ der erzwungenen Schwingung resonant mit der Relaxationszeit $\tau$ des Materials, so ist der (Glas-)Übergang im Material als Änderung im Realteil $E$ ' und als Maximum im Imaginärteil $E$ ' zu beobachten. Eine genauere Beschreibung des Übergangs als die Deborahzahl $D E$ (vgl. Kap. 2.1) bietet also die Resonanzbedingung:

$$
1=\omega \cdot \tau=2 \pi f \cdot \tau
$$

Die Form des Elastizitätsmoduls lässt sich im Falle nur einer Relaxationszeit $\tau$ mit einer Debye-Funktion beschreiben [ZAR91]:

$$
E^{*}(\omega)=E_{\infty}-\frac{\Delta E}{1+i \omega \tau} \quad \Delta E=E_{\infty}-E_{0}
$$


$E_{0}$ ist dabei der Realteil des Elastizitätsmoduls bei der Frequenz $f=0$, während $E_{\infty}$ dem Realteil des Elastizitätsmoduls bei unendlich hohen Frequenzen entspricht. Da zumeist aber mehrere gekoppelte Relaxationszeiten vorliegen, gibt es eine Vielzahl von Modifikationen, mit denen Gleichung (19) an eine Messung angepasst werden kann [SVA03]. Als sehr allgemein einsetzbar zeigt sich dabei die Funktion nach Havriliak und Negami [HN67]:

$$
E^{*}(\omega)=E_{\infty}-\frac{\Delta E}{\left(1+(i \omega \tau)^{\alpha}\right)^{\gamma}}
$$

Wird $0<\alpha<1$ und $\gamma=1$ gewählt, so entsteht eine symmetrische Verbreiterung, was einer Cole-Cole-Funktion entspricht [CC41]. $\alpha=1$ und $0<\gamma<1$ führt zu einer Asymmetrie, auch als Cole-Davidson-Funktion bezeichnet [DC50, DC51]. Das Ersetzen von $\tau$ durch die zu Gleichung (8) analoge Vogel-Fulcher-Tammann-Funktion $(\eta \sim \tau)$, bei Messung mit konstanter Frequenz $f$, ergibt eine Parametrisierung des Elastizitätsmoduls als Funktion der Temperatur [RÖS04]:

$$
E *(T)=E_{\infty}-\frac{\Delta E}{\left(1+\left(i 2 \pi f \tau_{0} \exp \left(\frac{B_{V F T}}{T-T_{V F T}}\right)\right)^{\alpha}\right)^{\gamma}}
$$

Die Fitfunktionen folgen aus der Zerlegung in Real- und Imaginärteil, auch bezeichnet als Speicher- und Verlustmodul des Elastizitätsmoduls [RÖS04]:

$$
\begin{gathered}
E^{\prime}(T)=E_{\infty}-\frac{\Delta E \cos (\gamma \varphi)}{\left(1+2 \cdot\left(2 \pi f \tau_{V F T}\right)^{\alpha} \cdot \cos \left(\alpha \frac{\pi}{2}\right)+\left(2 \pi f \tau_{V F T}\right)^{2 \alpha}\right)^{\frac{\gamma}{2}}} \\
E^{\prime \prime}(T)=\frac{\Delta E \sin (\gamma \varphi)}{\left(1+2 \cdot\left(2 \pi f \tau_{V F T}\right)^{\alpha} \cdot \cos \left(\alpha \frac{\pi}{2}\right)+\left(2 \pi f \tau_{V F T}\right)^{2 \alpha}\right)^{\frac{\gamma}{2}}} \\
\varphi:=\arctan \left(\frac{\left(2 \pi f \tau_{V F T}\right)^{\alpha} \cdot \sin \left(\alpha \frac{\pi}{2}\right)}{1+\left(2 \pi f \tau_{V F T}\right)^{\alpha} \cdot \cos \left(\alpha \frac{\pi}{2}\right)}\right) \tau_{V F T}=\tau_{0} \exp \left(\frac{B_{V F T}}{T-T_{V F T}}\right)=\tau_{0} \exp \left(\frac{D_{V F T} \cdot T_{V F T}}{T-T_{V F T}}\right)
\end{gathered}
$$

\subsection{Relaxationen im Glaszustand}

In diesem Kapitel soll ein Überblick auf das Relaxationsspektrum eines amorphen Materials gegeben werden. Dies zeigt gleichzeitig die Vorgänge, die prinzipiell spektroskopisch gemessen werden können.

Abbildung 2.5.1 zeigt ein charakteristisches Relaxationsspektrum eines amorphen Systems [LL00] in Form einer schematischen Messung des dielektrischen Verlusts bei zwei Temperaturen als Funktion der Frequenz, die dabei 21 Dekaden überspannt. Die einzelnen Relaxationen sind dabei unterschiedlich eingefärbt. Da die dynamischen Prozesse auf einander aufbauen, ist es sinnvoll, das Spektrum von rechts nach links zu beschreiben: 


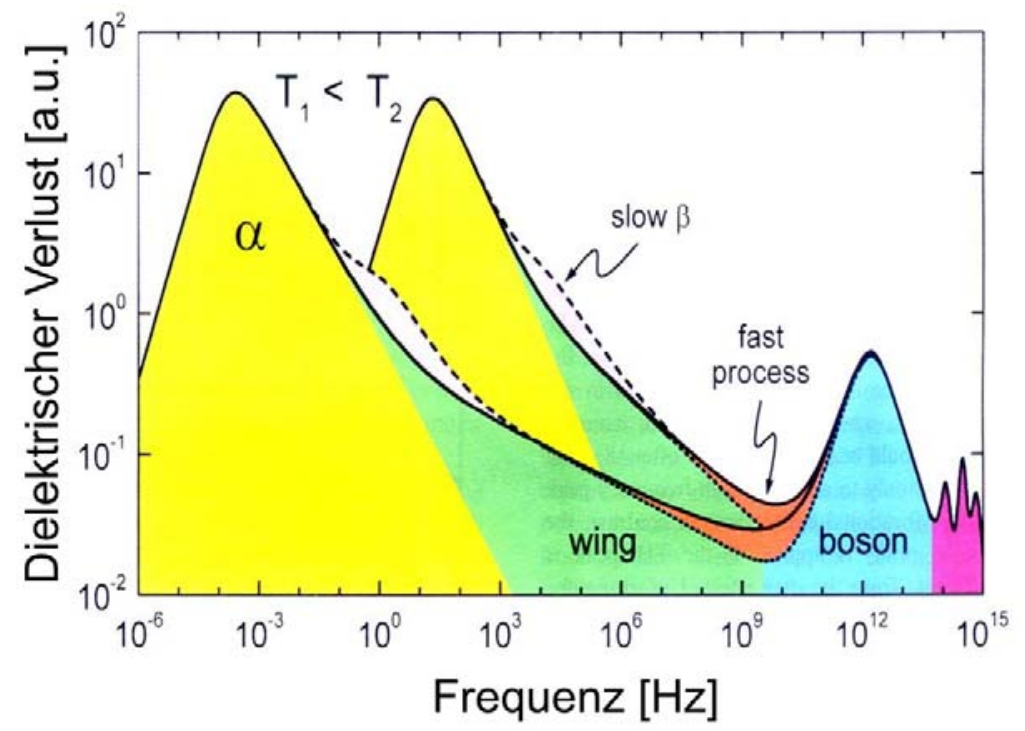

Abb. 2.5.1: Lunkenheimer-Loidl-Plot: Schematische Darstellung des Relaxationsspektrums

Oberhalb $10^{14} \mathrm{~Hz}$ wird die Dynamik bestimmt durch Molekülschwingungen im Infrarotbereich [IRS95]. Unterhalb, bis etwa $10^{10} \mathrm{~Hz}$, wird sie durch "Gitter"-Schwingungen dominiert. Diese kooperative Bewegung führt zum so genannten "Boson-Peak" im Verlustspektrum [CSB04, ANG04]. Darunter ist ein Minimum zu beobachten, gefolgt von einem nahezu konstanten Bereich, dem "Nearly Constant Loss" (NCL). Diese beiden können im Rahmen der Modenkopplungstheorie erklärt werden [GÖT98]. Der in diesem Bereich vorherrschende "Fast Process", auch als schnelle $\beta$-Relaxation bezeichnet, wird zumeist mit dem so genannten "Cage-Ratteling" in Verbindung gebracht, bei dem ein Teilchen sich im festen Käfig aus anderen Teilchen bewegt, ohne diesen verlassen zu können. Bei noch tieferen Frequenzen wird der Bereich des "Wing" erreicht, wobei nicht klar ist, ob es sich um eine Flanke oder ein Maximum handelt, das von der angrenzenden $\alpha$-Relaxation überlagert wird [DYR05]. Bei einigen Glasformern wird in diesem Bereich dieses Maximum beobachtet, die so genannte langsame $\beta$-Relaxation [KBB99]. Wie stark $\alpha$ - und $\beta$-Relaxation einander überdecken ("Merging“), hängt dabei stark von der Temperatur bzw. Frequenz ab [EAN96, DON01]. Die beiden Prozesse sind bei tiefen Temperaturen bzw. niedrigen Frequenzen besser getrennt. Der Versuch des grundsätzlichen Nachweises der Existenz einer langsamen $\beta$-Relaxation wurde in [RÖS04, RSL04] begonnen. Im Bereich der $\beta$-Relaxation konnte in Simulationen gezeigt werden, dass zwar kooperative Prozesse vorliegen, diese jedoch nicht zu einer plastischen Deformation des Materials führen können [TEI05, VLZ05]. Die $\alpha$-Relaxation stellt schließlich den Übergang ins viskose Fließen dar, wobei die Probe makroskopisch deformiert wird.

Leider ist festzuhalten, dass es noch keine umfassende Theorie gibt, die in der Lage wäre, $\alpha$ und $\beta$-Relaxationen und den NCL miteinander zu verbinden. Erschwert wird die Situation zusätzlich dadurch, dass es aufgrund divergierender Zeitskalen nicht möglich ist, im Gleichgewicht zu messen, wodurch zusätzlich Alterungseffekte des Materials beobachtet werden. Dabei verhalten sich $\alpha$ - und $\beta$-Relaxation unterschiedlich. Das Maximum der $\alpha$-Relaxation verschiebt sich beim Auslagern schneller zu kleineren Frequenzen als $\beta$, dessen Intensität dabei absinkt [LWU05]. 


\subsection{Polymerdynamik}

Komplexer wird die Situation im Polymer dadurch, dass die Annahme, es mit einem System aus harten Kugeln zu tun zu haben, nicht mehr gerechtfertigt ist. Polymere sind lange, kettenförmige Makromoleküle, die aus den chemisch verbundenen Monomereinheiten aufgebaut sind. Je nachdem, ob es sich nur um eine Monomersorte oder mehrere handelt, wird von einem Homo- oder Copolymer gesprochen, wobei auch die Verteilung der Monomere entlang des Polymers entscheidend ist. Die chemischen Eigenschaften der Monomereinheiten können dabei sehr verschieden sein in Hinblick auf Wechselwirkungen, Beweglichkeit und auf die Möglichkeit Verzweigungen oder Vernetzungen mit anderen Polymeren bilden zu können. Diese Vielfalt in der Mikrostruktur spiegelt sich in den makroskopischen mechanischen Eigenschaften und dem Relaxationsspektrum wieder, da im Prinzip jede Struktureinheit zu weiteren, charakteristischen Relaxationen führen kann.

Obwohl die Relaxationen stark von den chemischen Eigenschaften abhängen, gibt es doch für Polymere charakteristische Relaxationen, auf die im Folgenden kurz eingegangen werden soll [RIC02, SPE92, WAR83, DE88, MRW67, MEN99].

\section{Kurbelwellen- und Seitengruppenbewegungen}

Bei der Kurbelwellenbewegung nach Boyer und Schatzki [MRW67, SPE92] wird von einer linearen Kette mit festen Winkeln zwischen den Bindungen ausgegangen, wobei aber um eine Bindung frei gedreht werden kann. Boyer schlägt dabei vor, sechs $\mathrm{CH}_{2}$-Einheiten eines Polyethylenmoleküls zu betrachten (Abb. 2.6.1 b)). Diese sind über fünf Bindungen aufgereiht, wobei die Bindungen eins und fünf kolinear festgehalten werden und die Bindungen zwei bis vier um die Bindungen eins und fünf gedreht werden. Schatzki schlägt vor, acht $\mathrm{CH}_{2}$ Einheiten mit sieben Bindungen dazwischen zu betrachten (Abb. 2.6.1 a)). Die Rotation der S-förmigen Gruppe der Atome zwei bis sieben um die kolinearen Bindungen eins und sieben gibt der Kurbelwellenbewegung ihren Namen. Bei einer Frequenz von $1 \mathrm{~Hz}$ liegt diese Relaxationsmode bei ca. 153 K [MRW67]. Auch Seitengruppen haben ihre charakteristischen Bewegungsmoden. Diese liegen im Bereich der Kurbelwellenbewegung oder darüber, je nachdem, wie groß die relaxierende Seitengruppe ist. Bei Polystyrol beispielsweise ist eine Torsion der Phenylseitengruppe bei $325 \mathrm{~K}$ [SPE92] zu beobachten. Je nach Nomenklatur werden Kurbelwellenrelaxationen zumeist als $\gamma$ - und Seitenkettenrelaxationen als $\beta$-Relaxation bezeichnet, nicht zu verwechseln mit der schnellen oder der langsamen $\beta$-Relaxation aus dem vorangegangenen Unterkapitel 2.5.

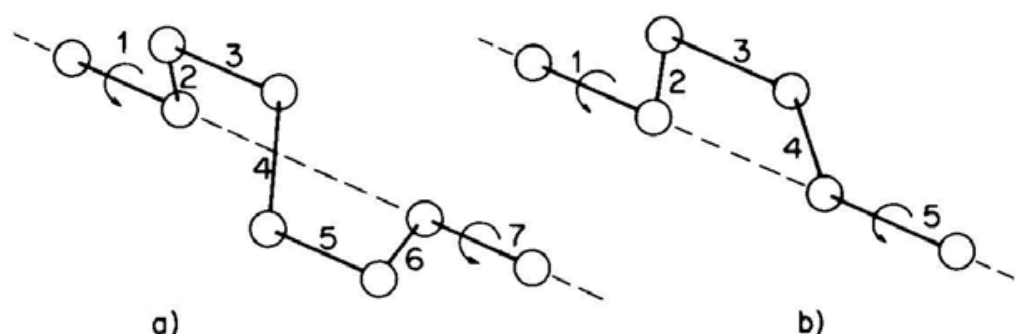

Abb. 2.6.1: Kurbelwellenbewegung nach a) Schatzki und b) Boyer 


\section{Segmentbewegungen nach Rouse}

Im klassischen harmonischen Oszillator wird ein Wechselspiel aus Trägheitskraft und Federkraft betrachtet. Die Rückstellkraft im Rouse-Modell ist von entropischer Natur, weshalb auch von der entropischen Feder gesprochen wird. Dies hat zur Folge, dass ein Polymer immer den geknäulten Zustand bevorzugt, da es für diesen mehr Realisierungsmöglichkeiten gibt als für ein gestrecktes Polymer. Der Ansatz lautet [BK05, DE88, RIC02]:

$$
\underbrace{m \frac{\vec{d}^{2} \vec{x}_{j}}{d t^{2}}}_{\text {Trägheit. }}=\underbrace{\vec{F}_{S}}_{\text {Entropie }}+\underbrace{\vec{F}_{R}}_{\text {Reibung }}+\underbrace{\vec{F}_{F l}}_{\text {Fluktuation }}
$$

Die Gleichung (24) wird gelöst durch Fourier-Transformation. Für die Relaxationszeiten im Rouse-Spektrum folgt:

$$
\tau_{p}=\frac{n^{2} a^{2} \varsigma}{3 k_{B} T \pi^{2} p^{2}} \quad p=1 ; 2 ; 3 ; \ldots
$$

Dabei ist $n$ die Anzahl der frei drehbaren Kuhn-Segmente [DE88] der Länge $a, \zeta$ der Reibungskoeffizient und $p$ die Modenzahl.

Das Rouse-Modell findet für Polymerschmelzen Anwendung und kann als wesentlicher Prozess des Glasübergangs ( $\alpha$-Relaxation) betrachtet werden. Es schwingen ganze Kettensegmente. Die Kettenlänge der Polymere beeinflusst also den Glasübergang, wenn sie im Bereich der Segmentgröße liegt. Oberhalb einer Kettenlänge von einigen Hundert Monomereinheiten ist die Glasübergangstemperatur konstant, die typische Größe eines schwingenden Segments sollte also darunter liegen [SPE92].

\section{Abgleiten von ganzen Ketten im Reptationsmodell}

Oberhalb der Glastemperatur, die in der Chemie auch Erweichungspunkt genannt wird, sind langkettige Polymere auf endlichen Zeitskalen noch nicht flüssig, sondern verhalten sich Gummi-elastisch. Gummi, auch Elastomer genannt, entsteht durch die Erzeugung von Vernetzungspunkten, die ein Abgleiten des ganzen Polymers verhindern. Dasselbe Verhalten bei langkettigen, unvernetzten Polymeren wird dadurch erklärt, dass die Polymerketten temporäre Verhakungspunkte besitzen, die ein viskoses Fließen bis zur Übergangstemperatur in die Flüssigkeit verhindern [SPE92]. Dieser Übergang wird als $\alpha^{\prime}$-Relaxation, Flüssig-FlüssigÜbergang oder auch irreführend als Schmelztemperatur (Kristalle schmelzen, Gläser nicht) bezeichnet [MRW67, SPE92, MEN99] und hängt stark von der Kettenlänge ab [DE88].

Zwischen dieser Temperatur und dem Glasübergang ( $\alpha$-Relaxation) wird die Dynamik der Polymerkette durch temporäre Verhakungspunkte begrenzt, deren Abstände in der Größenordnung von 3 bis $10 \mathrm{~nm}$ liegen [RIC02]. Das Polymer kann sich nur im Inneren einer Röhre bewegen. Eine Bewegung entlang der Röhre ist nur möglich, indem es sich hindurchschlängelt. Diese Relaxationsmode, die auf DeGennes zurückgeht, wird als Reptation bezeichnet [RIC02, SPE92]. Die wesentlichen Aussagen dieses Modells sind, dass sich die Relaxationszeit proportional zur dritten Potenz der Kettenlänge verhält, während der Realteil des Schermoduls in diesem Temperaturbereich ein Plateau aufweist, dessen Höhe proportional zur Kettenlänge ist. 


\section{Materialien und Methoden}

Ziel dieses Kapitels ist die Beschreibung der verwendeten Materialien und Methoden. Zunächst wird die Probenherstellung geschildert, anschließend die Probencharakterisierung einführend beschrieben und abschließend werden die spektroskopischen Untersuchungsmethoden, insbesondere die dynamisch-mechanische Analyse (DMA), erläutert.

\subsection{Präparation von Bändern aus metallischem Glas}

\section{Herstellung einer Vorlegierung im Lichtbogenofen}

Der erste Schritt bei der Herstellung von metallischen Gläsern ist zumeist die Herstellung einer Vorlegierung aus den elementaren Reinmaterialien, wie in Tabelle 3.1.1 aufgelistet. Sinnvoll ist dabei, mit Materialstücken in gut handhabbaren Größen zu arbeiten. Insbesondere ist bei Materialien, die mit Luft reagieren können, darauf zu achten, dass diese nicht als Pulver vorliegen. Bei Zirkonium muss zunächst die Oberfläche abgefeilt werden, um es von seiner Oxidschicht zu befreien. Kontaminationen des Materials können als Kristallisationskeime fungieren, womit die Präparation einer amorphen Probe erschwert und auch die Qualität des Materials bei den späteren Versuchen verschlechtert wird. Anschließend werden die Komponenten der Legierungen abgewogen, wobei die Zusammensetzungen in Atomprozent $\mathrm{Pd}_{77,5} \mathrm{Cu}_{6} \mathrm{Si}_{16,5}$ und $\mathrm{Zr}_{65} \mathrm{Al}_{7,5} \mathrm{Cu}_{27,5}$ sind. Das Gesamtgewicht der Probe sollte für eine gute Handhabbarkeit etwa 3,5 bis $4 \mathrm{~g}$ betragen.

Tab. 3.1.1: Tabelle der verwendeten Materialien

\begin{tabular}{|l|l|l|l|}
\hline Element & Hersteller & Form & Reinheit \\
\hline Aluminium & Alfa Aesar & Stangen & $4 \mathrm{~N} 8$ \\
\hline Kupfer & Strem Chemicals & Granulat & $5 \mathrm{~N}$ \\
\hline Palladium & Goodfellow & Granulat & $3 \mathrm{~N} 5$ \\
\hline Silizium & Alfa Aesar & Block & $6 \mathrm{~N}$ \\
\hline Zirkonium & Wah Chang & Block & $5 \mathrm{~N}$ \\
\hline
\end{tabular}

Die Herstellung der Vorlegierung erfolgt dann in einem Lichtbogenofen Typ MAM-1 der Firma Bühler. Nach Positionierung der Probenstücke in einer Mulde der wassergekühlten Kupferplatte, wird fünfmal evakuiert und mit Argon der Reinheit 5N gespült. Bei einem Arbeitsdruck von 400 mbar wird zunächst ein Zirkonium-Getterblock aufgeschmolzen, der den verbliebenen Sauerstoff bindet. Anschließend wird das Material durch Zusammenschmelzen legiert und mehrfach gedreht und neu aufgeschmolzen, um eine Homogenisierung des Materials zu erreichen.

\section{Melt Spinning}

Beim so genannten Melt Spinning wird eine Schmelze auf ein rotierendes Kupferrad gespritzt. Dabei erstarrt diese und wird in Form eines langen Bandes tangential weggeschleudert. Die Kühlrate liegt dabei im Bereich $10^{5} \mathrm{~K} / \mathrm{s}$ [JON81]. Die prinzipielle Funktionsweise zeigt Abbildung 3.1.1. 


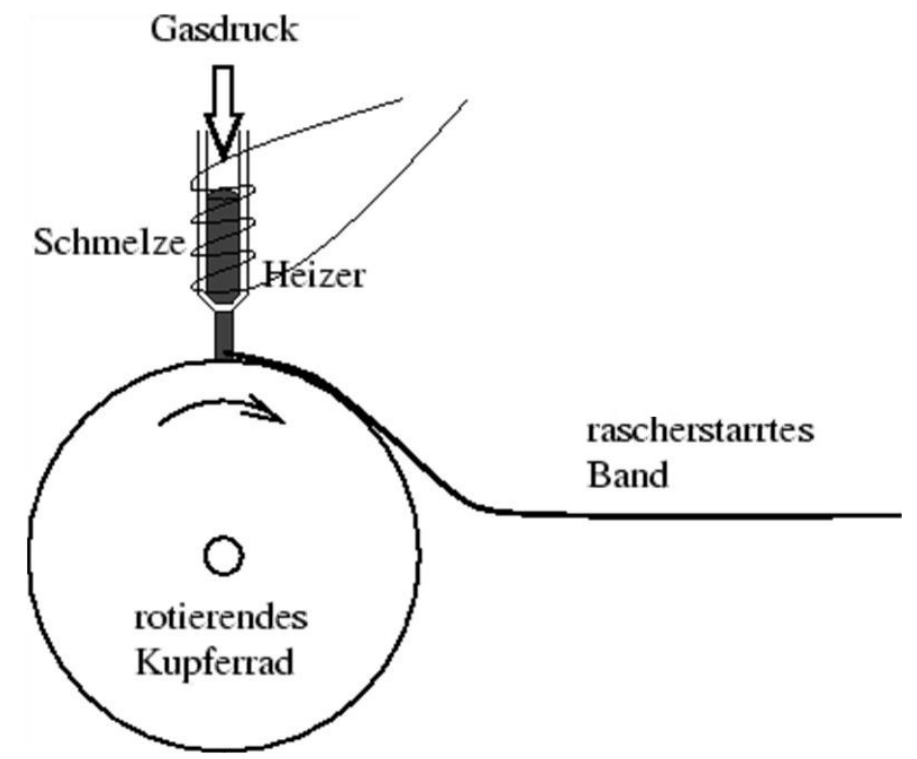

Abb. 3.1.1: Funktionsweise des Melt Spinners [IRÖ04]

Bei der Vorbereitung eines Schusses wird zunächst die zerstoßene Vorlegierung in ein Quarzrohr mit einem Düsen-Durchmesser von ca. $1 \mathrm{~mm}$ gefüllt und dieses mit festem Abstand über dem polierten Kupferrad in der Vakuumkammer justiert. Die für die Proben verwendeten Parameter enthält Tabelle 3.1.2. Es handelt sich dabei um empirisch gewonnene Parameter, die zu einer amorphen Probe geführt haben. Ist der Abstand zu klein, ist die Kühlrate reduziert und es wird zusätzlich eine Beschädigung der Anlage riskiert, wenn die Schmelze erstarrt, während sie noch in Kontakt mit dem Quarzrohr ist. Bei einem zu großem Abstand fasert das Material auf. Nach fünfmaligem Evakuieren und Spülen mit Argon der Reinheit 5N werden der Arbeitsdruck im Rezipienten und der Ausblasdruck eingestellt und das Rad in Rotation versetzt. Die Probe wird dann induktiv aufgeschmolzen und auf das wassergekühlte Kupferrad geblasen, wenn die Schmelze droht, von allein aus der Düse zu fließen. Dabei ist es wichtig, dass die Probe vollständig geschmolzen und hinreichend dünnflüssig ist, da sonst die Probe inhomogen sein kann, das Ausblasen nicht funktioniert oder Düse, Rad und Heizer beschädigt werden können. Das Aufschmelzen sollte aber auch nicht länger und bei höheren Temperaturen als nötig erfolgen, da sonst eine Reaktion mit dem Quarzrohr nicht mehr ausgeschlossen werden kann, was zu einer Verfälschung der Probenzusammensetzung führt.

Tab. 3.1.2: Tabelle der Herstellungsparameter beim Melt Spinning

\begin{tabular}{|l|l|l|l|l|l|l|}
\hline Probe & $\begin{array}{l}\text { Durch- } \\
\text { messer }\end{array}$ & Abstand & $\begin{array}{l}\text { Rotations- } \\
\text { frequenz }\end{array}$ & $\begin{array}{l}\text { Druck } \\
\text { Rezipient }\end{array}$ & $\begin{array}{l}\text { Ausblas- } \\
\text { druck }\end{array}$ & $\begin{array}{l}\text { Leistung } \\
\text { [a. u. }\end{array}$ \\
\hline $\mathrm{Pd}_{77,5} \mathrm{Cu}_{6} \mathrm{Si}_{16,5}$ & $0,8 \mathrm{~mm}$ & $0,3 \mathrm{~mm}$ & $60 \mathrm{~Hz}$ & 0,5 bar & $0,8 \mathrm{bar}$ & 8,9 \\
\hline $\mathrm{Zr}_{65} \mathrm{Al}_{7,5} \mathrm{Cu}_{27,5}$ & $1,1 \mathrm{~mm}$ & $0,3 \mathrm{~mm}$ & $60 \mathrm{~Hz}$ & $0,5 \mathrm{bar}$ & $0,8 \mathrm{bar}$ & 10,0 \\
\hline
\end{tabular}

Das eigentliche Abschrecken erfolgt beim Kontakt mit dem Kupferrad, das Band ist aber auch danach noch heiß und kühlt im Flug weiter ab. Deshalb ist es sinnvoll, einen relativ hohen Druck im Rezipienten zu wählen und beide Flugrohre zu benutzen, da sonst das Band beim Aufschlag auf den Rohrdeckel stark verformt wird. Eine Verbesserung der Kühlrate kann man auch durch ein schnelleres Rotieren erreichen. Dies geschieht aber auf Kosten der Probendi- 
cke. Zusammenfassend begünstigen hohe Rotationsgeschwindigkeiten und Drücke knapp unterhalb 1 bar die Raschabschreckung, während der Abstand zwischen Düse und Rad nicht zu groß gewählt werden sollte, um ein über mehrere Meter gleichmäßiges Band von etwa $30 \mu \mathrm{m}$ Dicke und $1 \mathrm{~mm}-2 \mathrm{~mm}$ Breite zu erzeugen.

\subsection{Radikalische Hochdruck-Copolymerisation von Poly(Ethylen-co- Methacrylsäure)}

Zur Herstellung des Copolymers Poly(Ethylen-co-Methacrylsäure) (EMAA) wird der in der Abteilung von Prof. Dr. M. Buback betriebene kontinuierliche Hochdruck-HochtemperaturRührkessel verwendet. Die chemischen und apparativen Grundlagen sind in vorangegangenen Dissertationen [z.B. WITT98, BOX00, PAN92] ausführlich beschrieben und sollen hier nur knapp verdeutlicht werden.

Bei der radikalischen Copolymerisation von Ethen und Methacrylsäure (MAA) handelt es sich in sofern um eine besondere Reaktion, als dass die Homopolymere aus nur einer Monomersorte unter Normalbedingungen nicht mischbar sind. Der Grund dafür liegt in der chemischen Struktur, wie in Abbildung 3.2.1 gezeigt. Eingebaut im Copolymer können die unpolaren Ethylen-Einheiten nur über Van-der-Waals-Wechselwirkungen einander anziehen, während die polaren MAA-Gruppen untereinander Wasserstoffbrückenbindungen bilden können.

a)

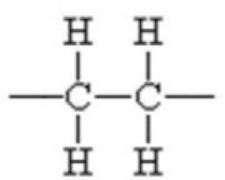

b)

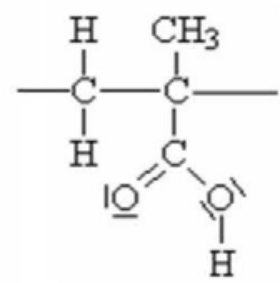

Abb. 3.2.1: Chemische Struktur der Monomereinheiten, eingebaut im Copolymer: a) Ethylen, b) Methacrylsäure

Um ein homogenes Copolymer zu erhalten, ist jedoch während der Reaktion eine homogene Mischung der Monomere unabdingbar. Deshalb wird der Reaktor bei hohem Druck und erhöhter Temperatur betrieben, da die Methacrylsäure in Ethen im superkritischen Zustand gelöst werden kann. Zusätzlich bietet die Methode des kontinuierlich betriebenen Rührkessels weitere Vorteile. Da ständig ein konstanter Zufluss von Monomeren und Initiatorlösung und ein konstanter Ausfluss von in Ethen gelöstem Copolymer durch die Reaktionskammer aufrechterhalten wird, findet die Reaktion bei guter Durchmischung unter stationären Bedingungen bezüglich der Konzentrationen, des Drucks und der Temperatur statt. Dies garantiert eine gleich bleibend hohe chemische Einheitlichkeit des produzierten Copolymers. Der apparative Aufwand, um dies zu ermöglichen, ist erheblich, wie in Abbildung 3.2.2 dargestellt. Das Ethen wird mittels eines Kupfer-Katalysators von Sauerstoffverunreinigungen befreit, durch ein Molsieb getrocknet und anschließend in einem zweistufigen Kompressor auf 260 - 280 bar vorkomprimiert. Die Ethen-Fördermenge liegt bei $1,8 \mathrm{~kg} / \mathrm{h}$. Dann wird unverdünnte Methacrylsäure zugesetzt, wobei der Fluss bei ca. $5-100 \mathrm{ml} / \mathrm{h}$ liegt. Die Zugabe der Initiatorlösung aus in Cyclohexan gelöstem Trigonox F-C50 (TxF) und Trigonox B (TxB) erfolgt 
durch eine Pumpe, wie sie auch zur Hochleistungs-Flüssigkeits-Chromatographie (HPLC) verwendet wird.

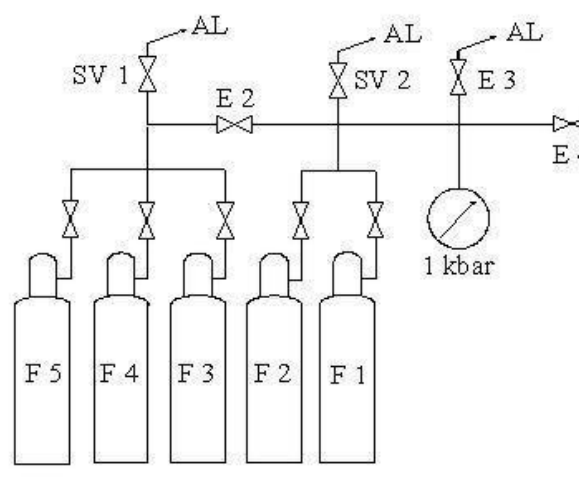

Ethen-Vorrat

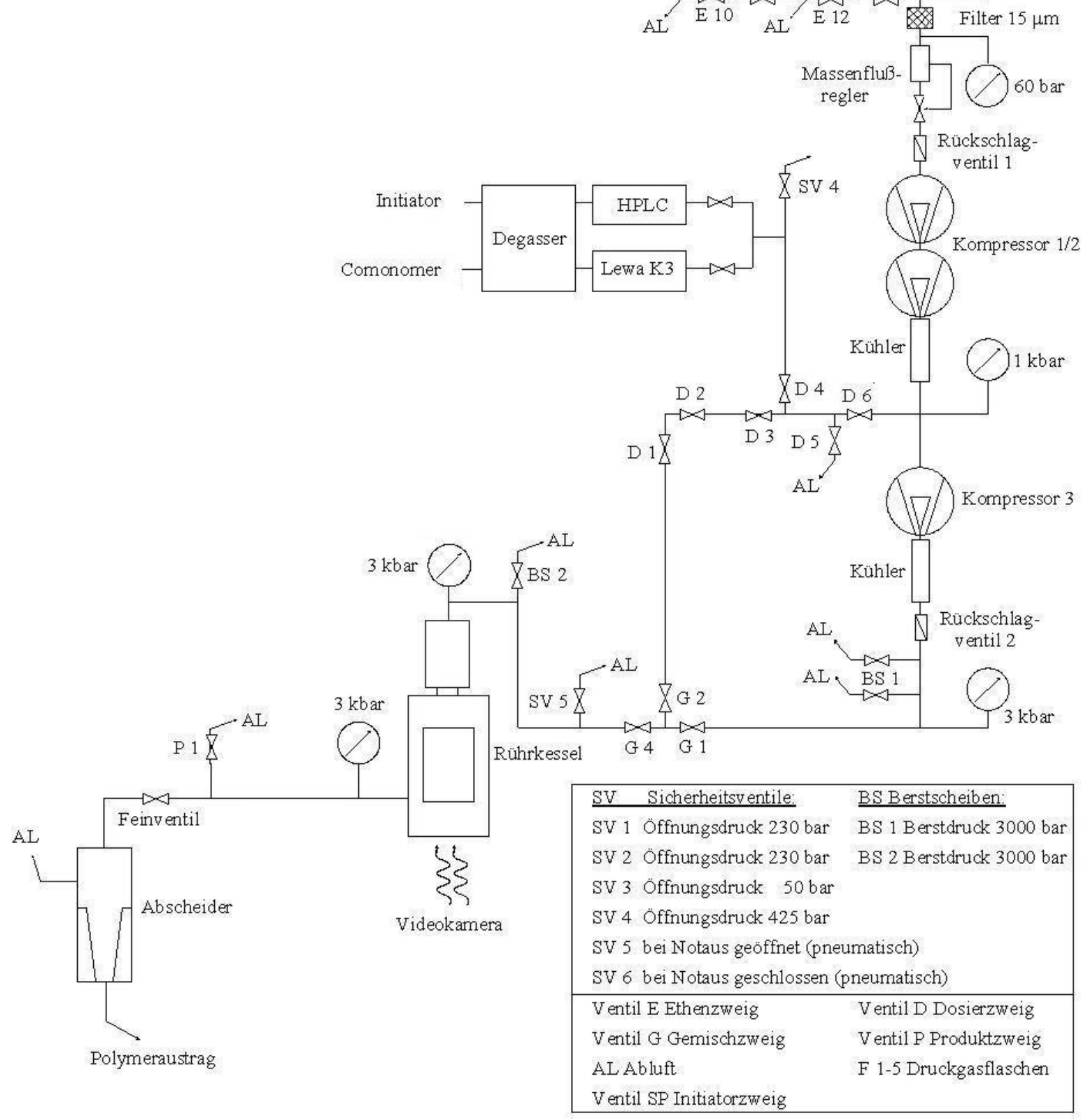

Abb. 3.2.2: Technisches Schaltbild des kontinuierlich arbeitenden HochdruckHochtemperatur-Copolymerisations-Reaktors 
Tab. 3.2.1: Tabelle der Polymerisationsparameter

\begin{tabular}{|l|l|l|l|l|l|l|}
\hline $\begin{array}{l}\text { Proben- } \\
\text { bezeich- } \\
\text { nung }\end{array}$ & $\begin{array}{l}\text { MAA- } \\
\text { Fluss } \\
{[\mathrm{g} / \mathrm{h}]}\end{array}$ & $\begin{array}{l}\text { Fluss } \\
\text { Initiator- } \\
\text { Lösung } \\
{[\mathrm{ml} / \mathrm{h}]}\end{array}$ & $\begin{array}{l}\text { Initiator- } \\
\text { konzen- } \\
\text { tration } \\
{[\mathrm{mmol} / \mathrm{l}]}\end{array}$ & $\begin{array}{l}\text { Synthese- } \\
\text { druck } \\
{[\mathrm{bar}]}\end{array}$ & $\begin{array}{l}\text { Mantel- } \\
\text { tempera- } \\
\text { tur }[\mathrm{K}]\end{array}$ & $\begin{array}{l}\text { Innen- } \\
\text { tempera- } \\
\text { tur }[\mathrm{K}]\end{array}$ \\
\hline $\begin{array}{l}\text { EMAA } \\
1.2\end{array}$ & 34,2 & 23,1 & $\begin{array}{l}\text { TxF: 23,2 } \\
\text { TxB: } 6,93\end{array}$ & 2000 & 515 & 528 \\
\hline $\begin{array}{l}\text { EMAA } \\
2.2\end{array}$ & 34,2 & 27,2 & $\begin{array}{l}\text { TxF: 23,4 } \\
\text { TxB: } 7,03\end{array}$ & 1860 & 518 & $544-549$ \\
\hline $\begin{array}{l}\text { EMAA } \\
2.10\end{array}$ & 34,2 & 57,9 & $\begin{array}{l}\text { TxF: } 23,4 \\
\text { TxB: } 7,03\end{array}$ & 1300 & 519 & $541-545$ \\
\hline $\begin{array}{l}\text { EMAA } \\
3.4\end{array}$ & 116,0 & 94,0 & $\begin{array}{l}\text { TxF: } 23,2 \\
\text { TxB: } 6,91\end{array}$ & 2300 & 517 & 545 \\
\hline $\begin{array}{l}\text { EMAA } \\
3.12\end{array}$ & 116,0 & 455,0 & $\begin{array}{l}\text { TxF: } 23,2 \\
\text { TxB: } 6,91\end{array}$ & 1300 & 518 & $541-544$ \\
\hline $\begin{array}{l}\text { EMAA } \\
12.4\end{array}$ & 5,3 & 9,9 & TxF: 46,5 & 1300 & 518 & $536-537$ \\
\hline $\begin{array}{l}\text { EMAA } \\
12.11\end{array}$ & 5,3 & 2,4 & TxF: 46,5: & 2300 & 519 & $541-548$ \\
\hline
\end{tabular}

*: Angegeben ist die Konzentration in der zugesetzten Initiator-Lösung

Die noch heterogene Mischung wird dann in der dritten Kompressorstufe auf den Arbeitsdruck von 1300 - 2300 bar komprimiert und in den Rührkessel geleitet. Dessen Manteltemperatur liegt bei etwa $518 \mathrm{~K}$, die Temperatur im Inneren des Reaktors ist noch zusätzlich durch die exotherme Reaktion erhöht. Die genauen Prozessparameter zeigt Tabelle 3.2.1. Die Homogenität während der Polymerisation wird mittels einer Videokamera durch ein Saphirfenster im Boden des Reaktors überwacht. Die Steuerung des Drucks erfolgt durch ein Feinventil vor dem Abscheider, wo das verbliebene Ethen entweicht und das Copolymer ausfällt und aufgefangen wird. Jeder der Parameter, Konzentration der Methacrylsäure und des Initiators im superkritischen Ethen, Druck und Reaktionstemperatur, beeinflusst maßgeblich die Polymerisationsreaktion. Diese sind aber nicht unabhängig von einander wählbar, wenn der Reaktor in einem stationären Zustand betrieben werden soll. Der Methacrylsäure- und der Initiatorfluss werden zunächst fest eingestellt. Dann gilt es mit den Parametern Druck und Leistung des Mantelheizers einen stabilen Zustand zu erreichen. Die Polymerisationsreaktion beginnt bei ausreichend hohem Druck und hohen Temperaturen. Da sie exotherm verläuft, muss sofort die Leistung reduziert und der Druck zunächst wieder gesenkt werden, da der Heizer träge ist. Steigt die Temperatur zu stark an, beschleunigt sich die Reaktion weiter, was im schlimmsten Fall dazu führt, dass sich das Polymer zersetzt und die Anlage verstopft. Bei zu starkem Gegensteuern wird die Reaktion gelöscht und muss neu gestartet werden. Eine ausreichende Initiatorkonzentration kann diese Empfindlichkeit abschwächen, führt aber auch zu einer ungewünschten Verkürzung der Kettenlänge des Copolymers. Die zur Initiierung einer 
Polymerisationsreaktion optimale Temperatur liegt bei TxF bei $473 \mathrm{~K}$ und bei TxB bei $513 \mathrm{~K}$. Ein höherer Anteil an TxF führt zu einer Stabilisierung der Reaktion, da eine Temperaturerhöhung die Initiierung reduziert, bzw. diese schon bei niedrigeren Temperaturen einsetzt. Die trotz allem vorhandene hohe Instabilität führt dazu, dass die Temperatur während der Polymerisation ständig durch kleine Druckregelungen manuell konstant gehalten werden muss. Die Schwankungsbreite der Innentemperatur in Tabelle 3.2.1 gibt einen Anhaltspunkt, wie gut dies dem Experimentator gelungen ist. Auch der Druck ist ein Mittelwert, die Standardabweichung beträgt ca. 10 bar.

\subsection{Präparation von zylindrischen Proben}

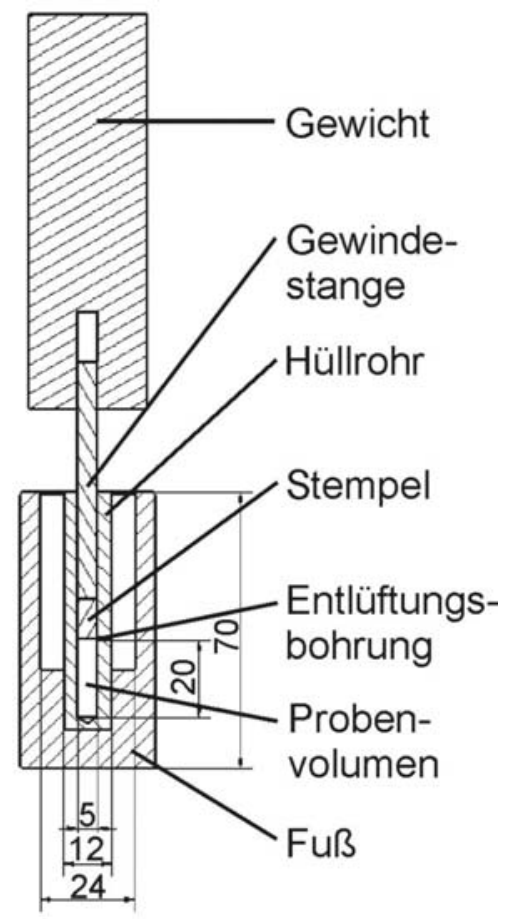

Abb. 3.3.1: EMAA-

Probenpresse, Maße in mm

Für die mechanische Spektroskopie von EMAA-Proben ist es nötig, diese in eine wohl definierte Probenform zu überführen. Eine praktische Geometrie für die Messung der elastischen Eigenschaften von Polymerproben in einem Temperaturbereich von der Flüssig-Stickstofftemperatur bis zum Einsetzen des viskosen Fliesens ist die eines Zylinders [MEN99]. Um diese frei von Blasen herzustellen, erfolgt das Heißpressen unter Vakuum bei Drücken kleiner als $5 \cdot 10^{-5}$ mbar und etwa $390 \mathrm{~K}$ in einer eigens dafür hergestellten Probenpresse. Dazu wird das zerkleinerte Probenmaterial in ein Teflon (PTFE)-Hüllrohr gefüllt und dieses mit einem Stempel aus PTFE verschlossen. Das Hüllrohr wird von einem in das evakuierbare Ofenrohr passenden Fuß aufgenommen und über eine Gewindestange mit einem Stahlgewicht belastet, das eine Kraft von 19,4 N ausübt. Die in den Hohlräumen verbliebene Luft kann beim Evakuieren über eine Entlüftungsbohrung entweichen. Nach Erreichen des Vakuum-Enddrucks wird die Probe $2 \mathrm{~h}$ lang aufgeheizt, wobei sich der Stempel senkt und überschüssiges Polymer durch die Entlüftungsbohrung presst. Ist diese verschlossen, stoppt im Regelfall die Senkbewegung. Diese kann auch durch eine Gewindestange mit variabler Länge eingestellt werden. Die entstandene Probe von etwa $20 \mathrm{~mm}$ Länge und $5 \mathrm{~mm}$ Durchmesser kann nun in der Drehmaschine aus dem Hüllrohr geschält werden. Bei sehr weichen Proben empfiehlt es sich, Hüllrohr und Probe zuvor in flüssigem Stickstoff zu kühlen. Die fertigen Probenzylinder werden zum Schutz vor Wasser in einem Exsikkator aufbewahrt.

\subsection{Rasterelektronenmikroskopie und energiedispersive Röntgen- Mikroanalyse}

Zur Analyse der chemischen Zusammensetzung der metallischen Bänder wird die Methode der energiedispersiven Röntgen-Mikroanalyse (EDX) genutzt. Dabei werden mit dem fokussierten Elektronenstrahl eines Rasterelektronenmikroskops (REM) Elektronen aus den in der Probe enthaltenen Atomen herausgeschlagen und somit angeregt. Das Probenvolumen, in dem Ionisationsereignisse stattfinden, besitzt unterhalb des Auftreffpunkts die Form einer Birne mit einem Durchmesser von $2 \mu \mathrm{m}$. Dies stellt die räumliche Auflösungsgrenze des EDX dar. 
Wenn eine Leerstelle auf einer kernnahen Schale durch ein Elektron aus einem höheren Band wieder gefüllt wird, kommt es zur Emission eines Röntgenphotons mit elementspezifischer Wellenlänge. Die energiedispersive Röntgenanalyse mittels eines $\mathrm{Si}(\mathrm{Li})$-Detektors erlaubt eine quantitative Aussage über die Probenzusammensetzung. Die Kombination mit einem Rasterelektronenmikroskop ermöglicht dabei eine ortsaufgelöste Messung oder die Mittelung über einen festgelegten Bereich, wobei gleichzeitig die Oberfläche der Probe abgebildet wird. Zu diesem Zweck wird ein REM vom Typ Supra 35 der Firma Zeiss mit einem EDX der Firma Thermo Electron eingesetzt. Eine ausführliche Einführung in das Themengebiet REM und EDX geben [GNE92, SL83].

\subsection{Strukturanalyse mittels Weitwinkel-Röntgenbeugung}

Um die Atomstruktur in der Probe zu analysieren, wird die elastische Streuung von Röntgenstrahlung im Bereich großer Streuwinkel (WAXS) genutzt. Das Funktionsprinzip ist dabei recht einfach: Wird Röntgenlicht der Wellenlänge $\lambda$ an zwei Punkten im Abstand $d$ gestreut, so interferieren beide Teilstrahlen konstruktiv, wenn die Bragg-Bedingung erfüllt ist. Ein Intensitätsmaximum der $n$-ten Ordnung ist unter einem Streuwinkel von $2 \Theta$ relativ zum einfallenden Strahl zu beobachten [CUL78].

$$
n \lambda=2 d \sin (\Theta)
$$

Die winkelaufgelöste Verteilung der Streuintensität ermöglicht damit einen Rückschluss auf die Verteilung der Atomabstände in der Probe, wie durch die Paarkorrelationsverteilung $g(r)$ in Kapitel 2.2 beschrieben. Bei einem Kristall weist $g(r)$ scharfe Maxima auf, während diese im Glas aufgrund fehlender Fernordnung sehr breit sind und schnell ganz abnehmen. Dies spiegelt sich auch im Intensitätsverlauf der Röntgenmessung wider. Bei einer amorphen Probe werden breite Intensitätsmaxima der Ordnung $n$ erwartet. Diese treten unter einem Streuwinkel auf, der dem Nächsten-Nachbarabstand entspricht. Bilden sich scharfe Maxima heraus, so ist dies ein Zeichen dafür, dass die Probe zumindest teilweise kristallisiert ist. Nach der Scherrer-Formel [CUL78] kann aus der Breite $B$ der Maxima grob auf den Durchmesser $t$ der Kristalle geschlossen werden.

$$
t=\frac{0,9 \lambda}{B \cos (\Theta)}
$$

Bei diesen Untersuchungen findet ein Diffraktometer des Typs D5000 von Siemens mit einem Zwei-Kreis-Goniometer in Bragg-Brentano-Geometrie Anwendung [HAC03]. Zur Erzeugung der Röntgenstrahlung wird dabei eine Kupferanode verwendet, deren charakteristische Röntgenstrahlung der Linien $\mathrm{K}_{1 \& 2}$ der Wellenlänge $\lambda=0,15418 \mathrm{~nm}$ mittels eines EinkristallMonochromators vor dem Detektor herausgefiltert und somit nutzbar gemacht wird.

\subsection{Differentielle Kalorimetrie}

Die differentielle Kalorimetrie (DSC) wird genutzt, um thermodynamische Zustandsänderungen nachzuweisen. Dazu werden die Probe und eine Referenz mit konstanter Rate aufgeheizt oder abgekühlt. Der Aufbau ist in Abbildung 3.6.1 schematisch dargestellt. Probe und Referenz werden separat beheizt und es wird von beiden die Temperatur gemessen. Durch Anpassung der Ofenleistung mittels eines computergesteuerten Reglers wird versucht für beide die gleiche konstante Heizrate zu fahren. Die differentielle Wärmeaufnahme der Probe im Ver- 
gleich zur Referenz gibt dann eine quantitative Auskunft darüber, ob eine Zustandsänderung stattgefunden hat und wie stark diese die Wärmekapazität der Probe verändert hat. Die Referenz muss dabei so gewählt werden, dass sie im relevanten Temperaturbereich keine $\mathrm{Zu}$ standsänderung und auch keine weiteren Effekte zeigt. Idealerweise sollte sie in etwa die gleiche Wärmekapazität wie die Probe besitzen. Um eine konstante Wärmeankopplung zu erreichen und den Einfluss von wechselnder Luftfeuchtigkeit zu vermeiden, werden die Öfen mit einem konstanten Argon-Strom gespült. Zur Temperaturkalibrierung verwendet man die Schmelzpunkte eines Zink und eines Indium-Standards, letzterer dient auch zur Kalibrierung des Signals des Wärmeflusses.
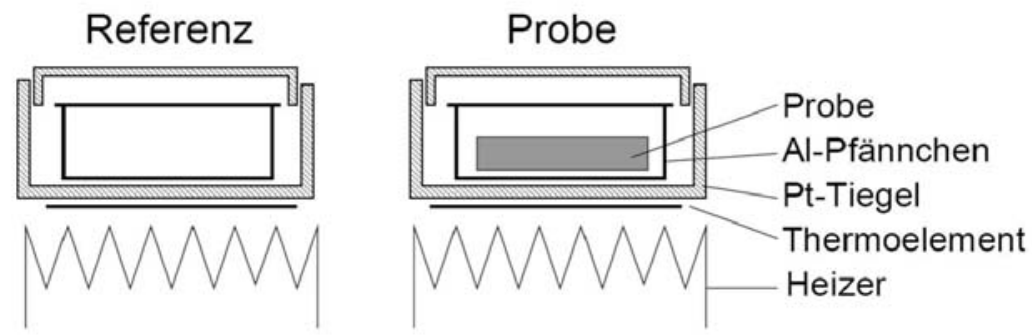

Abb. 3.6.1: Schematischer Aufbau der DSC [WEI98]

Für die Kalorimetrie der metallischen Gläser wird eine DSC 7 der Firma Perkin-Elmer genutzt. Um eine bessere Wärmeankopplung zu erreichen, wurden nicht Probenpfännchen und Deckel benutzt, sondern die Probe zwischen zwei Probenpfännchen verpresst. Diese Methode wurde auch während der Kalibrierung verwendet. Als Referenz für ein amorphes, metallisches Band eignet sich ein zuvor kristallisiertes Stück derselben Probe. Noch einfacher ist es, als Referenz zwei leere Pfännchen zu benutzen, die Probe nach Ende der Messung in der DSC bei hohen Temperaturen zu kristallisieren und das verwendete Zeit-Temperatur-Programm noch einmal auszuführen. Auf diese Weise erhält man eine Basislinie relativ zum kristallinen Zustand mit exakt derselben Probenmasse, die man nur noch von der vorherigen Messung subtrahieren muss.

Auch die Polymerproben können zum Teil in der DSC 7 untersucht werden, allerdings ermöglicht die Glykol-Kühlung keine ausreichend tiefen Anfangstemperaturen, um alle interessanten Phänomene beobachten zu können [MAT94]. Deshalb wird zusätzlich ein Kalorimeter mit Ethanol-Kältemischungs-Kühlung in der Physikalischen Chemie verwendet. Die Starttemperatur liegt damit bei $218 \mathrm{~K}$, was bei einer Heizrate von $5 \mathrm{~K} / \mathrm{min}$ zu stabilen Bedingungen ab etwa $230 \mathrm{~K}$ führt. Ein Nachteil der verwendeten Mettler Toledo DSC 820 ist, dass es sich nicht um eine wie oben beschriebene leistungskompensierte DSC, sondern um eine Wärmefluss-DSC handelt [MAT94], bei der Probe und Referenz im selben Ofen untergebracht sind und nur die Temperaturen separat gemessen werden. Die Leistung wird geregelt, um eine konstante Heizrate zu erreichen. Zusätzlich ist ein Wärmefluss von der Referenz zur Probe oder in die Gegenrichtung möglich. Für eine Quantifizierung der Wärmekapazität würde dies eine Referenz mit im gesamten Temperaturbereich bekannter Wärmekapazität erfordern. Um aussagen zu können, ob eine Zustandsänderung geschieht und ob diese endo- oder exotherm ist, genügt es, ein leeres Pfännchen als Referenz zu benutzen. Eine Vergleichbarkeit von verschiedenen Proben erreicht man durch Subtraktion einer linearen Basislinie im Bereich der 
Flüssigkeit und durch Normierung auf die Probenmasse. Es ist darauf zu achten, dass es dabei noch nicht zu einer thermischen Zersetzung des Materials kommt.

\subsection{Größenausschlusschromatographie}

Die Größenausschlusschromatographie (SEC) dient zur Charakterisierung der Molmassenverteilung. Dazu wird zunächst das Polymer verestert, in Trichlorbenzol gelöst, mit Toluol versetzt und dann bei ca. $393 \mathrm{~K}$ durch eine poröse Säule gespült. Bei der Veresterung wird die Bindungsfähigkeit der Methacrylsäureeinheiten untereinander über Wasserstoffbrückenbindungen unterbunden, was die Löslichkeit erhöht und verhindert, dass das Copolymer sich in der Säule festsetzt (vgl. Abb. 3.2.1). Die Funktionsweise der SEC basiert auf einem einfachen entropischen Argument: Die langkettigen Polymere nehmen mehr Platz ein und können so nur durch die großen Poren der Säule fließen. Kleineren Bestandteilen stehen mehr Poren zur Verfügung und somit auch mehr Möglichkeiten sich zu „verirren“. Am Säulenausfluss werden daher als erstes die hochmolekularen Ketten durch Messung des Brechungsindex nachgewiesen. Das Toluol dient zur Bestimmung eines Referenzzeitpunkts, mit dessen Hilfe die gemessene Verteilung mit einem Polyethylenstandard verglichen und eine quantitative Molmassenverteilung bestimmt werden kann. Eine ausführliche Einführung in die SEC gibt [WU03]. Die SEC-Untersuchungen erfolgten in Zusammenarbeit mit dem Ernst Berl-Institut für Technische und Makromolekulare Chemie der TU Darmstadt.

\subsection{Bestimmung der Zusammensetzung der Polymerproben}

Da es in dieser Arbeit gilt, die makroskopischen Eigenschaften von Copolymeren durch Variation der mikroskopischen, chemischen Begebenheiten $\mathrm{zu}$ untersuchen, ist es wichtig, die chemische Zusammensetzung genau zu kennen. Aus diesem Grund wurden zwei spektroskopische Techniken zur Analyse der chemischen Zusammensetzung eingesetzt, mit denen es prinzipiell auch möglich ist, Aussagen über die Verteilung der Monomere entlang der Kette zu erhalten.

\section{Fourier-Transformierte Infrarotspektroskopie}

Bei der Fourier-transformierten Infrarotspektroskopie (FTIR) handelt es sich um eine Messung der frequenzaufgelösten Absorption von Licht im Infrarotbereich. Die zur Absorption führenden Anregungen in der Probe sind Schwingungen von polaren Atomgruppen, die durch chemische Bindungen verknüpft sind. Die Lage und Form der Absorptionsmaxima als Funktion der Wellenzahl (inverse Wellenlänge, proportional zur Frequenz) ermöglicht eine quantitative Aussage über die chemische Zusammensetzung. Aus der Verschiebung der Maxima erhält man Auskunft über die chemische Nahordnung [IRS95].

Der Aufbau eines FTIR-Spektrometers ist in Abbildung 3.8.1 [HAC03] beschrieben. Die Messung der Absorption erfolgt im gezeigten Aufbau an einem, auf einen reflektierenden Träger aufgebrachten, dünnen Film. Alternativ kann auch eine dünne Folie direkt durchstrahlt werden. Die Schwierigkeit, ein Spektrum nicht direkt frequenzaufgelöst detektieren zu können, wird im FTIR-Spektrometer durch ein gleichmäßig verfahrbares Michelsoninterferometer gelöst. Dabei wird ein kontinuierliches Spektrum einer Quelle Q zeitlich zerlegt und nur jeweils Licht der Frequenzen auf die Probe gegeben, das einen konstruktiven Gangunterschied zwischen den beiden Spiegeln S1 und S2 aufweist. Nun genügt die zeitaufgelöste De- 
tektion D des transmittierten Infrarotlichts. Ein roter Laser bekannter Wellenlänge markiert dabei, in welcher Zeit der verfahrbare Spiegel S2 die Strecke einer Wellenlänge zurückgelegt hat. Die Transformation des zeitlichen Intensitätsverlaufs in den Frequenzraum, bei dem S2 wiederholt um das Vielfache einer Wellenlänge des Kontrolllasers verfahren wird, erfolgt im Computer.

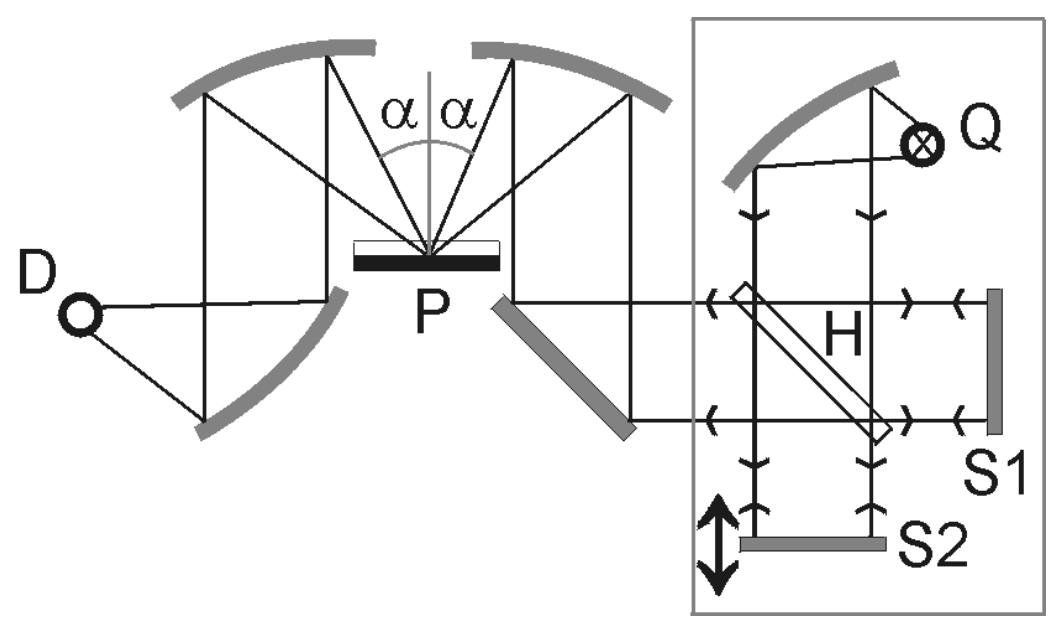

Abb. 3.8.1: Schematischer Aufbau eines FTIR-Spektrometers für Filme auf einem Träger mit vorgeschaltetem Michelsoninterferometer

\section{FTIR in abgeschwächter Totalreflexion}

Bei der FTIR-Analyse wird in der Regel ein homogenes Probenvolumen durchstrahlt, das zu einer ausreichenden Absorption führt. Ist die Absorption zu stark und lässt sich die Probe nicht weiter dünnen oder kommt es zu einer Trübung durch Einschlüsse oder Blässchen, die ein Durchstrahlen verhindern, so kann die Methode der abgeschwächten Totalreflexion (ATR) genutzt werden [STU04]. Dabei wird ein Prisma durchstrahlt, an dessen Oberflächen es mehrfach zu innerer Totalreflexion kommt. Auf eine (oder beide) der Oberflächen ist die zu untersuchende Probe aufgebracht. Im Punkt der Totalreflexion kommt es zu einem Eindringen des Lichts in die Probe, wobei charakteristische Absorption auftritt. Die Eindringtiefe ist dabei im $\mathrm{nm}$-Bereich. Man beobachtet also in den Spektren eventuell Abweichungen durch Oberflächeneffekte zwischen Probe und Prisma. Zur Anwendung kommt dazu ein FTIR-Spektrometer vom Typ Bruker IFS 88 mit einem ATR-Messeinsatz Harrick MVP2 Series.

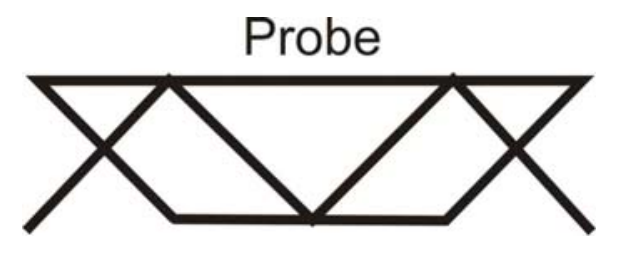

Abb. 3.8.2: Schematischer Aufbau eines ATR-Strahlengangs 


\section{Kernspinresonanz-Spektroskopie}

Die Kernspinresonanz-Spektroskopie (NMR) wird in Zusammenarbeit mit der Arbeitsgruppe um Dr. M. Baldus durchgeführt und dient ebenfalls der Aufklärung der chemischen Zusammensetzung und der Monomerverteilung. Bei der NMR werden in einem starken äußeren Magnetfeld die polarisierbaren Spins $\left({ }^{1} \mathrm{H},{ }^{13} \mathrm{C},{ }^{15} \mathrm{~N}\right.$, etc.) ausgerichtet und dann durch einen elementspezifischen, senkrechten Radiopuls zur Präzession mit ihrer Lamor-Frequenz angeregt. Beim Abklingen dieser angeregten Zustände wird wiederum elektromagnetische Strahlung im MHz-Bereich abgestrahlt und diese detektiert. Eine Fourier-Transformation liefert auch hier das Frequenzspektrum. Eine Eigenart der NMR-Spektroskopie ist, die Abweichungen der Positionen der Maxima relativ zu einem Standard (Tetramethylsilan), geteilt durch die Frequenz des Standards in Millionstel (ppm) anzugeben. Diese Frequenzverschiebung, auch chemische Verschiebung genannt, gibt Aufschluss über die Bindungspartner und die chemische Umgebung der jeweiligen Elemente. Aus den relativen Intensitäten lässt sich die Zusammensetzung gewinnen. Eine genauere Untersuchung des chemischen Aufbaus über mehrere Bindungen hinweg liefern Experimente mit Pulssequenzen, bei denen zunächst ein Kern angeregt, dieser dann zum stimulierten Transfer der Anregung auf einen weiteren Kern veranlasst und abschließend der so angeregte zweite Kern detektiert wird. Dies entspricht einer Kreuzkorrelation der beiden Kernresonanzenspektren, was dem Beobachter verrät, ob die beiden Kerne mit einander koppeln können und wie sie das tun (chemische Bindung oder räumliche Nähe). Diese so genannte zweidimensionale NMR erfordert einige experimentelle Kunstgriffe (Magic Angle Spinning), auf die hier nicht im Detail eingegangen werden soll [MAC98].

\subsection{Dielektrische Spektroskopie}

Die dielektrische Spektroskopie (DES) bietet eine zur mechanischen Spektroskopie alternative Methode der Messung der mechanischen Eigenschaften und der Dynamik in einem nicht leitenden System. Ein schematisiertes Beispiel für die Leistungsfähigkeit dieser Technik zeigt Abbildung 2.5.1. Die Durchführung dieser Methode erfolgt in Zusammenarbeit mit Priv.-Doz. Dr. P. Lunkenheimer und Prof. Dr. A. Loidl am Lehrstuhl für Experimentalphysik V in Augsburg.

Die prinzipielle Funktionsweise ist dabei die folgende [KS03]: Eine Probe wird in ein elektrisches Wechselfeld gebracht und dann die Polarisation des Materials als Funktion der Frequenz gemessen. Elektrisches Feld $\widetilde{E}$ und Polarisation $\widetilde{P}$ sind außer Phase, wenn Relaxationen im Material stattfinden. Die komplexwertige Dielektrizitätszahl $\widetilde{\varepsilon}^{*}$ ist also das Analogon zur Nachgiebigkeit $D^{*}(15)$ :

$$
\begin{aligned}
& \widetilde{P}=\left(\widetilde{\varepsilon}^{*}-1\right) \widetilde{\varepsilon}_{0} \widetilde{E} \\
& \widetilde{\varepsilon}^{*}=\widetilde{\varepsilon}^{\prime}-i \cdot \widetilde{\varepsilon}^{\prime \prime} \\
& \frac{\widetilde{\varepsilon}^{\prime \prime}}{\widetilde{\varepsilon}^{\prime}}=\tan \delta
\end{aligned}
$$

Zur besseren Abgrenzung zum Elastizitätsmodul E und der Dehnung $\varepsilon$ wurden hier alle elektrodynamischen Größen, wie die elektrische Feldkonstante $\widetilde{\varepsilon}_{0}$, mit dem Zeichen $\sim$ gekennzeichnet. 


\subsection{Dynamisch-mechanische Analyse}

Die dynamisch-mechanische Analyse (DMA) dient zur Bestimmung der viskoelastischen Eigenschaften einer makroskopischen Probe. Die verwendete DMA 7 der Firma Perkin-Elmer bietet dazu eine Vielzahl von Geometrien für Proben im festen Zustand. Das Funktionsprinzip ist jedoch stets dasselbe: Eine Probe wird einer statischen, oszillierenden oder sich zeitlich ändernden mechanischen Spannung ausgesetzt und der Verlauf ihrer Deformation beobachtet. Im Folgenden soll die Handhabung der DMA beschrieben werden, mit besonderem Augenmerk auf Methoden, bei denen die Anweisungen im Handbuch [PE94] verändert oder erweitert worden sind, sowie die benutzten Messmodi uniaxiale Dehnung und Kompression. Den Umgang mit der Windows ${ }^{\circledR}$-basierten Software beschreibt die in das Messprogramm integrierte Hilfsfunktion.

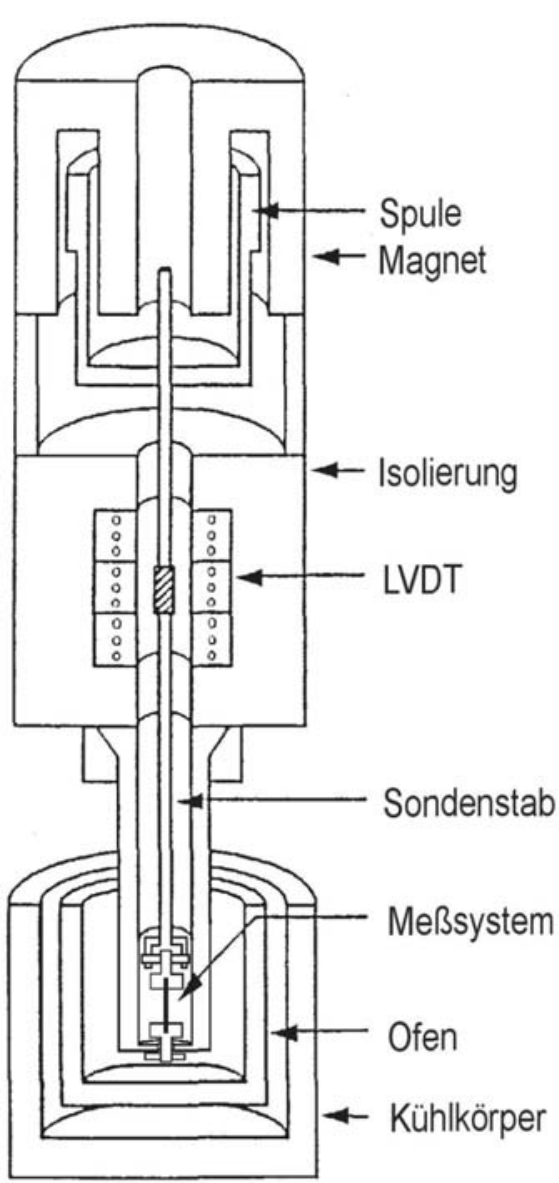

Abb. 3.10.1: Schematischer

Aufbau der DMA

\section{Aufbau}

Der Aufbau der DMA 7 ist in Abbildung 3.10.1 schematisch dargestellt. Die Probe ist in einem variabel wählbaren Meßsystem fixiert und über den Sondenstab mit dem Linearmotor (Spule und Magnet) verbunden. Dieser kann dynamisch mit 0,01 - $51 \mathrm{~Hz}$ oder mit konstanter Kraft von bis zu $1 \mathrm{~N}$ an der Probe ziehen oder diese komprimieren. Beste Ergebnisse lassen sich erzielen im Frequenzbereich zwischen $1-20 \mathrm{~Hz}$ bei Kräften oberhalb $100 \mathrm{mN}$. Die resultierende Amplitude und Dehnung wird mittels eines linear variablen Differential-Transformators (LVDT) detektiert. Dieser arbeitet bei einer konstanten Temperatur von $318 \mathrm{~K}$, um ein Driften des Positionssignals zu minimieren und muss daher zunächst 40 min warmlaufen und gut isoliert werden. Die Probe selbst kann definiert gekühlt oder geheizt werden, wobei ein Argon-Strom im Sondenrohr den LVDT vom Ofen entkoppelt. Die maximal erreichbare Temperatur liegt bei $773 \mathrm{~K}$ im Ofen mit $28 \mathrm{~mm}$ Durchmesser und $1273 \mathrm{~K}$ im Ofen mit $15 \mathrm{~mm}$ Durchmesser. Die Kühlung erfolgt mittels eines GlykolDurchflusskühlers, wobei mit einem Kühler problemlos eine Basistemperatur von $273 \mathrm{~K}$ erreichbar ist. Noch tieferen Temperaturen können mit einer Kühlung durch flüssigen Stickstoff erzielt werden, wobei sich die Glykolküh-

lung durch eine bessere Temperatursteuerung auszeichnet. Bei der Stickstoffkühlung erfolgt die Durchflussreglung automatisch durch ein Steuergerät mit Temperaturfühler vom Typ CCA 7. Zur Vermeidung von Kondenswasser ist es unabdingbar die DMA mit Stickstoffkühlung in einer mit Stickstoff oder Argon gefüllten Handschuhbox zu betreiben. Dies dient zusätzlich zum Argon-Strom im Sondenrohr zur Vermeidung von Oxidationseffekten. Die Kontrolle des Wasser- und Sauerstoffgehalts sowie die Abscheidung dieser Substanzen erfolgt durch eine Anlage vom Typ MB 20G von MBraun. Da in einer wasserarmen Schutzgasatmosphäre (besser als 5 ppm) Siliconschläuche auch ohne mechanische Beanspruchung brechen, 
wird die Stickstoff-Zuleitung durch nicht kunststoffgedichtete Heißwasserschläuche aus Metallgewebe realisiert, die deutlich günstiger sind als die Faltenschlauch-Variante, bei akzeptabler Gasdichtigkeit.

Die Ansteuerung der DMA und das Auslesen der Messgrößen erfolgt durch ein Steuergerät vom Typ TAC 7/DX, das seinerseits mit einem Computer verbunden ist. Die verwendete Version 4 der Pyris Software von Perkin Elmer benötigt dabei zwingend das Betriebssystem Windows 2000 mit Servicepaket 2. Auf diese Weise werden alle programmierbaren Parameter (Kraft, Frequenz, Heizrate) und die detektierten Größen (Änderung und Amplitude des Positionssignals des LVDT, Temperatur) aufgezeichnet. Anregung und Positionsänderung sind bei dynamischen Messungen um den Winkel $\delta$ phasenverschoben. Der Phasenwinkel ist damit direkt messbar, alle anderen Größen, die zur Charakterisierung der viskoelastischen Eigenschaften der Probe berechnet werden, benötigen eine genaue Kenntnis der Probengeometrie und deren Abmessungen.

\section{Kalibrierung}

Die Kalibrierung der DMA erfolgt in sechs Schritten, wobei die Anweisungen der OnlineHilfe der Pyris Software genau befolgt werden sollten. Abweichend oder ergänzend dazu sollte wie folgt verfahren werden:

Bei der „DMA Calibration“ werden neben grundsätzlichen Funktionstests auch die Reibung und die Trägheit der DMA bestimmt. Dazu wird das 3-Punkt-Biegegestänge benutzt. Beim Verwenden von Quarzgestängen für die eigentlichen Messungen sollten auch während der Kalibrierung Quarzgestänge benutzt werden. Des Weiteren ist die Kalibrierung temperaturabhängig und sollte bei der Basistemperatur durchgeführt werden, auch bei der Verwendung der Stickstoffkühlung. Zur Maximierung der Empfindlichkeit des Systems muss zunächst die Reibung minimiert werden. Dazu muss die DMA gerade stehen und der Sondenstab oder Core Rod gerade sein. Das Richten des Core Rods erfolgt außerhalb der DMA in einer optischen Bank durch manuelles, segmentweises Biegen. Anschließend kann die DMA mittels der verstellbaren Standfüße ausgerichtet werden, wobei die Reibung softwaregesteuert überwacht wird. Dazu wird mit montiertem 3-Punkt-Biegegestänge ohne Probe und ohne anliegende Kraft in der Pyris Software die Tastenkombination „Ctrl+Shift+Alt + D“ betätigt. Die Eingabe

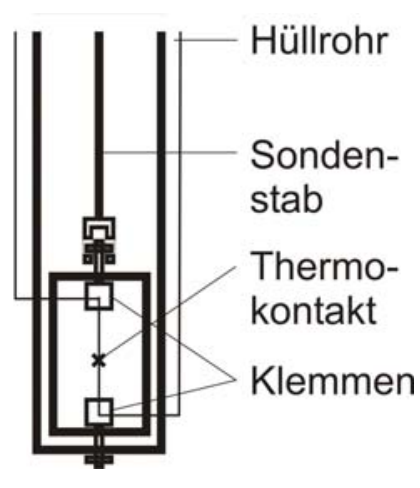

Abb. 3.10.2: Skizze der Kalibrierung mit einem Thermoelement des Befehls „Q,12“ im sich öffnenden Steuerfenster initiiert eine Reibungsmessung, die als Ausgabewert „Q,12,xx“ liefert. xx steht dabei für die Reibung in $\mu \mathrm{N}$. Der Wert muss unter $100 \mu \mathrm{N}$ liegen, um die DMA betreiben zu können. Werte unterhalb $50 \mu \mathrm{N}$ bei Zimmertemperatur sind erstrebenswert.

Die Höhen- und Kraftkalibrierung erfolgen wie im Handbuch beschrieben bei Zimmertemperatur. Da die Eigendeformation temperaturabhängig ist, sollte die Eigendeformationskalibrierung ohne Probe bei der Basistemperatur und mit Stahlzylinder bei Zimmertemperatur erfolgen. Zur Temperaturkalibrierung bieten sich für metallische Proben Zinn und Zink als Kalibriersubstanzen an. Diese reißen beim Erreichen des Schmelzpunktes im Zugversuch ab. Wichtig dabei ist, mit so kleinen Kräften wie möglich zu 
ziehen (ca. $50 \mathrm{mN}$ ), da die Zugfestigkeit bereits vor Erreichen des Schmelzpunktes sinkt, wodurch das Abreißen systematisch bei zu kleinen Temperaturen vonstatten geht. Dieses Verfahren führt zu einer verfälschten Temperaturanzeige im Bereich der Zimmertemperatur. Deshalb wird die Kalibrierung der Temperatur, insbesondere beim Arbeiten mit Stickstoff, durch eine Kalibrierung mit einem Thermoelement am Probenort korrigiert. Den Aufbau zeigt Abbildung 3.10.2. Es ist zu beachten, dass für jede benutzte Heizrate eine Kalibrierung der Temperatur durchgeführt werden muss. Die Ofenkalibrierung zur Verringerung des Unterschieds zwischen Soll- und Ist-Temperatur wird gemäß Handbuch durchgeführt.

\section{Die Messmodi uniaxiale Dehnung und Kompression}

Je nach zu messendem Material bieten sich unterschiedliche Messmodi der DMA an. Eine Einführung, welcher Modus zu welcher Probe passt, gibt [MEN99]. Ist ein metallisches Band bis über den Glasübergang hinaus zu untersuchen, bietet sich die uniaxiale Dehnung an (Abb. 3.10.3 a)), da eine Biegemethode (Abb. 3.10.3 c), d)) nur funktioniert, solange die Probe sich nicht irreversibel verformt. Bei der uniaxialen Dehnung ist ein Fließen der Probe tolerierbar, solange es zu keiner signifikanten Einschnürung der Probe kommt [IRÖ04].

a)

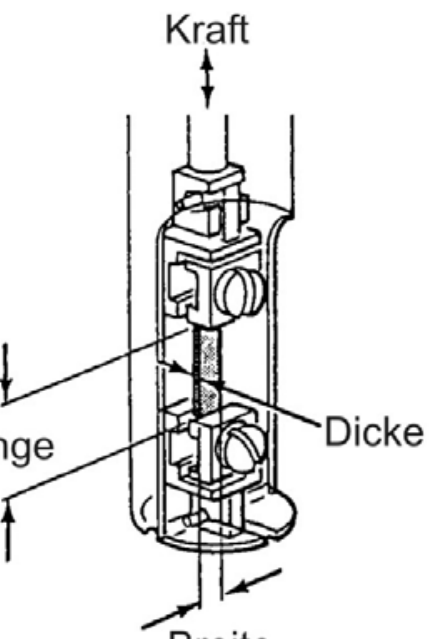

Breite

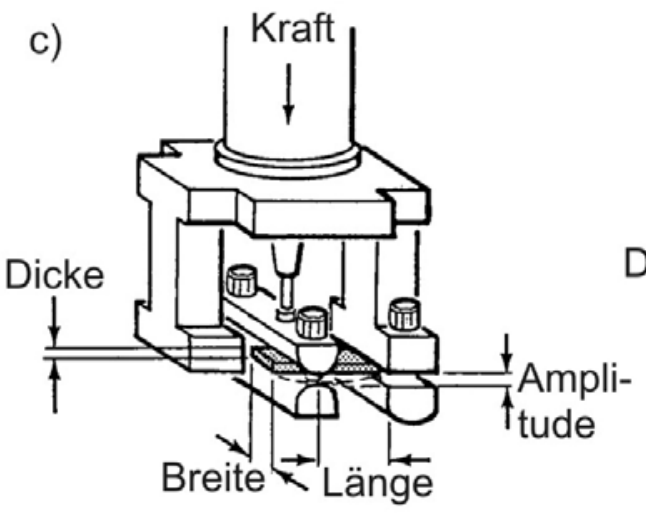

b)

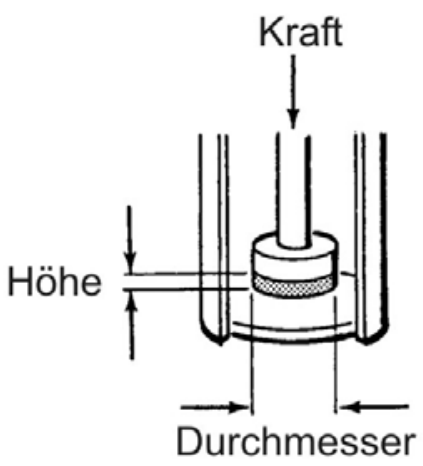

d)

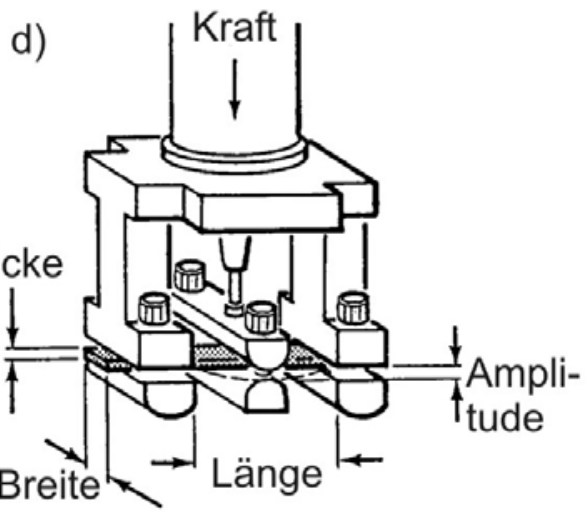

Abb. 3.10.3: Schematische Darstellung der Messmodi: a) Uniaxiale Dehnung, b) Kompression, c) Biegebalken mit einseitiger und d) beidseitiger Fixierung [PE94]

Für ein Polymer bietet sich die Kompression zwischen zwei parallelen Platten an, solange es nicht zu hart ist. Die uniaxiale Dehnung wäre für dünne, homogene Filme anwendbar. Weit 
unterhalb der Glastemperatur wäre auch eine Biegemethode erfolgsversprechend, dies stellt aber hohe Ansprüche an die Qualität eines Films oder Biegebalkens, und der Einbau sollte bei Temperaturen unter der Glastemperatur stattfinden. Aus diesem Grund und wegen der Einsetzbarkeit oberhalb der Glastemperatur wird für EMAA die Kompression zwischen zwei parallelen Platten verwendet (Abb. 3.10.3 b)). 


\section{Untersuchungen an metallischen Gläsern}

\subsection{Analyse der Probenzusammensetzung}

Zur Analyse der Probenzusammensetzung wird die in Kapitel 3.4 beschriebene energiedispersive Röntgen-Mikroanalyse (EDX) verwendet. Untersucht wird die Schnittfläche von Bändern aus metallischem Glas der Zielzusammensetzungen $\operatorname{Pd}_{77,5} \mathrm{Cu}_{6} \mathrm{Si}_{16,5}$ und $\mathrm{Zr}_{65} \mathrm{Al}_{7,5} \mathrm{Cu}_{27,5}$ mit verschiedenen Vorgeschichten, jeweils in der Mitte und in ca. $10 \mu \mathrm{m}$ Entfernung in der Nähe des Randes (Abb. 4.1.1). Dazu wird $200 \mathrm{~s}$ lang ein ca. 6 x $5 \mu \mathrm{m}$ großes Feld auf der Probenoberfläche mit dem Elektronenstrahl abgerastert, wobei die Beschleunigungsspannung $20 \mathrm{kV}$ beträgt. Die emittierte, charakteristische Röntgenstrahlung wird quantitativ und energieaufgelöst detektiert und die Sekundärelektronen zur Bilderzeugung genutzt.

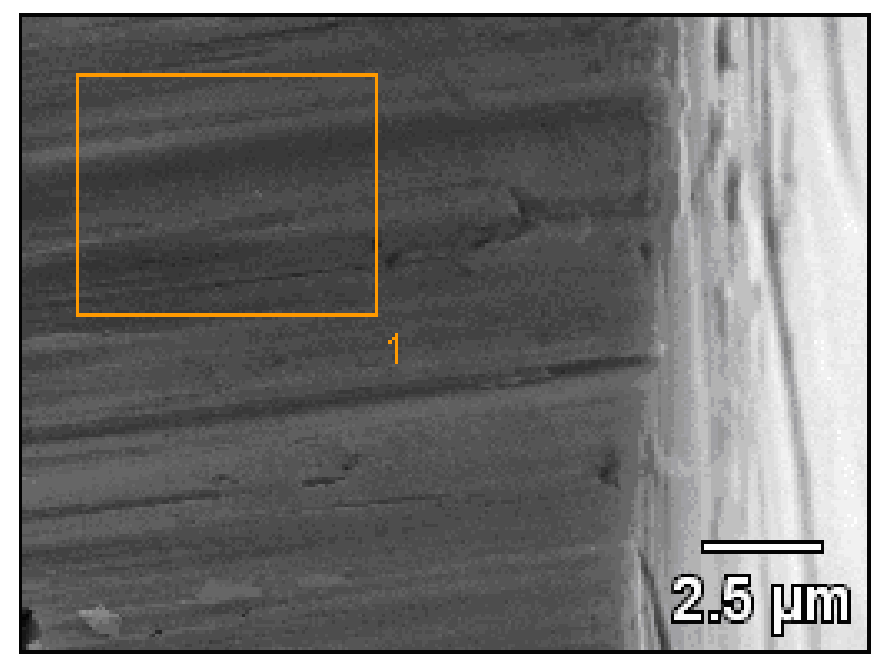

Abb. 4.1.1: Beispiel für die Ortswahl der EDX-Analyse anhand der ZrAlCu-Ausgangsprobe

Tab. 4.1.1: Auflistung der Ergebnisse der EDX-Analyse an drei unterschiedlich vorbehandelten PdCuSi-Proben

\begin{tabular}{|l|l|l|l|l|}
\hline Probe, Vorgeschichte und Ort & $\mathrm{Pd}[\mathrm{at} \%]$ & $\mathrm{Cu}[\mathrm{at} \%]$ & $\mathrm{Si}[\mathrm{at} \%]$ & $\mathrm{O}[\mathrm{at} \%]$ \\
\hline Zielzusammensetzung & 77,5 & 6,0 & 16,5 & - \\
\hline Ausgangsprobe, Mitte & 68,5 & 5,4 & 13,9 & 12,2 \\
\hline Ausgangsprobe, Rand & 71,5 & 5,9 & 15,2 & 7,4 \\
\hline Angelassen bei 627 K, Mitte & 74,8 & 5,8 & 15,8 & 3,6 \\
\hline Angelassen bei 627 K, Rand & 74,0 & 5,7 & 15,4 & 4,9 \\
\hline Kristallisiert, Mitte & 76,6 & 5,9 & 15,3 & 2,2 \\
\hline Kristallisiert, Rand & 76,1 & 6,2 & 15,3 & 2,4 \\
\hline Mittelwert mit O & 73,6 & 5,8 & 15,1 & 5,5 \\
\hline Standardabweichung & 3,1 & 0,3 & 0,7 & 3,9 \\
\hline Mittlere Zusammensetzung ohne O & 77,8 & 6,2 & 16,0 & - \\
\hline
\end{tabular}


Die ermittelte Zusammensetzung der PdCuSi-Probe zeigt Tabelle 4.1.1. Proben, die nur vorlegiert und rasch abgeschreckt wurden, nicht aber thermisch oder mechanisch weiterbehandelt, werden Ausgangsproben genannt. Als angelassen werden Proben bezeichnet, die zuvor mit $1 \mathrm{~Hz}$ und $3 \mathrm{~K} / \mathrm{min}$ bis zu der angegebenen Temperatur spektroskopisch untersucht worden sind, worauf genauer in Kapitel 4.6 eingegangen wird. Es wurde in allen Proben als einzige Verunreinigung Sauerstoff $(\mathrm{O})$ mit einer mittleren Konzentration von 5,5 at $\%$ gefunden, wobei die Standardabweichung 3,9 at\% beträgt. Die mittlere Zusammensetzung mit Sauerstoff ist in Tabelle 4.1.1 angegeben. Da eine Kontamination mit Sauerstoff nicht völlig zu vermeiden ist, wird die Zusammensetzung ohne Sauerstoff zu Pd: 77,8 at\%, Cu: 6,2 at\% und Si: 16,0 at\% bestimmt. Dies stimmt bis auf einen Fehler im Prozentbereich mit der Zielzusammensetzung $\mathrm{Pd}_{77,5} \mathrm{Cu}_{6} \mathrm{Si}_{16,5}$ überein. Ein systematischer Zusammenhang mit der Vorgeschichte ist nicht zu erkennen.

Tab. 4.1.2: Auflistung der Ergebnisse der EDX-Analyse an drei unterschiedlich vorbehandelten $\mathrm{ZrAlCu}$-Proben

\begin{tabular}{|l|l|l|l|l|}
\hline Probe, Vorgeschichte und Ort & $\mathrm{Zr}[\mathrm{at} \%]$ & $\mathrm{Al}[\mathrm{at} \%]$ & $\mathrm{Cu}[\mathrm{at} \%]$ & $\mathrm{O}[\mathrm{at} \%]$ \\
\hline Zielzusammensetzung & 65,0 & 7,5 & 27,5 & - \\
\hline Ausgangsprobe, Mitte & 64,5 & 7,7 & 26,6 & 1,2 \\
\hline Ausgangsprobe, Rand & 63,0 & 7,7 & 26,7 & 2,6 \\
\hline Angelassen bei 665 K, Mitte & 62,7 & 6,5 & 26,2 & 4,6 \\
\hline Angelassen bei 665 K, Rand & 61,5 & 6,4 & 25,6 & 6,5 \\
\hline Kristallisiert, Mitte 1 & $(62,5)$ & $(11,8)$ & $(21,6)$ & $(4,1)$ \\
\hline Kristallisiert, Mitte 2 & $(61,9)$ & $(12,5)$ & $(21,3)$ & $(4,3)$ \\
\hline Kristallisiert, Mitte 2'* $*$ & $(63,2)$ & $(10,6)$ & $(22,1)$ & $(4,1)$ \\
\hline Kristallisiert, Rand & $(61,9)$ & $(12,7)$ & $(21,7)$ & $(3,7)$ \\
\hline Mittelwert mit O, alle & 62,6 & 9,5 & 24,0 & 3,9 \\
\hline Standardabweichung, alle & 0,9 & 2,7 & 2,5 & 1,5 \\
\hline $\begin{array}{l}\text { Mittlere Zusammensetzung ohne O, } \\
\text { alle }\end{array}$ & 65,2 & 9,9 & 24,9 & - \\
\hline $\begin{array}{l}\text { Mittelwert mit O, } \\
\text { ohne kristalline Probe }\end{array}$ & 62,9 & 7,1 & 26,3 & 3,7 \\
\hline $\begin{array}{l}\text { Standardabweichung, } \\
\text { ohne kristalline Probe }\end{array}$ & 1,2 & 0,7 & 0,5 & 2,3 \\
\hline $\begin{array}{l}\text { Mittlere Zusammensetzung ohne O, } \\
\text { ohne kristalline Probe }\end{array}$ & 65,4 & 7,4 & 27,2 & - \\
\hline
\end{tabular}

*: Bei "Kristallisiert, Mitte 2\%" handelt es sich um eine Punktmessung, $1 \mu \mathrm{m}$ außerhalb der Messung "Kristallisiert, Mitte 2".

Auch bei den ZrAlCu-Proben (Tab. 4.1.2) ist eine Sauerstoffkontamination im Bereich von 3,9 at $\%$ und einer Standardabweichung von 1,5 at\% festzustellen. Bei der Zusammensetzung 
aus den legierten Materialien ist zu sehen, dass die kristallisierten Proben von den angelassenen und unbehandelten Proben abweichen. Der Al-Anteil ist im Wesentlichen auf Kosten des $\mathrm{Cu}$-Gehalts erhöht, gemessen an drei verschieden Orten, wobei das Messfeld Mitte 1 nur ca. $10 \mu \mathrm{m}$ von der Messung am Rand entfernt ist. Die Messungen Mitte 2 und 2' wurden aber in Entfernung von einigen $100 \mu \mathrm{m}$ gemessen. Bei 2' handelt es sich zudem um eine Punktmessung, ca. $1 \mu \mathrm{m}$ außerhalb des Feldes der Messung 2. Sie zeigt jedoch ein vergleichbares Ergebnis zu den ansonsten analysierten Rasterfeldern. Aufgrund der thermischen Vorbehandlung bis zur Kristallisation sind die Mittelwerte getrennt dargestellt. Unter Vernachlässigung der kristallisierten Probe und ohne Berücksichtigung des Sauerstoffanteils wird die Zusammensetzung zu Zr: 65,4 at\%, Al: 7,4 at\% und $\mathrm{Cu}$ : 27,2 at\% bestimmt, die wiederum bis auf einen Abweichung von wenigen Prozent mit der Zielzusammensetzung $\mathrm{Zr}_{65} \mathrm{Al}_{7,5} \mathrm{Cu}_{27,5}$ übereinstimmt.

\subsection{Ergebnisse der Strukturanalyse}

Die Struktur der metallischen Bänder wird mittels Weitwinkel-Röntgenbeugung (WAXS) in Bezug auf das eventuelle Vorhandensein einer wie in Kapitel 2.2 beschriebenen Korrelation der Atomverteilung untersucht. Die winkelaufgelöste Intensitätsverteilung der Streuexperimente (siehe Kapitel 3.5) an einem PdCuSi- und einem ZrAlCu-Band ohne zusätzliche thermische oder mechanische Vorbehandlung (Ausgangsproben) zeigen die Abbildungen 4.2.1 und 4.2.2:

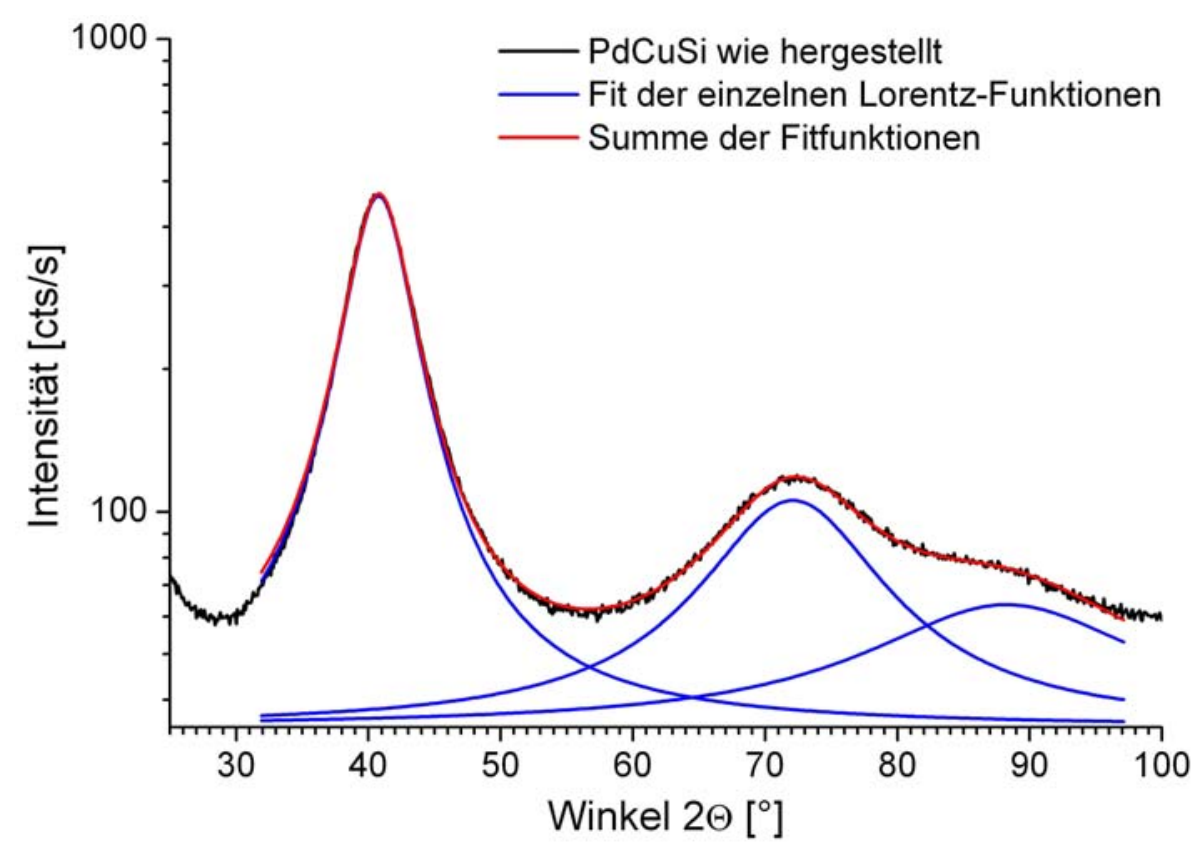

Abb. 4.2.1: WAXS an einer PdCuSi-Ausgangsproben, angepasst mit drei Lorentzfunktionen

Die Messung erfolgte unter den folgenden Rahmenbedingungen: Zunächst werden Streifen von etwa $1 \mathrm{~cm}$ Länge von mehreren Stellen des unbehandelten, wie hergestellten Bandes auf ein doppelseitiges Klebeband aufgebracht und bei kleinen Blendenöffnungen (Blende $1 \& 2$ : $0,1 \mathrm{~mm}$ ) und unter Zuhilfenahme eines fluoreszierenden Phosphorschirms in der Mitte des Strahls platziert. Die eigentliche Messung erfolgt mit großen Blenden (Blende 1 - 3: $1 \mathrm{~mm}$, Blende 4: $2 \mathrm{~mm}$ ) schrittweise im Winkelbereich von $20^{\circ}<2 \Theta<100^{\circ}$, mit einer Schrittweite 
von $\Delta 2 \Theta=0,1^{\circ}$ und einer Bestrahlungszeit von $65 \mathrm{~s} /$ Schritt im Messmodus mit der Bedingung Einfallswinkel gleich Ausfallswinkel. In der Intensitätsverteilung sind keine scharfen Spitzen zu beobachten, sondern ausschließlich breite Maxima bei 40,78(1) ${ }^{\circ}, 72,10(6)^{\circ}$ und 88,26(28) ${ }^{\circ}$, wobei letzteres als Schulter am Rande des zweiten Maximums erscheint. Die Lage der Maxima wurde dabei mit einem Fit aus drei Lorentz-Funktionen bestimmt.

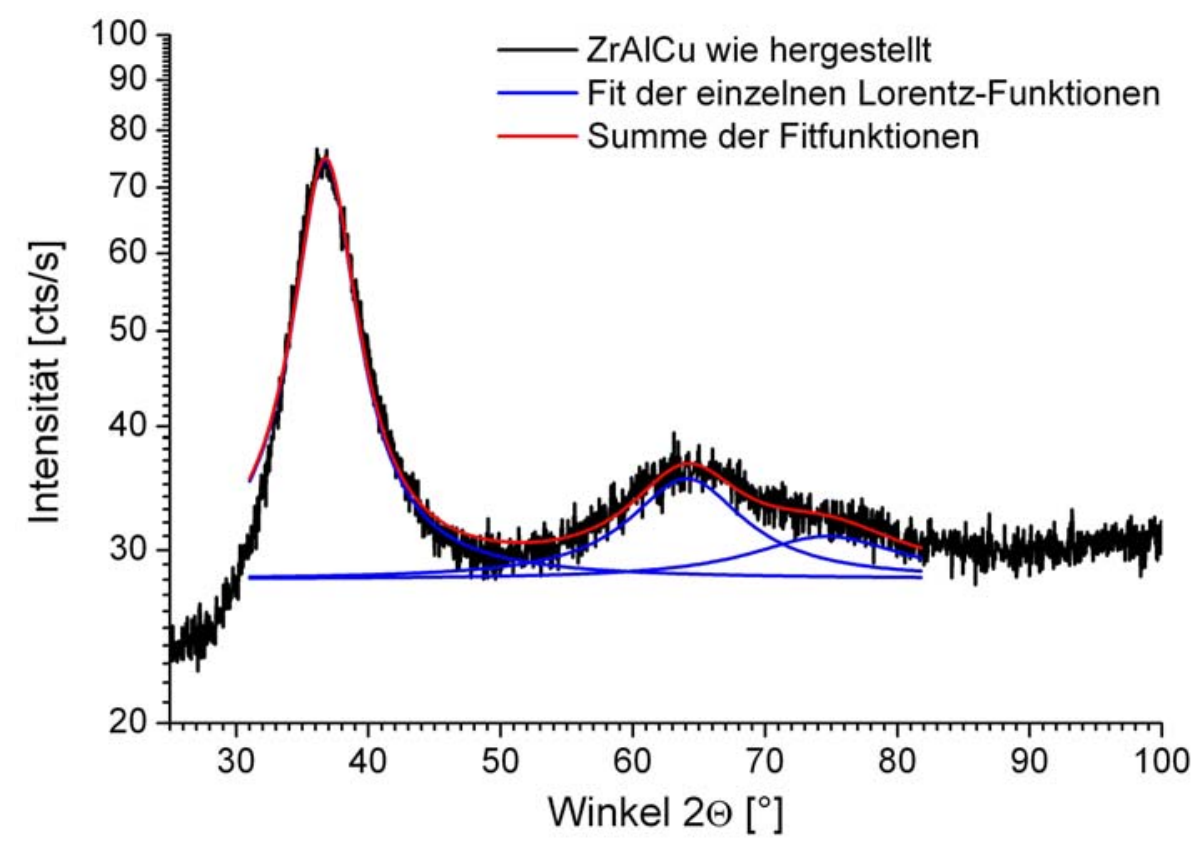

Abb. 4.2.2: WAXS an einer $\mathrm{ZrAlCu}$-Ausgangsprobe, angepasst mit drei Lorentzfunktionen

Die Messung an der $\mathrm{ZrAlCu}$-Ausgangsprobe erfolgte in einer vergleichbaren Weise, allerdings mit einer Schrittweite von $\Delta 2 \Theta=0,05^{\circ}$ und einer Bestrahlungszeit von $40 \mathrm{~s} / \mathrm{Schritt}$. Dies erklärt die schlechtere Statistik, ein Ausschluss von scharfen Spitzen ist dennoch möglich. Es sind wiederum drei breite Maxima bei 36,73(2) ${ }^{\circ}, 64,06(21)^{\circ}$ und $74,66(76)^{\circ} \mathrm{zu}$ beobachten, wobei letzteres wiederum teilweise vom zweiten Maximum überdeckt wird, was eine genaue Anpassung der Daten erschwert. Der Fehler in der Bestimmung des dritten Maximums liegt bei einer manuellen Abschätzung bei eher $2{ }^{\circ}$.

Wie bei der EDX-Analyse in Kapitel 4.1 werden auch hier zusätzlich ausgelagerte Proben untersucht. Es handelt sich dabei um genau dieselben Proben, die zuvor mit $1 \mathrm{~Hz}$ und $3 \mathrm{~K} / \mathrm{min}$ bis zu der angegebenen Temperatur spektroskopisch untersucht worden sind (vergleiche Kapitel 4.6). Dazu werden die folgenden Messparameter benutzt: $2 \Theta=25-60^{\circ}, \Delta 2 \Theta=0,05^{\circ}$ und einer Bestrahlungszeit von $80 \mathrm{~s} /$ Schritt. Die Blenden bleiben unverändert. Die Messung erfolgt an nur einem, wie oben beschrieben vorbehandelten Streifen. Deshalb trifft der Röntgenstrahl weniger Probe und mehr doppelseitiges Klebeband, was eine längere Bestrahlung erforderlich macht, um eine befriedigende Statistik zu erreichen. Es sind wiederum dieselben, breiten Maxima zu sehen, wie in Abbildung 4.2.3 gezeigt, und einen Anstieg zu kleinen Winkeln. Zusätzlich zeigen die ZrAlCu-Proben ein kleines schärferes Maximum bei ca. $2 \Theta=30,3^{\circ}$, das von der Röntgen-Evaluierungs-Software "EVA MFC 4.0.0.2“ als Zirkoniumoxid $\left(\mathrm{ZrO}_{2}\right)$ identifiziert wird. Nach einem Abschmirgeln der während des Anlassens matt gewordenen Oberfläche verliert dieses Maximum deutlich an Intensität. 


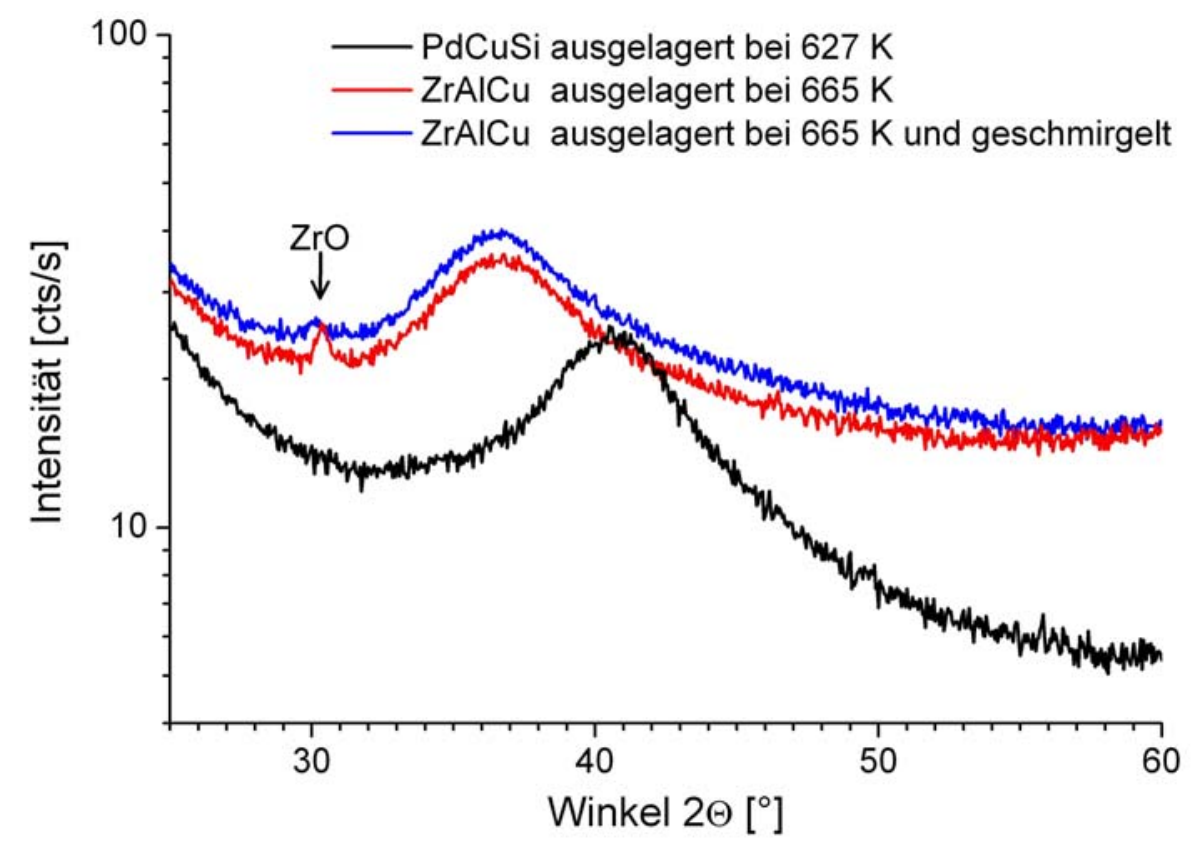

Abb. 4.2.3: WAXS an ausgelagerten PdCuSi- und ZrAlCu-Bändern

\subsection{Kalorimetrische Untersuchungen}

Einen ersten Überblick über die im Material stattfindenden Umwandlungen und deren Wärmetönung gibt die differentielle Kalorimetrie (DSC), wie in Kapitel 3.6 einführend beschrieben. Abbildung 4.3.1 zeigt die bei einer Messung an PdCuSi auftretenden Effekte in einem Temperaturbereich von $576-720 \mathrm{~K}$ bei einer Heizrate von $5 \mathrm{~K} / \mathrm{min}$ :

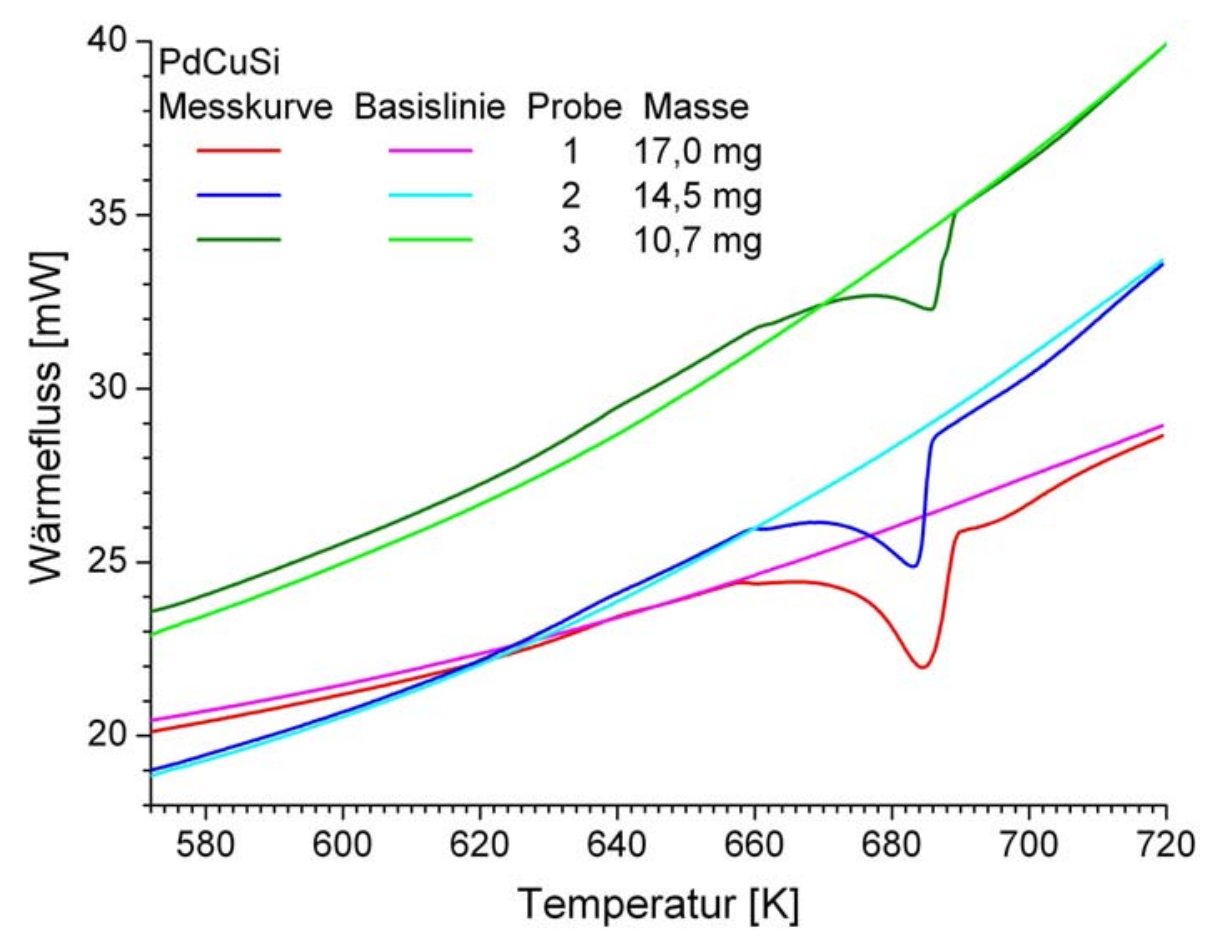

Abb. 4.3.1: DSC-Messung bei $5 \mathrm{~K} / \mathrm{min}$ an PdCuSi-Proben unterschiedlicher Masse und deren Basislinien

Aufgetragen ist als Funktion der Temperatur der Wärmefluss dreier Proben mit verschiedenen Massen zwischen 10,7 mg und 17,0 mg sowie deren Basislinien. Als Referenz wurden zwei 
leere, verpresste Aluminiumtiegel verwendet. Bei den Basislinien handelt es sich um ein zweites Messen der Probe unter gleichen Bedingungen und unter Verwendung derselben Programmprozedur. Es ist in Abbildung 4.3.1 zu erkennen, dass die Messkurve und die zugehörige Basislinie nicht gerade verlaufen, sondern einen mehr oder weniger steilen, konvexen Anstieg vollführen. Dieser Anstieg ist vergleichbar für die Messkurven und deren Basislinien, unterscheidet sich aber von Messkurve zu Messkurve. Des Weiteren werden charakteristische Unterschiede zwischen Messkurve und zugehöriger Basislinie beobachtet, deren Stärke mit der Probenmasse skaliert. Da nur diese für das System charakteristischen Effekte von Interesse sind, wird im Folgenden eine wie oben beschrieben gewonnene Basislinie von der Messkurve abgezogen und die Differenz auf die Masse normiert. Anschließend wird zur besseren Vergleichbarkeit der erste Wert der normierten Wärmeflussdifferenz auf null gesetzt. Abbildung 4.3.2 zeigt eine so berechnete Messkurve der PdCuSi-Ausgangsproben, die auch in Abbildung 4.3.1, ebenfalls als Probe 1 bezeichnet, gezeigt wird. Diese wird mit zwei weiteren Proben verglichen, die zuvor bei einer konstanten Temperatur von $616 \mathrm{~K}$ für 344 min bzw. 502 min ausgelagert worden sind.

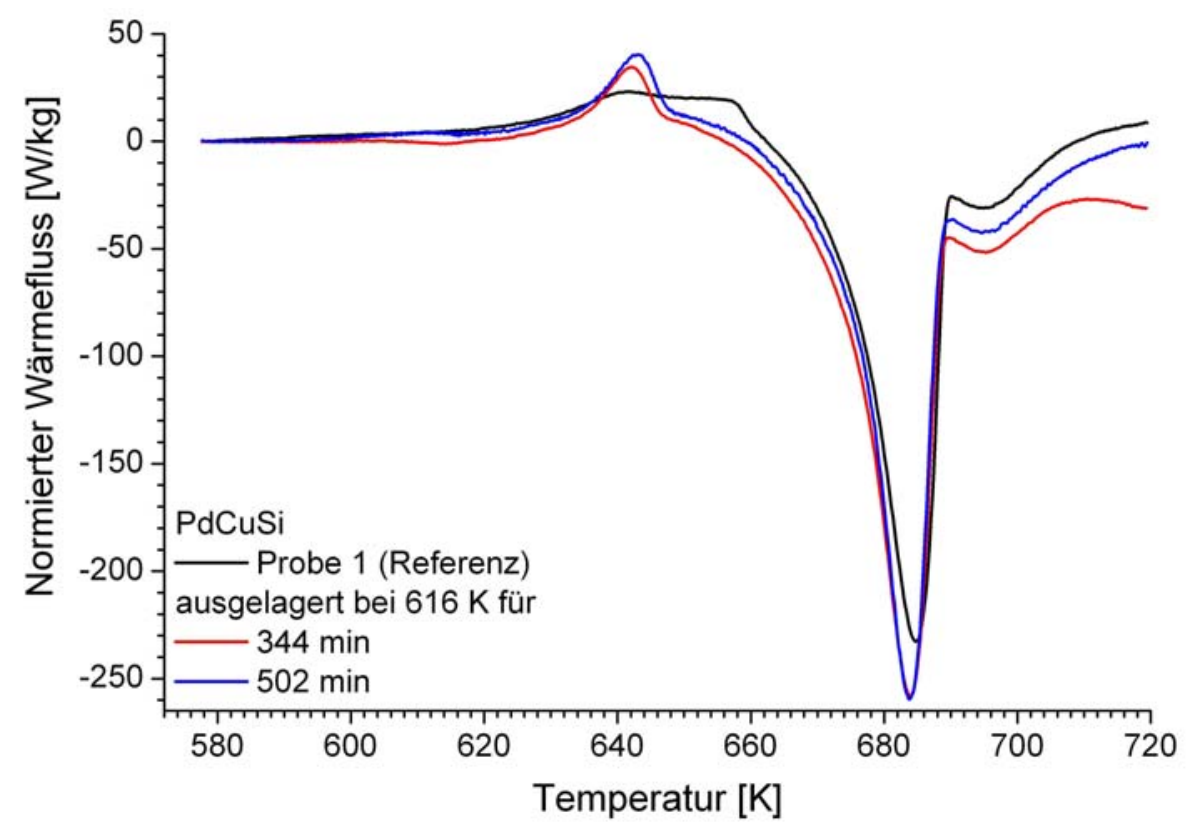

Abb. 4.3.2: DSC-Messung an bei $616 \mathrm{~K}$ ausgelagerten PdCuSi-Proben und einer Referenzmessung

Nach einem nahezu konstanten Verlauf ist oberhalb von $620 \mathrm{~K}$ einen klaren Anstieg hin zu einem, besonders bei den ausgelagerten Proben ausgeprägten, endothermen Maximum zu erkennen, an das sich ein Plateau oder ein Gebiet mit einem schwachen Abfall anschließt. Bei ca. $659 \mathrm{~K}$ setzt ein starker Abfall ein, der in einem stark exothermen Minimum oberhalb $682 \mathrm{~K}$ mündet. Auf einen weiteren Anstieg folgt ein schwächer ausgeprägtes Minimum bei etwa 695 K. Da besonders der Bereich des ersten, endothermen Maximums von Interesse ist, zeigt Abbildung 4.3.3 diesen Ausschnitt in Vergrößerung. Den Beginn des Anstiegs zum Maximum trägt, aus dem Englischen übersetzt, die Bezeichnung Onset-Punkt. Dieser wird aus dem Schnittpunkt der Tangenten an die Kurve gewonnen. Eine weitere Quantisierungsmöglichkeit des Anstiegs ist die Angabe des Wendepunkts, der sich in der Temperaturablei- 
tung des normierten Wärmeflusses bestimmen lässt. Onsets und Wendepunkte sind mit Pfeilen markiert. Tabelle 4.3.1 listet diese Punkte der gezeigten Messungen auf. In der Vergrößerung erkennt man weiterhin, dass die Ausgangsprobe bis zum Onset kontinuierlich ansteigt, dass der Anstieg weniger abrupt beginnt und auch dass die Höhe des Maximums über dem folgenden Bereich des Sattels niedriger ist. Sowohl der Onset als auch der Wendepunkt werden mit steigender Auslagerungszeit zu höheren Werten verschoben. Weiterhin scheint der Bereich vor dem Anstieg bei den ausgelagerten Proben mehr Struktur zu besitzen: Bei ca. $605 \mathrm{~K}$ bzw. $612 \mathrm{~K}$ ist ein schwach ausgeprägtes Maximum zu beobachten.

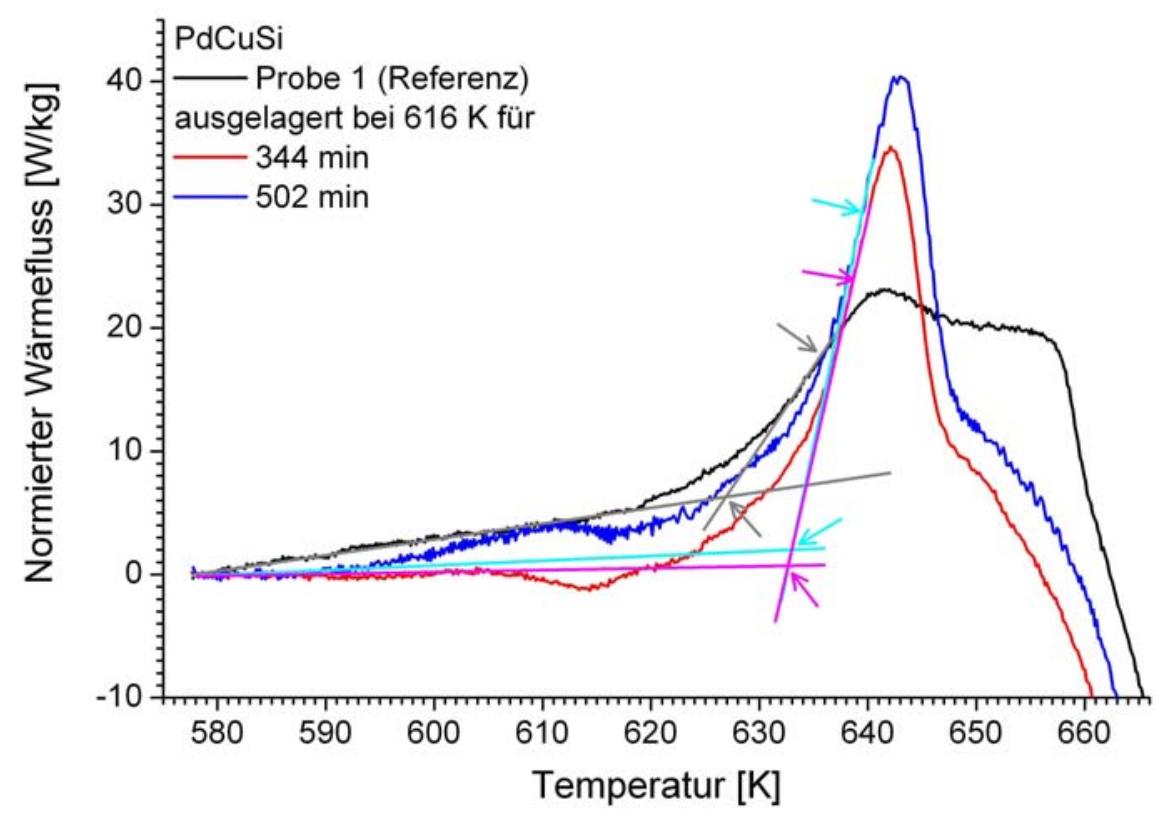

Abb. 4.3.3: Ausschnittsvergrößerung mit Wendepunkten und Tangenten zur Bestimmung des Onsets

Tab. 4.3.1: Tabelle der Onsets und Wendepunkte der DSC-Messungen bei $5 \mathrm{~K} / \mathrm{min}$

\begin{tabular}{|l|l|l|}
\hline Probenbezeichnung & Onset $[\mathrm{K}]$ & Wendepunkt $[\mathrm{K}]$ \\
\hline Wie hergestellt: & & \\
\hline Probe 1 & $627(3)$ & $635(3)$ \\
\hline Probe 2 & $626(3)$ & $633(3)$ \\
\hline Probe 3 & $625(3)$ & $635(3)$ \\
\hline Ausgelagert bei 616 K für & & \\
\hline 344 min & $632(3)$ & $639(1)$ \\
\hline 502 min & $633(3)$ & $640(1)$ \\
\hline
\end{tabular}

Die vorangegangenen Messungen zeigen Messkurven oberhalb $576 \mathrm{~K}$ bei einer Heizrate von $5 \mathrm{~K} / \mathrm{min}$. Bei Zimmertemperatur beginnend, hat es sich zur Zeitersparnis als praktisch erwiesen, zunächst mit einer höheren Rate von $40 \mathrm{~K} / \mathrm{min}$ auf $573 \mathrm{~K}$ zu heizen und dann der DSC bei dieser Temperatur etwas Zeit zum Einpendeln zu gewähren. 
Während dieser Zeit bei nahezu konstanter Temperatur ist der Wärmefluss nur bei den bereits bei höheren Temperaturen ausgelagerten Proben nahezu stabil oder leicht fallend, während die Ausgangsproben ein exponentielles Kriechen auf einen Plateauwert zeigen.

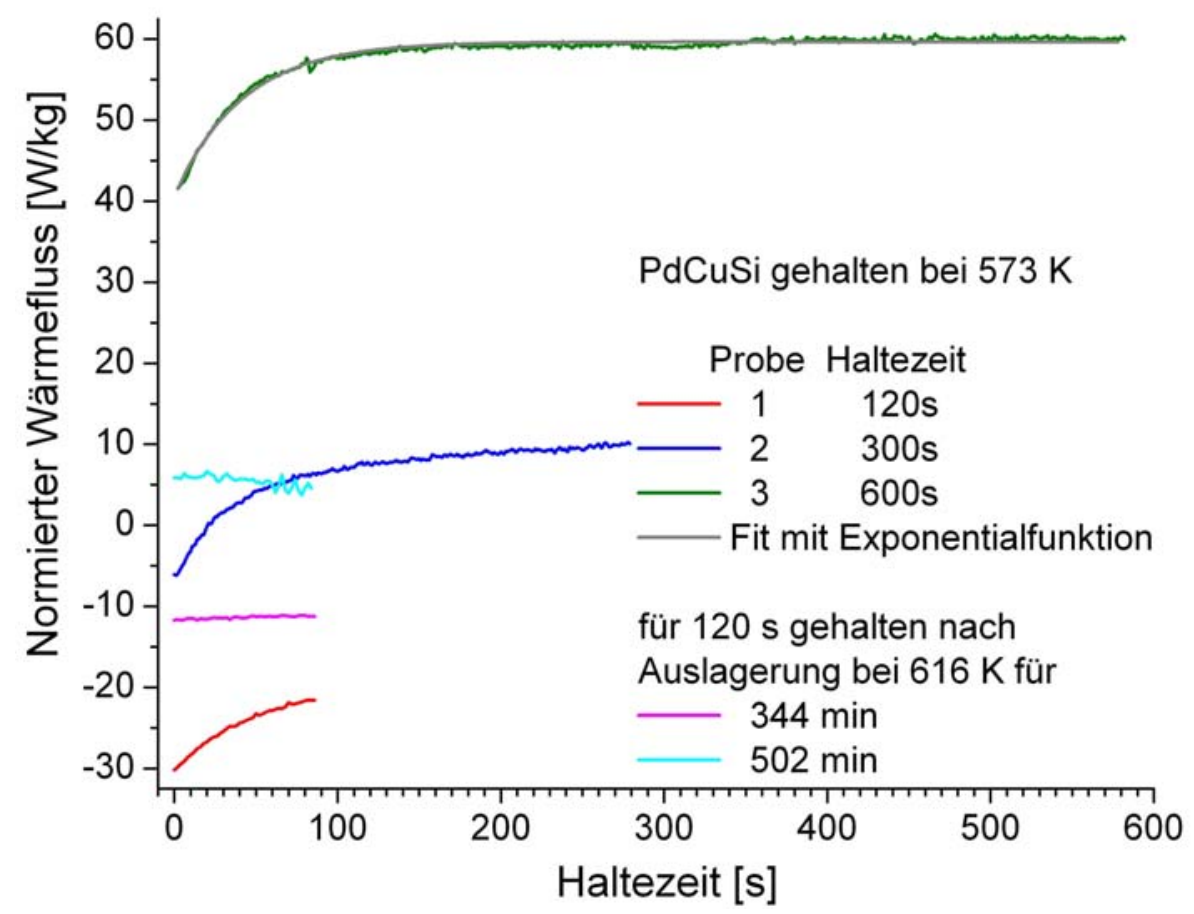

Abb. 4.3.4: Normierter Wärmefluss während des Haltens bei $573 \mathrm{~K}$

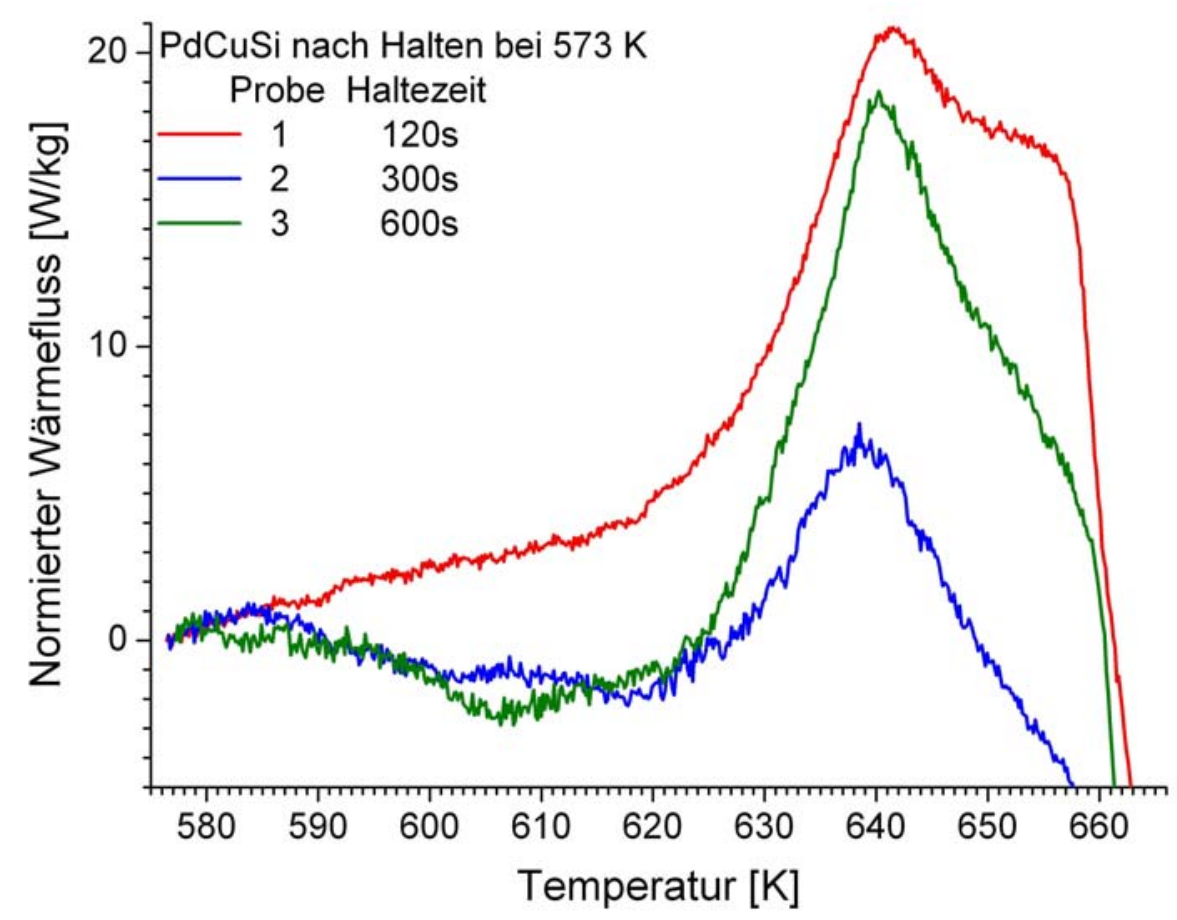

Abb. 4.3.5: Auswirkungen des Haltens bei $573 \mathrm{~K}$ auf die DSC-Messungen

Für die längste Haltezeit beträgt die charakteristische Zeit des in Grau gezeigten Fits 41,6(5) s. Ist die Haltezeit bei $573 \mathrm{~K}$ länger als 120 s, wie im Fall von Probe 1, so wird auch bei dieser niedrigeren Auslagerungstemperatur im Anschluss ein deutlicher ausgeprägtes Ma- 
ximum oberhalb $640 \mathrm{~K}$ und ein breites Maximum unterhalb $610 \mathrm{~K}$ beobachtet, wie in Abbildung 4.3.5 gezeigt.

\subsection{Dynamisch-mechanische Spektroskopie an PdCuSi}

\section{Messung und Cole-Cole-Anpassung der $\alpha$-Relaxation von PdCuSi}

Die mechanischen Eigenschaften der metallischen Gläser werden dynamisch bei einer festen Frequenz und konstanten Heizrate mit Hilfe der in Kapitel 3.10 beschriebenen DMA 7 in uniaxialer Dehnung gemessen. Dabei stehen im Wesentlichen zwei Modi zur Verfügung: Entweder erfolgt die Messung bei einer konstanten statischen und einer konstanten dynamischen Kraft, oder die Soll-Amplitude wird vorgegeben, welche dann computergesteuert durch Anpassung der dynamischen Kraft gehalten wird. Bei der Wahl der statischen Kraft ist darauf zu achten, dass diese zu jeder Zeit des Experiments deutlich größer ist als die dynamische, da sonst die Klemmen zusammenprallen, was die Quarzhaken zerstören kann. Bei großen Kräften ist zu erwarten, dass die Messergebnisse selbst kraftabhängig werden [DHT06]. Dann spielt auch das Verhältnis von statischer zu dynamischer Kraft eine wichtige Rolle. Diese Abhängigkeit wird im zweiten Teil dieses Unterkapitels zusammen mit Effekten durch die Variation anderer Messparameter behandelt.

Abbildung 4.4.1 zeigt die von der DMA aufgezeichneten Rohdaten einer typischen Messung an PdCuSi bei der Frequenz $1 \mathrm{~Hz}$, Heizrate $5 \mathrm{~K} / \mathrm{min}$, konstanter Kraft von $250 \mathrm{mN}$ und dynamischer Kraft von $200 \mathrm{mN}$. Vor Beginn der Messung mit $20 \mathrm{~K} / \mathrm{min}$ wird auf $573 \mathrm{~K}$ geheizt und diese Soll-Temperatur 4 min gehalten, währenddessen sich die Probentemperatur stabilisiert und Anfangseffekte abklingen.

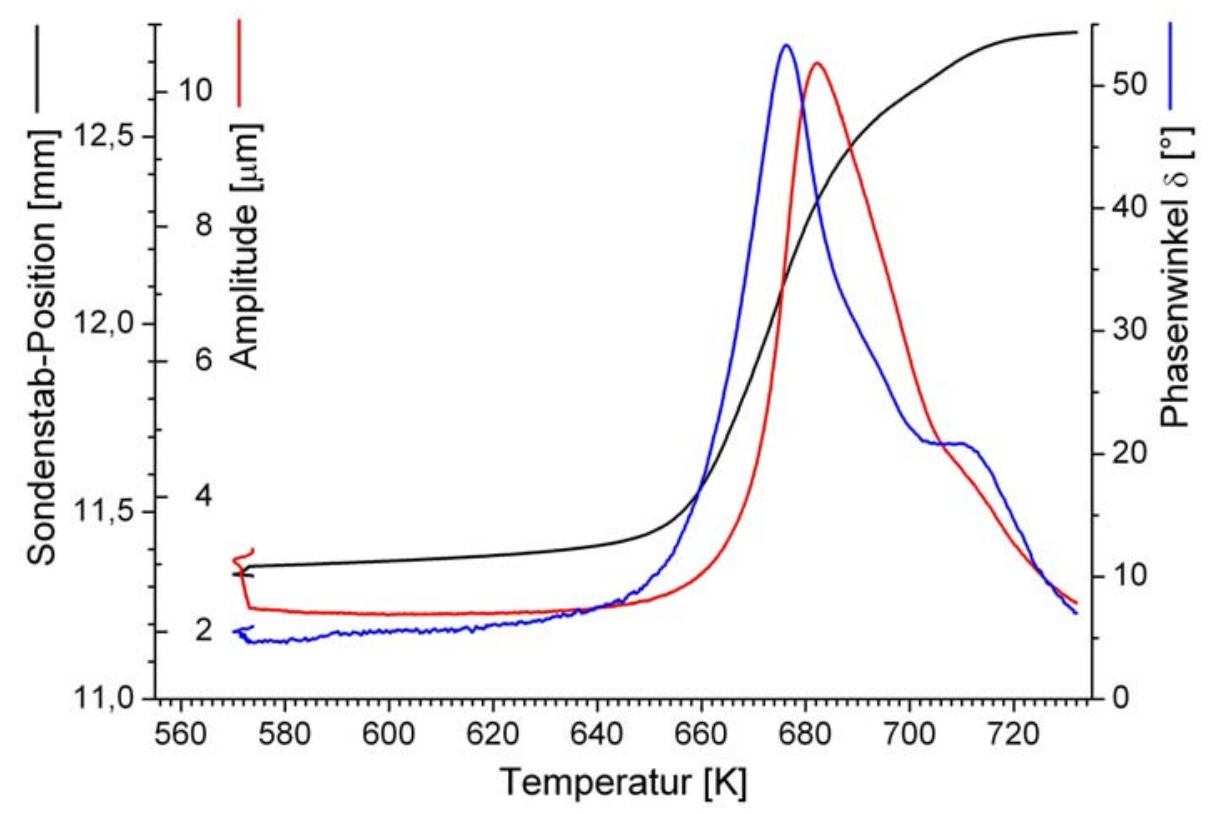

Abb. 4.4.1: Rohdaten einer DMA-Messung an PdCuSi bei $1 \mathrm{~Hz}$ und $5 \mathrm{~K} / \mathrm{min}$, Achsenbeschriftungen farbkodiert

Die Auftragung aller drei Messgrößen erfolgte dabei zur direkten Vergleichbarkeit in nur einer Auftragung. Anstelle einer Legende sind die Achsenbeschriftungen farbkodiert. Die Son- 
denstab-Position bezeichnet damit die Höhe des Sondenstabes über der Bodenplatte, deren Nullpunkt so kalibriert wurde, dass sie der aktuellen Probenlänge, also dem Abstand der Klemmbacken entspricht. Sie zeigt zunächst einen nahezu linearen Verlauf, steigt oberhalb $640 \mathrm{~K}$ mit einem Onset bei 657(3) K stark an und durchläuft einen Wendepunkt bei 673(1) K. Danach nimmt die Änderung der Sondenstabposition und damit der Probenlänge wieder ab und stagniert oberhalb $730 \mathrm{~K}$.

Die Amplitude, in Rot aufgetragen, ist gleich der durch das Anlegen der dynamischen Kraft von $200 \mathrm{mN}$ erreichten Auslenkungsamplitude. Nach Anfangseffekten wird ein nahezu konstanter Verlauf beobachtet, auf den ebenfalls ein starker Anstieg zu einem Maximum folgt. Der Onset liegt bei 668(4) K, der erste Wendepunkt bei 676,3(8) K und das Maximum bei 682,3(1) K. Der Wendepunkt auf der fallenden Flanke ist schwer zuzuordnen, zusätzlich ist einen Knick bei 705(5) K zu sehen.

Der Phasenwinkel zwischen Anregung und Auslenkung beschreibt eine ähnliche Kurve, die zu tieferen Temperaturen verschoben ist. Er besitzt einen Onset bei 659(8) K zu einem Maximum bei 676,3(1) K. Der Verlauf des Phasenwinkels besitzt auf der Tieftemperaturflanke des Maximums einen Wendepunkt bei 670(2) K und auf der Hochtemperaturflanke zwei Knickpunkte bei 684(2) K und 701(2) K, denen zwei darauf folgende, verschmierte Maxima bei 692(2) K und 711(2) K zugeordnet werden können.

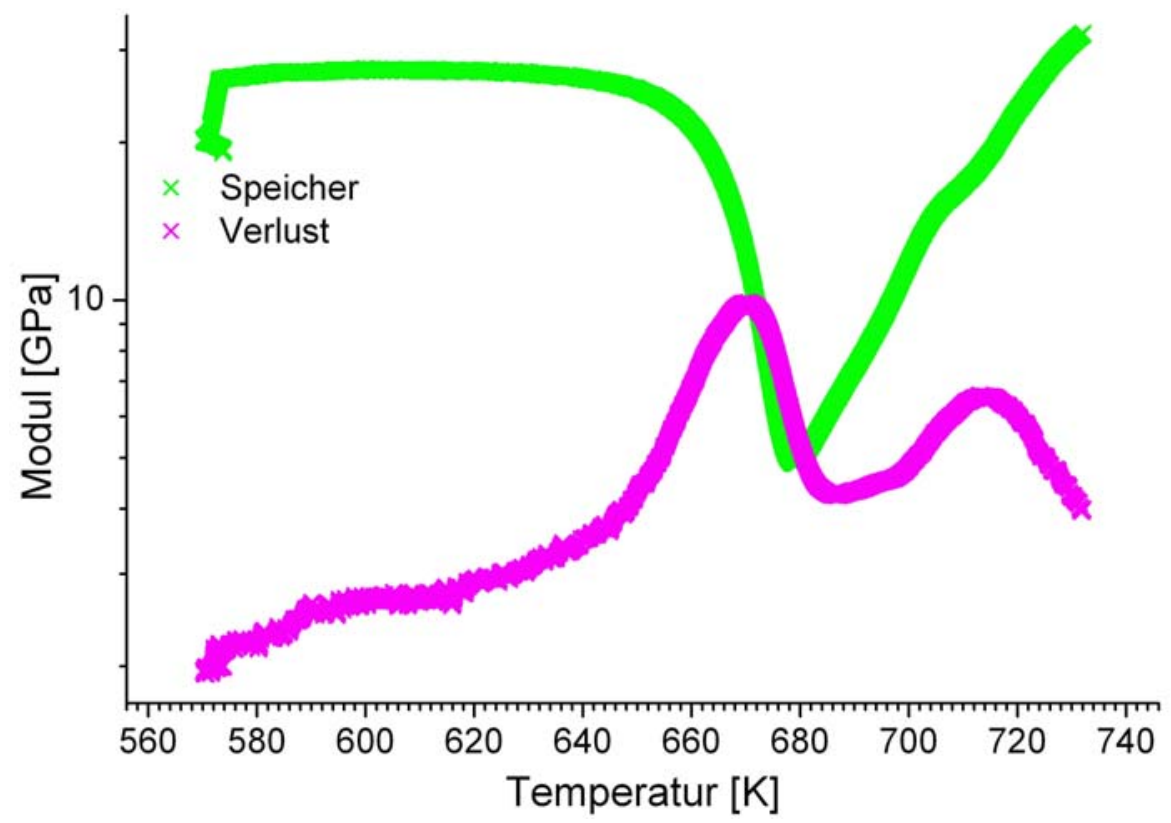

Abb. 4.4.2: Verlauf von Speicher- und Verlustmodul einer DMA-Messung bei $1 \mathrm{~Hz}$ und $5 \mathrm{~K} / \mathrm{min}$

Die Verläufe vom Speichermoduls E' und vom Verlustmoduls E', die dem Real- und Imaginärteil des Elastizitäts- oder Youngs Modul E* entsprechen, lassen sich mit Kenntnis der Abmessungen von Länge $l=11,3 \mathrm{~mm}$, Breite $b=1,3 \mathrm{~mm}$ und Dicke $d=28 \mu \mathrm{m}$, wie in Kapitel 2.4 beschrieben, berechnen. Dabei wird berücksichtigt, dass sich bei einer Längenänderung auch die Querschnittsfläche verändert. Das Probenvolumen wird dabei als konstant angenommen, was einer Poisson-Zahl von $v=0,5$ entspricht (vergleiche Kapitel 2.4). Verlängert sich also die Probe, so verringert sich die Querschnittsfläche, was bei konstanten Kräften eine 
Erhöhung der Spannung $\sigma$ bedeutet. Der Elastizitätsmodul ist nicht direkt spannungsabhängig, sein Temperaturverlauf dagegen schon, wie am Ende dieses Kapitels behandelt. Den Verlauf von Speicher- und Verlustmodul zeigt Abbildung 4.4.2. Dabei ist der Zehnerlogarithmus der Moduln als Ordinate aufgetragen, um beide Verläufe in einer Auftragung besser vergleichbar darzustellen. Um Verwirrungen zu vermeiden sei darauf hingewiesen, dass dies das Erkennen der Wendepunkte der Kurven in nicht logarithmierter Darstellung, die im Folgenden angegeben sind, erschwert.

Nach einem anfänglichen Anstieg in $E$ ' und $E$ ' 'während der Haltezeit von 4 min, steigt $E$ ' seicht weiter an zu einem Maximum bei 606(5) K. $E$ ', besitzt in diesem Bereich einen Wendepunkt, der fast ein Sattelpunkt ist bei 605(4) K. Darauf folgt ein Abfall mit verschmiertem Onset von 657(10) in $E$ ', begleitet von einem Anstieg in $E^{\prime}$,' der zunächst einen leichten Knick bei 613(5) K, einen Onset bei 651(5) K und einen Wendepunkt bei 661(3) K zeigt. Das Maximum von $E$ ', liegt bei 670(1) K und fällt mit dem Wendepunkt in $E$ ' bei 671(2) K zusammen. E' erreicht sein Minimum bei 679(1) K kurz oberhalb der Temperatur von 677(2) K, bei der $E$ ', einen Wendepunkt zeigt. $E$ ', erreicht ein Minimum bei 687(2) K, gefolgt von einem nahezu linearen Anstieg bis zu einem Knick bei 699(2) K. E' steigt in diesem Bereich oberhalb 679(1) K kontinuierlich an. Dieser Anstieg scheint aber ein niedriges Maximum bei 704(4) K zu überdecken, worauf ein Wendepunkt bei 722(5) K folgt. Dies zeigt sich in $E$ ', in einem Wendepunkt bei 705(2) $\mathrm{K}$ und einem Maximum bei 714(1) $\mathrm{K}$.

Besonders festzuhalten bleibt, dass beide Verlustmaxima auf ihrer Tieftemperaturflanke asymmetrisch verbreitert sind und dass der Speichermodul zunächst langsam und dann rasch abfällt, bevor er in einem zumindest zweistufigen Prozess wieder ansteigt.

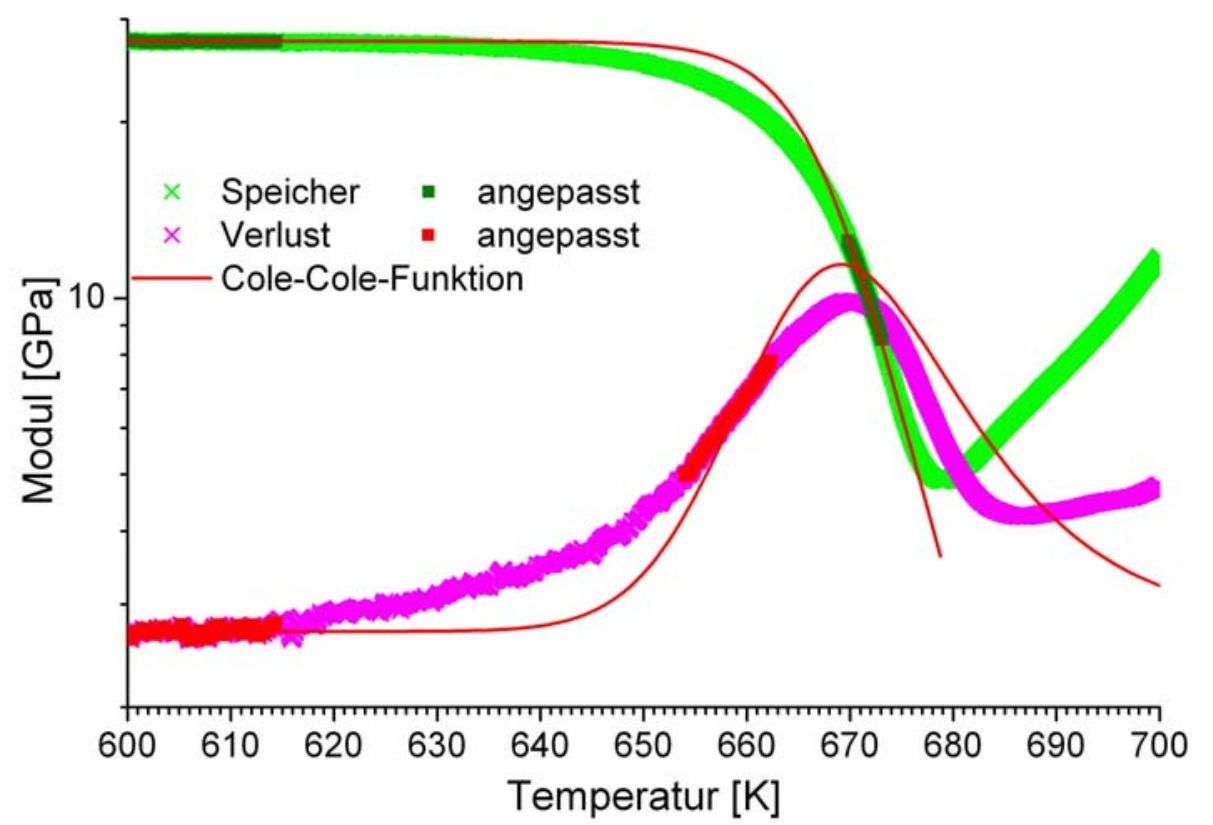

Abb. 4.4.3: Cole-Cole-Fit einer DMA-Messung an PdCuSi bei $1 \mathrm{~Hz}$ und $5 \mathrm{~K} / \mathrm{min}$

Eine Ausschnittsvergrößerung des Abfalls des Speichermoduls im Bereich 600 - $700 \mathrm{~K}$ zeigt Abbildung 4.4.3. An die rot und oliv hervorgehobenen Bereiche wurde eine Anpassung mit einer Cole-Cole-Fitfunktion durchgeführt. Dies entspricht einer Havriliak-Negami-Fitfunktion mit festem $\gamma(22,23)$. Zusätzlich wird zur besseren Anpassung bei Temperaturen unterhalb 
$615 \mathrm{~K}$ eine konstante Verschiebung $E_{N C L}$ angenommen (fester Offset). Die Relaxationsstärke $\Delta E=E_{\infty}-E_{0}$ wird gleich $E_{\infty}=E(T \rightarrow 0) \approx \Delta E$ gesetzt, da $E_{\infty}>>E_{0}$. Bei der Berechnung der am besten passenden Parameter ist zu beachten, dass der Abfall des Fits an $E$ ' nicht steiler als der gemessene Abfall von $E^{\prime}$ im Bereich des Wendepunkts ist. Die am besten zu beiden Messkurven, Speicher- und Verlustmodul, passenden Parameter zeigt Tabelle 4.4.1. Bereits auf den ersten Blick ist zu sehen, dass die Messdaten nicht in allen Bereichen mit den gefitteten Kurven zusammenfallen. Sowohl im Speicher- als auch im Verlustmodul weichen die Messdaten früher vom nahezu linearen Verlauf ab als die Fits. Der Onset des Abfalls des Fits an $E^{\prime}$ bei 660(5) K ist jedoch mit dem der Messdaten bei 657(10) K. Der gefittete Abfall ist aber weniger verschmiert. Sein Verlauf nähert sich dem der Messkurve an und schneidet diese bei $671(2) \mathrm{K}$ in deren Wendepunkt unter kleinem Winkel. Der Onset des Fits an $E$ "' bei 653(5) K ist ebenfalls nahezu gleich dem gemessenen Onset bei 651(5) K. Fit und Messwerte besitzen einen Schnittpunkt bei 659(2) K in der Nähe des Wendepunkts der Messkurve bei 661(3) K. Das Maximum des Fits an $E$ '” bei 669(1) K ist zu tieferen Temperaturen verschoben und höher als das gemessene. Oberhalb $675 \mathrm{~K}$ passen die Fits nicht mehr zu den gemessenen Daten. Alle Fehlerangaben beziehen sich hierbei auf die Unsicherheit in der geometrischen Auswertung der Fitfunktionen und stehen nicht in Relation zu den angegebenen Fehlern der Fitparameter in Tabelle 4.4.1.

Tab. 4.4.1: Parameter der Cole-Cole-Anpassung einer DMA-Messung an PdCuSi bei $1 \mathrm{~Hz}$ und $5 \mathrm{~K} / \mathrm{min}$

\begin{tabular}{|l|l|l|l|l|l|l|l|}
\hline Parameter & $\mathrm{f}$ & $\Delta \mathrm{E}$ & $E_{\mathrm{NCL}}$ & $\tau_{0}$ & $D_{\mathrm{VFT}}$ & $T_{\mathrm{VFT}}$ & \\
\hline Wert & $1 \mathrm{~Hz}$ & $27,513 \mathrm{GPa}$ & $2,695 \mathrm{GPa}$ & $10^{-12} \mathrm{~s}$ & 5,59 & $550 \mathrm{~K}$ & 0,72 \\
\hline Fehler & fix $^{*}$ & $0,004 \mathrm{GPa}$ & $0,003 \mathrm{GPa}$ & fix & 0,01 & $2 \mathrm{~K}$ & 0,02 \\
\hline
\end{tabular}

*: Werte wurden nicht angepasst, sondern als konstant vorausgesetzt

Eine verbesserte Anpassung der Messwerte im Bereich des Verlustmaximums kann erreicht werden, wenn für die Relaxationsstärke $\Delta E$ einen festen Wert von $24 \mathrm{GPa}$ eingesetzt wird (Abb. 4.4.4). Dies geschieht auf Kosten der Anpassung von $E$ ' unterhalb von $650 \mathrm{~K}$. Die verbleibenden angepassten Fitparameter zeigt Tabelle 4.4.2.

Oberhalb $650 \mathrm{~K}$ passt der so erhaltene Fit deutlich besser an den Verlauf des gemessenen Speichermoduls. Im Bereich des Wendepunkts ist der Verlauf des Fits geringfügig flacher als jener der Messwerte. Auch der gemessene Verlustmodul lässt sich im Bereich des Wendepunkts besser anpassen. Es ist dennoch eine Diskrepanz im Bereich zwischen dem Knick in $E$ "' bei $613(5) \mathrm{K}$ und dem gewichteten Bereich oberhalb $654 \mathrm{~K} \mathrm{zu}$ beobachten. Der Fit schneidet die Messkurve bei 658(1) K knapp unter deren Wendepunkt unter einem deutlich kleineren Winkel und auch die Höhe des Maximums wird besser angepasst. 


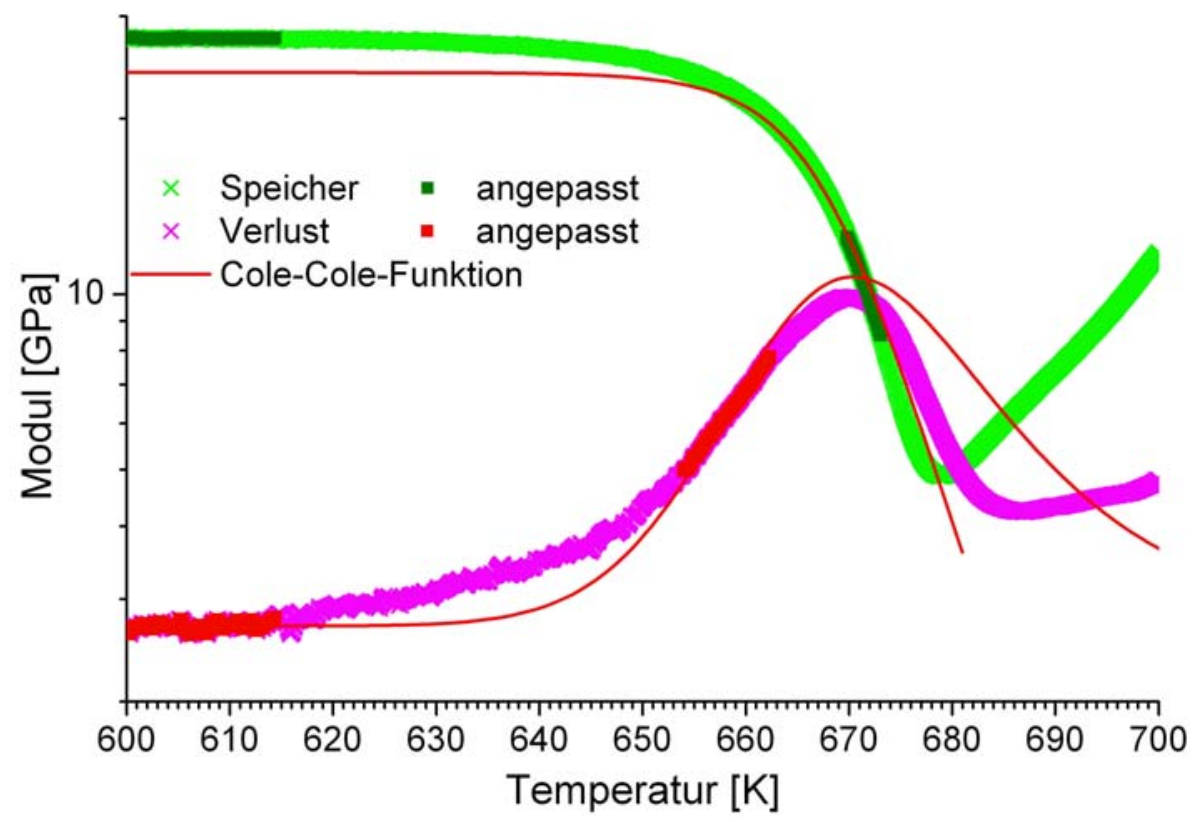

Abb. 4.4.4: Modifizierter Cole-Cole-Fit einer DMA-Messung an PdCuSi bei $1 \mathrm{~Hz}$ und $5 \mathrm{~K} / \mathrm{min}$

Tab. 4.4.2: Parameter der modifizierten Cole-Cole-Anpassung einer DMA-Messung an $\mathrm{PdCuSi}$ bei $1 \mathrm{~Hz}$ und $5 \mathrm{~K} / \mathrm{min}$

\begin{tabular}{|l|l|l|l|l|l|l|l|}
\hline Parameter & $\mathrm{f}$ & $\Delta \mathrm{E}$ & $E_{\mathrm{NCL}}$ & $\tau_{0}$ & $D_{\mathrm{VFT}}$ & $T_{\mathrm{VFT}}$ & \\
\hline Wert & $1 \mathrm{~Hz}$ & $24 \mathrm{GPa}$ & $2,695 \mathrm{GPa}$ & $10^{-12} \mathrm{~s}$ & 7,78 & $515 \mathrm{~K}$ & 0,75 \\
\hline Fehler & fix $^{*}$ & fix $^{*}$ & $0,003 \mathrm{GPa}$ & fix $^{*}$ & 0,02 & $5 \mathrm{~K}$ & 0,07 \\
\hline
\end{tabular}

*: Werte wurden nicht angepasst, sondern als konstant vorausgesetzt

\section{Einfluss der Messparameter: Raten-, Frequenz- und Kraftabhängigkeit}

Beim Vergleich zweier spektroskopischer DMA-Messungen sind, wie in Kapitel 2.3 beschrieben, alle der Versuchsparameter Heizrate, Frequenz und Spannung relevant für die Untersuchung der viskoelastischen Eigenschaften. Um ihren Einfluss im Detail zu untersuchen, bedarf es einer systematischen Variation dieser Versuchsbedingungen.

Deutlich sichtbar ist der Effekt der Variation der Heizrate im Tangens des Phasenwinkels $\delta$ der gleich dem Verhältnis von Verlust- zu Speichermodul ist (16). Abbildung 4.4.5 zeigt den Verlauf von $\tan \delta$ als Funktion der Temperatur für acht dynamische Messungen bei $1 \mathrm{~Hz}$ und Kräften von $100 \mathrm{mN}$ statisch und $50 \mathrm{mN}$ dynamisch bei Heizraten von $1 \mathrm{~K} / \mathrm{min}, 5 \mathrm{~K} / \mathrm{min}$, $20 \mathrm{~K} / \mathrm{min}$ und $40 \mathrm{~K} / \mathrm{min}$.

Die Verschiebung des Onsets des Maximums fasst Tabelle 4.4.3 zusammen. Soweit möglich, wurden Mittelwerte gebildet, der geschätzte Fehler liegt bei $2 \mathrm{~K}$. Es ist keine signifikante Verschiebung zwischen Heizraten von $1 \mathrm{~K} / \mathrm{min}$ und $5 \mathrm{~K} / \mathrm{min}$ zu beobachten. Der Anstieg der Onset-Temperatur mit steigender Heizrate ist aber von $5 \mathrm{~K} / \mathrm{min}$ zu $20 \mathrm{~K} / \mathrm{min}$ und zu $40 \mathrm{~K} / \mathrm{min}$ deutlich. Dabei ist darauf hinzuweisen, dass alle Messungen mit derselben Kalibrierung für Heizen mit $5 \mathrm{~K} / \mathrm{min}$ durchgeführt wurden, worauf in der Diskussion eingegangen wird. Die in 
[RAM94, S. 39] gemessenen Korrekturwerte sind in Tabelle 4.4.3 angegeben. Zusätzlich ist festzustellen, dass nur die Kurve bei $1 \mathrm{~K} / \mathrm{min}$ ein Maximum zeigt, während bei den anderen Messungen die Proben mit zunehmender Geschwindigkeit auseinander gezogen werden.

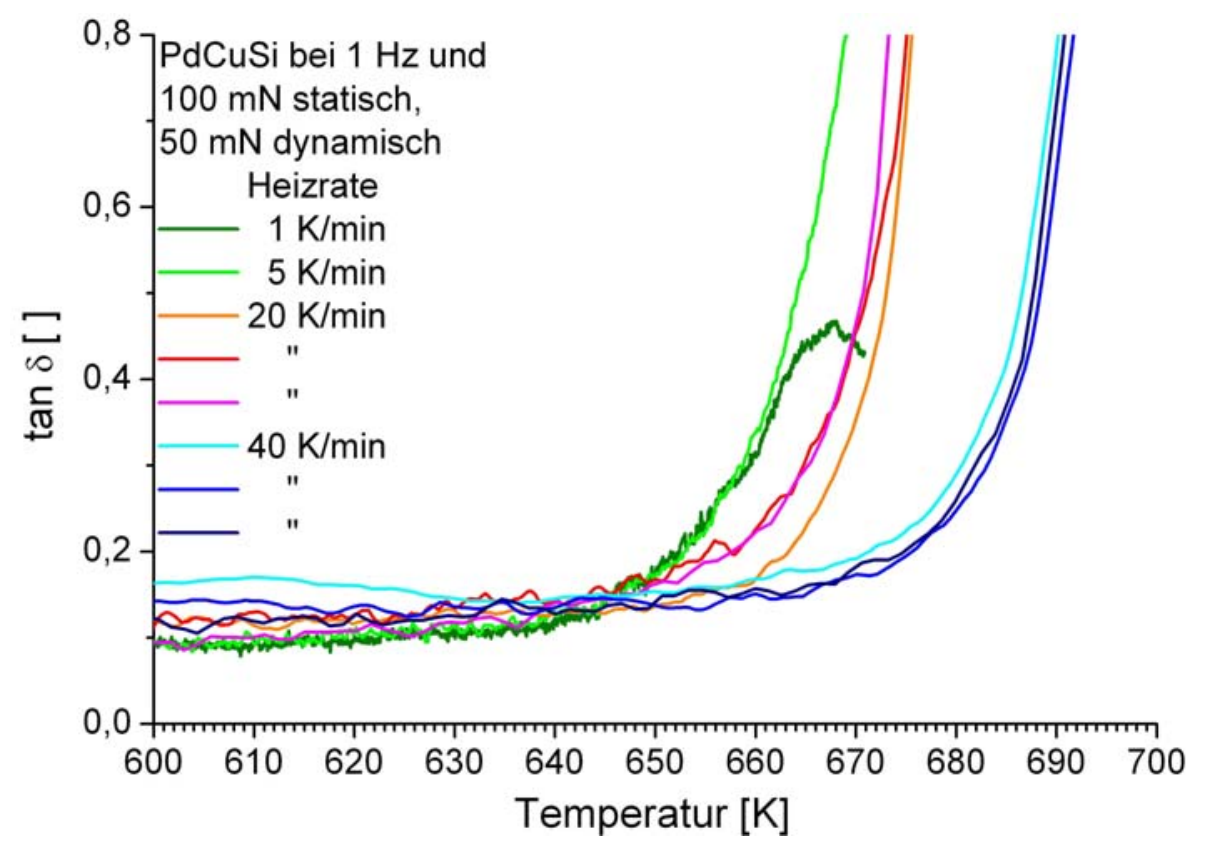

Abb. 4.4.5: Einfluss der Heizrate auf den Phasenwinkel bei der Spektroskopie an PdCuSi

Tab. 4.4.3: Heizratenabhängigkeit der Onsets der tan $\delta$-Maxima bei der Spektroskopie an PdCuSi mit Korrektur aus [RAM94]

\begin{tabular}{|l|l|l|l|l|}
\hline Heizrate $[\mathrm{K} / \mathrm{min}]$ & 1 & 5 & 20 & 40 \\
\hline Onset $[\mathrm{K}]$ & 654 & 655 & $671^{*}$ & $683^{*}$ \\
\hline Heizratenkorrektur $[\mathrm{K}]$ & 1,9 & 0 & $-4,5$ & $-13,8$ \\
\hline Korrigierter Onset [K] & 656 & 655 & 667 & 669 \\
\hline
\end{tabular}

*: Mittelwert dreier Messungen

Abbildung 4.4.6 zeigt die Auswirkung der Änderung von Frequenz und Amplitude auf den Tangens der Phasenwinkels $\delta$. Bei den Frequenzen $1 \mathrm{~Hz}$ und $10 \mathrm{~Hz}$ regelt der Messrechner die angegebene Sollamplitude durch Einstellung einer passenden dynamischen Kraft ein. Die statische Kraft beträgt dabei $120 \%$ der dynamischen und wird somit auch geregelt. Im Fall einer Frequenz von $10 \mathrm{~Hz}$ und einer Sollamplitude von $6 \mu \mathrm{m}$ reicht die zur Verfügung stehende Kraft erst ab $652 \mathrm{~K}$ aus, um die Sollamplitude zu erreichen. Dies führt zu einem Sprung im Kurvenverlauf. Bei tieferen Temperaturen liegt die Amplitude im Bereich zwischen 5,5 $\mu \mathrm{m}$ und 6,0 $\mu \mathrm{m}$. Bei niedrigeren Frequenzen als $1 \mathrm{~Hz}$ versagt die Regelung der Amplitude, weshalb ein festes Kräfteverhältnis von $200 \mathrm{mN}$ zu $100 \mathrm{mN}$ bzw. von $400 \mathrm{mN}$ zu $200 \mathrm{mN}$ gewählt wird. Dies resultiert in Amplituden von etwa $2 \mu \mathrm{m}$ bzw. $3 \mu \mathrm{m}$ bei $600 \mathrm{~K}$. Bei $650 \mathrm{~K}$ beträgt die Amplitude bereits 2,5 $\mu \mathrm{m}$ bzw. 4,6 $\mu \mathrm{m}$. 
Die beobachtete Systematik ist in Tabelle 4.4.4 zusammengefasst: Eine Erhöhung der Frequenz bewirkt eine Verschiebung des Onsets des Anstiegs in Tangens von $\delta$ zu höheren Temperaturen. Je höher die eingeregelte Amplitude, desto steiler verläuft die Kurve, die Amplitudenabhängigkeit der Onsets ist dagegen nicht signifikant. Bei den mit * gekennzeichneten Onsets wird der Wendepunkt der Kurve vor dem Auseinanderziehen der Probe nicht erreicht, deshalb wird die Tangente nicht im Wendepunkt, sondern im letzten Wert angelegt. Dadurch wird eine zu kleine Steigung angenommen; die Werte stellen eine untere Abschätzung dar. Der geschätzte Fehler beträgt $4 \mathrm{~K}$.

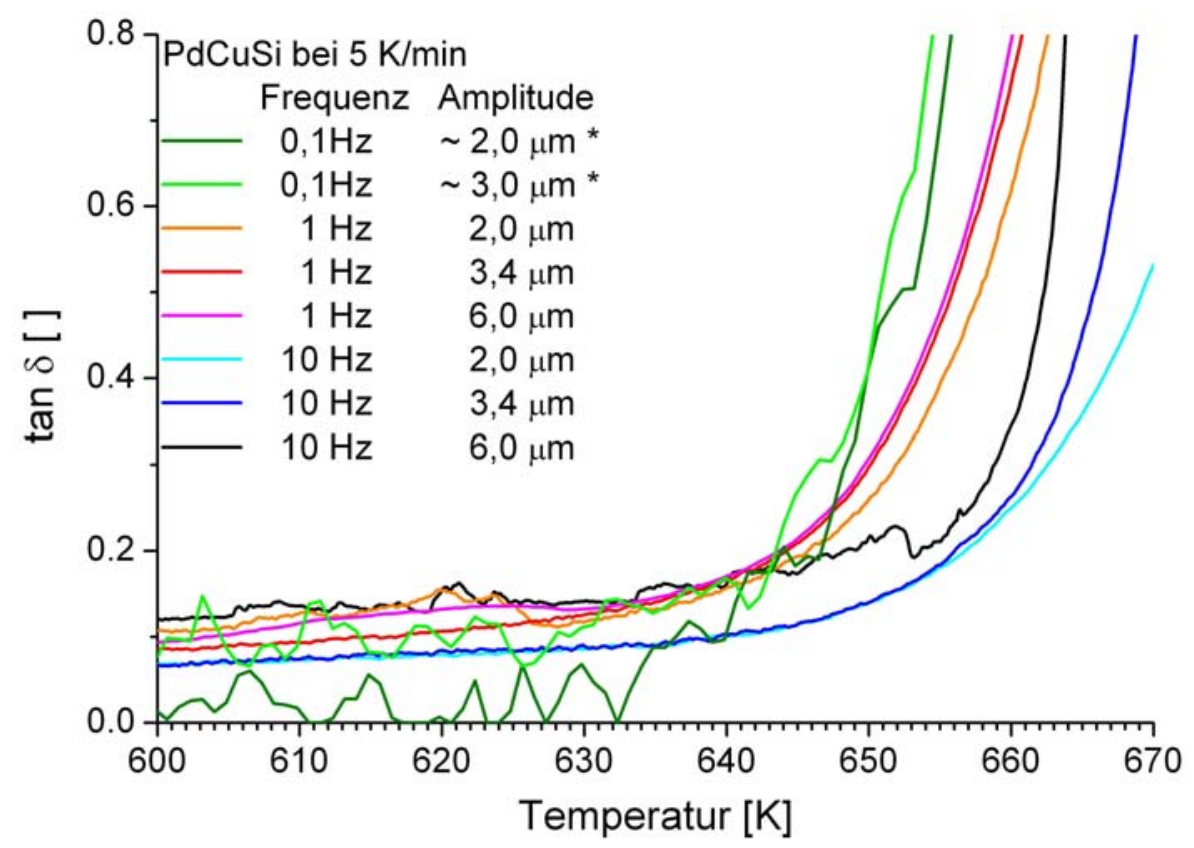

*: Messung bei konstanter Kraft, angegeben ist die Amplitude bei $600 \mathrm{~K}$

Abb. 4.4.6: Einfluss der Frequenz und der Amplitude auf den Phasenwinkel bei der Spektroskopie an $\mathrm{PdCuSi}$

Tab. 4.4.4: Einfluss der Frequenz und der Amplitude auf den Onset von tan $\delta$

\begin{tabular}{|l|l|l|}
\hline Frequenz $[\mathrm{Hz}]$ & Amplitude $[\mu \mathrm{m}]$ & $\tan \delta$ - Onset $[\mathrm{K}]$ \\
\hline 0,1 & $\sim 2$ & 650 \\
\hline 0,1 & $\sim 3$ & $649^{*}$ \\
\hline 1 & 2 & 657 \\
\hline 1 & 3,4 & 654 \\
\hline 1 & 6,0 & 656 \\
\hline 10 & 2,0 & 662 \\
\hline 10 & 3,4 & $668^{*}$ \\
\hline 10 & 6,0 & $662^{*}$ \\
\hline
\end{tabular}

*: Untere Grenze des Onsets 
Eine Variation der Amplitude oder der Kräfte beeinflusst nicht nur die dynamischen Messgrößen, sondern spiegelt sich auch in den viskoelastischen Eigenschaften wider, die durch den statischen Anteil der Kraft bestimmt werden. Wie einleitend erwähnt, muss die statische Kraft stets deutlich größer sein als die dynamische, da die Probe sonst nicht straff gezogen wird und es im Extremfall zur Beschädigung der Messapparatur kommt. Eine Änderung der statischen Kraft beeinflusst insbesondere die Viskosität $\eta$ (5). Deshalb wurden die Messungen anstelle bei konstanter Amplitude bei konstanter Kraft durchgeführt. Dies entspricht nahezu einer konstanten Spannung, da die maximale statische Dehnung in Abbildung 4.4.7 10\% und in Abbildung 4.4.8 aufgrund der kürzeren Verweilzeit bei $20 \mathrm{~K} / \mathrm{min}$ gerade einmal $4 \%$ beträgt. Da Einschnürungen bei weniger als $10 \%$ Längenänderung noch nicht auftreten sollten, liegt die Reduktion der Querschnittsfläche bei als konstant angenommenem Volumen (vgl. Kap. 2.4) und somit auch die Zunahme der Spannung in derselben Größenordnung. Bei der Berechnung der Viskosität ist darauf zu achten, dass die durch thermische Ausdehnung auftretende Längenänderung von Proben, Stahl-Klemmen und Quarz-Aufhängung abgezogen werden muss. Es fällt daher schwer, einen separaten Wärmeausdehnungskoeffizienten für PdCuSi anzugeben. Bei einer Probenlänge von 10,9 mm beträgt die lineare Wärmeausdehnung bei ca. $530 \mathrm{~K} 3,9 \cdot 10^{-7} \mathrm{~m} / \mathrm{K}$, wie in Abbildung 4.4 .9 gezeigt (Probe identisch zur Messung bei $100 \mathrm{mN}$ in Abb. 4.4.7). Alle Proben wurden zuvor nicht ausgelagert, und die Messungen beginnen bei $573 \mathrm{~K}$ oder darunter. Wiederum wurde dieselbe, auf $5 \mathrm{~K} / \mathrm{min}$ angepasste Kalibrierung verwendet, weswegen die angegebenen Temperaturen um 4,5 K zu hoch sind (vgl. Tab. 4.4.3).

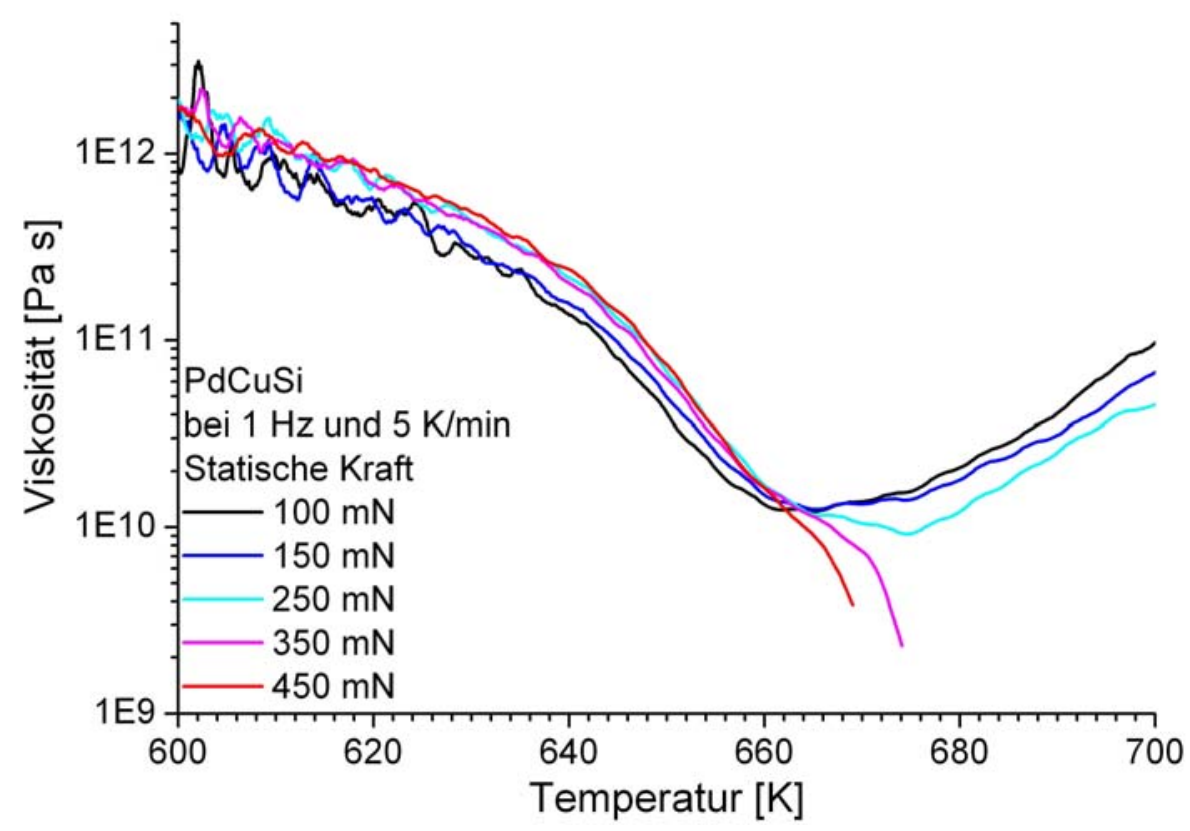

Abb. 4.4.7: Einfluss der Kraft auf die Viskosität bei der Spektroskopie an PdCuSi bei $1 \mathrm{~Hz}$ und $5 \mathrm{~K} / \mathrm{min}$

In Abbildung 4.4.7 fällt zunächst auf, dass die Viskosität nach einem Abfall bei den Messungen bei kleinen Kräften bis $250 \mathrm{mN}$ ein Minimum durchläuft und wieder ansteigt, während bei Kräften ab $350 \mathrm{mN}$ statischer Kraft die Probe auseinander fließt. Die Viskosität steigt systematisch mit der Kraft im Temperaturintervall zwischen $600 \mathrm{~K}$ und $663 \mathrm{~K}$, jener Temperatur, 
in deren Nähe sich die Kurven kreuzen. Danach verkehrt sich diese Systematik ins Gegenteil. Beim Heizen mit $20 \mathrm{~K} / \mathrm{min}$ fließen die Proben bei größeren Kräften vor Erreichen des interpolierten Schnittpunktes bei ca. $682 \mathrm{~K}$ auseinander. Unterhalb dieser Temperatur wird derselbe Zusammenhang beobachtet. Proben, die bei höherer statischer Kraft gemessen werden, besitzen eine größere Viskosität. Die drei Messungen bei $100 \mathrm{mN}$ sind in ihrem Viskositätsverlauf bis zu dieser Temperatur nahezu deckungsgleich. Bei $677 \mathrm{~K}$ schneiden sich die Viskositätsverläufe der Messungen bei $350 \mathrm{mN}$ und $450 \mathrm{mN}$ statischer Kraft.

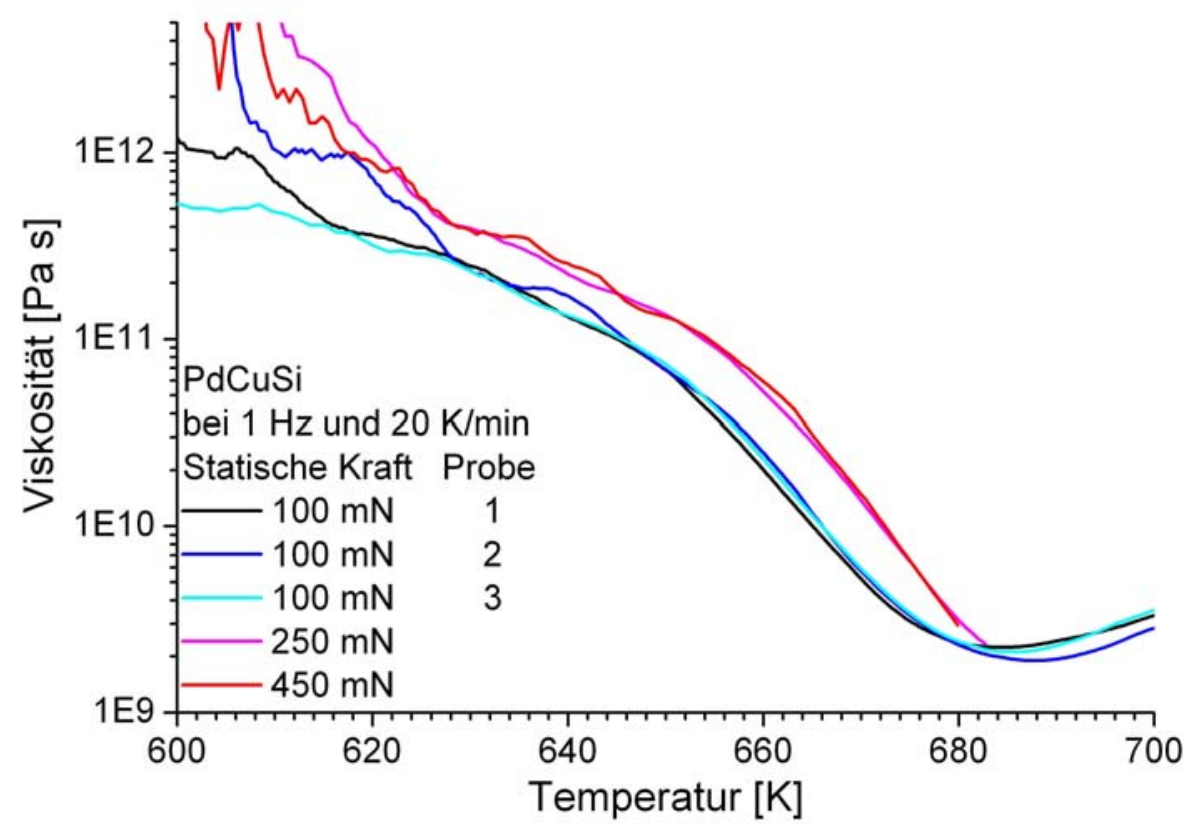

Abb. 4.4.8: Einfluss der Kraft auf die Viskosität bei der Spektroskopie an PdCuSi bei $1 \mathrm{~Hz}$ und $20 \mathrm{~K} / \mathrm{min}$

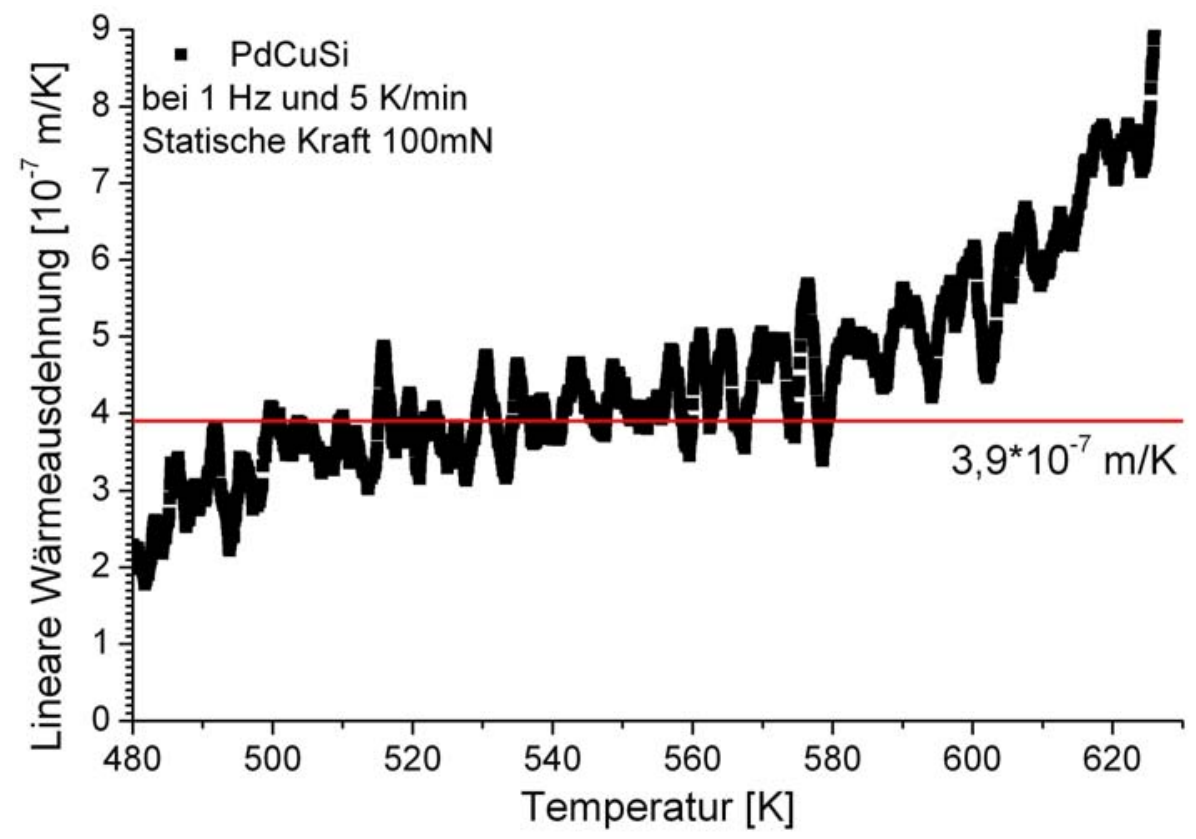

Abb. 4.4.9: Lineare Wärmeausdehnung von PdCuSi mit Stahl-Klemme und QuarzAufhängung 


\subsection{Dynamisch-mechanische Spektroskopie an ZrAlCu}

Zur besseren Vergleichbarkeit der Messungen an PdCuSi mit dem bereits untersuchten Verhalten von $\mathrm{ZrAlCu}$ bei 5,4 kHz (vgl. Kap. 2.5) werden auch in dieser Arbeit gemessene spektroskopische Untersuchungen an $\mathrm{ZrAlCu}$ betrachtet, allerdings bei $1 \mathrm{~Hz}$. Die Anpassung der Daten erfolgt dabei sowohl mit einer Cole-Davidson-Fitfunktion und einer Parameterwahl analog zu [RÖS04], als auch mit einer, wie in Kapitel 4.4 verwendeten, Cole-Cole-Fitfunktion.

Eine spektroskopische Messung an $\mathrm{ZrAlCu}$ bei konstanten Kräften von $200 \mathrm{mN}$ statisch und $150 \mathrm{mN}$ dynamisch, einer Heizrate von $3 \mathrm{~K} / \mathrm{min}$ und Frequenz von $1 \mathrm{~Hz}$ zeigt Abbildung 4.5.1. Die Anpassung an die rot und oliv hervorgehobenen Bereiche geschieht mit einer ColeDavidson-Fitfunktion, d. h. es wird beim Fitten mit der Havriliak-Negami-Funktion der Parameter $\alpha=1$ gesetzt und $\gamma$ variiert. Zusätzlich wurde $\tau_{\mathrm{o}}$ als freier Fitparameter zur Anpassung genutzt und dafür $T_{\mathrm{VFT}}$ und $D_{\mathrm{VFT}}$ als bekannt angenommen. Den am besten passenden Parametersatz zeigt Tabelle 4.5.1.

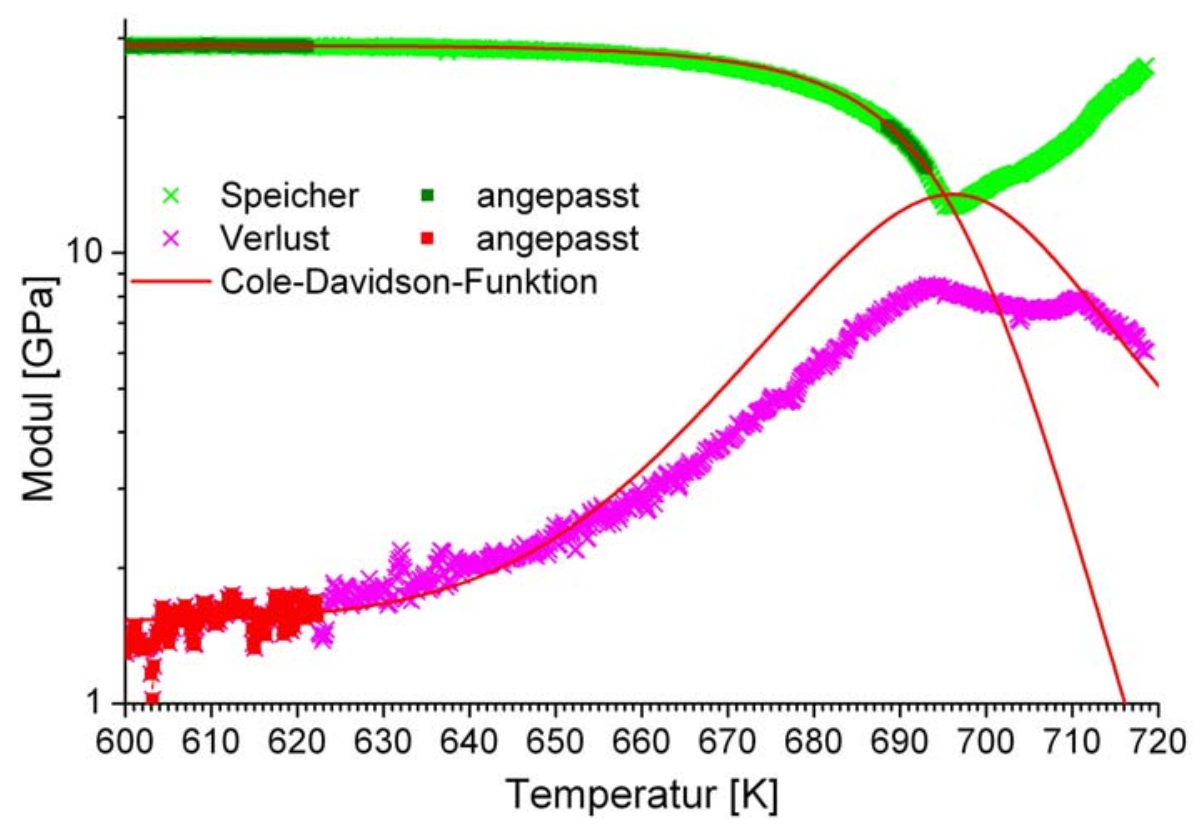

Abb. 4.5.1: Cole-Davidson-Fit einer DMA-Messung an $\mathrm{ZrAlCu}$ bei $1 \mathrm{~Hz}$ und $3 \mathrm{~K} / \mathrm{min}$

Tab. 4.5.1: Parameter der Cole-Davidson-Anpassung einer DMA-Messung an ZrAlCu bei $1 \mathrm{~Hz}$ und $3 \mathrm{~K} / \mathrm{min}$

\begin{tabular}{|l|l|l|l|l|l|l|l|}
\hline Parameter & $\mathrm{f}$ & $\Delta \mathrm{E}$ & $E_{\mathrm{NCL}}$ & $\tau_{0}$ & $D_{\mathrm{VFT}}$ & $T_{\mathrm{VFT}}$ & \\
\hline Wert & $1 \mathrm{~Hz}$ & $28,898 \mathrm{GPa}$ & $1,53 \mathrm{GPa}$ & $4,0 \cdot 10^{-14} \mathrm{~s}$ & 26,3 & $367 \mathrm{~K}$ & 0,67 \\
\hline Fehler & fix $^{*}$ & $0,002 \mathrm{GPa}$ & $0,02 \mathrm{GPa}$ & $3 \cdot 10^{-15} \mathrm{~s}$ & fix $^{*}$ & fix $^{*}$ & 0,023 \\
\hline
\end{tabular}

*: Werte wurden nicht angepasst, sondern als konstant vorausgesetzt

Wie auch im Fall von PdCuSi kann der Kurvenverlauf mit einem Parametersatz nicht zufrieden stellend angepasst werden. Insbesondere weist der Fit eine deutlich höhere Steigung auf, 
wohingegen der Verlauf des Speichermoduls im angepassten Bereich gut angenähert werden kann. Das Abknicken mit einem Onset bei ca. $680 \mathrm{~K}$ ist dabei in der gemessenen Kurve deutlich verschmierter, worüber die logarithmische Auftragung leicht hinwegtäuscht. Ein erstes Abweichen der Messkurve von einem horizontalen Verlauf lässt sich bereits bei $630 \mathrm{~K}$ erkennen. Bei $695 \mathrm{~K}$ knickt der Verlauf des Speichermoduls abrupt ab, ohne zuvor einen Wendepunkt zu durchlaufen. Der Anstieg des Verlustmoduls zu einem ersten Maximum bei $694 \mathrm{~K}$ ist sehr breit mit kontinuierlich wachsender Steigung.

Eine deutlich bessere Annäherung der Fitparameter an die Messung gelingt mit einer ColeCole-Fitfunktion ( $\alpha$ variabel, $\gamma=1$ ), wie gezeigt in Abbildung 4.5.2. Dabei werden analog zu Kapitel 4.4 $T_{\mathrm{VFT}}$ und $D_{\mathrm{VFT}}$ zum Anpassen genutzt und $\tau_{0}=10^{-12} \mathrm{~s}$ gewählt. Effektiv steht damit ein zusätzlicher, freier Parameter zur Verfügung. Den Parametersatz, der die rot und oliv gezeichneten Kurvenbereiche am besten beschreibt, zeigt Tabelle 4.5.2. Dabei wurden auch Werte des Verlauf des Verlustmoduls genutzt, da mit einer Cole-Cole-Anpassung ein deutlich niedrigeres Maximum im Fit erreicht werden kann. Die Steigung der Fitkurve ist nach wie vor zu steil, wodurch der Verlauf des Fits zu breit und zu hoch ausfällt. Etwas schlechter als in Abbildung 4.5.1 gelingt die Anpassung des Speichermoduls bei Temperaturen kurz vor dem Abknicken, wo der gemessene Speichermodul steiler abfällt als die angepasste Kurve.

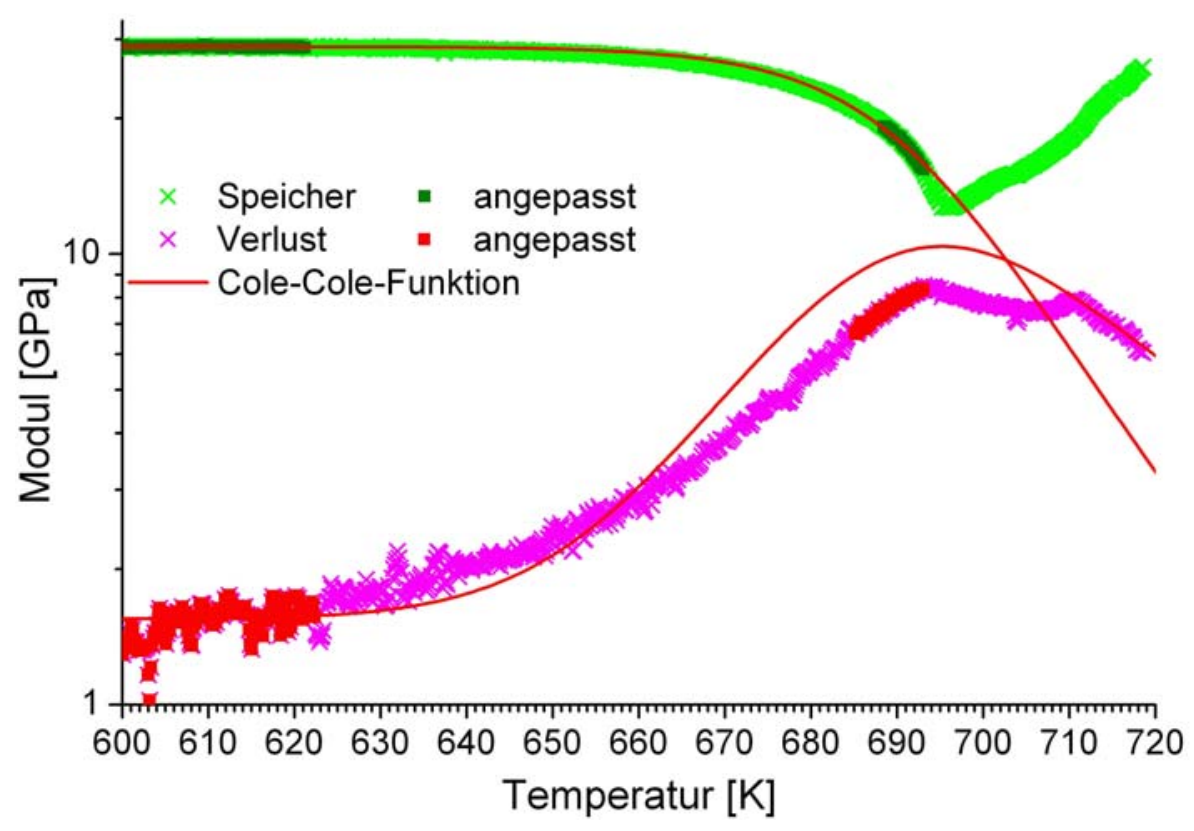

Abb. 4.5.2: Cole-Cole-Fit einer DMA-Messung an $\mathrm{ZrAlCu}$ bei $1 \mathrm{~Hz}$ und $3 \mathrm{~K} / \mathrm{min}$

Tab. 4.5.2: Parameter der Cole-Cole-Anpassung einer DMA-Messung an ZrAlCu bei $1 \mathrm{~Hz}$ und $3 \mathrm{~K} / \mathrm{min}$

\begin{tabular}{|l|l|l|l|l|l|l|l|}
\hline Parameter & $\mathrm{f}$ & $\Delta \mathrm{E}$ & $E_{\mathrm{NCL}}$ & $\tau_{0}$ & $D_{\mathrm{VFT}}$ & $T_{\mathrm{VFT}}$ & \\
\hline Wert & $1 \mathrm{~Hz}$ & $28,891 \mathrm{GPa}$ & $1,55 \mathrm{GPa}$ & $10^{-12} \mathrm{~s}$ & 15,7 & $432 \mathrm{~K}$ & 0,7 \\
\hline Fehler & fix $^{*}$ & $0,003 \mathrm{GPa}$ & $0,08 \mathrm{GPa}$ & fix & 1,1 & $13 \mathrm{~K}$ & 0,1 \\
\hline
\end{tabular}

*: Werte wurden nicht angepasst, sondern als konstant vorausgesetzt 
Eine direkte Gegenüberstellung zweier dynamischer Messungen an $\mathrm{ZrAlCu}$ und PdCuSi unter identischen Bedingungen in der Frequenz $1 \mathrm{~Hz}$, der Heizrate $3 \mathrm{~K} / \mathrm{min}$ sowie den Kräften von $200 \mathrm{mN}$ dynamisch und $150 \mathrm{mN}$ statisch zeigt Abbildung 4.5.3. Die zugehörigen Achsen in der Auftragung befinden sich links und unten für $\mathrm{ZrAlCu}$ und rechts und oben für PdCuSi. Um eine bessere Vergleichbarkeit zu erzielen, wurden die Abszissen um $48 \mathrm{~K}$ gegeneinander verschoben. Dies entspricht der Temperaturdifferenz im Auftreten der Verlustmaxima. Die logarithmisch aufgetragenen Ordinaten sind auf den Bereich von $5 \%$ bis $105 \%$ des jeweils maximalen Speichermoduls begrenzt.

Bei diesem direkten Vergleich sind deutliche Unterschiede zu beobachten, sowohl im Verlustals auch im Speichermodul der Messungen. Ein erstes Indiz für die Verschiedenheit zeigt sich schon während des Versuchs bei Temperaturen, in denen der Speichermodul abfällt: Am letzten in Abbildung 4.5.3 gezeigten Punkt der PdCuSi-Messung beträgt die statische Dehnung $11 \%$ bei $647 \mathrm{~K}$, danach fließt die Probe unkontrolliert auseinander. Dies beendet die Messung, während ZrAlCu oberhalb $695 \mathrm{~K}$ einen Knick im Speichermodul aufweist und wieder härter wird. Nach der Messung bis $727 \mathrm{~K}$ ist nur eine Gesamtdehnung von 5,4 \% erreicht, der Streifen ist aber so spröde, dass er sich nicht am Stück ausbauen lässt, sondern zerbricht. Während der Messung zeigt sich die im Vergleich zum ZrAlCu schnellere Veränderung der viskoelastischen Eigenschaften von PdCuSi besonders im Verlauf des logarithmisch aufgetragenen Verlustmoduls als Funktion der Temperatur: So besitzt PdCuSi bei ca. $625 \mathrm{~K}$ einen Onset zu einem Verlustmaximum, wohingegen das Verlustmodul von ZrAlCu kontinuierlicher ansteigt, ohne dass ein klarer Onset auf dieser Temperaturskala deutlich erkennbar wäre. Dasselbe Verhalten spiegelt sich auch in den Verläufen der Speichermoduln wider: Während $\mathrm{ZrAlCu}$ einen gestreckten Abfall aufweist, zeigt PdCuSi einen stärker gekrümmten Verlauf, der oberhalb $645 \mathrm{~K}$ rapide abfällt. Zu diesem Zeitpunkt beträgt die statische Dehnung 7,5 \%.

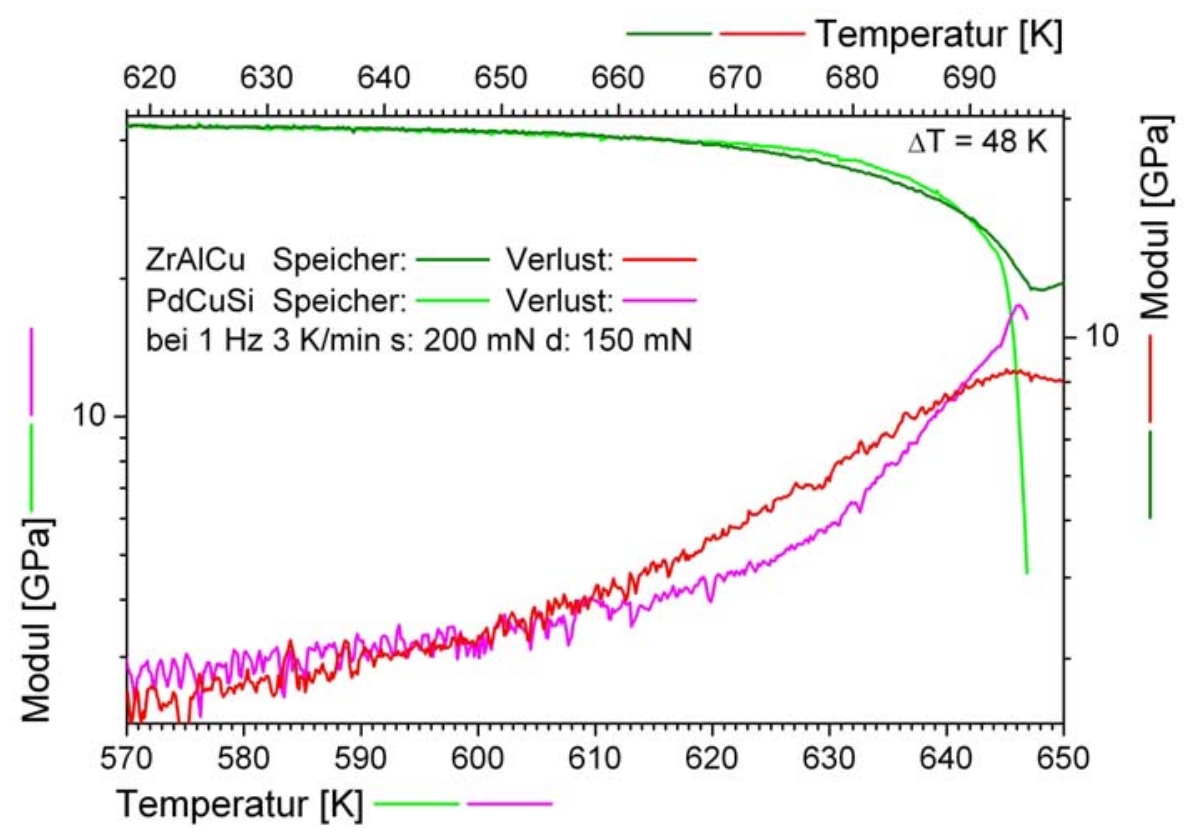

Abb. 4.5.3: Vergleich von dynamischen Messungen an $\mathrm{ZrAlCu}$ (Achsen: links \& unten) und $\mathrm{PdCuSi}$ (Achsen: rechts \& oben, um $48 \mathrm{~K}$ verschoben), die Ordinate ist jeweils auf den Bereich $5 \%-105 \%$ des maximalen Speichermoduls beschränkt. 


\subsection{Verhalten auf großen Temperaturskalen und Alterungsphäno- mene}

\section{Irreversible Alterungsphänomene beim ersten Aufheizen}

Die in Kapitel 4.5 gezeigten Messungen beschreiben den Temperaturbereich des Glasübergangs im Detail, nicht aber das mechanische Verhalten weit darunter. Um diesen Punkt geht es in diesem Kapitel, mit dem Ziel den Untergrund des Nearly Constant Loss (NCL, vgl. Kap. 2.5) besser vom Anstieg in den Verlustmessungen vor dem eigentlichen Maximum trennen zu können. Bei diesen Versuchen erfolgen die Messungen an der Auflösungsgrenze der DMA, und es zeigt sich, dass die mechanischen Eigenschaften stark von der thermischen und mechanischen Vorgeschichte der Proben abhängen. Deshalb werden Ausgangsproben, die nicht zuvor Heizzyklen unterworfen wurden, wohl definiert in Bezug auf die Frequenz, die Kräfte und die Heizraten, von tiefen, durch Flüssigstickstoffkühlung erreichten Temperaturen beginnend, spektroskopiert und mit Proben verglichen, die bereits nach festem Muster temperaturbehandelt wurden.

Den Vergleich der elastischen Moduln beim ersten und zweiten Aufheizen von einer PdCuSiund einer $\mathrm{ZrAlCu}$-Probe zeigen die Abbildungen 4.6.1-2. Alle Messungen sind bei $1 \mathrm{~Hz}$ und Kräften von $200 \mathrm{mN}$ statischer und $150 \mathrm{mN}$ dynamischer Kraft durchgeführt. Das erste Aufheizen der PdCuSi-Probe in Abbildung 4.6.1 erfolgt dabei zunächst mit $20 \mathrm{~K} / \mathrm{min}$ bis $573 \mathrm{~K}$, gefolgt von einem langsameren Heizen bei $3 \mathrm{~K} / \mathrm{min}$ bis $627 \mathrm{~K}$, wo bereits ein deutlicher Anstieg des Verlustmoduls auf etwa $8 \mathrm{GPa}$ beobachtet wird. Zur Temperaturkorrektur wird dabei eine, wie in Kapitel 3.10 beschriebene, Kalibrierung bei $3 \mathrm{~K} / \mathrm{min}$ verwendet, da ein besonderes Augenmerk auf der erreichten Endtemperatur liegt. Das anschließende Abkühlen erfolgt mit anliegenden Kräften, so schnell es die DMA zulässt. Die erreichte Kühlrate liegt bei ca. $30 \mathrm{~K} / \mathrm{min}$ bis etwa $450 \mathrm{~K}$ und verlangsamt sich darunter zunehmend. Das zweite Aufheizen erfolgt bei $20 \mathrm{~K} / \mathrm{min}$ und entsprechender Kalibrierung bis zum Auseinanderfließen der Probe bei ca. 646 K. Die Dehnung beträgt $3 \%$ im Punkt mit der höchsten, in Abbildung 4.6.1 gezeigten Temperatur. Die Messung an $\mathrm{ZrAlCu}$ erfolgt gleichermaßen, allerdings entspricht die Heizrate $3 \mathrm{~K} / \mathrm{min}$ beim ersten Aufheizen erst ab $583 \mathrm{~K}$ und wird bis $665 \mathrm{~K}$ geheizt. Das zweite Aufheizen wird nicht durch das Auseinanderfließen der Probe beendet, sondern erfolgt bis $940 \mathrm{~K}$. Bei $720 \mathrm{~K}$ beträgt die statische Dehnung 3,2\%.

Beim Vergleich der Veränderungen der mechanischen Eigenschaften der beiden Gläser durch eine Temperaturbehandlung sind drei wesentliche Unterschiede $\mathrm{zu}$ beobachten. Zum ersten sind beim zweiten Aufheizen die Speichermoduln beider Gläser relativ zum ersten Aufheizen zu höheren Werten verschoben. Der Verlauf des Speichermoduls von PdCuSi ist beim ersten Aufheizen dabei annähernd konstant, während das Speichermodul von ZrAlCu bereits bei ca. $420 \mathrm{~K} \mathrm{zu}$ steigen beginnt. Ab $600 \mathrm{~K}$ hat letzteres den Verlauf des zweiten Aufheizens annähernd erreicht; oberhalb $640 \mathrm{~K}$ gleicht der Verlauf des Speichermoduls während dem ersten Aufheizen dem des zweiten. Zweitens geht bei ZrAlCu mit dem Anstieg des Verlustmoduls beim ersten Aufheizen bis auf etwa 4 GPa ein Abfall des Speichermoduls einher. Dies tritt beim ersten Aufheizen von PdCuSi auf einen doppelt so hohen Wert des Verlusts nicht ein. Es ist eher sogar ein leichter Anstieg oberhalb $609 \mathrm{~K} \mathrm{zu}$ beobachten. Drittens gleicht der Verlauf des Verlustmoduls von PdCuSi beim ersten Aufheizen dem des zweiten bis etwa $580 \mathrm{~K}$ und liegt darüber leicht oberhalb. Ganz im Gegensatz dazu zeigt der Verlust bei ZrAlCu bis ca. 
$500 \mathrm{~K}$ keinen gemeinsamen Verlauf, vielmehr ist vage ein breites Maximum des deutlich höheren Verlusts beim zweiten Aufheizen bei ca. $350 \mathrm{~K}$ zu erkennen. Oberhalb $550 \mathrm{~K}$ ist wiederum der Verlust während des ersten Aufheizens höher als während des zweiten.

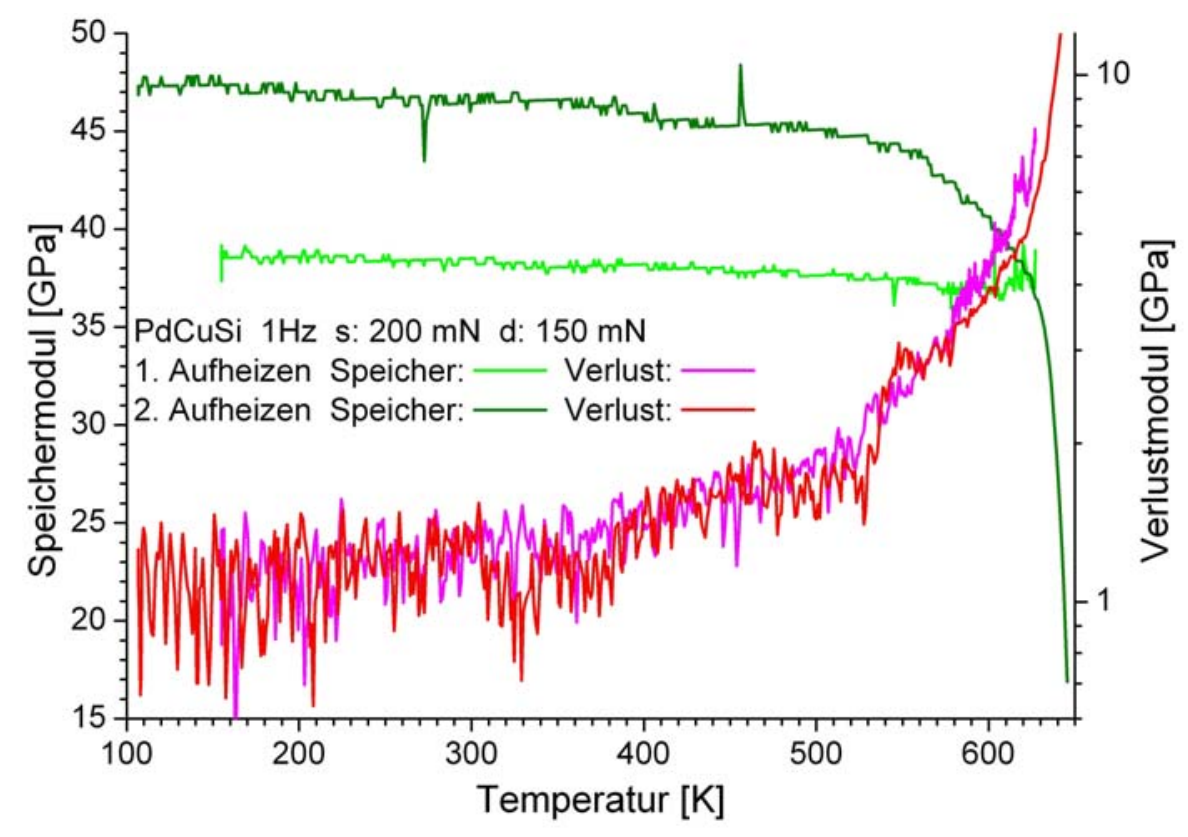

Abb. 4.6.1: Effekt der thermischen und mechanischen Vorbehandlung auf die Elastizitätsmoduln von $\mathrm{PdCuSi}$

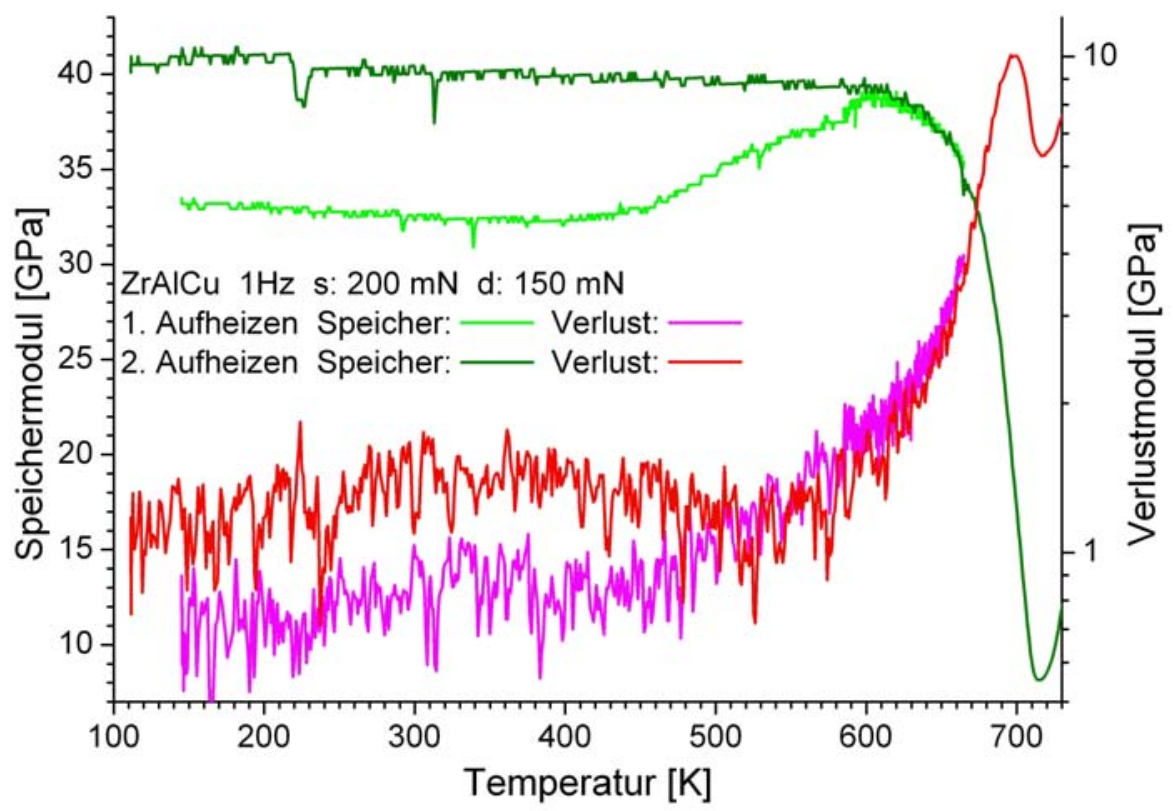

Abb. 4.6.2: Effekt der thermischen und mechanischen Vorbehandlung auf die Elastizitätsmoduln von $\mathrm{ZrAlCu}$

Um auch bei höheren Temperaturen im Bereich des Maximums den Einfluss einer vorangegangenen thermischen und mechanischen Vorbehandlung zu untersuchen, werden Referenzmessungen an Ausgangsproben herangezogen. Das Temperaturprogramm der in Abbildung 4.6.3 gezeigten Messungen entspricht dabei dem des ersten Aufheizens in den vorangegange- 
nen Untersuchungen. Verglichen werden der Verlauf des Verlustmoduls von Messungen an $\mathrm{ZrAlCu}$ bei einer Heizrate von $3 \mathrm{~K} / \mathrm{min}$ oberhalb von $585 \mathrm{~K}$ und $20 \mathrm{~K} / \mathrm{min}$ darunter (nicht gezeigter Bereich) und an PdCuSi, wo der Wechsel der Heizrate bereits bei $555 \mathrm{~K}$ erfolgt. Bei diesem Wechsel erfolgt bei allen Messungen eine einminütige Haltephase im Temperaturprogramm, wodurch ein Überschießen der Temperatur beim Heizratenwechsel reduziert wird.

Die erste Beobachtung während der Messung ist wiederum, dass die PdCuSi-Proben bei diesen Versuchsbedingungen auseinander fließen, während der Speichermodul (nicht gezeigt) der ZrAlCu-Proben ein Minimum durchläuft. Zur vereinfachten Darstellung wurden die Abszisse der Messkurven der PdCuSi-Proben in Abbildung 4.6.3 um 48 K gegen die der ZrAl$\mathrm{Cu}$-Proben verschoben, was der Differenz zwischen den Maxima der ZrAlCu-Kurvenverläufe und der Temperatur des Auseinanderfließens der vorbehandelten PdCuSi-Probe entspricht. Deutlich sichtbar ist, dass der Verlauf des Verlusts der PdCuSi-Messungen deutlich stärker gekrümmt ist als jener der ZrAlCu-Messungen. Während praktisch kein Unterschied in den Verläufen der ZrAlCu-Ausgangsproben und der vorbehandelten $\mathrm{ZrAlCu}$-Probe zu erkennen ist, gleichen sich die Verläufe der wie hergestellten und der vorbehandelten PdCuSi-Probe in ihrer Form, nicht aber in der Position. Durch eine Verschiebung in der Temperatur um 1,7 K und im Verlust um 0,7 GPa können beide Kurven in gezeigten Bereich zur Deckung gebracht werden.

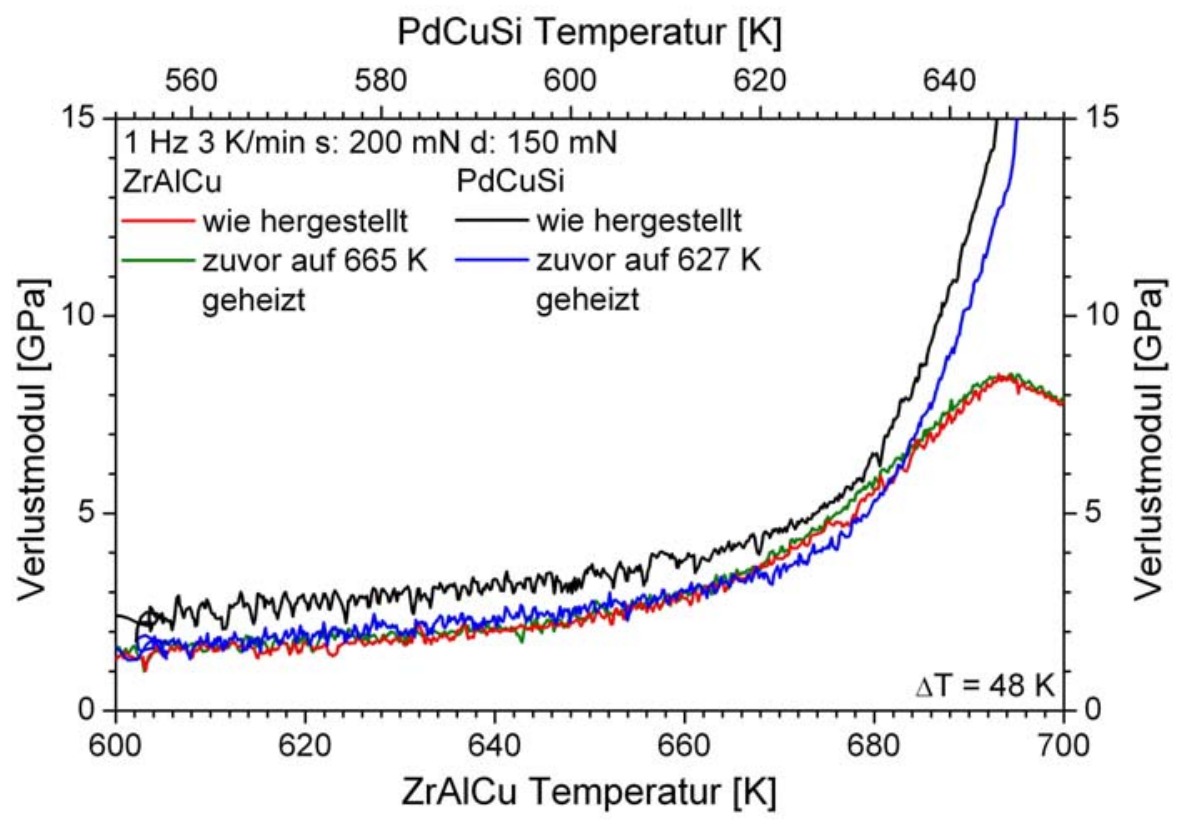

Abb. 4.6.3: Effekt der thermischen und mechanischen Vorbehandlung auf die Verlustmoduln von $\mathrm{ZrAlCu}$ und PdCuSi, verglichen mit Referenzmessungen an Ausgangsproben bei $3 \mathrm{~K} / \mathrm{min}$ Abbildung 4.6.4 zeigt eine zur vorangegangenen Abbildung analoge Messung, bei der die Heizrate konstant $20 \mathrm{~K} / \mathrm{min}$ beträgt und keine Halteperioden eingelegt werden, unter Verwendung einer entsprechenden Kalibrierung. Während die schnellere Heizrate den Effekt des Vorbehandelns auf die PdCuSi-Proben nicht verändert, ist hier einen deutlichen Unterschied zwischen den Kurvenverläufen des Verlusts der ZrAlCu-Ausgangsproben und der vorbehandelten $\mathrm{ZrAlCu}$-Proben zu erkennen: Im Vergleich zur Ausgangsprobe ist der Kurvenverlauf der vorbehandelten Probe auf der Tieftemperaturflanke des Maximums deutlich steiler und 
der Übergang zum Anstieg klarer ausgeprägt. Die Position des Maximums verschiebt sich um $3 \mathrm{~K} \mathrm{zu}$ höheren Temperaturen und es wird ein um 1,3 GPa höherer Wert erreicht.

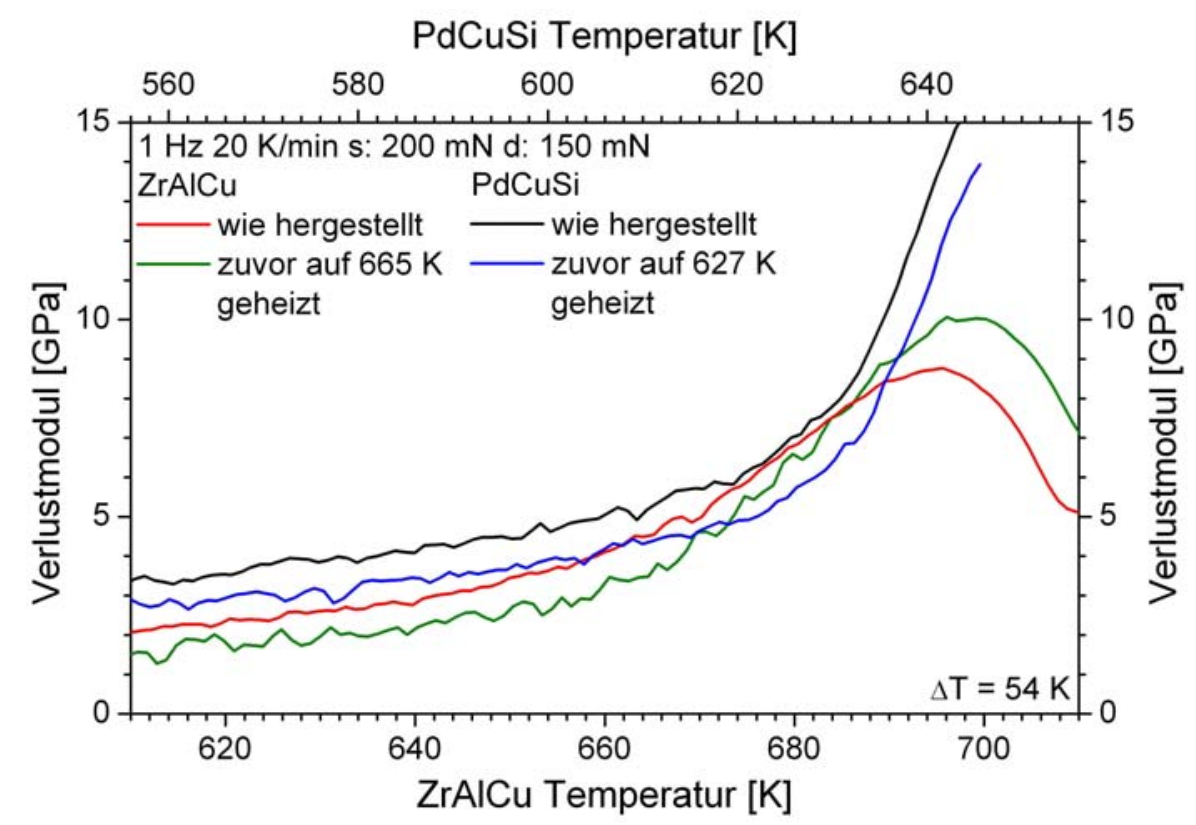

Abb. 4.6.4: Effekt der thermischen und mechanischen Vorbehandlung auf die Verlustmoduln von $\mathrm{ZrAlCu}$ und $\mathrm{PdCuSi}$, verglichen mit Referenzmessungen an Ausgangsproben bei $20 \mathrm{~K} / \mathrm{min}$

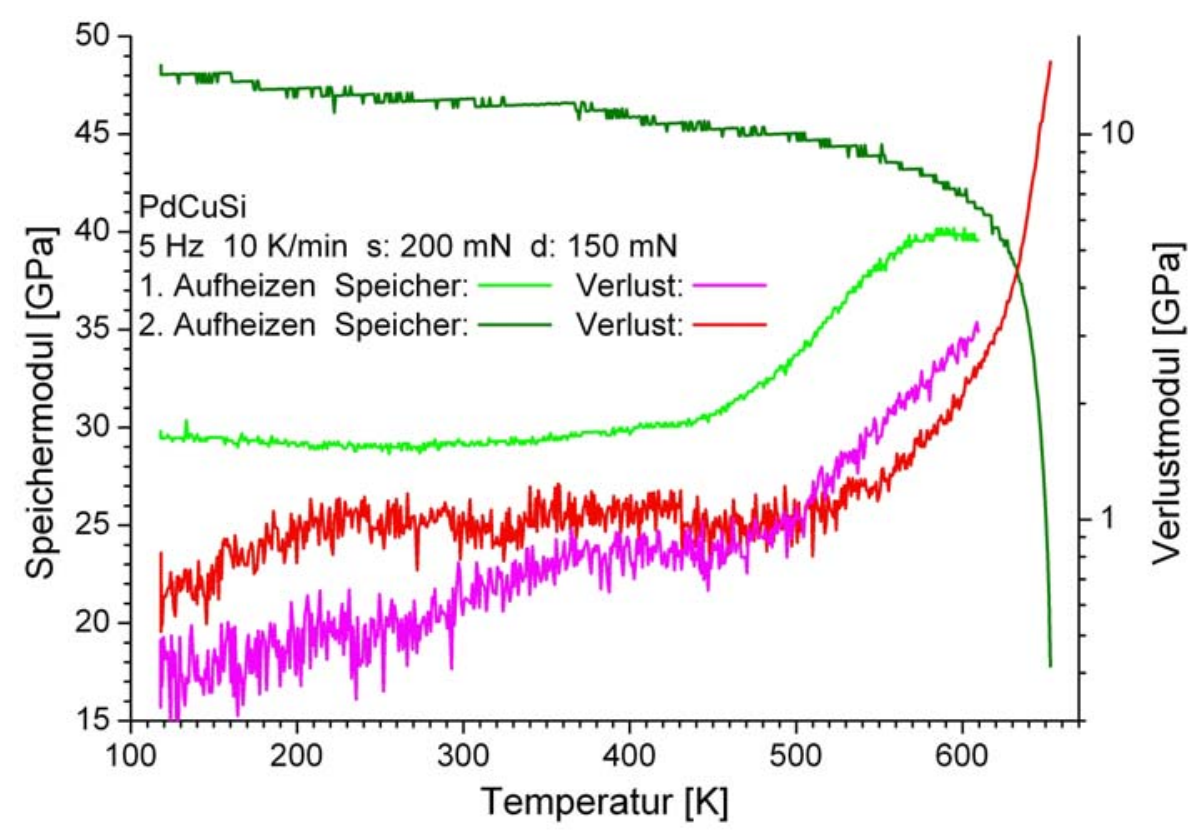

Abb. 4.6.5: Effekt der thermischen und mechanischen Vorbehandlung auf die Elastizitätsmoduln von PdCuSi bei einer höheren Frequenz von $5 \mathrm{~Hz}$

Bei höherer Frequenz von $5 \mathrm{~Hz}$, mittlerer Heizrate von $10 \mathrm{~K} / \mathrm{min}$ und gleichen Kräften zeigt auch PdCuSi in Abbildung 4.6.5 einen deutlichen Unterschied zwischen dem ersten und zweiten Aufheizen. Es ist auch einen Anstieg im Speichermodul während des ersten Aufheizens bis $609 \mathrm{~K}$ und anschließendem Kühlen bei anfangs ca. $30 \mathrm{~K} / \mathrm{min}$ mit anliegenden Kräften zu 
sehen. Die verwendete Kalibrierung zur Temperaturkorrektur wird durch Interpolation der Eichungen bei $3 \mathrm{~K} / \mathrm{min}$ und $20 \mathrm{~K} / \mathrm{min}$ gewonnen.

Es fällt auf, dass der Verlauf der elastischen Moduln in Abbildung 4.6.5 von der Kurvenform her mehr der von $\mathrm{ZrAlCu}$ bei $1 \mathrm{~Hz}$ und $20 \mathrm{~K} / \mathrm{min}$ bzw. $3 \mathrm{~K} / \mathrm{min}$ entspricht, als dem von PdCuSi unter diesen Bedingungen (vergleiche Abbildungen 4.6.1f). Im Verlustmodul wird beim ersten Aufheizen ein Anstieg beobachtet, der scheinbar über zwei Stufen erfolgt und erst oberhalb ca. $500 \mathrm{~K}$ über dem des zweiten Aufheizens liegt. Diese beiden Stufen zeigen sich auch im Speichermodul, der bei ca. $274 \mathrm{~K}$ in einen Bereich mit höherer Steigung abknickt und dann deutlich steigt, mit einem Onset von etwa $460 \mathrm{~K}$. Der Verlustmodul während des zweiten Aufheizens steigt zunächst leicht und ist dann im Bereich zwischen $220 \mathrm{~K}$ und $510 \mathrm{~K}$ nahezu konstant. Da beim zweiten Aufheizen ein wesentlich härteres Material untersucht wird, zeigt der Speichermodul beim zweiten Aufheizen deutliche Zeichen für das erreichen der Genauigkeitsgrenze der DMA, da das digitale Rauschen der Amplitude zu sichtbaren Stufen im Speichermodul führt.

\section{Heizratenabhängigkeit des Alterungsprozesses}

Aufgrund der Feststellung, dass die Messparameter den Verlauf des Speichermoduls (vgl. Abb.4.6.1 mit 4.6.5) stark beeinflussen ist es interessant, diese Abhängigkeit genauer zu untersuchen. Dazu wurden heizratenabhängige Messungen an PdCuSi und $\mathrm{ZrAlCu}$ durchgeführt. Bei niedrigen Heizraten von bis zu 0,5 K/min ist die Verwendung der Glykolkühlung wegen der besseren Temperaturstabilität vorteilhaft. Zusätzlich kann so das Rauschen im Verlauf des Phasenwinkels reduziert werden. Um eine Vergleichbarkeit zu gewährleisten ist es nötig, für jede Heizrate eine Temperaturkalibrierung der DMA durchzuführen. Dies erfolgt hier durch lineare Interpolation der Messungen der Schmelzpunkte an Zinn und Zink bei den auch für die Messungen an den metallischen Gläsern verwendeten Heizraten. Die Temperaturkorrektur erfolgt im anschließenden Vergleich mit den Literaturwerten.

Alle Messungen an PdCuSi und $\mathrm{ZrAlCu}$ werden bei festen Kräften von $500 \mathrm{mN}$ statisch und $400 \mathrm{mN}$ dynamisch sowie einer Spektroskopiefrequenz von $5 \mathrm{~Hz}$ durchgeführt. Die verwendeten Heizraten liegen zwischen $0,5 \mathrm{~K} / \mathrm{min}$ und $20 \mathrm{~K} / \mathrm{min}$. Abbildungen $4.6 .6+7$ zeigen die Verläufe von Speichermodul und tan $\delta$ der am stärksten unterschiedlichen Messungen:

Bei PdCuSi mit einer Heizrate von $0,5 \mathrm{~K} / \mathrm{min}$ erfolgt ein Anstieg des Speichermoduls mit einem Onset bei 370(20) K und einem Wendepunkt bei 470(10) K. Der Anstieg des tan $\delta$ erfolgt in zwei Schritten mit Onset-Punkten bei 533(10) K und 655(15). Alle Werte werden mit Hilfe der Tangentenmethode bestimmt (vgl. Abb. 4.3.3). Bei $20 \mathrm{~K} / \mathrm{min}$ verschiebt sich der Anstieg im Speichermodul um mehr als $100 \mathrm{~K}$ zu höheren Werten mit einem Onset bei 521(50) K und einem Wendepunkt bei 586(6) K. Gleichzeitig ist er weniger ausgeprägt. Der Verlauf des $\tan \delta$ ändert sich nur geringfügig in Bezug auf den ersten Onset, der nun bei $548(20) \mathrm{K}$ liegt. Bei höheren Temperaturen zeigt der $\tan \delta$ einen flacheren Verlauf, dessen zweiter Onset bei 685(25) K in der vergrößerten Darstellung in Abbildung 4.6.6 nicht mehr zu erkennen ist.

$\mathrm{ZrAlCu}$ zeigt ein ähnliches Verhalten, mit dem Unterschied, dass der Anstieg über einen größeren Bereich erfolgt und auch stärker ausgeprägt ist als bei PdCuSi. Onset und Wendepunkt bei 393(30) K bzw. 483(14) K bei einer Heizrate von $0,5 \mathrm{~K} / \mathrm{min}$ verschieben sich fast genauso 
stark zu 521(50) K bzw. 586(6) K bei einer Heizrate von $20 \mathrm{~K} / \mathrm{min}$. Auch der Verlauf des tan d flacht sich beim Wechsel von $0,5 \mathrm{~K} / \mathrm{min} \mathrm{zu} 20 \mathrm{~K} / \mathrm{min}$ deutlich ab. Wie bereits beim Vergleich der Verläufe der Verlustmodule in Abbildungen 4.4.2f und 4.5.1f auffällt, ist die Identifizierung zweier Onsets bei $\mathrm{ZrAlCu}$ weit weniger ausgeprägt als bei PdCuSi. Die Variation der Heizrate führt hier zu einer geringen Verschiebung von 534(20) K und 650(20) K zu 553(20) K und 687(20) K.

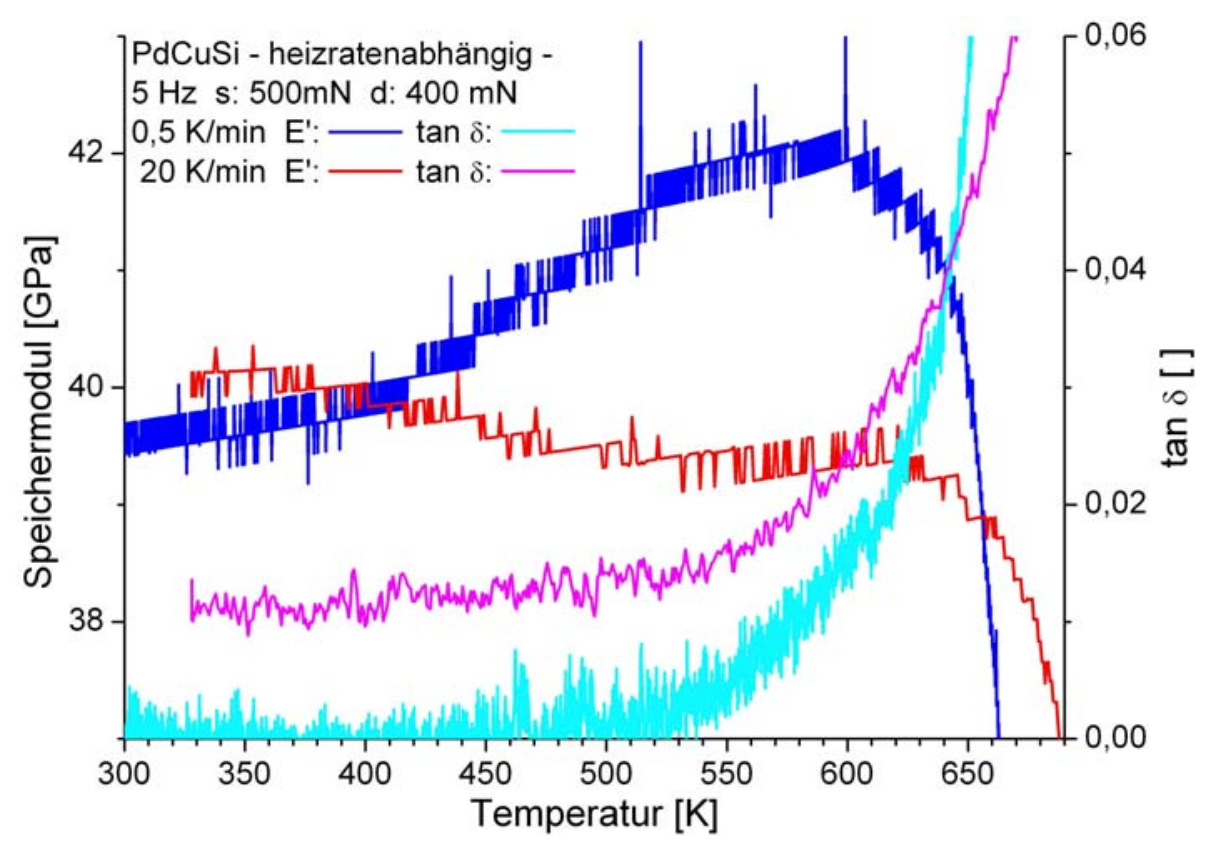

Abb. 4.6.6: Anstieg im Speichermodul von PdCuSi bei Heizraten von 0,5 K/min und $20 \mathrm{~K} / \mathrm{min}$ und Verlauf der zugehörigen $\tan \delta$

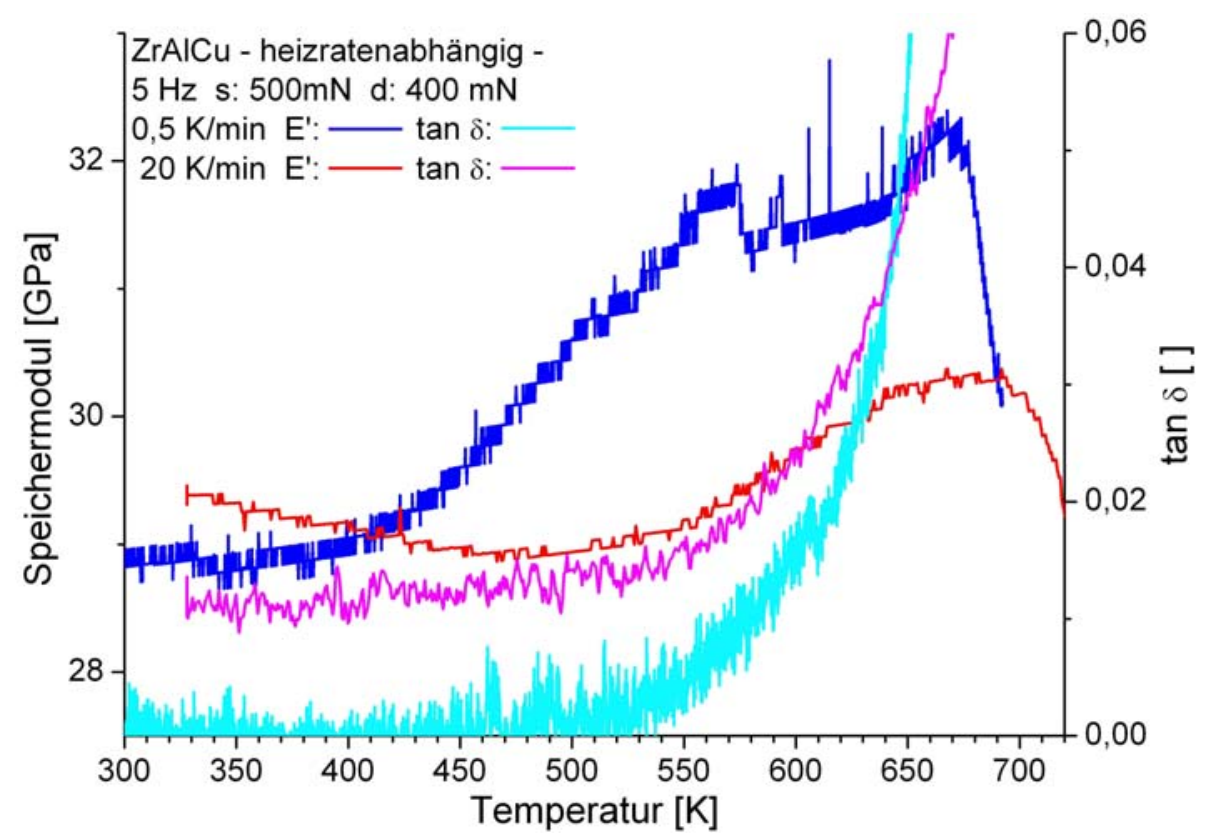

Abb. 4.6.7: Anstieg im Speichermodul von $\mathrm{ZrAlCu}$ bei Heizraten von 0,5 K/min und $20 \mathrm{~K} / \mathrm{min}$ und Verlauf der zugehörigen $\tan \delta$ 


\section{Untersuchungen an Polymeren}

\subsection{Probencharakterisierung}

Um die Abhängigkeit der makroskopischen mechanischen Eigenschaften und Relaxationen der Copolymere von deren mikro- bis mesoskopischen, durch die Synthese festgelegte, chemische Struktur erklären zu können, ist es notwendig, diese genau zu kennen und den Einfluss der Syntheseparameter zu untersuchen. Aus dieser Überlegung heraus werden für die Analyse jeweils eine Probe bei hohem und eine bei niedrigem Synthesedruck aus den hergestellten Serien gewählt. Innerhalb einer Serie wird der MAA-Fluss konstant gehalten, er unterscheidet sich aber stark von Serie 1 und 2 (unveränderter, mittlerer MAA-Fluss) zu Serie 3 (hoher MAA-Fluss) und zu Serie 12 (niedriger MAA-Fluss). Darüber hinaus sind die untersuchten Proben optisch klar und bei relativ konstanten Temperaturen und statischen Reaktionsbedingungen, ohne Auftreten einer Entmischung in der Reaktionskammer, synthetisiert.

\section{Spektroskopische Untersuchung der chemischen Zusammensetzung}

Die Bestimmung des MAA-Gehalts im Copolymer erfolgt mittels FTIR-, ATR-FTIR- und NMR-Spektroskopie, wie in Kapitel 3.8 beschrieben. Beispiele für die Leistungsfähigkeit und die Unterschiede dieser drei Methoden zeigen die folgenden Abbildungen 5.1.1-3.

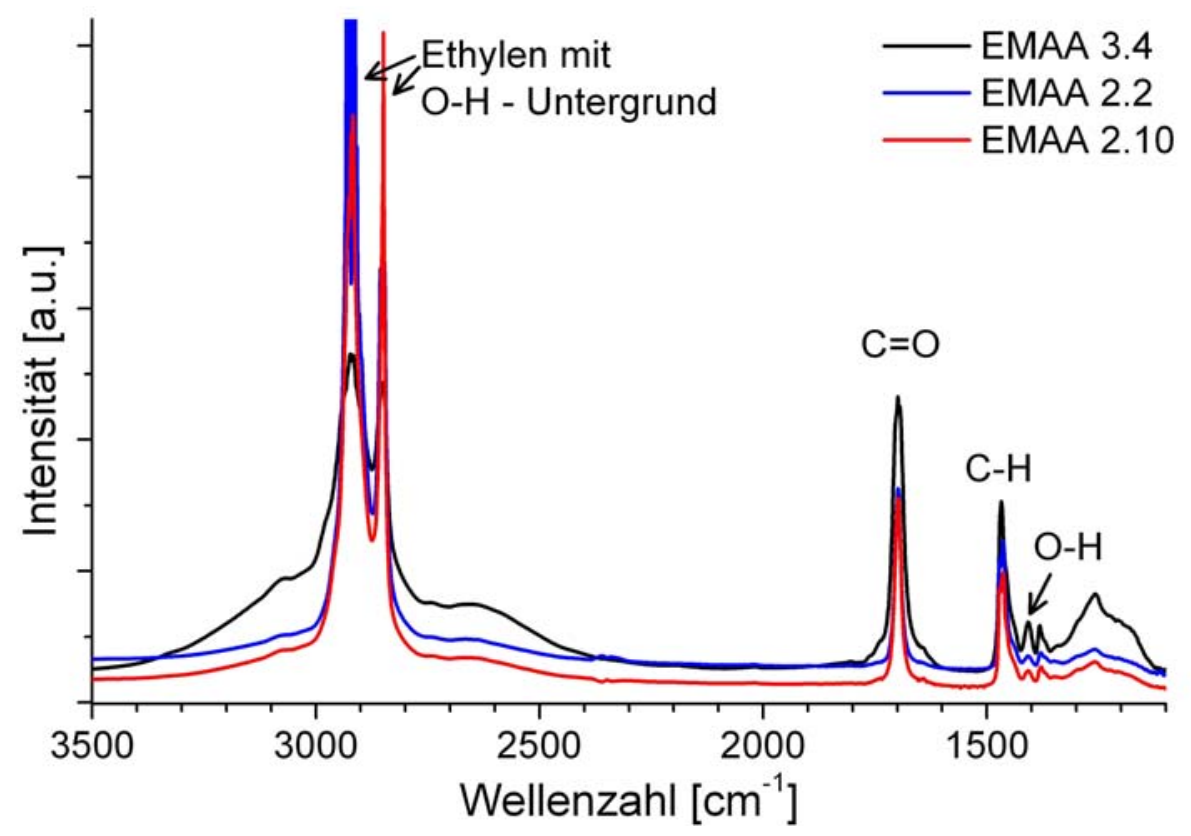

Abb. 5.1.1: FTIR-Spektrum in Absorption dreier EMAA-Folien mit stark unterschiedlichen Syntheseparametern

Die einfachste Möglichkeit der FTIR-Spektroskopie ist die Messung der frequenzabhängigen Absorption beim Durchstrahlen einer dünnen Folie. Um ein quantifizierbares Ergebnis zu erreichen, ist es wichtig, dass die Proben für eine ausreichende Transmission dünn genug und frei von Lufteinschlüssen sind. Abbildung 5.1.1 zeigt die Spektren dreier Proben, bei denen diese Voraussetzungen gegeben sind. EMAA 2.2 und 2.10 sind bei einer mittleren MAAFördermenge von $34,2 \mathrm{~g} / \mathrm{h}$ und Drücken von 1860 bar bzw. 1300 bar und EMAA 3.4 bei $116,0 \mathrm{~g} / \mathrm{h}$ und 2300 bar hergestellt. Die genauen Syntheseparameter zeigt die bereits in Kapi- 
tel 3.2 gezeigte Tabelle 5.1.2. Der unterschiedliche MAA-Fluss in Serie 2 und 3 führt zu deutlich sichtbaren Unterschieden im Spektrum, während die Änderung des Synthesedrucks keinen offensichtlichen Einfluss auf die FTIR-Spektren der beiden Proben der Serie 2 hat. Die relative Lage der Spektren ist stark von der Probenbeschaffenheit abhängig.

Die Berechnung des MAA-Gehalts geschieht durch den Vergleich der Flächen $A$ unter den gemessenen Absorptionsmaxima. Ihnen werden unterschiedliche Molekülschwingungen zugeordnet, welche für die Struktur der in das Polymer eingebauten Monomereinheiten charakteristisch sind (vergleiche Abb. 3.2.1). Die Ethylen-Maxima im Bereich $2800 \mathrm{~cm}^{-1}-3000 \mathrm{~cm}^{-}$ ${ }^{1}$ sind am stärksten ausgeprägt, können aber zur Quantifizierung nicht herangezogen werden, da in diesem Bereich auch ein breiter Untergrund durch O-H -Streckschwingungen vorliegt. Diese Quantifizierung geschieht, wie in [WITT99, BBD97] beschrieben, an den $\mathrm{C}=\mathrm{O}, \mathrm{C}-\mathrm{H}$ und O-H -Maxima im Bereich $1400 \mathrm{~cm}^{-1}-1800 \mathrm{~cm}^{-1}$ und dem darin bestimmten Parametersatz, der für einen MAA-Gehalt von 1,4 mol\% bis 9 mol\% bestimmt wurde. Der MAA-Gehalt $F_{M A A}$ lässt sich wie folgt berechnen:

$$
\frac{A_{C=O}}{A_{C-H}+A_{O-H}}=\frac{F_{M A A}}{a+b \cdot F_{M A A}}+c
$$

Tab. 5.1.1: Kalibration der quantitativen FTIR-Analyse nach [WITT99]

\begin{tabular}{|l|l|}
\hline Schwingung & Integrationsgrenzen \\
\hline $\mathrm{C}=\mathrm{O}$ & $1550 \mathrm{~cm}^{-1}-1950 \mathrm{~cm}^{-1}$ \\
\hline $\mathrm{C}-\mathrm{H}, \mathrm{O}-\mathrm{H}$ & $1435 \mathrm{~cm}^{-1}-1530 \mathrm{~cm}^{-1}$ \\
\hline
\end{tabular}

\begin{tabular}{|l|l|}
\hline Parameter & Wert \\
\hline a & 0,0272 \\
\hline b & 0,0474 \\
\hline c & 0,4117 \\
\hline
\end{tabular}

Diese Auswertungsmethode findet auch für die ATR-FTIR-Spektroskopie Anwendung, mit der die Proben untersucht worden sind, aus denen keine einschlussfreien, dünnen Folien gepresst werden konnten. Ein Beispiel für ein ATR-FTIR-Spektrum an zwei EMAA-Proben aus der bei hohem MAA-Fluss hergestellten Serie 3 zeigt die Abbildung 5.1.2. Der Synthesedruck beträgt bei Probe EMAA 3.4 2300 bar und bei EMAA 3.121300 bar. Die ATR-Spektren sind deutlich klarer als die Folien-Spektren. Die Absorptionsmaxima der beiden Proben mit unterschiedlichem Synthesedruck unterscheiden sich nicht signifikant.

Prinzipiell analog zu Gleichung (2), aber experimentell und physikalisch sehr verschieden zur Infrarotspektroskopie, ist auch die Bestimmung des MAA-Gehalts des Copolymers mittels NMR-Spektroskopie möglich. Abbildung 5.1.3 zeigt ein NMR-Spektrum derselben Proben aus Serie 3. Zur Berechnung des MAA-Gehalts wird auch hier die Fläche unter den zur MAA-Gruppe gehörenden ${ }^{13} \mathrm{C}$-Maxima in Relation zur Fläche unter allen ${ }^{13} \mathrm{C}$-Maxima gesetzt. Auch in Abbildung 5.13 sind kaum Unterschiede in den Spektren der Proben aus einer Serie bei unterschiedlichem Synthesedruck zu erkennen. Die Anwendbarkeit einer mehrdimensionalen NMR-Untersuchung ist Gegenstand andauernder Untersuchungen [BS07]. 


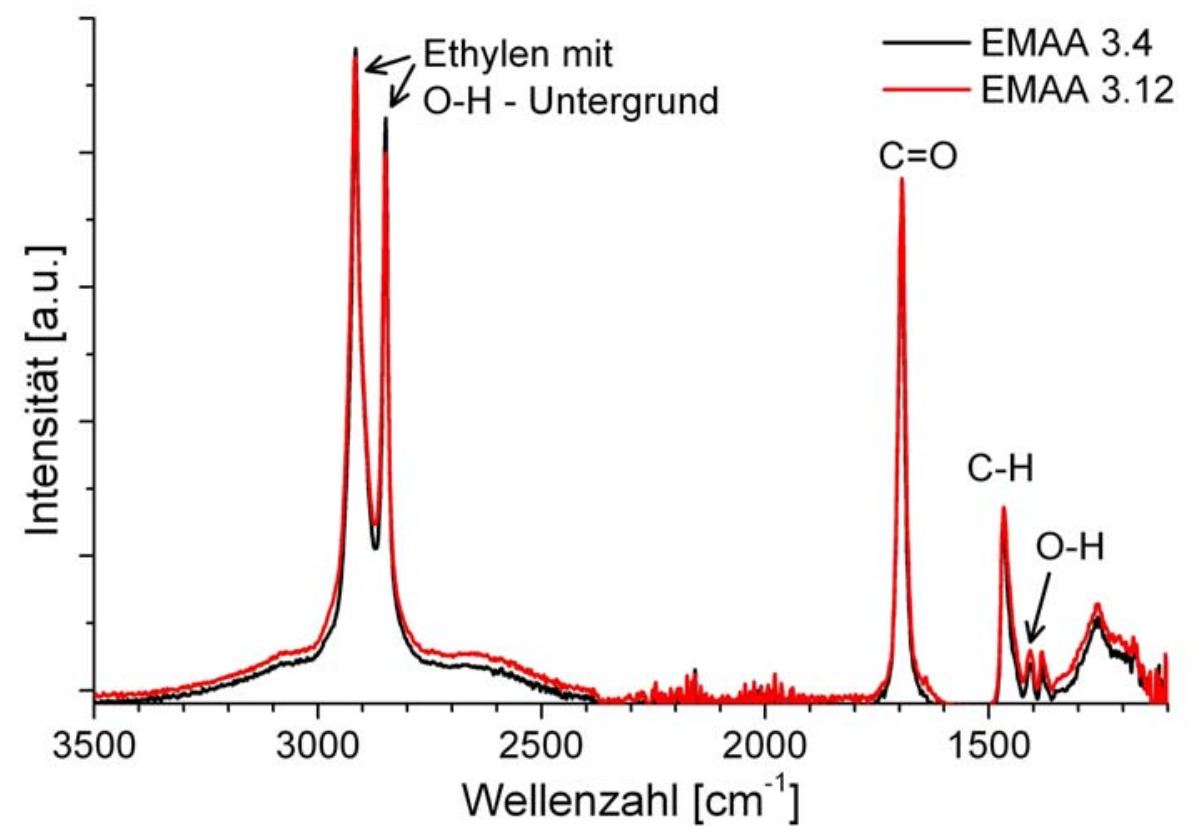

Abb. 5.1.2: ATR-FTIR-Spektrum zweier EMAA-Proben mit hohem MAA-Gehalt

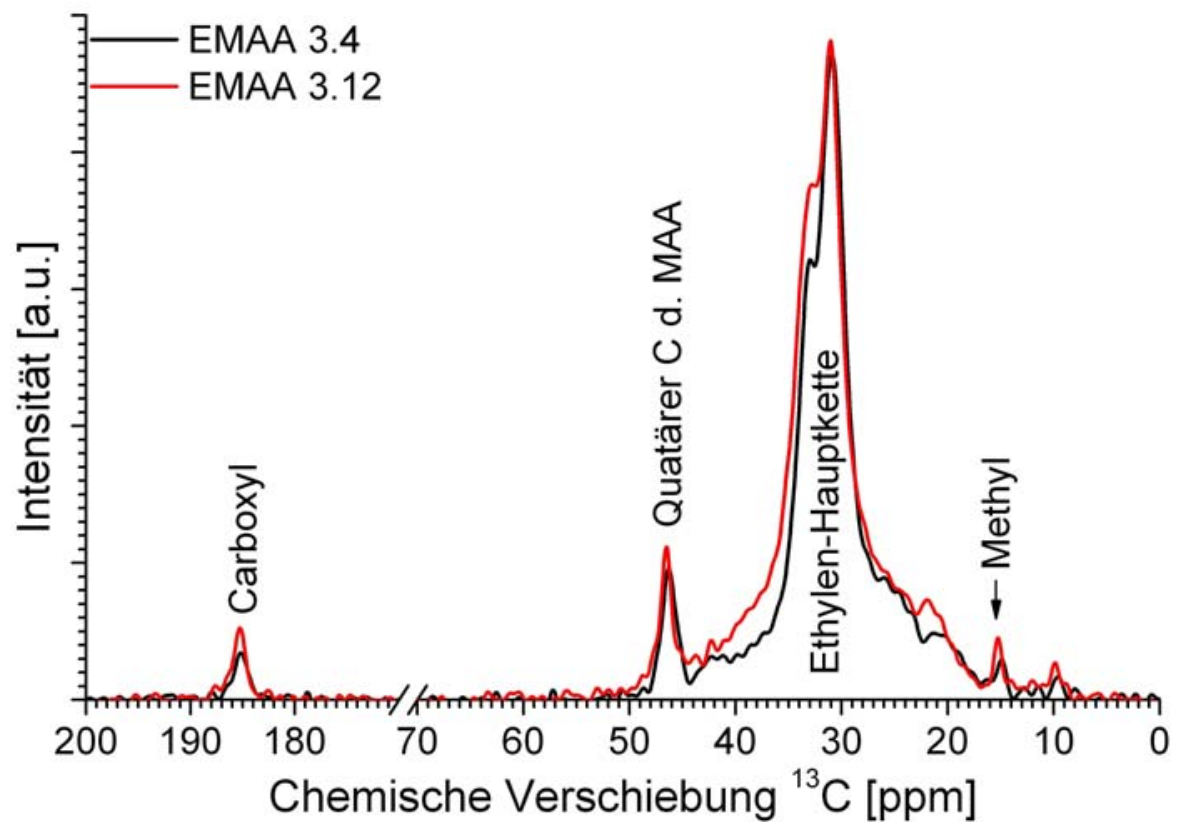

Abb. 5.1.3: NMR-Spektrum zweier EMAA-Proben der dritten Syntheseserie

\section{Zusammenfassung der synthesebedingten Probeneigenschaften}

Die Ergebnisse der Bestimmung des MAA-Gehalts aller im Folgenden analysierten Proben zeigt Abbildung 5.1.4. Es ist zu erkennen, dass eine höhere MAA-Fördermenge zu einem höheren MAA-Gehalt im Copolymer führt. Die geschätzte Standardabweichung aller Analysemethoden zusammen beträgt etwa 2 mol\%. Die Konzentrationen der FTIR-Messungen an den Folien sind systematisch höher als die der ATR-FTIR, welche ihrerseits höher sind als die durch NMR bestimmten Werte. Der MAA-Gehalt der Serie 12 ließ sich nur mittels NMR bestimmen. Eine signifikante Abhängigkeit des MAA-Gehalts vom Synthesedruck wird unter Zuhilfenahme aller Messungen an Proben, die bei weiteren Drücken hergestellt worden sind, nicht beobachtet [BS07]. 
Tab. 5.1.2: Tabelle der Polymerisationsparameter (identisch zu Tab. 3.2.1)

\begin{tabular}{|l|l|l|l|l|l|l|}
\hline $\begin{array}{l}\text { Proben- } \\
\text { bezeich- } \\
\text { nung }\end{array}$ & $\begin{array}{l}\text { MAA- } \\
\text { Fluss } \\
{[\mathrm{g} / \mathrm{h}]}\end{array}$ & $\begin{array}{l}\text { Fluss } \\
\text { Initiator- } \\
\text { Lösung } \\
{[\mathrm{ml} / \mathrm{h}]}\end{array}$ & $\begin{array}{l}\text { Initiator- } \\
\text { konzen- } \\
\text { tration } \\
{[\mathrm{mmol} / \mathrm{l}]}\end{array}$ & $\begin{array}{l}\text { Synthese- } \\
\text { druck } \\
{[\mathrm{bar}]}\end{array}$ & $\begin{array}{l}\text { Mantel- } \\
\text { tempera- } \\
\text { tur }[\mathrm{K}]\end{array}$ & $\begin{array}{l}\text { Innen- } \\
\text { tempera- } \\
\text { tur }[\mathrm{K}]\end{array}$ \\
\hline $\begin{array}{l}\text { EMAA } \\
1.2\end{array}$ & 34,2 & 23,1 & $\begin{array}{l}\text { TxF: } 23,2 \\
\text { TxB: } 6,93\end{array}$ & 2000 & 515 & 528 \\
\hline $\begin{array}{l}\text { EMAA } \\
2.2\end{array}$ & 34,2 & 27,2 & $\begin{array}{l}\text { TxF: } 23,4 \\
\text { TxB: } 7,03\end{array}$ & 1860 & 518 & $544-549$ \\
\hline $\begin{array}{l}\text { EMAA } \\
2.10\end{array}$ & 34,2 & 57,9 & $\begin{array}{l}\text { TxF: } 23,4 \\
\text { TxB: } 7,03\end{array}$ & 1300 & 519 & $541-545$ \\
\hline $\begin{array}{l}\text { EMAA } \\
3.4\end{array}$ & 116,0 & 94,0 & $\begin{array}{l}\text { TxF: } 23,2 \\
\text { TxB: } 6,91\end{array}$ & 2300 & 517 & 545 \\
\hline $\begin{array}{l}\text { EMAA } \\
3.12\end{array}$ & 116,0 & 455,0 & $\begin{array}{l}\text { TxF: } 23,2 \\
\text { TxB: } 6,91\end{array}$ & 1300 & 518 & $541-544$ \\
\hline $\begin{array}{l}\text { EMAA } \\
12.4\end{array}$ & 5,3 & 9,9 & TxF: 46,5 & 1300 & 518 & $536-537$ \\
\hline $\begin{array}{l}\text { EMAA } \\
12.11\end{array}$ & 5,3 & 2,4 & TxF: 46,5: & 2300 & 519 & $541-548$ \\
\hline
\end{tabular}

*: Angegeben ist die Konzentration in der zugesetzten Initiator-Lösung

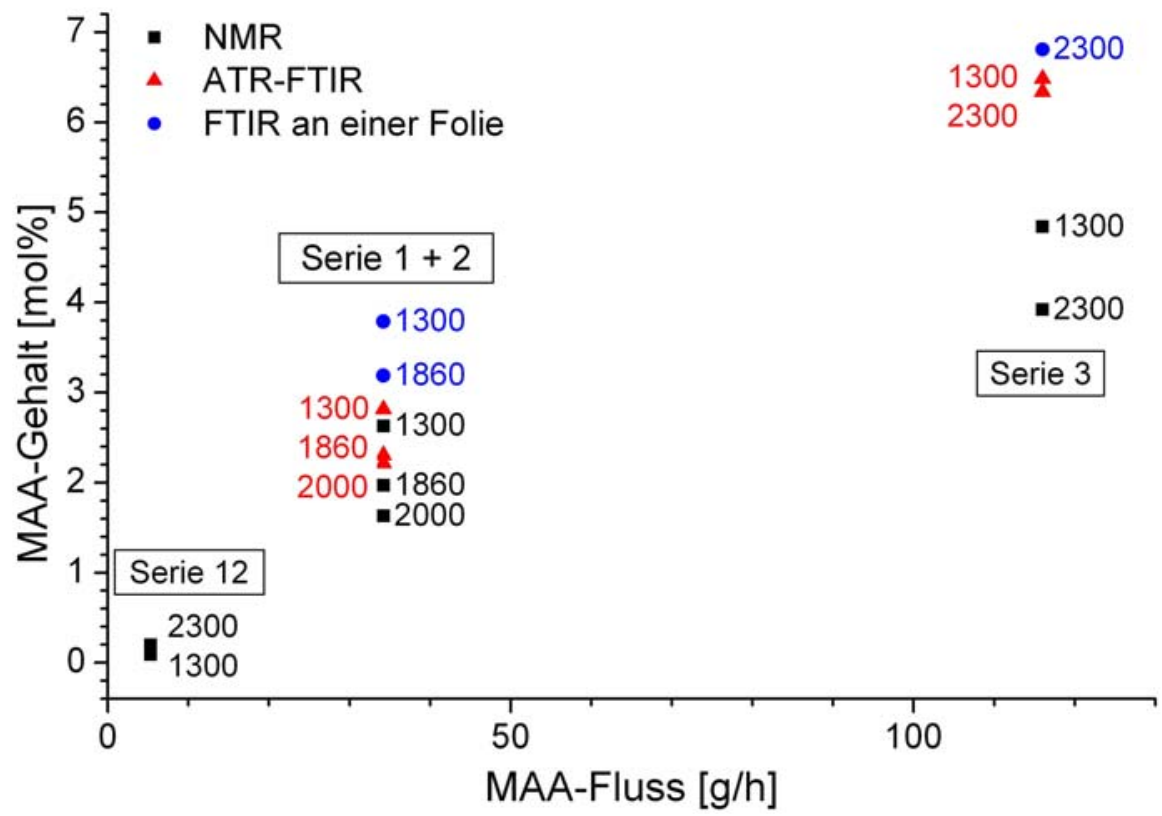

Abb. 5.1.4: MAA-Gehalt der untersuchten Proben in Abhängigkeit der Synthesebedingungen (Angabe des Synthesedrucks in bar)

Der Zusammenhang zwischen MAA-Fluss und -Gehalt ist nicht streng linear, da bei höherem MAA-Fluss auch mehr Initiator benötigt wird. Ein erhöhter Initiatorfluss hat einen höheren Umsatz an Monomeren insgesamt zur Folge, wie in Abbildung 5.1.5 gezeigt. Dies erhöht den 
Ethylen-Anteil, wodurch der MAA-Gehalt im Copolymer nicht in demselben Maße steigt, in dem der MAA-Fluss erhöht wird. Der Initiatorfluss ist dabei die Summe der beiden, nicht weiter gewichteten Initiator-Flüsse, unter Berücksichtigung der Konzentrationsverhältnisse in den Initiatorlösungen, wie in Tabelle 5.1.2 aufgelistet.

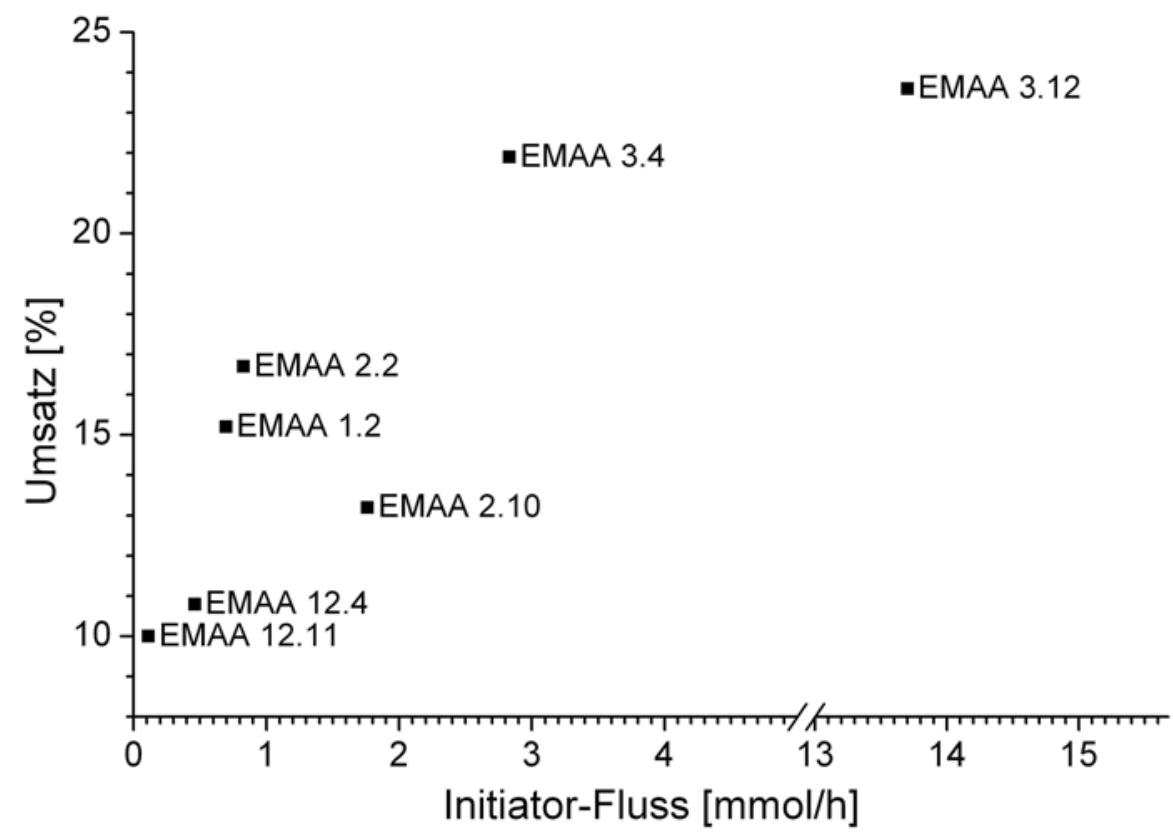

Abb. 5.1.5: Einfluss der Initiatorfördermenge auf den Umsatz der Synthese

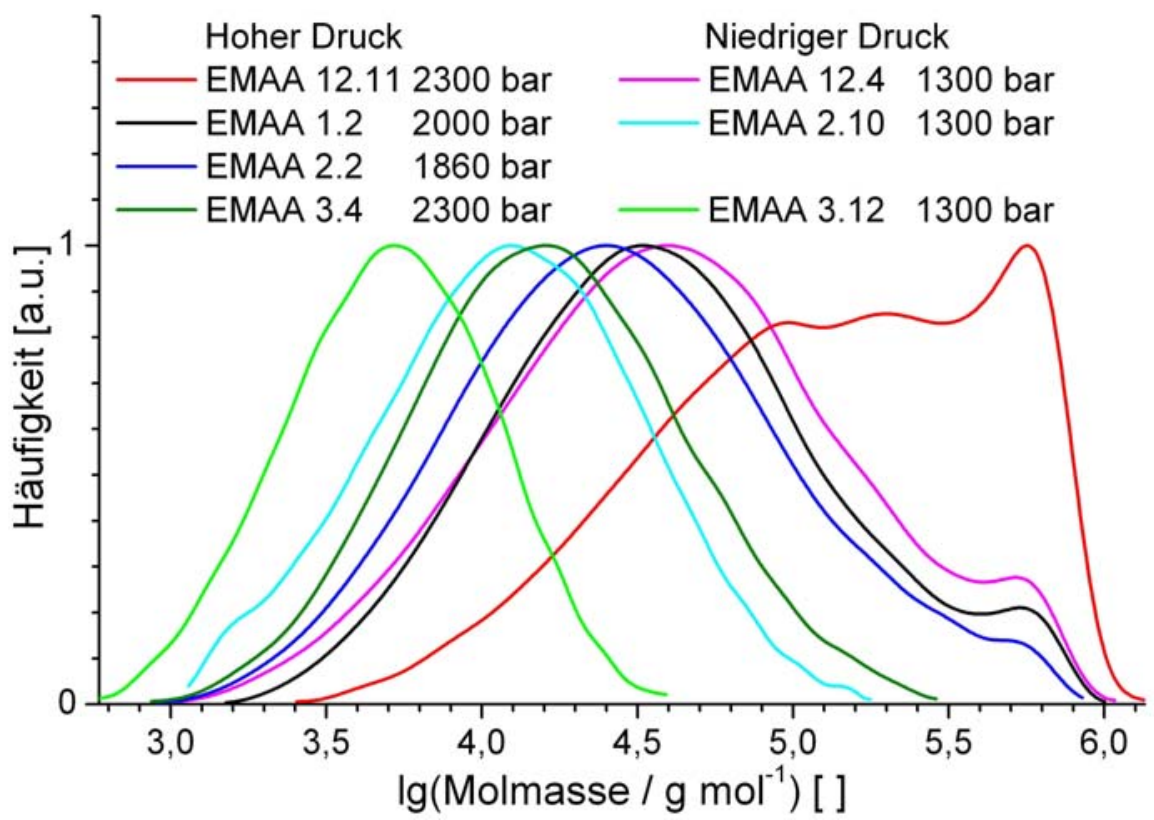

Abb. 5.1.6: Molmassenverteilung der hergestellten Proben in Abhängigkeit der Synthesebedingungen

Eine Erhöhung des Initiatorflusses führt zu einer Verkürzung der Kettenlänge, wie in Abbildung 5.1.6 gezeigt. Diese Systematik wird nur von den Proben EMAA 2.10 und 3.4 gebrochen, die bei stark verschiedenen Drücken hergestellt wurden. Bei Proben mit einem Maximum in der Häufigkeit für Ketten, deren logarithmierte Molmasse den Wert 4,3 übersteigt, 
wird bei etwa 5,7 ein zweites Maximum beobachtet. Bei der Probe EMAA 12.11 mit dem niedrigsten Initiatorgehalt und der größten Molmasse ist dieses Maximum sogar das höchste, und es zeigen sich zwei kleinere Maxima bei 5,0 und 5,3.

Die mittleren Molmassen der gekauften, industriell hergestellten Proben mit bekannter MAAKonzentration zeigen eine Molmassenverteilung, die mit den in Serie 1 und 2 hergestellten EMAA-Proben vergleichbar sind, obwohl sie deutlich mehr MAA enthalten. Die Breite der Verteilung ist schmaler als die der selbst hergestellten Proben, insbesondere bei der industriellen Probe mit 10,0 mol\% MAA.

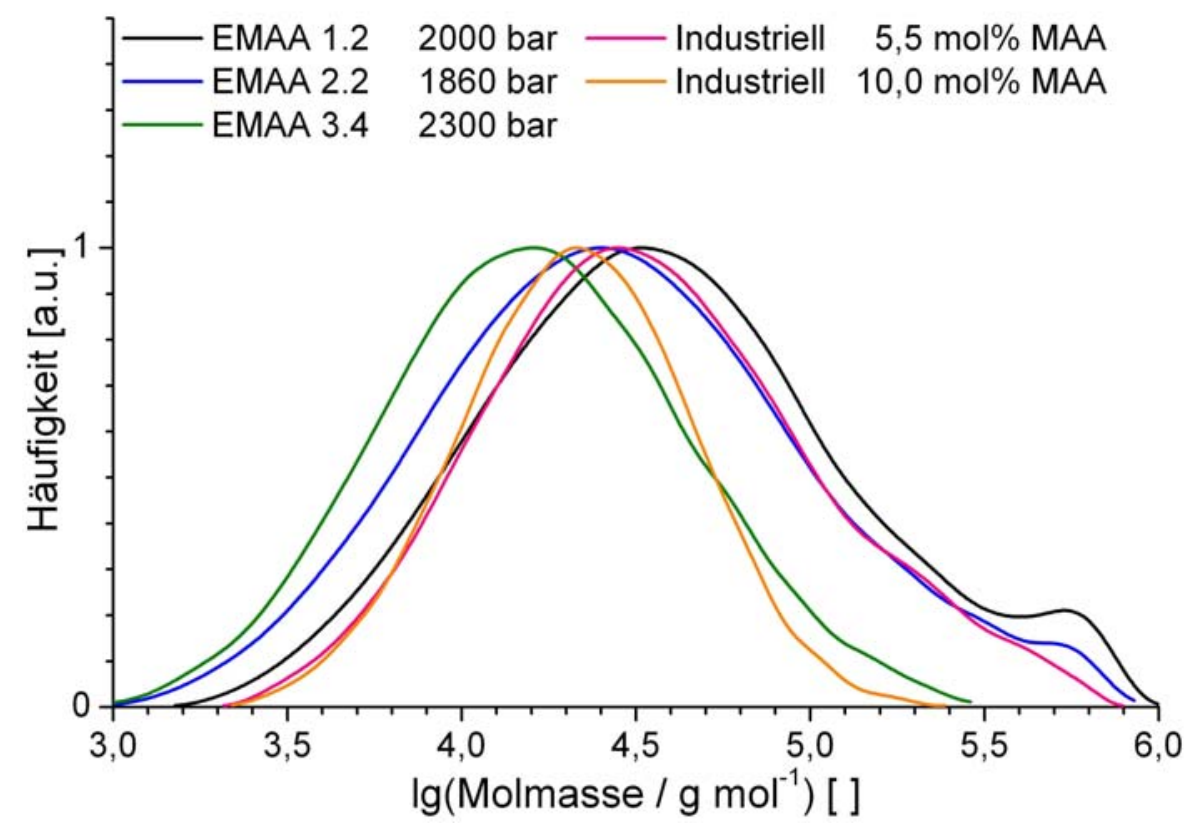

Abb. 5.1.7: Vergleich der Molmassenverteilungen mit den industriellen Proben

\subsection{DSC}

Die differentielle Kalorimetrie an den Polymerproben dient als ergänzende Methode zu den dynamischen mechanischen Messungen und zeichnet sich durch die Möglichkeit aus, mit vergleichbar wenig Aufwand einen ersten Überblick über die vorherrschenden Materialumwandlungen zu geben. Da zur Kühlung des Kalorimeters nur eine Ethylen-Kältemischung zur Verfügung steht, ist der zugängliche Temperaturbereich, wie in Kapitel 3.6 beschrieben, auf Temperaturen oberhalb $218 \mathrm{~K}$ beschränkt. Bei einer Heizrate von $5 \mathrm{~K} / \mathrm{min}$ wird ein stabiler Zustand mit konstanter Rate bei Temperaturen oberhalb ca. $230 \mathrm{~K}$ erreicht. Wie bei den Messungen an metallischen Proben (vgl. Kap. 4.3), dient als Referenz auch hier ein leerer Tiegel. Es ist aber nicht sinnvoll, eine zweite, identisch durchgeführte Aufheizkurve als Basislinie abzuziehen, da es nicht zu einer definierten, irreversiblen und vollständigen Kristallisation kommt. Um dennoch eine Vergleichbarkeit der Messungen des Wärmeflusses zu erreichen, werden alle Proben bis auf Temperaturen oberhalb des Übergangs in den Liquiduszustand erhitzt, und eine Gerade an dessen Verlauf angepasst. Diese wird anschließend als Basislinie von den Rohdaten abgezogen und die Differenz auf die zuvor bestimmte Probenmasse normiert. 
Diese Methode wird bei allen Messungen außer beim Homopolymer Polymethacrylsäure (PMAA) angewendet, da bei dem verwendeten PMAA die Zersetzungstemperatur unterhalb der Schmelztemperatur liegt. Die Zersetzung zeigt sich in einem Farbübergang von transparent zu gelb, zu braun und schließlich zu schwarz. Als Basislinie dient ein konstanter Wert, der dem Minimum der PMAA-Messung bei $234 \mathrm{~K}$ entspricht. Um diese Messung besser mit der des Polyethylen (PE) vergleichen zu können, ist der normierte Wärmefluss der PEMessung mit dem Faktor 0,25 skaliert, wie in Abbildung 5.2.1 gezeigt. PMAA zeigt einen sehr breiten Anstieg des normierten Wärmeflusses mit einem durch Tangentenkonstruktion (vgl. Abb. 4.3.3) ermittelten Onset bei ca. $319 \mathrm{~K}$, der in ein Maximum bei $385 \mathrm{~K}$ mündet. Nach Durchlaufen eines flachen Minimums steigt der Wärmefluss des PMAA im nicht mehr aufgetragenen Bereich oberhalb $420 \mathrm{~K}$ mit stetig wachsender Steigung an. PE dagegen zeigt ein klares Schmelzmaximum bei 394 K, dessen Onset bei ca. 388 K liegt. Nur in der Vergrößerung erkennbar ist eine weitere Stufe mit Onset bei ca. $311 \mathrm{~K}$.

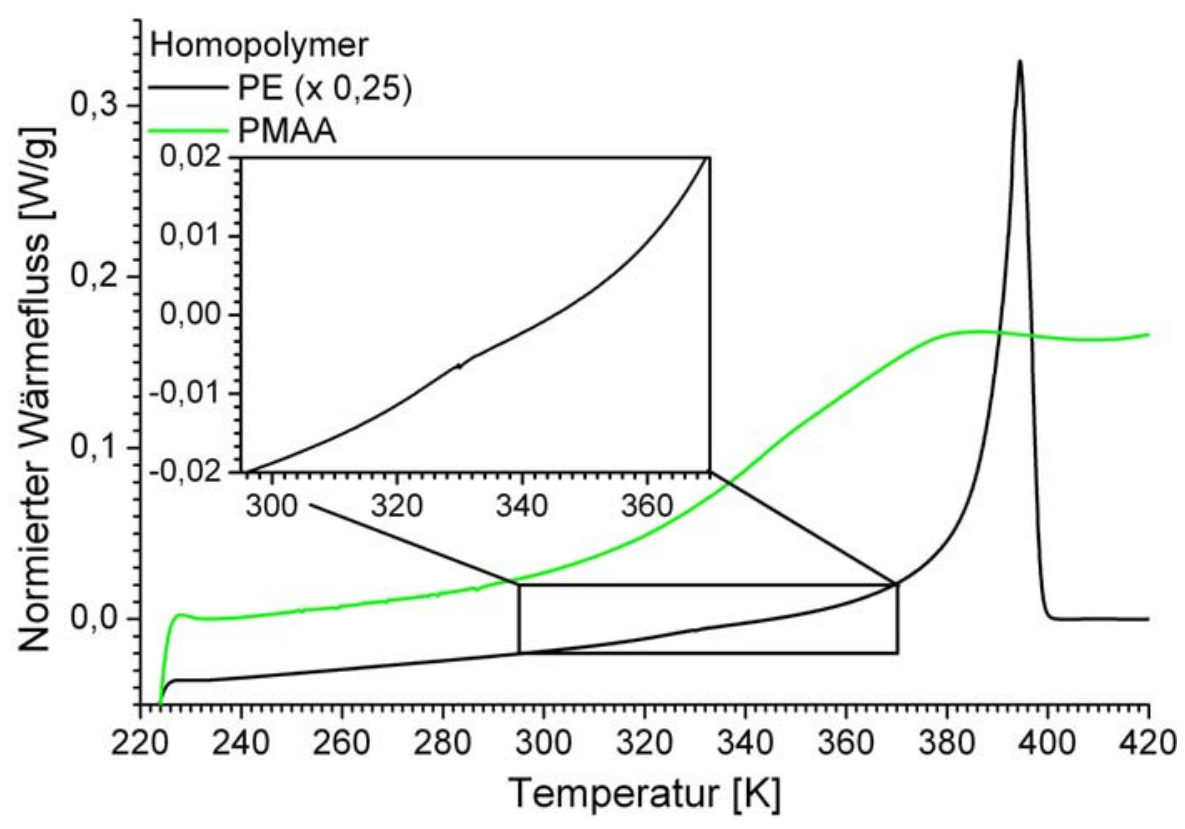

Abb. 5.2.1: DSC-Messung bei $5 \mathrm{~K} / \mathrm{min}$ an den Homopolymeren PE und PMAA. 
Die Verläufe der Wärmeflüsse der differentiellen Kalorimetrie an den synthetisierten EMAAProben zeigen die Abbildungen 5.2.2-4 in der Reihenfolge steigenden MAA-Gehalts. Die Darstellung erfolgt dabei in Rot für Proben mit niedrigem und in Blau oder Schwarz für hohen Synthesedruck.

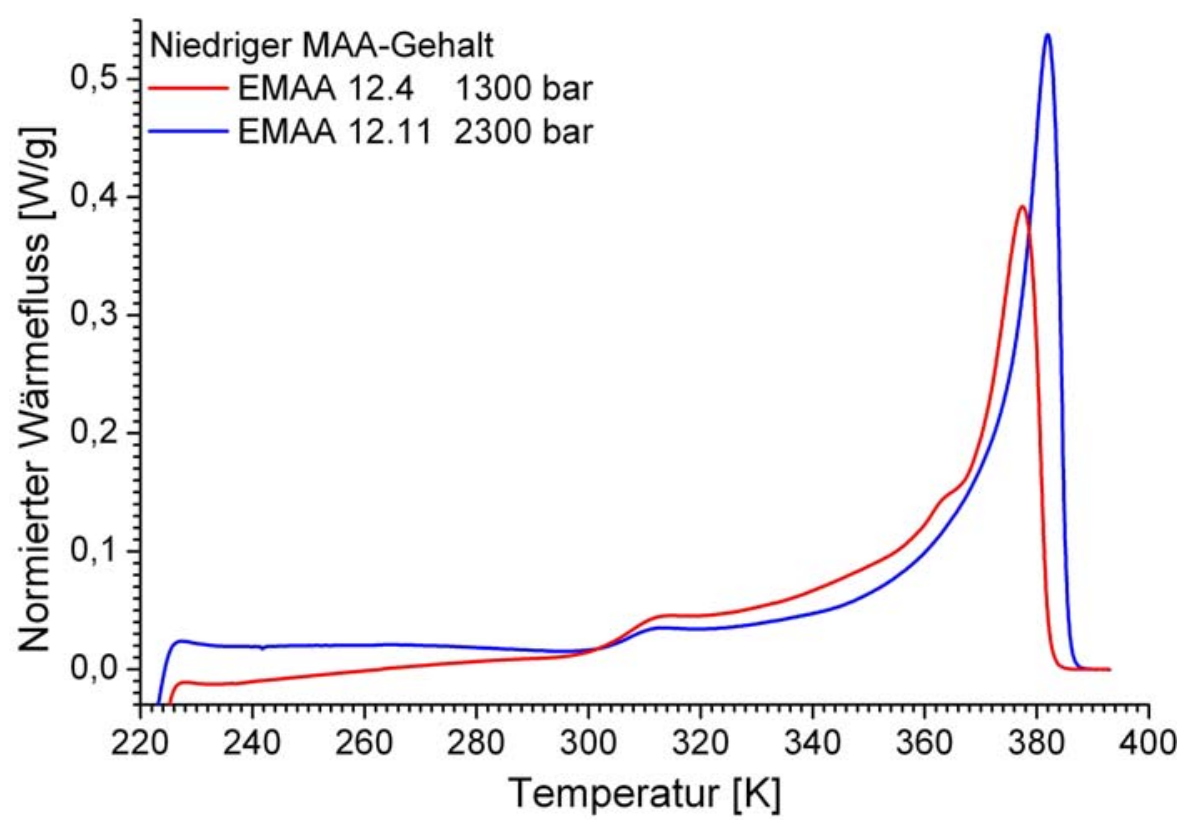

Abb. 5.2.2: DSC-Messung bei $5 \mathrm{~K} / \mathrm{min}$ an EMAA mit niedrigem MAA-Gehalt und stark verschiedenen Synthesedrücken

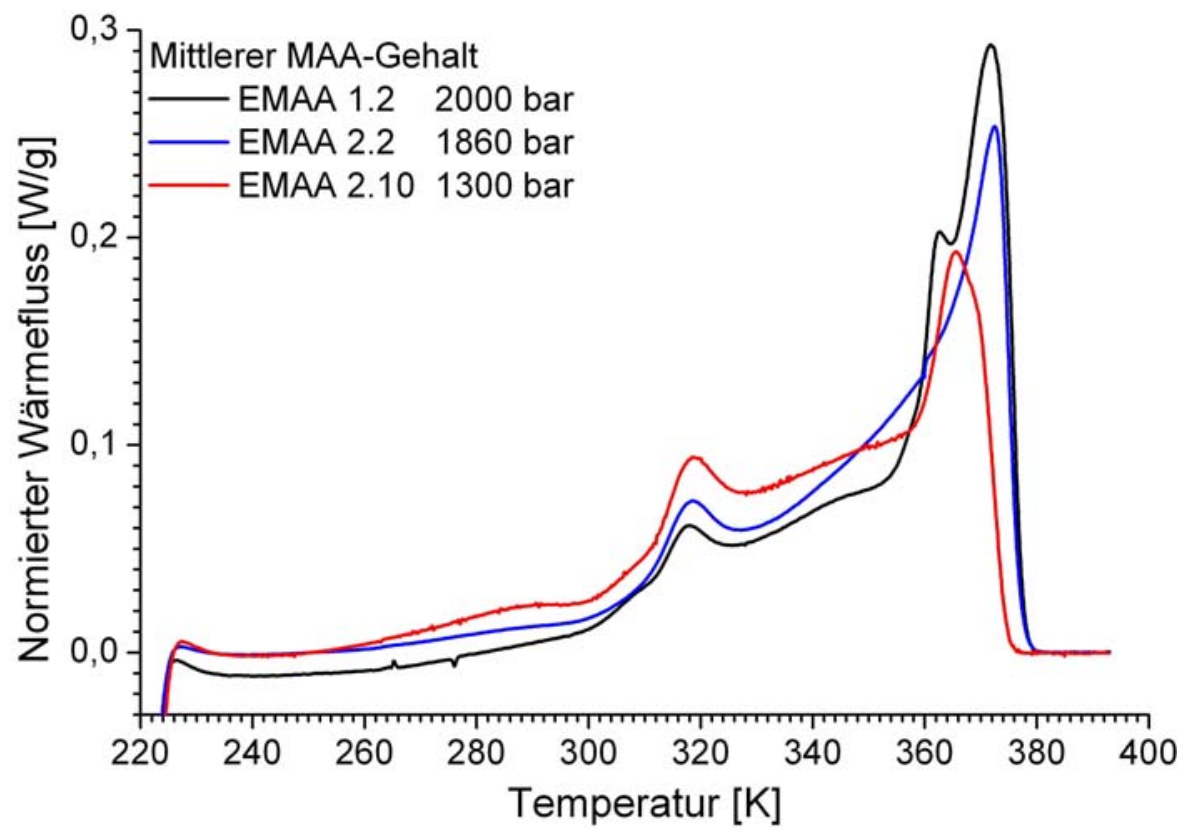

Abb. 5.2.3: DSC-Messung bei $5 \mathrm{~K} / \mathrm{min}$ an EMAA mit mittlerem MAA-Gehalt und stark verschiedenen Synthesedrücken 


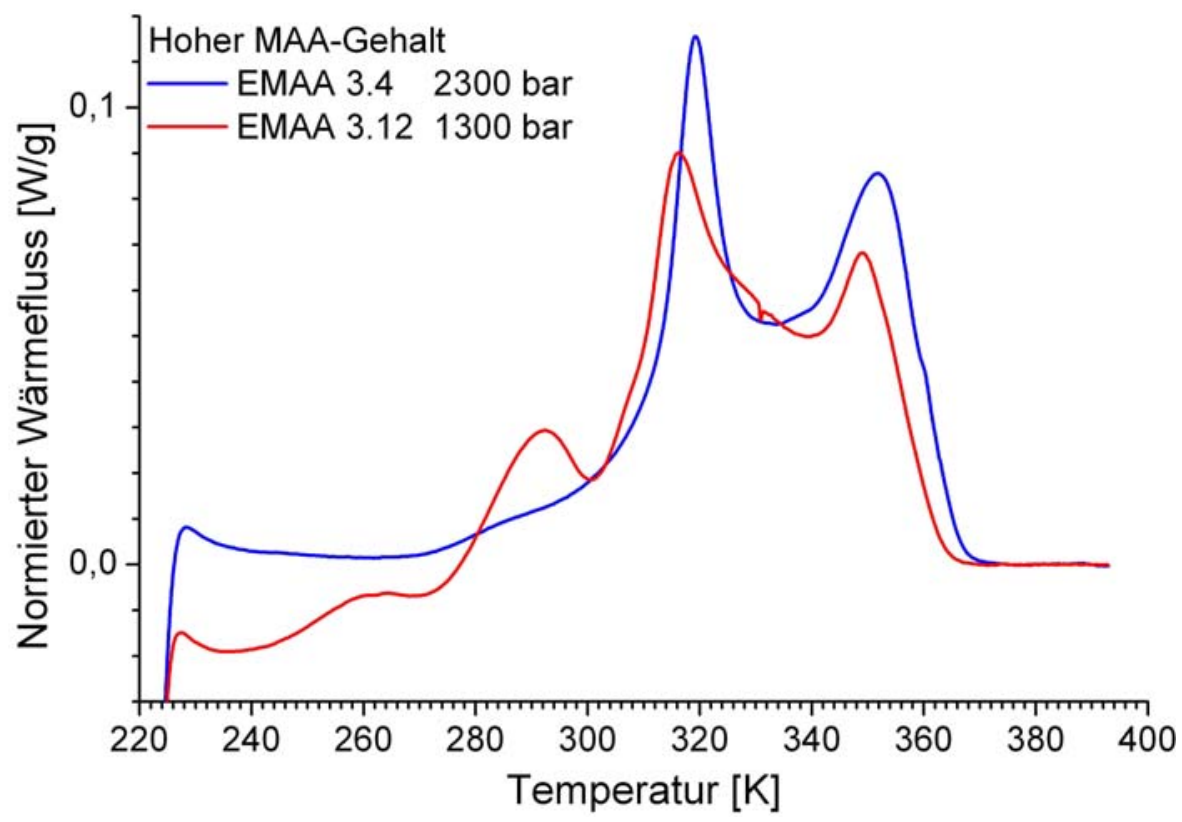

Abb. 5.2.4: DSC-Messung bei $5 \mathrm{~K} / \mathrm{min}$ an EMAA mit hohem MAA-Gehalt und stark verschiedenen Synthesedrücken

Beim Vergleich der differentialkalorimetrischen Messungen an EMAA fällt zunächst auf, dass mit steigendem MAA-Gehalt insbesondere der Verlauf des normierten Wärmeflusses von bei niedrigerem Druck synthetisierten Proben an Struktur gewinnt. Zur späteren Diskussion der Vorgänge ist es aufgrund deren Vielzahl sinnvoll, eine Nomenklatur einzuführen. Diese wird hier beispielhaft am Verlauf der Probe EMAA 3.12 in Abbildung 5.2.4 von hohen Temperaturen kommend durchgeführt. Oberhalb von $370 \mathrm{~K}$ zeigt sich ein Plateau, das bei allen Proben außer PMAA beobachtet wird. Das Maximum bei $349 \mathrm{~K}$ wird als $T_{\alpha 2}$ und dessen Onset bei $343 \mathrm{~K}$ als $T_{G 2}$ bezeichnet. Ist es, wie bei EMAA 1.2 in Abbildung 5.2.3, in zwei Spitzen aufgespaltet, so bezieht sich die Bezeichnung auf das an Intensität höhere. Das Maximum bei $325 \mathrm{~K}$ erhält die Bezeichnung $T_{\alpha}$ und dessen Onset bei $310 \mathrm{~K} T_{G}$. Unterhalb $T_{G}$ zeigen sich nur bei dieser Probe weitere Maxima, die bei allen anderen Messungen, wenn überhaupt, nur als Stufe auszumachen sind. Die Nomenklatur folgt mit sinkender Temperatur dem griechischen Alphabet; ihre Maxima müssten somit mit $\beta$ und $\gamma$ bezeichnet werden. Sofern sich Onsets bestimmen lassen, erhalten sie die Bezeichnung $T_{H}$ und $T_{I}$, die bei EMAA 3.12 die Werte $275 \mathrm{~K}$ und $243 \mathrm{~K}$ haben. In Abbildung 5.2.2 bei niedrigem MAAGehalt ist in diesem Bereich nur zu erkennen, dass die bei niedrigem Druck synthetisierte Probe mit steigender Temperatur einen schwachen Anstieg des normierten Wärmeflusses besitzt, während die Hochdruckprobe einen konstanten Verlauf aufweist. Bei mittlerem MAAGehalt (Abb. 5.2.3) zeigt sich eine Stufe für die Probe mit niedrigstem Synthesedruck und bei hohem MAA-Gehalt (Abb. 5.2.4) ist die Stufe bei niedrigerem Synthesedruck deutlich stärker ausgeprägt als bei hohem. Ein $\gamma$-Maximum zeigt nur EMAA 3.12.

Die Untersuchung der industriell hergestellten EMAA-Proben in Abbildung 5.2.5 zeigt keine erkennbaren Stufen unterhalb von $T_{G}$. Die $\alpha$ - und $\alpha 2$-Maxima sind sehr unsymmetrisch. Oberhalb 376 K zeigt sich bei der industriellen Probe mit 10,0 mol\% MAA-Gehalt ein niedriges und scharfes, zusätzliches Maximum. 


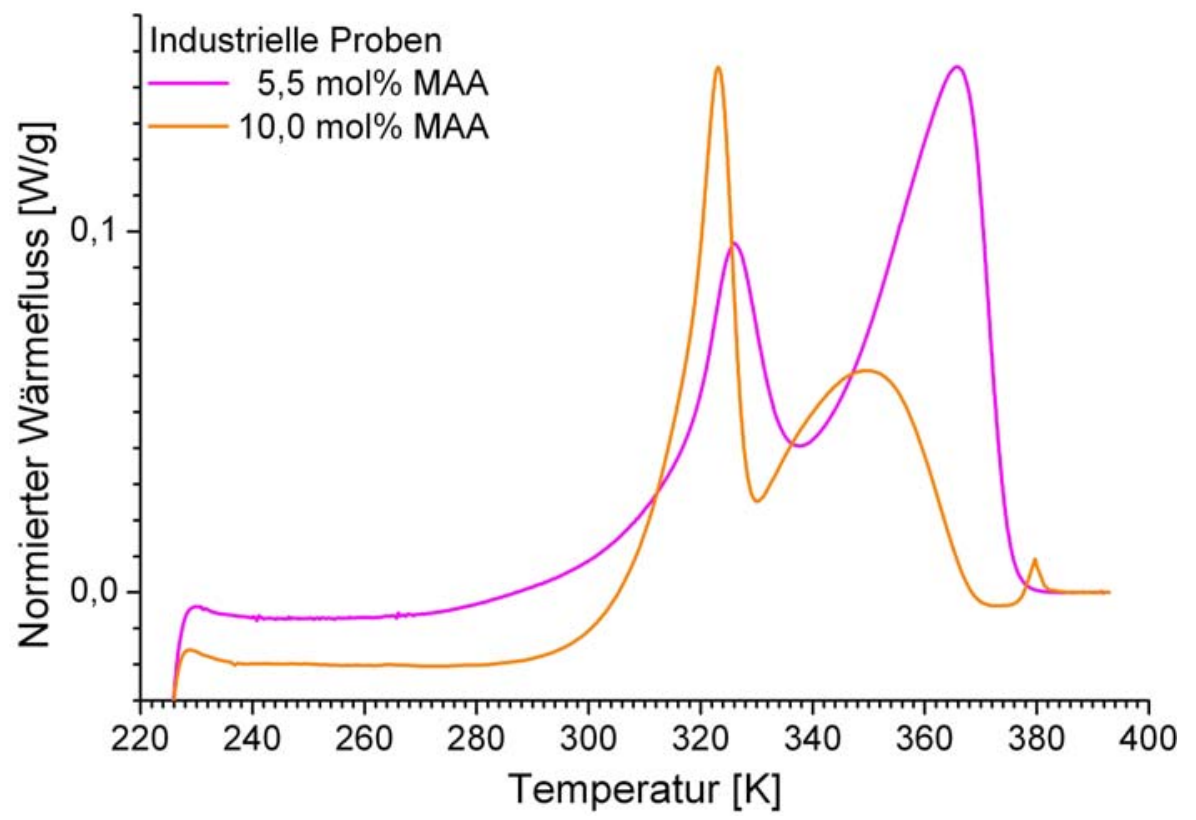

Abb. 5.2.5: DSC-Messung bei $5 \mathrm{~K} / \mathrm{min}$ an industriellem EMAA mit unbekannten Syntheseparametern

Abbildung 5.2.6 zeigt die charakteristischen Temperaturen $T_{G}, T_{\alpha}$ und $T_{\alpha 2}$ als Funktion der mit maximaler Häufigkeit vorkommenden logarithmierten Molmasse. Die Farbgebung ist dabei konsistent zu den vorangegangenen Abbildungen 5.2.2-5 aufgeteilt in Proben mit niedrigem und hohem Synthesedruck. Die industriellen Proben sind separat eingezeichnet.

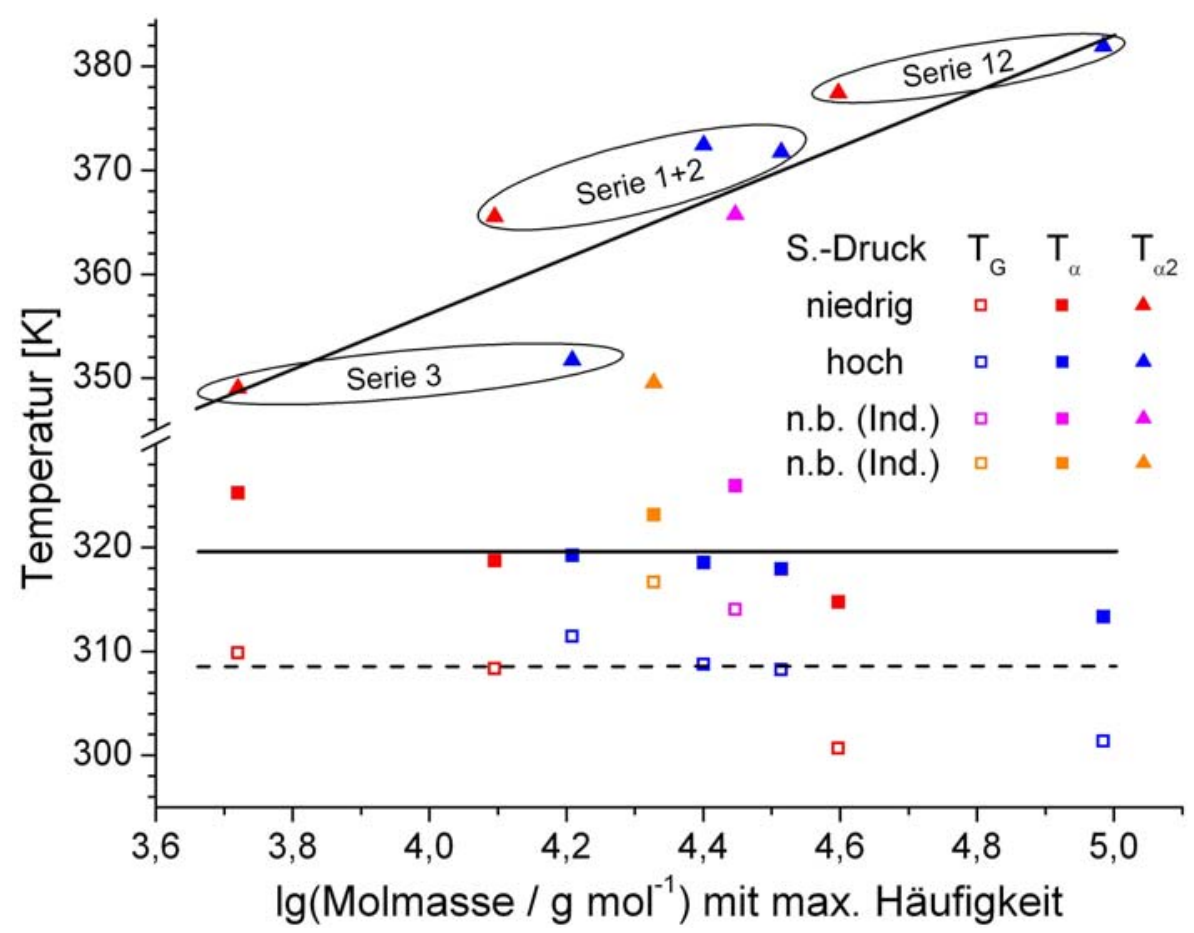

Abb. 5.2.6: Vergleich der charakteristischen Temperaturen in Abhängigkeit der Molmasse

Bei Probe 12.11 bezieht sich die Molmasse auf das erste, niedermolekularste Maximum in der Molmassenverteilung. Nicht eingezeichnet sind die Homopolymere, wobei alle drei charakteristischen Temperaturen für PE oberhalb der für EMAA gefundenen Werte liegen. Die einge- 
zeichneten Trendlinien sind eine Hilfestellung für das Auge und keine Ausgleichsgeraden. Es ist deutlich $\mathrm{zu}$ erkennen, dass mit steigender Molmasse eine Erhöhung von $T_{\alpha 2}$ einhergeht, während die Änderung von $T_{G}$ und $T_{\alpha}$ nicht signifikant ist, die Steigung einer linearen Fitfunktion wäre tendenziell negativ.

Zur Überprüfung der Reproduzierbarkeit der Messungen, und um zu untersuchen, ob die Vorgänge im Material reversibel durchlaufen werden können, ist es hilfreich, die Messungen mit abgestuften Heizrampen zu wiederholen. Dabei wird ein und dieselbe Probe mit $5 \mathrm{~K} / \mathrm{min}$ aufgeheizt, mit gleicher Soll-Rate abgekühlt und zur nächsten, höheren Temperatur aufgeheizt. Dazu eignen sich am besten die Proben der Serie 3, da diese die deutlichsten Effekte zeigen. Die bereits in Abbildung 5.2.4 gezeigten Messkurven sind in den beiden folgenden Abbildungen 5.2.7-8 in Schwarz als Referenz eingetragen.

Das ersten Aufheizen der EMAA 3.4-Probe zeigt eine gute qualitative Übereinstimmung der Messkurve mit der Referenz in Bezug auf die Lage und die Form des $\alpha$ - und $\beta$-Maximums. Zur Normierung des Wärmeflusses wird für beide Heizvorgänge ein linearer Fit an die zweite Heizkurve zwischen $370 \mathrm{~K}$ und $390 \mathrm{~K}$ und das Probengewicht verwendet (analog zu den bisherigen Messungen, wie zu Beginn des Unterkapitels beschrieben). Abweichend sind eine steilere Steigung und ein niedriges, aber scharfes zusätzliches Maximum bei $266 \mathrm{~K} \mathrm{zu}$ beobachten. Das erste Aufheizen erfolgt bis $333 \mathrm{~K}$. Der darauf folgende zweite Durchlauf zeigt einen weniger deutlichen Anstieg der Steigung im Bereich des $\beta$-Prozess und eine deutliche Verbreiterung des $\alpha$-Maximums. Des Weiteren erscheint ein weiteres Maximum zwischen $\alpha$ und $\alpha 2$, dessen Form und Position im Wesentlichen reproduziert wird.

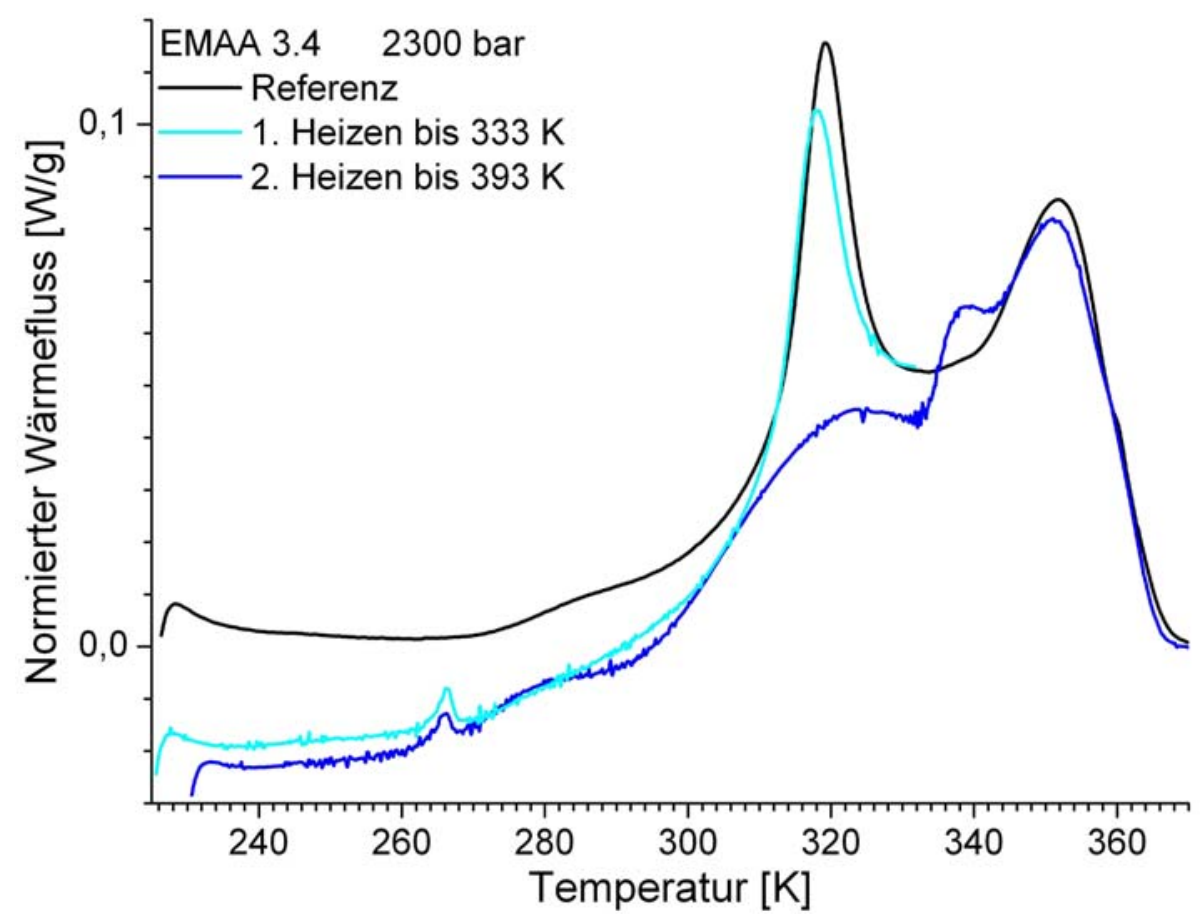

Abb. 5.2.7: Stufenweises Aufheizen bei $5 \mathrm{~K} / \mathrm{min}$ an EMAA mit hohem MAA-Gehalt und hohem Synthesedruck

Eine vergleichbare Beobachtung ist beim stufenweisen Heizen der Probe EMAA 3.12 zu machen. Der erste, zweite und dritte Lauf bis 273 K, 303 K und 333 K reproduzieren den Verlauf 
der Referenzkurve bis auf die Steigung. Es gibt kleine Abweichungen im zweiten Durchlauf bei ca. $280 \mathrm{~K}$ und den bereits bei EMAA 3.4 beobachteten Zusatzeffekt bei $266 \mathrm{~K}$. Beim vierten, an den bis $333 \mathrm{~K}$ anschließenden Aufheizvorgang, sind wiederum deutliche Änderungen zu beobachten. Der $\gamma$-Prozess und die Tieftemperaturflanke des $\beta$-Prozess werden reproduziert, letzterer wächst aber völlig mit dem $\alpha$-Prozess zu einem breiten Maximum zusammen. Auch hier kann ein weiteres Maximum zwischen $\alpha$ - und $\alpha 2$-Maximum beobachtet werden, wobei $\alpha 2$ wiederum gut in Form und Position mit der Referenz übereinstimmt.

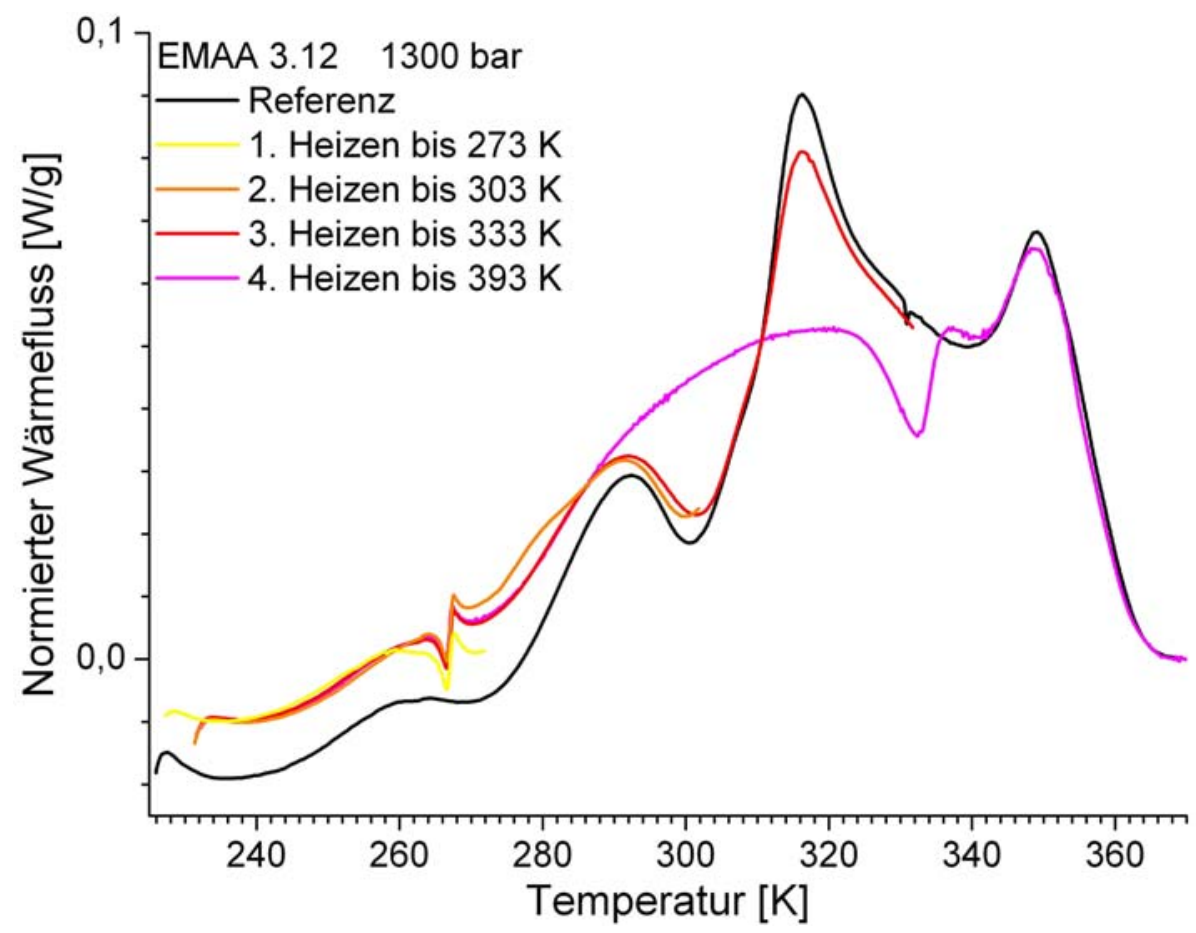

Abb. 5.2.8: Stufenweises Aufheizen bei $5 \mathrm{~K} / \mathrm{min}$ an EMAA mit hohem MAA-Gehalt und niedrigem Synthesedruck

\subsection{DMA}

\section{Effekt des Pressens}

Im Unterschied zu den in Kapitel 4.4f beschriebenen Messungen an metallischen Gläsern, erfolgt die mechanische Spektroskopie an Polymerproben in Kompression, wie in Kapitel 3.10 dargestellt. Vor Beginn der eigentlichen Messungen müssen die Proben dazu in eine zylindrische Form gebracht werden. Die genaue Prozedur des zweistündigen Pressens bei ca. $390 \mathrm{~K}$ und das anschließende Drehen der Probe aus der Form erläutert Kapitel 3.3.

Wie bereits bei der sensorischen in Augenscheinnahme der unbehandelten Copolymerproben, zeigen sich schon beim Einbau der fertigen Zylinder in die DMA deutliche Unterschiede. So sind die Proben der Serie 3 die deutlich duktilsten und es fällt auf, dass bei niedrigem Druck synthetisierte Proben ein höheres Dämpfungssignal zu Beginn der Messungen zeigen. Wie bei den im vorangegangen Abschnitt beschriebenen DSC-Untersuchungen ist es daher wichtig zu fragen, ob die Messungen reproduzierbar sind, und in wieweit sie von der Vorgeschichte der Probe bestimmt werden. Die größten Unterschiede während der spektroskopischen Untersu- 
chungen zeigen dabei die Proben der Serie 3, wobei auch hier mehrere Aufheizprozesse an einer Probe mit unterschiedlicher Probenhandhabung durchgeführt worden sind.

Ein zweifaches Aufheizen einer EMAA 3.4-Probe zeigt Abbildung 5.3.1. Die bei 2300 bar und hohem MAA-Fluss synthetisierte Probe wird dabei bei $350 \mathrm{mN}$ statischer Kraft und $300 \mathrm{mN}$ dynamischer Kraft bei $5 \mathrm{~Hz}$ und $5 \mathrm{~K} / \mathrm{min}$ bis $361 \mathrm{~K}$ geheizt, möglichst rasch wieder auf die Anfangstemperatur gekühlt (geschätzte Rate zu Anfang des Kühlvorgangs: $20 \mathrm{~K} / \mathrm{min}$ ) und erneut auf $364 \mathrm{~K}$ geheizt. Wichtig ist dabei, dass beim Kühlen keine Kräfte an der Probe anliegen und die Probe zwischen erstem und zweitem Lauf nicht ausgebaut wird. Beim ersten Durchlauf fällt der Speichermodul von anfänglich ca. $170 \mathrm{MPa} a b$, dessen Änderung sich ab ca. $184 \mathrm{~K}$ verlangsamt, bevor er mit einem Onset bei ca. $291 \mathrm{~K}$ über zwei Stufen stark abfällt. Im Verlustmodul zeigen sich dabei zwei Maxima um ca. $168 \mathrm{~K}$ und $299 \mathrm{~K}$. Im Bereich zwischen $185 \mathrm{~K}$ und $260 \mathrm{~K}$ liegt der Verlust unterhalb der DMA-Empfindlichkeit.

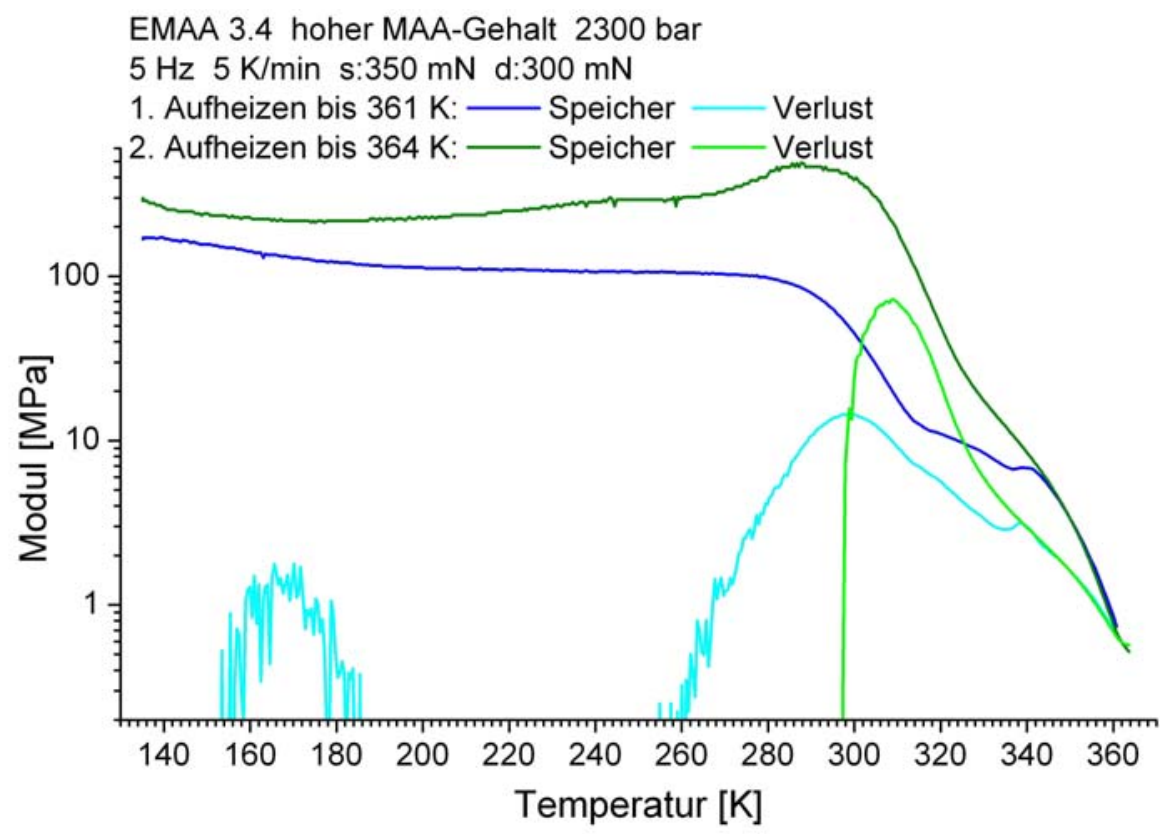

Abb. 5.3.1: Unterschiede bei der mehrfachen mechanischen Spektroskopie der bei 2300 bar synthetisierten EMAA-Probe mit hohem MAA-Gehalt

Beim zweiten Aufheizen wird ein deutlich höherer Anfangsspeichermodul von ca. $290 \mathrm{MPa}$ zu beobachten. Abweichend zum ersten Durchlauf ist weiterhin, dass nach einem anfänglichen Abfall der Speichermodul mit einem Onset bei ca. $160 \mathrm{~K} \mathrm{zu}$ steigen beginnt und sich bei $268 \mathrm{~K}$ noch einmal beschleunigt. An ein Maximum von $475 \mathrm{~K}$ bei $288 \mathrm{~K}$ schließt wiederum ein zweigeteilter Abfall des Speichermoduls an, wobei die Teilschritte aber weniger gut trennbar sind. Der zugehörige Verlustmodul ist unterhalb $297 \mathrm{~K}$ nicht messbar und beschreibt um 309 K ein Maximum.

Stark abweichend hierzu verhält sich die bei 1300 bar und hohem MAA-Fluss synthetisierte Probe EMAA 3.12 in Abbildung 5.3.2. Hier ist der umgekehrte Fall zu sehen. Der Speichermodul ist beim zweiten Durchlauf niedriger als beim ersten. Dies steht im Zusammenhang mit einer leicht verschiedenen Probenbehandlung zwischen den Heizzyklen. Nach dem ersten Aufheizen bis $344 \mathrm{~K}$ wird die Probe ebenfalls ohne Kraft abgekühlt, dann aber bei Zimmertemperatur von Stempel und Bodenplatte getrennt, an denen sie zu diesem Zeitpunkt festklebt. 
Nach kurzer Kontrolle der Probengeometrie wird die Probe wieder eingebaut, und es wird mit dem zweiten Aufheizen bis $347 \mathrm{~K}$ fortgefahren. Beim ersten Aufheizen zeigt diese EMAA 3.12-Probe einen anfangs ansteigenden, hohen Speichermodul von bis zu $330 \mathrm{MPa}$, der mit einem Onset bei $270 \mathrm{~K}$ in einem gleichfalls zweistufigen Vorgang abfällt. Der Verlustmodul ist bis $259 \mathrm{~K}$ zu klein und zeigt ein Maximum bei $277 \mathrm{~K}$.

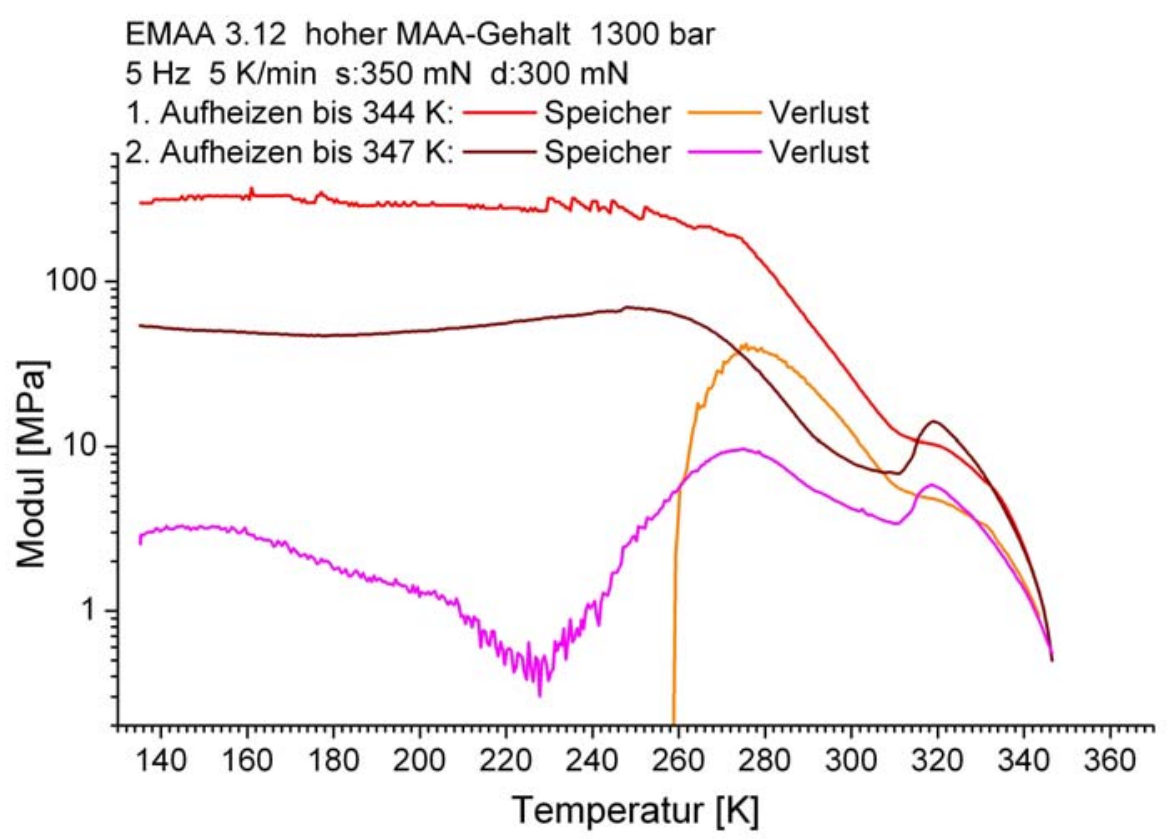

Abb. 5.3.2: Unterschiede bei der mehrfachen mechanischen Spektroskopie der bei 1300 bar synthetisierten EMAA-Probe mit hohem MAA-Gehalt

Beim zweiten Aufheizen ist das Material deutlich weicher und zeigt einen fallenden Speichermodul von anfänglich $54 \mathrm{MPa}$, weniger als ein Sechstel des ersten Durchlaufs. Nach dem Durchlaufen eines Minimums bei $178 \mathrm{~K}$ steigt dieser an bis zu einem Maximum von $69 \mathrm{MPa}$ bei 249 K. Der Abfall bei höheren Temperaturen geschieht auch hier in zwei Schritten, wobei in diesem Durchlauf ein deutliches Maximum bei $14 \mathrm{MPa}$ und $319 \mathrm{~K}$ zu erkennen ist. Ein derartiges Verhalten deutet sich auch im ersten Aufheizen der Probe 3.4 beim zweiten Abfall an. Mit dem niedrigeren Speichermodul geht ein Verlustmodul einher, der sich im gesamten Messbereich verfolgen lässt. Dieser zeigt zunächst ein breites Maximum bei ca. $149 \mathrm{~K}$, auf dessen Hochtemperaturflanke ein weiteres bei ca. $199 \mathrm{~K} \mathrm{zu}$ sehen ist. Auf einen Bereich mit niedrigem Verlustmodul folgt ein Anstieg zu einem relativ hohen, breiten Maximum bei 275 K. Ein weiteres, kleineres Maximum fällt mit der Temperatur des Maximums im Speichermodul bei $319 \mathrm{~K}$ zusammen.

\section{Existenz zusätzlicher Relaxationen}

Da im Folgenden bei der Untersuchung auf zusätzliche Relaxationsprozesse der Verlustmoduln von großem Interesse ist, werden in diesem Unterabschnitt Messungen an Homo- und Copolymeren gezeigt, die einen möglichst deutlichen und damit einhergehend niedrigen Verlustmodul aufweisen. Aufgrund der im vorangegangenen Abschnitt gezeigten großen Abhängigkeit der elastischen Moduln von der Vorgeschichte, ist es nicht sinnvoll, die Absolutwerte des Verlusts zu betrachten. Stattdessen wird im Folgenden der Verlauf des tan $\delta$ präsentiert, 
der auch alle Relaxationsprozesse zeigt, nicht aber von den Absolutwerten und Geometrieeffekten abhängt (vgl. Kap. 2.4). Die Abbildungen 5.3.3-7 zeigen einen Vergleich von allen untersuchten Homo- und Copolymeren bei $5 \mathrm{~Hz}$ Frequenz und $5 \mathrm{~K} / \mathrm{min}$ Heizrate und den darin angegebenen statischen (s) und dynamischen (d) Kräften.

Als besonders schwierig erweist sich dabei die Messung des Homopolymers PMAA, da dies in Form eines porösen, brüchigen Granulats vorliegt. Laut Herstellerangaben ist es zu einem hohen Prozentsatz kristallin und zerfällt auf chemischer Ebene vor Erreichen der Schmelztemperatur. Es lässt sich aber mit Wasser in einen formbaren Gelzustand bringen, wobei die Lagerung im Vakuum der mit einer Vorpumpe evakuierbaren Schleusenkammer eine Möglichkeit bietet, die Festigkeit zu kontrollieren. Auf diese, wenn auch relativ undefinierte Weise lässt sich ein in der DMA messbarer Probenzylinder präparieren.

Die wesentlichen Effekte sind ein Maximum im ansonsten in logarithmischer Auftragung linear ansteigenden Verlauf des tan $\delta$ des PE bei $155 \mathrm{~K}$, gezeigt in Abbildung 5.3.3. Des Weiteren ist ein scharfes Maximum bei $228 \mathrm{~K}$ zu beobachten. Dies beruht auf einem Platzen eines Silikonschlauches und enthält keine Aussagekraft in Hinblick auf Prozesse im Material. Im Folgenden sind die in der wasserarmen Argonatmosphäre extrem häufig platzenden Silikonschläuche durch Metallgewebeschläuche ersetzt worden(vgl. Kap. 3.10). PMAA zeigt eine breite Schulter um $235 \mathrm{~K}$ auf dem stetig ansteigenden Verlauf des $\tan \delta$.

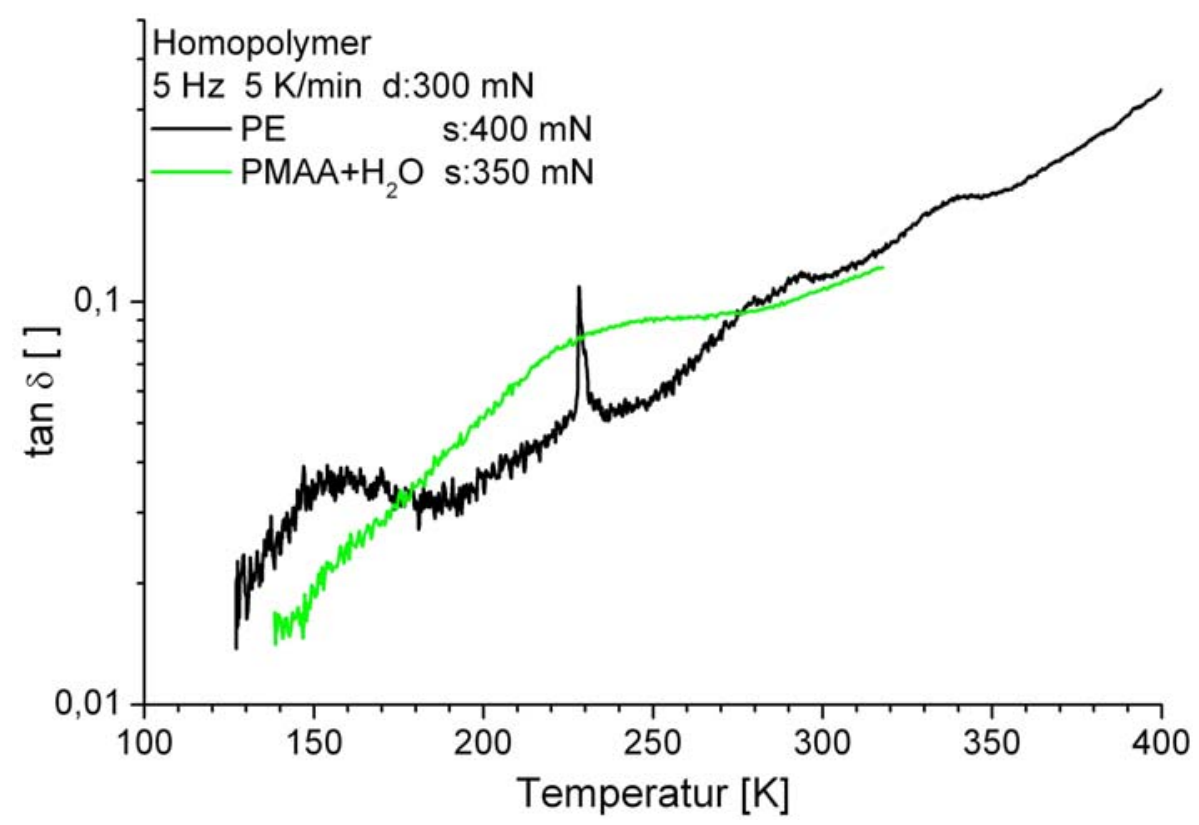

Abb. 5.3.3: Verlauf des tan $\delta$ bei der mechanischen Spektroskopie an PE und mit Wasser versetztem PMAA

Die in Abbildung 5.3.1+2 beobachtete Tendenz der besseren Messbarkeit der bei niedrigem Druck synthetisierten Proben zeigt sich auch in Abbildung 5.3.4 beim Vergleich der Proben EMAA 12.4 und 12.11, die bei niedrigem MAA-Fluss hergestellt sind. Bei $159 \mathrm{~K}$ wird für EMAA 12.4 ein Maximum beobachtet, wo 12.11 noch nicht messbar ist. Auch ist der Anstieg zum Maximum bei $277 \mathrm{~K}$ von 12.4 ist nicht so steil, wie der in eine Schulter bei ca. $285 \mathrm{~K}$ mündende Anstieg von 12.11. 
Dieser Trend setzt sich bei den Proben der Serie 1 und 2 mit mittlerem MAA-Gehalt in Abbildung 5.3.5 fort. Bei der Probe 1.2, die bei 2000 bar synthetisiert ist, zeigt sich nach einem nicht messbaren Bereich unterhalb $271 \mathrm{~K}$ ein Maximum bei $317 \mathrm{~K}$, das bei 2.2 bei $314 \mathrm{~K}$ und bei 2.10 bei $309 \mathrm{~K}$ liegt. Dabei haben die letzteren beiden ein Maximum im Bereich des ersten PE-Maximums bei 150 K (2.2) und 158 K (2.10) gemein. Darüber hinaus ist ein zusätzliches Maximum um $219 \mathrm{~K}$ im Verlauf der Probe 2.10 mit dem niedrigsten Synthesedruck zu sehen.

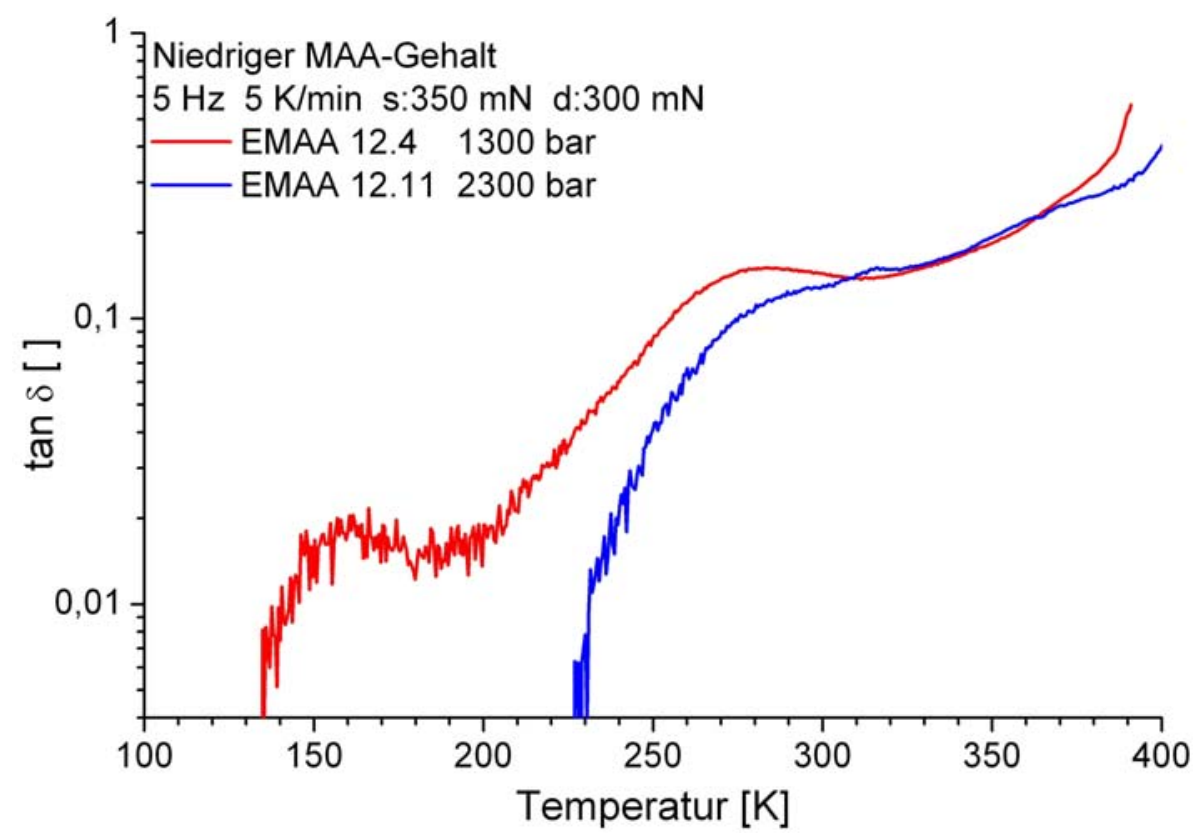

Abb. 5.3.4: Verlauf des $\tan \delta$ bei der mechanischen Spektroskopie der bei stark verschiedenen Drücken synthetisierten EMAA-Probe mit niedrigem MAA-Gehalt

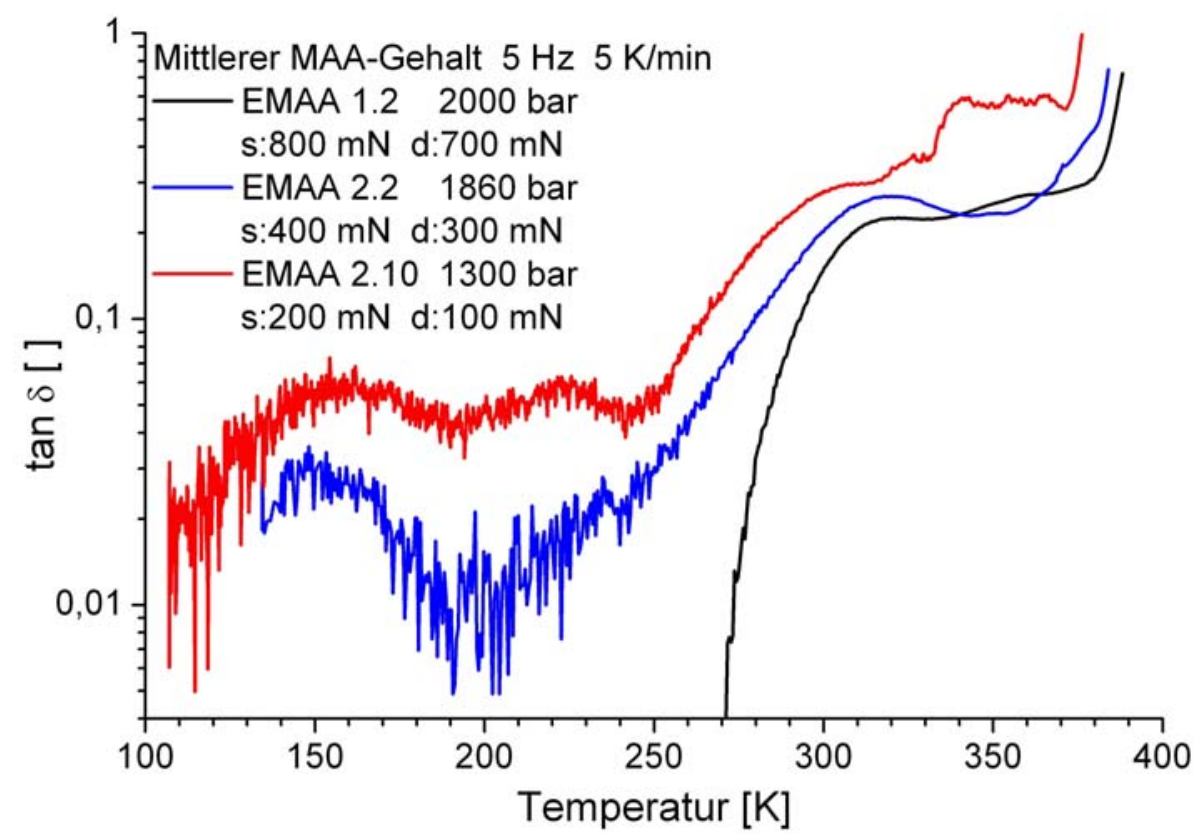

Abb. 5.3.5: Verlauf des $\tan \delta$ bei der mechanischen Spektroskopie der bei stark verschiedenen Drücken synthetisierten EMAA-Probe mit mittlerem MAA-Gehalt 


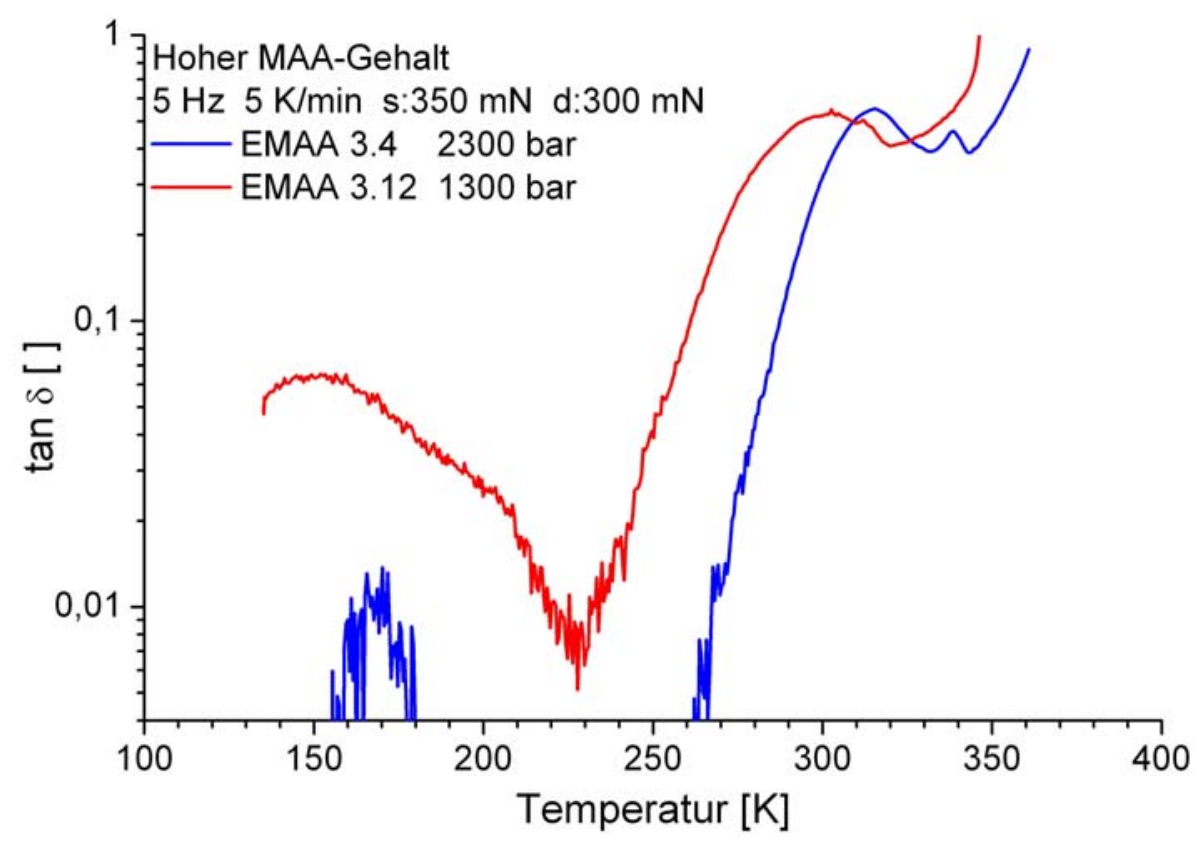

Abb. 5.3.6: Verlauf des $\tan \delta$ bei der mechanischen Spektroskopie der bei stark verschiedenen Drücken synthetisierten EMAA-Probe mit hohem MAA-Gehalt

Auch für die Proben der Serie 3 mit hohem MAA-Gehalt werden in Abbildung 5.3.6 Maxima im Bereich des PE-Maximums beobachtet. Dieser Effekt ist bei ca. $168 \mathrm{~K}$ sehr undeutlich für EMAA 3.4 zusehen, das bei 2300 bar synthetisiert worden ist und klarer bei $152 \mathrm{~K}$ für EMAA 3.12, welches bei 1300 bar synthetisiert worden ist. Letzteres weist eine Schulter um $199 \mathrm{~K}$ auf, wo 3.4 bereits nicht mehr messbar ist. 3.4 zeigt nach einem nicht messbaren Bereich bis $261 \mathrm{~K}$ einen steilen Anstieg zu einem Maximum bei $315 \mathrm{~K}$, gefolgt von einem kleineren bei $338 \mathrm{~K}$, wogegen 3.12 bereits bei $302 \mathrm{~K}$ ein Maximum beschreibt.

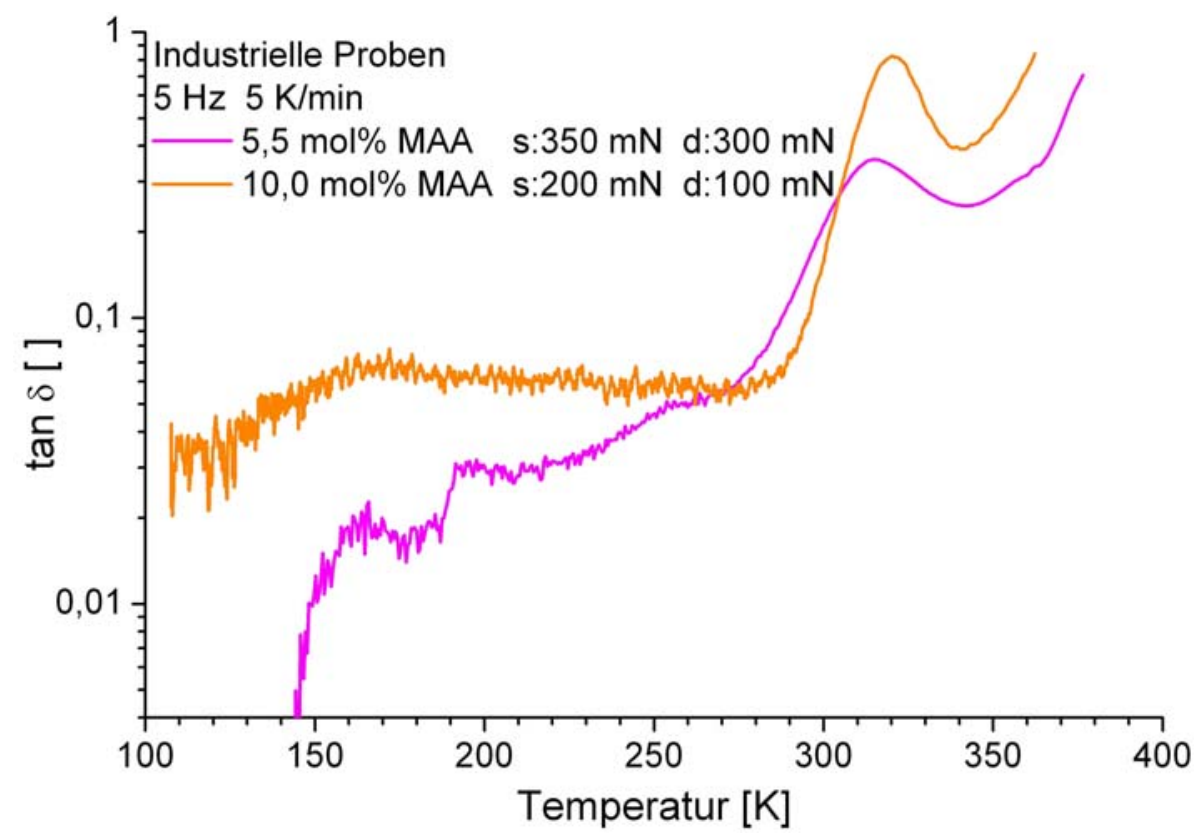

Abb. 5.3.7: Verlauf des tan $\delta$ bei der mechanischen Spektroskopie der industriellen, bei unbekannten Drücken synthetisierten EMAA-Probe mit hohem MAA-Gehalt 
Die industriell, bei unbekannten Prozessparametern hergestellten Proben zeigen ein nur bedingt vergleichbares Bild, wie in Abbildung 5.3.7 gezeigt. So besitzt die industrielle Probe mit 5,5 mol\% MAA-Gehalt oberhalb $145 \mathrm{~K}$ einen Anstieg zu einem undeutlichem Maximum bei etwa $164 \mathrm{~K}$, dem zwei weitere bei $198 \mathrm{~K}$ und $255 \mathrm{~K}$ folgen, bevor sich ein deutliches Maximum bei $303 \mathrm{~K}$ zeigt. Die zweite industrielle Probe zeigt einen Anstieg zu einem verschmierten Maximum bei ca. $172 \mathrm{~K}$, gefolgt von einem nahezu konstanten strukturlosen Bereich bis 289 K, wo ein Anstieg zu einem Maximum bei $319 \mathrm{~K}$ beginnt.

Abbildung 5.3.8 fasst die Temperaturen der gefundenen Maxima graphisch als Funktion des durch NMR bestimmten MAA-Gehalts zusammen. Dabei sind die Maxima im Bereich des zuerst bei PE beobachteten Tieftemperaturmaximums als $\gamma$-Maximum, die Hauptmaxima als $\alpha$-Maxima und dazwischen liegende Maxima als $\beta$-Maxima bezeichnet, soweit diese deutlich in Erscheinung treten. Die Wahl der Trendlinien ist aufgrund der beschränkten Statistik nur bedingt möglich. So sind die beiden eindeutig gefundenen $\beta$-Maxima der Einfachheit halber mit einer roten Linie verbunden, während bei den Trendlinien der Positionen der $\alpha$ - und $\gamma$ Maxima eine Ausgleichsgerade angedeutet ist. Beide Ausgleichsgeraden scheinen eher eine positive als eine negative Steigung zu besitzen, auch bei Vernachlässigung der industriellen Proben. Ein Zusammenhang von $\gamma$ und $\alpha$ und der Molmasse wurde nicht experimentell bestätigt, wie für $\alpha$ in Abbildung 5.3.9 gezeigt.

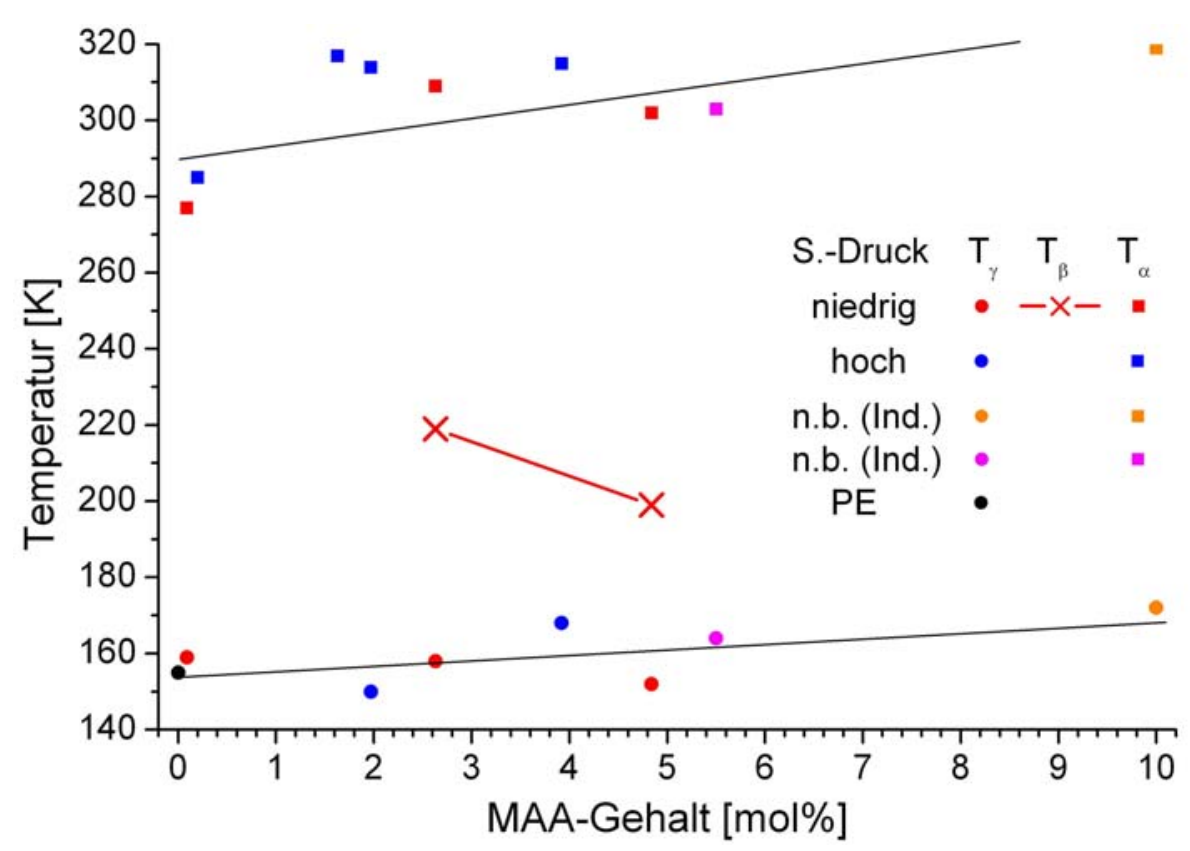

Abb. 5.3.8: Vergleich der charakteristischen Temperaturen in Abhängigkeit des MAAGehalts 


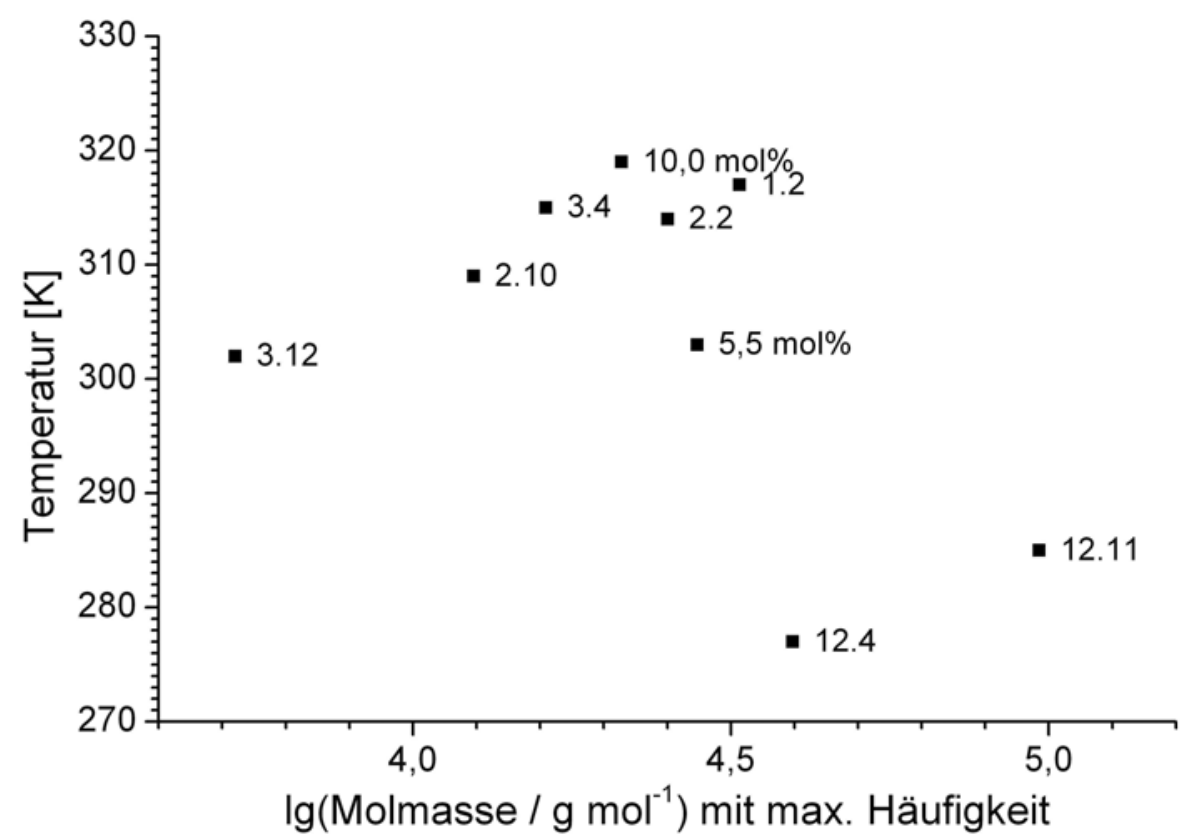

Abb. 5.3.9: Ausschluss des systematischen Zusammenhangs zwischen Temperatur der $\alpha$ Relaxation und der Molmasse in den Copolymerproben 


\section{Diskussion}

Zunächst ist in der Diskussion zu zeigen, dass alle Proben den Qualitätsansprüchen genügen. Dies gilt sowohl für die metallischen Bänder als auch für die synthetisierten Copolymere. Darauf aufbauend werden die mittels DMA und DSC beobachteten Relaxationen und Umwandlungen gedeutet. $\mathrm{Zu}$ deren Identifizierung ist es nötig, die miteinander koppelnden Relaxationen möglichst von einander zu trennen. Aus diesem Grund ist es besonders wichtig zu untersuchen, wie eine Variation der Messparameter die Ergebnisse beeinflusst. Dabei kommt der thermischen und mechanischen Vorgeschichte des Materials eine zentrale Bedeutung zu.

\subsection{Materialeigenschaften der metallischen Bänder}

Die Herstellung der metallischen Bänder aus PdCuSi und $\mathrm{ZrAlCu}$ ist durch Raschabschrecken aus der Schmelze unter Benutzung des „Melt Spinning“ - Verfahrens erfolgt, wie in Kapitel 3.1 beschrieben. Die Ergebnisse der Voruntersuchung der Proben auf Homogenität, Amorphizität und das Kristallisationsverhaltens sind mittels EDX, WAXS und DSC erzielt worden (vgl. Kap. 4.1-3). Handelt es sich um Proben, die zu Beginn der jeweiligen Methode nicht über den in Kapitel 3.1 beschriebenen Abschreckungsprozess hinaus thermisch oder mechanisch vorbehandelt wurden, so werden diese im Folgenden als Ausgangsproben bezeichnet.

\section{Bestimmung der Probenzusammensetzung}

Aus den in den in Kapitel 4 in den Tabellen 4.1.1-2 aufgelisteten, mittels EDX bestimmten Konzentrationen ist ersichtlich, dass die eingewogenen Probenzusammensetzungen gut getroffen worden sind. Dies gilt sowohl für die Ausgangsproben, als auch für solche, die in der DMA zuvor ausgelagert oder kristallisiert worden sind. An Elementen, die nicht in den Proben erwünscht sind, wird nur Sauerstoff gefunden. Die Zielzusammensetzung der $\mathrm{Pd}_{77,5} \mathrm{Cu}_{6} \mathrm{Si}_{16,5}$-Probe stimmt mit dem Mittel aller gemessenen Werte (ohne Sauerstoff) Pd: 77,8 at\%, Cu: 6,2 at\% und Si: 16,0 at \% im Bereich des Fehlers von etwa 1 at $\%$ überein. Die Gesamtzusammensetzung weist einen Sauerstoffgehalt von etwa $5 \mathrm{at} \%$ auf. Gleiches gilt für $\mathrm{Zr}_{65} \mathrm{Al}_{7,5} \mathrm{Cu}_{27,5}$, dessen mittlere Zusammensetzung (ohne Sauerstoff) aus der Ausgangsprobe und der ausgelagerten Probe $\mathrm{zu} \mathrm{Zr}:$ 65,4 at\%, Al: 7,4 at\% und $\mathrm{Cu}: 27,2$ at\% berechnet wird und eine Kontamination mit Sauerstoff von ca. 4 at $\%$ aufweist.

$\mathrm{Ob}$ es sich bei der Sauerstoffkontamination um einen Oberflächeneffekt handelt oder ob dieser Sauerstoffgehalt für das gesamte Probenvolumen repräsentativ ist, kann mit EDX nicht abschließend geklärt werden. So beträgt die Tiefe, aus der noch Röntgenstrahlen detektiert werden, etwa $500 \mathrm{~nm}$. Dies bedeutet, dass ein Großteil des Signals nicht von der Oberfläche stammt.

Eine Auslagerung in der DMA, wie in Abbildung 4.6.1f illustriert, zeigt keinen signifikanten Einfluss auf die Probenzusammensetzung oder die Verteilung der chemischen Elemente auf der Größenskala des Rasterfeldes von ca. 6 x $5 \mu \mathrm{m}$. Gleiches ist bei einer in der DMA kristallisierten PdCuSi-Probe zu sehen. Ein deutlich anderes Verhalten wird bei einer kristallisierten ZrAlCu-Probe an verschiedenen Stellen auf der Schnittfläche, in Punkt- und Rasterfeldmessungen beobachtet. Während der Zr-Anteil sich in der untersuchten Schnittfläche nicht ändert, erscheint der Al-Anteil auf Kosten des Cu-Anteils erhöht. Diese Abweichung der Zusammensetzung wird nur bei dieser Probe beobachtet. Deshalb ist neben einer Fluktuation der chemi- 
schen Zusammensetzung des Bandes auch noch in Erwägung zu ziehen, dass es bei der Kristallisation zu einer kurzreichweitigen Entmischung gekommen sein könnte. Dies rechtfertigt, die abweichende Zusammensetzung der Schnittfläche der kristallisierten ZrAlCu-Probe nicht zur Bestimmung der obigen mittleren Gesamtzusammensetzung hinzuzuziehen.

\section{Überprüfung der Amorphizität}

Das Ergebnis der in Abbildung 4.2.1-2 gezeigten WAXS-Messungen an PdCuSi und $\mathrm{ZrAlCu}$ ist die Bestätigung der Röntgen-Amorphizität der Ausgangsproben. Es werden keine scharfen Maxima beobachtet, die auf kristalline Bereiche in den Proben schließen lassen würden. Die Tatsache, dass in den Messungen viele Probenstreifen von verschiedenen Stellen entlang der Bänder gleichzeitig untersucht werden, lässt eine Verallgemeinerung dieses Schlusses auf die gesamte Länge des Bandes zu.

Das Aufheizen der Proben bei $3 \mathrm{~K} / \mathrm{min}$ bis zu Temperaturen, die im Bereich des Glasübergangs, aber noch unterhalb der Temperatur des Wendepunktes im Phasenwinkel liegen (vgl. Kap. 4.6), hat in Abbildung 4.2.3 keine scharfen Maxima zur Folge. Es zeigen sich also bis zu diesen Temperaturen von $627 \mathrm{~K}$ bei PdCuSi und $665 \mathrm{~K}$ bei $\mathrm{ZrAlCu}$ keine Tendenzen zur Kristallisation. Eine Verschiebung der Position des Maximums kann nicht nachgewiesen werden. Dies liegt daran, dass die Messsignale auf einem hohen Untergrundsignal des zur Befestigung dienenden doppelseitigen Klebebands liegen, wodurch eine Bestimmung der Position der Maxima im Nachkommabereich erschwert wird. Da die Messungen im Gegensatz zu den Abbildungen 4.2.1-2 an einem einzelnen Streifen erfolgen, wird zwangsläufig mehr Klebeband beschienen. Bei $\mathrm{ZrAlCu}$ zeigt sich nach der Auslagerung ein $\mathrm{ZrO}_{2}$-Maximum, dessen Intensität durch ein Schmirgeln der Probe deutlich reduziert wird. Es hat sich also bereits zu diesem Zeitpunkt ein Oberflächenoxid gebildet.

\section{Stabilität in der unterkühlten Schmelze}

Für Messungen im Bereich des Glasübergangs ist die DSC (vgl. Kap. 3.6 und 4.3) als zweite, präparativ relativ unkomplizierte Methode sehr hilfreich. Sie dient zur Eingrenzung der Temperaturbereiche, in denen der Glasübergang und die Kristallisation stattfinden. Diese Effekte werden beeinflusst durch die Wahl der Heizrate und können auch bei konstanter Temperatur zeitabhängig auftreten.

Eine typische DSC-Messung an einer PdCuSi-Ausgangsprobe bei $5 \mathrm{~K} / \mathrm{min}$ ist in Abbildung 4.3.2 (Probe 1) dargestellt. Deutlich zu erkennen ist der charakteristische, endotherme Anstieg im Bereich des Glasübergangs. Eine Mittelung über drei Messungen ergibt einen Onset bei 626(2) K und einen Wendepunkt bei 634(2) K (vgl. Tab. 4.3.1). Die Kristallisation setzt bei ca. $659 \mathrm{~K}$ ein. Bei $\mathrm{Zr}_{65} \mathrm{Al}_{7,5} \mathrm{Cu}_{27,5}$ ist der Bereich zwischen dem Glasübergang und dem Einsetzen der Kristallisation noch größer, wie in [RAM94, S. 29ff] bereits detailliert untersucht wurde. Die darin gefundenen Werte bei ebenfalls $5 \mathrm{~K} / \mathrm{min}$ Heizrate betragen für den Onset des Glasübergangs $633 \mathrm{~K}$, für den Wendepunkt $647 \mathrm{~K}$ und für den Beginn der Kristallisation $694 \mathrm{~K}$. Obgleich diese Messungen mit einer anderen DSC-Kalibrierung (Verwendung von Tiegel und Deckel anstelle zweier Tiegel, vgl. Kap. 3.6) und an per „Splat Quenching“ hergestellten Proben durchgeführt wurden, zeigt dieser Vergleich, dass $\mathrm{ZrAlCu}$ eine stabilere unterkühlte Schmelze besitzt als PdCuSi. 


\subsection{Sekundäre Relaxationen in metallischen Gläsern}

Eines der wesentlichen Ziele dieser Arbeit ist der Nachweis einer sekundären, so genannten langsamen $\beta$-Relaxation in einem fragilen metallischen Glas, im Folgenden im Zusammenhang mit metallischen Gläsern als $\beta$-Relaxation bezeichnet (vgl. Kap. 2.5). Da deren Existenz bereits für starke metallische Systeme gezeigt werden konnte [RSL04], wäre dies ein Indiz für die Universalität einer $\beta$-Relaxation im Rahmen des Glasübergangs amorpher Systeme im Allgemeinen. Da der Glasübergang bei einem fragilen Glas in einem engeren Temperaturfenster abläuft (Abb. 2.4.1), besteht die Hoffnung, dass sich dadurch die $\alpha$ - und $\beta$-Relaxation in einem fragilen Glas leichter voneinander trennen lassen, zumal die Untersuchungen bei tieferen Frequenzen stattfinden.

\section{ZrAICu}

Erste Indizien für die Existenz einer $\beta$-Relaxation wurden, wie in [RÖS04, RSL04] beschrieben, mittels mechanischer Spektroskopie bei $5,44 \mathrm{kHz}$ an $\mathrm{ZrAlCu}$-Filmen gefunden. Die effektive Heizrate betrug dabei ca. 0,2 K/min. Bei hohen Frequenzen sind die Relaxationen zu hohen Temperaturen verschoben (vgl. Abb. 6.2.1 und Abb. 2.5.1). Die $\alpha$-Relaxation lässt sich mit dieser Methode nicht ausmessen, weil vor deren Erreichen die Kristallisation einsetzt. Vorteilhaft ist dagegen die sehr niedrige Grunddämpfung, die eine hohe Empfindlichkeit im Bereich des nahezu konstanten Verlusts (NCL), oder sub- $T_{\mathrm{G}}$-Bereichs erlaubt. Eine Illustration des gemessenen Verlusts im Bereich des NCL und der $\beta$-Relaxation zeigt Abbildung 6.2.2. Die $\beta$-Relaxation tritt dabei nur in Form einer Tieftemperaturflanke, eines so genannten ,excess wing“, des mit Hilfe von Literaturdaten angenäherten $\alpha$-Relaxationsmaximums auf.

Bei tieferen Frequenzen, wie sie bei der DMA verwendet werden, erscheinen die Relaxationsmaxima bei tieferen Temperaturen. Es ist somit ein größerer Teil des Relaxationsspektrums zugänglich, in dem noch keine Kristallisation einsetzt. Eine typische Messung mittels DMA an $\mathrm{ZrAlCu}$ bei $1 \mathrm{~Hz}$ Frequenz, $3 \mathrm{~K} / \mathrm{min}$ Heizrate und relativ kleinen Kräften von $200 \mathrm{mN}$ statisch und $150 \mathrm{mN}$ dynamisch zeigt Abbildung 4.5.1. Zur Anpassung der Daten werden dabei dieselben wie in [RÖS04] verwendeten Werte für den Stärkeparameter $D_{\mathrm{VFT}}$ und die Vogel-Fulcher-Tammann-Temperatur $T_{\mathrm{VFT}}$ genutzt und ebenfalls eine ColeDavidson-Fitfunktion $(\alpha=1)$ angepasst. Der Fit wird an dem hervorgehobenen Datenbereich durchgeführt, welcher am nützlichsten zur Bestimmung der Anfangswerte des Speicher$\left(E_{0}=\Delta E\right)$ und Verlustmoduls $\left(E_{\mathrm{NCL}}\right)$ ist, bzw. sich im Bereich der $\alpha$-Relaxation befindet. Den Satz der am besten passenden Fitparameter fasst Tabelle 4.5.1 zusammen. Die nicht trivialen, freien Fitparameter sind dabei nur $\tau_{0}$ und $\gamma . \tau_{0}$ entspricht der kürzesten Zeit, in der im Material eine Relaxation stattfinden kann. Mit $\gamma$ lässt sich eine asymmetrische Verbreiterung der $\alpha$ Relaxation auf der Tieftemperaturflanke erreichen. Die Anpassung der Höhe des Maximums gelingt aber nicht, weswegen der Verlust nicht zum Fit im Bereich der $\alpha$-Relaxation genutzt wird. Die minimale Relaxationszeit $\tau_{0}$ erscheint dabei mit $4 \cdot 10^{-14} \mathrm{~s}$ als sehr klein, auch wenn sie im Intervall zwischen den in [RÖS04] und [RSL04] gefundenen Werten für diesen Fitparameter liegen. 


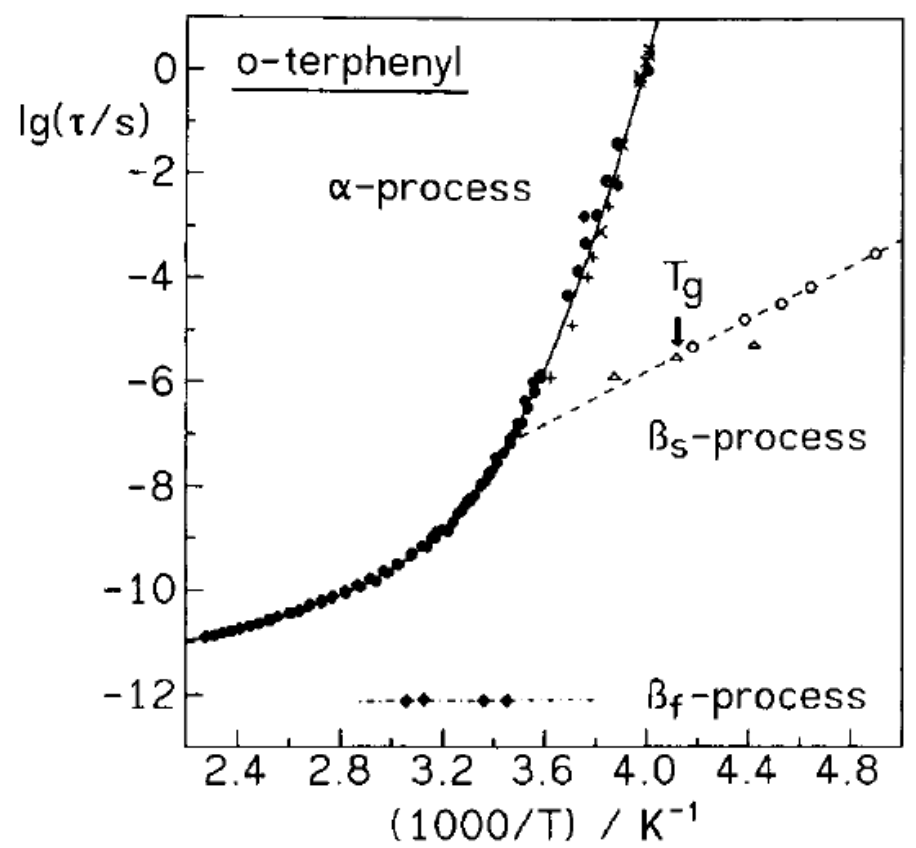

Abb. 6.2.1: Temperaturabhängigkeit der Relaxationszeiten von $\alpha$-, slow $\beta$ - und fast $\beta$ Relaxation von Orthoterphenyl [EAN96], eingetragen sind die Resonanzen $\omega=\tau^{-1}$ (vgl. Kap.2, Gleichung (18))

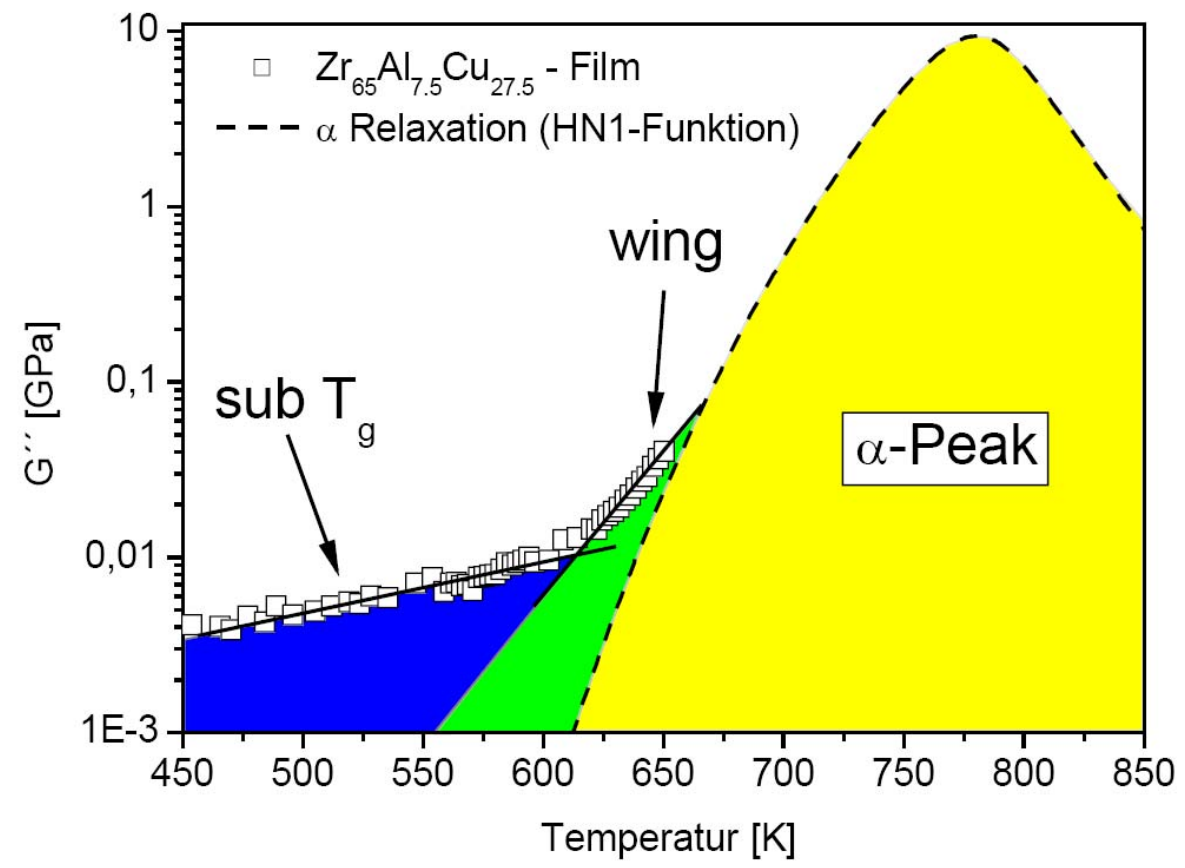

Abb. 6.2.2: Erstes Indiz für die Existenz eines ,excess wing“ aus [RÖS04]

Aufgrund dieser Schwierigkeiten erfolgen alle folgenden Anpassungen der elastischen Moduln mit einer Cole-Cole-Funktion mit festem $\tau_{0}$, wie in Abbildung 4.5.2 an derselben Messkurve angewendet. Als kleinste Zeit für eine mechanische Relaxation wird dabei $\tau_{0}=10^{-12} \mathrm{~s}$ fest gewählt. Diese Wahl ist sinnvoll, da der Boson-Peak in diesem Bereich sein Maximum besitzt (vgl. Abb. 2.5.1, [CSB04, ANG04, MWB99]). Die Benutzung der Cole-Cole-Funktion 
führt zu einer symmetrischen Verbreiterung. Dies hat zur Folge, dass sich die Höhe des Verlustmaximums reduziert und somit ein Teil der gemessenen Werte des Verlustmoduls im Bereich der $\alpha$-Relaxation zur Anpassung mitgenutzt werden kann. Dies beruht auf der Tatsache, dass Speicher- und Verlustmodul durch die Kramers-Kronig-Relation [PRI99] verbunden sind: Fällt der Speichermodul auf einen tieferen Wert ab, so muss im Verlust ein Maximum beobachtet werden. Um eine gegebene Änderung des Speichermoduls zu erreichen, muss das Verlustmaximum über eine gewisse Fläche unter der Kurve verfügen. Aus diesem Grund ist eine symmetrische Verbreiterung zu beiden Seiten effektiver als eine asymmetrische, die nur auf der Tieftemperaturflanke zu einer Verbreiterung führt.

Die Hochtemperaturflanke wird gänzlich außer Acht gelassen, da in diesem Temperaturbereich ein Einsetzen der Kristallisation nicht mehr ausgeschlossen werden kann. Dieser Effekt beendet vorzeitig den Abfall des Speichermoduls und senkt den Verlust. Das Maximum erscheint zu kleineren Temperaturen verschoben. Ein gutes Kriterium für die Beurteilung, bis zu welcher Temperatur die Daten verlässlich sind, bietet die Änderung der statischen Dehnung, wie in der folgenden Abbildung 6.2.3 aufgetragen. Sie ist im Wesentlichen invers proportional zur Viskosität (Gleichung (5)), wobei die effektive Spannung mit sinkender Querschnittsfläche des Bandes zunimmt.

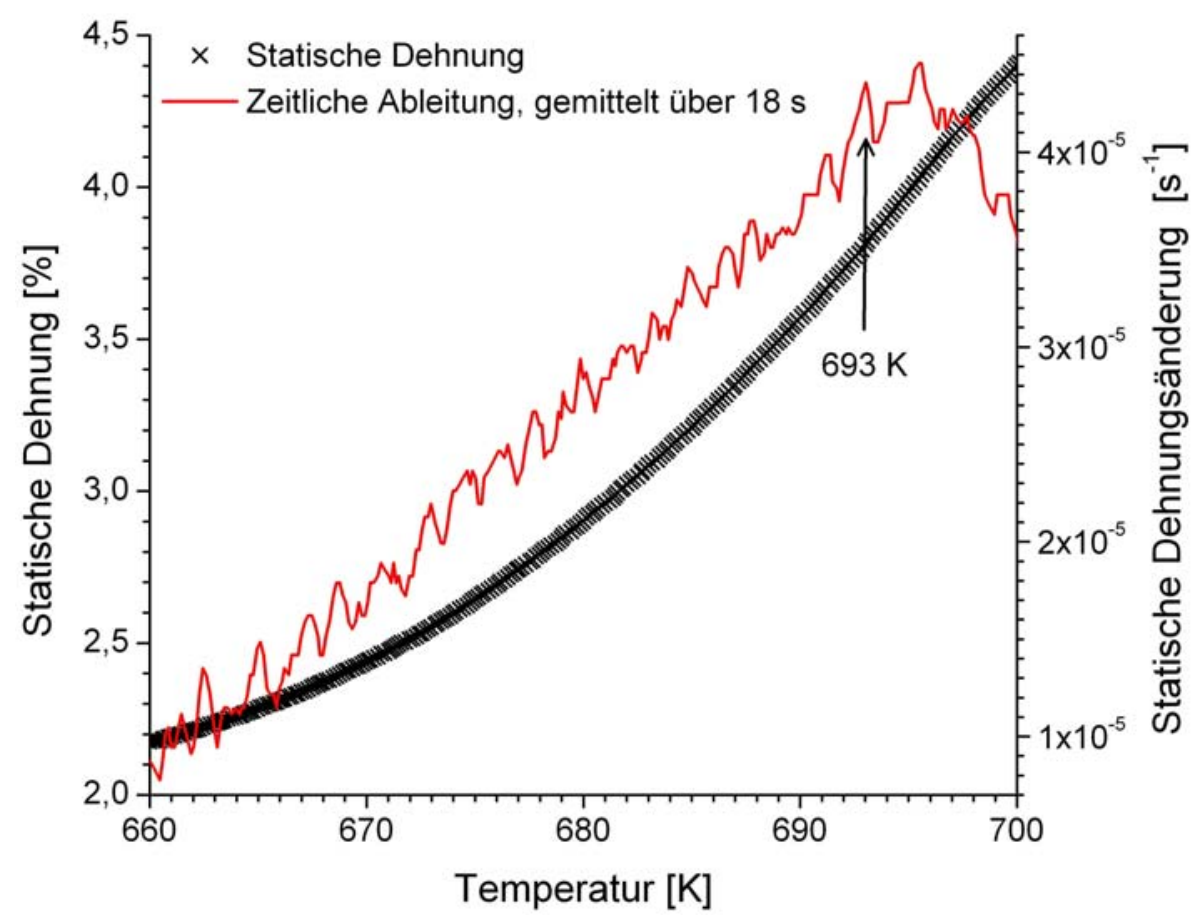

Abb. 6.2.3: Kriterium für den Beginn der Kristallisation (Messrate: 20 Datenpunkte/s) zur Messung an $\mathrm{ZrAlCu}$ in Abb. 4.5.1

Der Verlauf der Dehnungsänderung ohne Kristallisation sollte als Funktion der Temperatur streng konvex verlaufen. Dies ist bis $693 \mathrm{~K}$ der Fall. Der Verlust (vgl. Abb. 4.5.1) durchläuft bei dieser Temperatur ein Maximum, der Wendepunkt des Verlusts liegt aber bereits bei 677 K. Der Speichermodul weist einen Knick bei $695 \mathrm{~K}$ auf, und der nicht gezeigte Tangens des Phasenwinkels durchläuft ebenfalls bei $693 \mathrm{~K}$ einen Wendepunkt. Dabei reagiert das viskose Fließen und somit die Dehnungsänderung sensibler auf ein Einsetzen der Kristallisation 
als der Verlustmodul. Dies begründet sich dadurch, dass am viskosen Fließen größere, sich kooperativ umlagernde Schertransformationszonen (STZ) teilnehmen als jene, die durch Spektroskopie geschert werden [JS05].

Das Einmünden des Verlustmoduls in ein Maximum im Bereich seines Wendepunktes in Abbildung 4.5.1f ist also verlässlich, während die genaue Position des Maximums bereits durch eine einsetzende Kristallisation verfälscht sein könnte. Abschließend bleibt zum Fitversuch an $\mathrm{ZrAlCu} \mathrm{zu}$ bemerken, dass auch die Anpassung mit einer Cole-Cole-Funktion nicht zufrieden stellend möglich ist.

\section{PdCuSi}

Den Unterschied zwischen $\mathrm{ZrAlCu}$ und $\mathrm{PdCuSi}$ unter identischen Versuchsbedingungen zeigt Abbildung 4.5.3. Dabei sind die beiden Spektren so zueinander verschoben, dass die horizontalen Positionen der Verlustmaxima aufeinander fallen. Der fragilere Charakter des PdCuSi wird hier offenbar: Sowohl der Abfall des Speichermoduls als auch der Anstieg zum Verlustmaximum sind deutlich ausgeprägter. Außerdem kommt es aufgrund des raschen Abfalls der Viskosität zu einem Auseinanderfließen der Probe, während bei $\mathrm{ZrAlCu}$ die Kristallisation zu einer Wiederverfestigung des Materials führt. Trotz der schärferen $\alpha$-Relaxation von PdCuSi ist auf der Tieftemperaturflanke keine klare $\beta$-Relaxation zu erkennen.

Bei ebenfalls $1 \mathrm{~Hz}$ Frequenz, aber $5 \mathrm{~K} / \mathrm{min}$ Heizrate und kleineren Kräften von $250 \mathrm{mN}$ statisch und $200 \mathrm{mN}$ dynamisch, zeigt die Messung der elastischen Moduln in Abbildung 4.4.2 deutlichere Effekte unterhalb der $\alpha$-Relaxation (eine Begründung dafür liefern die folgenden Kapitel 6.3 und 6.4). Insbesondere während der vierminütigen Haltephase bei fester SollTemperatur von $573 \mathrm{~K}$ zeigt sich ein Anstieg des Speichermoduls. Dieser geht mit einem Anstieg im Verlustmodul einher, wobei die logarithmische Auftragung etwas darüber hinwegtäuscht, dass die Änderung des Speichermoduls während der Haltephase 38 \% beträgt, wogegen der Verlustmodul um weniger als $9 \%$ ansteigt. Dies zeigt sich auch in den Rohdaten in Abbildung 4.4.1, in welcher die Amplitude der Schwingung deutlicher sinkt als der Phasenwinkel. Auch während der Messung ist noch ein leichter Anstieg des Speichermoduls bis etwa $606 \mathrm{~K}$ zu beobachten, während der Verlustmodul in diesem Bereich nahezu konstant ist.

Unter der Annahme, dass in diesem nahezu konstanten Bereich die Anfangseffekte (im Folgenden genauer behandelt in Kap. 6.5) bereits abgeklungen sind, so ist es sinnvoll, diesen Bereich als NCL anzusehen und zum Anpassen des Fitparameters $E_{\mathrm{NCL}}$ zu nutzen. Der in Abbildung 4.4.3 gezeigte Cole-Cole-Fit zeigt eine bessere Anpassung der gemessenen Daten als die zuvor diskutierten Messungen an $\mathrm{ZrAlCu}$. Er ist aber insbesondere die Steigung des Verlustmoduls betreffend nicht zufrieden stellend. Eine größere Verbreiterung der Relaxation durch einen kleineren Wert für $\alpha$ würde zu einer besseren Anpassung sowohl der Höhe des Verlustmaximums, als auch dessen Steigung auf der Tieftemperaturflanke führen. Dies hätte aber auch zur Folge, dass die Anpassung an den Speichermodul dessen Verlauf im zum Fit benutzten Bereich schneiden würde. Es ist aber nicht einzusehen, warum die gemessenen Werte des Speichermoduls schneller abfallen sollten als erwartet. Ein Einsetzen der Kristallisation hätte den gegenteiligen Effekt und kann somit nicht zur Deutung bemüht werden.

Folglich bleibt nur die Erklärung, dass die $\alpha$-Relaxation alleine nicht zur Deutung der beobachteten Effekte genügt. Angenommen, im Bereich der Tieftemperaturflanke ist eine zusätz- 
liche Relaxation aktiv, die zwar nur zu einem flachen Maximum im Verlustmodul führt, sich aber über einen breiten Temperaturbereich erstreckt, so gelingt eine deutlich bessere Anpassung des Verlustmaximums im Bereich des zweiten Anstiegs, wie in Abbildung 4.4.4 angewendet. $E_{0}=\Delta E$ wurde dazu um ca. $3,5 \mathrm{GPa}$ auf $24 \mathrm{GPa}$ reduziert, woraus eine gute Anpassung der Höhe des Verlustmoduls resultiert. Aus dieser Differenz eine Relaxationsstärke der sekundären Relaxation zu berechnen ist nicht ohne Weiteres möglich, da nicht geklärt ist, in welcher Weise die sich überlagernden Relaxationen miteinander koppeln [ARC96]. Der Verlauf des Speichermoduls kann nun erst im Bereich der $\alpha$-Relaxation angepasst werden. Im Anfangsbereich resultiert der langsame Abfall nicht aus der $\alpha$-Relaxation, da der Verlauf des Fits (der nur NCL und $\alpha$ berücksichtigt) in diesem Bereich ein Plateau aufweist. Die Steigung des Fits ist im Bereich des Wendepunkts des Speichermoduls nur unwesentlich steiler als die gemessenen Daten. Der Effekt der einsetzenden Kristallisation macht sich im Speichermodul erst ab dessen Wendepunkt bei 671(2) K deutlich bemerkbar. In kalorimetrischen Messungen wurde ein Onset der Kristallisation von ca. $659 \mathrm{~K}$ ermittelt, was sehr gut mit der Temperatur des Wendepunkts in der Änderung der statischen Dehnung bei 660(2) K übereinstimmt (vgl. Abb. 6.2.3). Aus diesen Abschätzungen resultiert die Wahl der zum Fit genutzten, in Abbildung 4.4.4 hervorgehobenen, Datenpunkte.

Wie fragil PdCuSi im Vergleich zu ZrAlCu ist, lässt sich aus dem angepassten Stärkeparameter $D_{\mathrm{VFT}}$ ersehen. Er ist über Gleichung (9) mit der Fragilität $m$ verknüpft. So ist für PdCuSi eine Fragilität von $m=76,8$ gefunden worden; für $\mathrm{ZrAlCu}$ wurden Werte im Bereich von $m=36,4-38,4$ in Messungen der Temperaturabhängigkeit der Viskosität ermittelt [IRÖ04, RAM94, RÖS04]. Durch ein vorheriges Auslagern von PdCuSi in älteren Arbeiten ist eine Verschiebung der Fragilität von $m=71,4$ zu 52,8 beobachtet worden [TS85, CG72].

Für ZrAlCu wird für $m=38,4$ analog zu [RÖS04] ein $D_{\mathrm{VFT}}=26,3$ erwartet, das in Abbildung 4.5.1 zum Anpassen der Daten genutzt wurde. Dieser Fit ist aber, wie bereits erwähnt, wenig befriedigend. Der Cole-Cole-Fit (Abb. 4.5.2) tendiert zu einem kleineren Stärkeparameter, also einer höheren Fragilität. Für PdCuSi wäre aus $m=76,8$ [IRÖ04] ein $D_{\mathrm{VFT}}=9,7$ zu erwarten. Die gefundenen Stärkeparameter in Abbildung 4.4.3f liegen noch darunter bei 5,6 - 7,8, dies entspricht einer größeren Fragilität von $m=92-121$. Diese Werte erscheinen im Vergleich mit der Literatur sehr hoch. Eine mögliche Erklärung ist, dass sie dynamisch ermittelt wurden. Die Frequenz ist dadurch deutlich höher als bei quasi-statischen Messungen der Viskosität. Da bei höheren Frequenzen die Resonanzbedingung (Gleichung (18)) erst bei höheren Temperaturen erreicht ist, wo $\alpha$ - und $\beta$-Relaxation dichter zusammen liegen und somit der Übergang schärfer wird, verhält sich das System fragiler (vgl. Abb. 2.4.1 und 6.2.1). Darüber hinaus ist $D_{\mathrm{VFT}}$ nicht unabhängig von der Wahl von $\tau_{0}=10^{-12} \mathrm{~s}$. Aus der Veränderung dieses Wertes um eine Zehnerpotenz resultiert bei Temperaturen von $130 \mathrm{~K}$ über $T_{\mathrm{VFT}}$ eine Änderung in $D_{\mathrm{VFT}}$ von 0,6 .

Der durch Herabsetzung von $E_{0}=\Delta E$ erreichte, den NCL und die $\alpha$-Relaxation berücksichtigende Fit an den Verlustmodul ist in Abbildung 6.2.4 noch einmal vergrößert dargestellt (vgl. Abb. 4.4.4). Dabei wurden die gefundenen Relaxationen dem Beispiel von [RÖS04] in Abbildung 6.2.2 folgend farblich illustriert. Es ist deutlich zu erkennen, dass ein nur auf NCL und $\alpha$-Relaxation beruhender Fit nicht ausreicht, die Messdaten zu beschreiben. Zwischen $654 \mathrm{~K}$ und dem Einsetzen der Kristallisation, sowie unterhalb von $613 \mathrm{~K}$ gelingt dies gut, nicht je- 
doch im Temperaturintervall dazwischen. Deutlich zu erkennen ist ein „excess wing“, der von einem vom $\alpha$-Maximum überdeckten, sekundären Relaxationsprozess herrührt. Dies ist ein deutliches Indiz für die Existenz einer langsamen $\beta$-Relaxation in einem fragilen metallischen Glas. Da dieselben Beobachtungen auch am starken metallischen Glas ZrAlCu (vgl. Abb. 6.2.2 aus [RÖS04] und Kapitel 4.5) gemacht werden können und in metallischen Gläsern, die als System harter Kugeln keine weiteren Ursachen für Relaxationen erkennen lassen, scheint es sich bei der langsamen $\beta$-Relaxation um ein für den Glasübergang universelles Phänomen zu handeln.

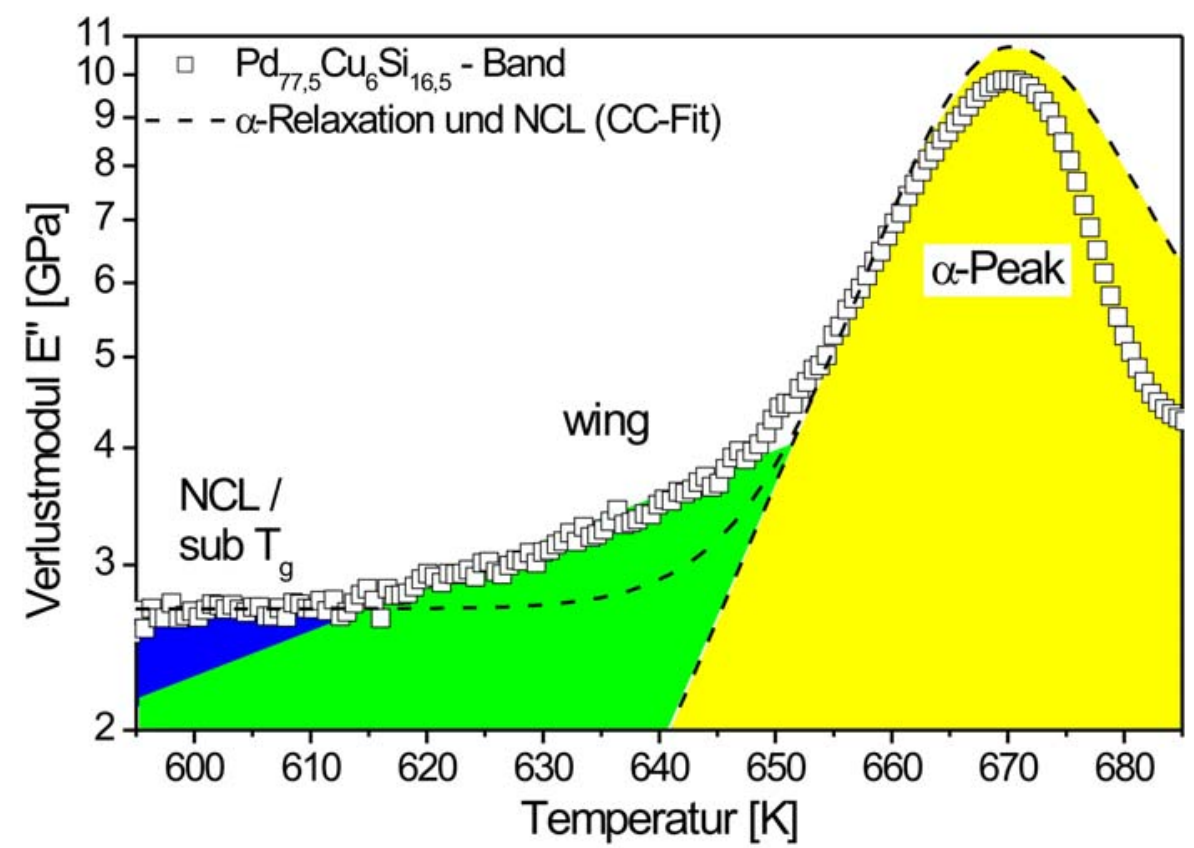

Abb. 6.2.4: Separation des $\beta$-Prozesses aus dem Verlustspektrum von PdCuSi (vgl. Abb. 4.4.4)

\subsection{Raten-, Frequenz- und Kraftabhängigkeit der beobachteten Phä- nomene}

Ziel dieses Unterkapitels ist es zu beleuchten, in wieweit die Wahl der Messparameter die Form und die Lage der beobachteten Relaxationsprozesse verändert. Die Abbildungen 4.4.5-8 zeigen dazu Messungen des Temperaturverlaufs des Tangens des Phasenwinkels $(\tan \delta$ ) an PdCuSi, bei denen die Messparamter Heizrate, Frequenz und Kraft systematisch verändert werden.

Bei der Variation der Heizrate von $1 \mathrm{~K} / \mathrm{min}$ bis $40 \mathrm{~K} / \mathrm{min}$ in Abbildung 4.4.5 bei dynamischen Messungen mit $1 \mathrm{~Hz}$ und kleinen Kräften von $100 \mathrm{mN}$ statisch und $50 \mathrm{mN}$ dynamisch ist eine Verschiebung des Glasübergangs (im Folgenden Onset von tan $\delta$ ) zu höheren Temperaturen zu erkennen (vgl. Tabelle 4.4.3). Dieser Effekt entspricht den Erwartungen, muss aber dadurch abgeschwächt werden, dass die Heizrate sich auch signifikant in der Kalibrierung der Temperatur niederschlägt. Alle in Kapitel 4.4 gezeigten Messungen sind auf die am häufigsten verwendete Heizrate von $5 \mathrm{~K} / \mathrm{min}$ mit einem Zink- und Zinn-Standard kalibriert, wodurch die Abbildungen 4.4.5 und 4.4.8 in Bezug auf die tatsächliche Temperatur korrigiert werden müssen. In [RAM94] wird diese Korrektur durch das Subtrahieren einer konstanten, durch 
Messungen an einem Zink-Standard gewonnenen Verschiebung erreicht, wie in Tabelle 4.4.3 durchgeführt. Nach der Korrektur ist bei kleinen Heizraten von $1 \mathrm{~K} / \mathrm{min}$ und $5 \mathrm{~K} / \mathrm{min}$ keine signifikante Verschiebung festzustellen, wie auch in [RAM94] an $\mathrm{ZrAlCu}$ gemessen. Diese zeigt sich erst beim Übergang zu Heizraten von $20 \mathrm{~K} / \mathrm{min}$ oder $40 \mathrm{~K} / \mathrm{min}$. Bei $1 \mathrm{~K} / \mathrm{min}$ bleibt der Probe genügend Zeit um zu kristallisieren, bevor die Viskosität soweit abfällt, dass es zu einem Auseinanderfließen der Probe kommt.

Die Abhängigkeit von Frequenz und Amplitude zeigt Abbildung 4.4.6. Eine Verschiebung des Onsets mit der Frequenz ist deutlich zu sehen, wie in Tabelle 4.4.4 zusammengefasst (vgl. Abb. 6.2.1). Eine signifikante Abhängigkeit des Onsets von der Höhe der Amplitude, welche direkt mit der anliegenden Kraft verknüpft ist, kann nicht festgestellt werden. Es fällt jedoch eine schwache Systematik auf, wonach für $\tan \delta>0,4$ die Kurvenverläufe mit höheren Amplituden bzw. Kräften bei gleicher Frequenz zu niedrigeren Temperaturen verschoben sind. Eine mögliche Erklärung für diese Anzeichen eines schnelleren Anstiegs bei höheren Kräften ist das Auftreten von Nicht-Newton'schem Fließen. Dabei wird die Viskosität selbst abhängig von der Scherrate. Die Scherrate kann nicht fest vorgegeben werden. Sie ist aber höher, wenn die Spannung erhöht wird oder eine größere Amplitude eingeregelt werden soll. Hinzu kommt die Möglichkeit der Bildung von Einschnürungen. Dies scheint zumindest bei der Messung bei $10 \mathrm{~Hz}$ und $6 \mu \mathrm{m}$ Amplitude nicht unerheblich, da bei $660 \mathrm{~K}$ die statische Dehnung bereits $42 \%$ beträgt und darüber rapide ansteigt. Gleiches gilt für die Messung bei $10 \mathrm{~Hz}$ und 3,4 $\mu \mathrm{m}$ Amplitude. Bei allen anderen Messungen beträgt die statische Dehnung maximal $10 \%$ im gezeigten Bereich.

Diese Abhängigkeit der Viskosität von den verwendeten Kräften bei vergleichbaren Querschnittsflächen zeigen die Abbildungen 4.4.7-8, wobei letztere, wie bereits erwähnt, in ihrer Temperatur um 4,5 K zu kleineren Werten korrigiert werden müsste. Der erste Abfall rührt daher, dass sich erst eine stabile Heizrate einstellen muss. Die lineare Wärmeausdehnung multipliziert mit der Heizrate ergibt die Korrektur der zeitlichen Änderung der Probenlänge aufgrund viskosen Fließens. Durch die Normierung auf die Probenlänge ergibt sich daraus die zeitliche Dehnungsänderung, welche über Gleichung (5) mit der Viskosität verknüpft ist.

Die Tatsache, dass die Viskosität von der Spannung abhängt, zeigen Abbildungen 4.4.7-8 deutlich. Diese Abhängigkeit ist dabei sowohl reproduzierbar (Abb. 4.4.8), als auch systematisch, allerdings entgegengesetzt der ersten Erwartung, dass die Viskosität mit steigender Spannung abnehmen sollte (,shear thinning“, [LRJ03, DJ05, DHT06]). Dieser gegenteilige Effekt, dass bei einer höheren Spannung eine niedrigere Viskosität beobachtet wird, ist darin begründet, dass die Messung nicht bei konstanter Temperatur erfolgt. Es handelt sich also nicht um die Gleichgewichtsviskosität $\eta_{\infty}$ (Gleichung (12)) bei der aktuellen Temperatur. Der Effekt, dass die Gleichgewichtsviskosität umso schneller erreicht wird, je höher die Spannung ist, wurde bereits bei Messungen bei konstanter Temperatur in [IRÖ04, S. 60] beobachtet. Im Bereich des Glasübergangs streben die Kurven wieder zusammen. Der Hauptgrund für das Entstehen eines Schnittpunktes ist aber das Einsetzen der Kristallisation, die bei Proben, an denen mit kleinen Kräften gezogen wird, zu einem Wiederanstieg der Viskosität führt, während hohe Kräfte zu einem Auseinanderfließen der Probe führen. 


\subsection{Verhalten auf großen Temperaturskalen und gezieltes Altern}

In den vorangegangenen Unterkapiteln 6.2 und 6.3 wird bereits an mehreren Stellen offenbar, was es heißt, an einem System zu messen, dessen Zustand weit entfernt von einem stabilen Gleichgewicht ist. Dies zeigt sich sowohl in den in Kapitel 6.2 erwähnten Anfangseffekten vor dem Einmünden des Verlusts in einen NCL-Bereich, als auch im oben diskutierten Umgekehrt-Nicht-Newton'schen Effekt. Aus diesem Grund soll in diesem Kapitel das Verhalten von metallischen Gläsern auf großen Temperaturskalen und die Auswirkung von thermischen und mechanischen Vorbehandlungen sowie die Variation der Heizrate genauer untersucht werden.

Die großen Temperaturskalen werden durch ein Herabsetzen der Anfangstemperatur mittels Stickstoffkühlung erreicht. Bei Temperaturen um Zimmertemperatur und darunter ist eine Zinn- und Zink-Kalibrierung nicht mehr zweckmäßig, weshalb für Heizraten von $3 \mathrm{~K} / \mathrm{min}$ und $20 \mathrm{~K} /$ min Kalibrierungen mittels eines zweiten Thermoelements am Probenort (vgl. Abb. 3.10.2) durchgeführt wurden.

Die Wahl der Messparameter begründet sich auf der folgenden Überlegung: Um das Alterungsverhalten untersuchen zu können, müssen als Referenz auch Proben gemessen werden, die noch so nah wie möglich am Zustand der Ausgangsproben sind. Eine Grundvoraussetzung dafür ist eine hohe Heizrate, damit die Probe möglichst kurz in einem Temperaturbereich verweilt, in dem Veränderungen auftreten. Auf der anderen Seite ist eine möglichst kleine Messfrequenz sinnvoll, da dann die $\alpha$-Relaxation zu niedrigen Temperaturen verschoben ist. $20 \mathrm{~K} / \mathrm{min}$ und $1 \mathrm{~Hz}$ stellen einen gut mit der DMA realisierbaren Kompromiss dar, weil auf diese Weise pro Kelvin Temperaturunterschied das Messsignal über drei Schwingungszyklen gemittelt werden kann. Die Kräfte sollten möglichst klein sein, wenn, wie oben beschrieben (vgl. Abb. 4.4.7f), die Viskosität sich möglichst weit weg von einem thermischen Gleichgewichtswert befinden soll (Gleichung (12)). Andererseits sollte die Kraft groß genug sein, um eine einfach zu messende Amplitude zu gewährleisten, wie es bei $150 \mathrm{mN}$ dynamischer Kraft gerade noch der Fall ist. Höhere Heizraten, kleinere Frequenzen und niedrigere Spannungen wären wünschenswert, würden bei der DMA aber zwangsläufig zu einem nicht akzeptablen Verhältnis von Signal und Rauschen führen. Die zusätzlichen Untersuchungen bei $3 \mathrm{~K} / \mathrm{min}$ sollen zeigen, ob bereits bei dieser niedrigeren Heizrate deutliche Veränderungen des Materials stattfinden können.

Die definierte Herstellung von gealterten Proben ist in Kapitel 4.6 beschrieben und sei hier nur kurz wiederholt: PdCuSi- und ZrAlCu-Ausgangsproben (rasch abgeschreckt und nicht weiter thermisch oder mechanisch vorbehandelt, vgl. Kap. 3.1) werden bei anliegenden Kräften mit der stets gleichgehaltenen Frequenz zunächst aus Gründen der Zeitersparnis mit $20 \mathrm{~K} / \mathrm{min}$ geheizt. Anschließend erfolgt das weitere Heizen mit $3 \mathrm{~K} / \mathrm{min}$, bis sich deutliche Effekte der einsetzenden $\alpha$-Relaxation zeigen, um eine deutliche Veränderung des Materials zu erreichen. Dabei können erstaunliche Unterschiede zwischen PdCuSi und ZrAlCu im Vergleich mit einem zweiten Aufheizen derselben Probe bei $20 \mathrm{~K} / \mathrm{min}$ in Abbildung 4.6.1f entdeckt werden: Bei PdCuSi ändert sich der Speichermodul beim Alterungsschritt nicht wesentlich, während der Verlustmodul auf $8 \mathrm{GPa}$ ansteigt. Bei $\mathrm{ZrAlCu}$ ist dagegen ab etwa $420 \mathrm{~K}$ ein deutlicher Anstieg des Speichermoduls um $20 \% \mathrm{zu}$ beobachten, gleichzeitig steigt der Verlustmodul auf nur $4 \mathrm{GPa}$. Beim Abkühlen (nicht gezeigt) und anschließenden Aufheizen 
derselben PdCuSi-Probe zeigt sich auch eine deutliche Erhöhung des Speichermoduls um ebenfalls ca. $20 \%$. Ab $610 \mathrm{~K}$ verläuft die Messung des Speichermoduls der gealterten $\mathrm{ZrAlCu}-\mathrm{Probe}$ im Wesentlichen auf der Linie der während des Alterns gewonnenen Daten (in Kap. 4.6 beschriebene Temperaturkalibrierung beachten). Bei den gegebenen Messparametern hat der Speichermodul beim ersten Durchlauf ab dieser Temperatur einen Sättigungswert erreicht.

Der Vergleich des Verlaufs des Verlustmoduls zwischen gealterten Proben und Ausgangsproben zeigt bei $3 \mathrm{~K} / \mathrm{min}$ in Abb. 4.6 .3 praktisch keine Unterschiede zwischen der gealterten $\mathrm{ZrAlCu}$ - Probe und einer Referenzmessung. Das Altern hat die Kurvenform der PdCuSiProbe nicht verändert, diese aber um 0,7 GPa abgesenkt und um ca. 1,7 K zu höheren Temperaturen verschoben. Dies scheint zunächst den Erwartungen zu widersprechen (vgl. Kap. 2.3). Es liegt aber vermutlich daran, dass Altern und $\alpha$-Relaxation gleichzeitig aktiv sind und miteinander koppeln. Bei der gealterten Probe liegt hauptsächlich $\alpha$ vor, das bei höheren Temperaturen liegt als das Altern. Bei $20 \mathrm{~K} / \mathrm{min}$ zeigt sich bei PdCuSi dasselbe Phänomen, nicht aber bei $\mathrm{ZrAlCu}$ : Bei der Ausgangsprobe ist ein deutlich flacherer Anstieg zu beobachten, verglichen mit der gealterten Probe. Konnten $\alpha$-und $\beta$-Relaxation bei PdCuSi bei $1 \mathrm{~Hz}$ Frequenz und Heizraten von $3 \mathrm{~K} / \mathrm{min}$ und $20 \mathrm{~K} / \mathrm{min}$ (vgl. Abb. 4.6.1) nicht deutlich voneinander getrennt gemessen werden, so ist dies bei einer veränderten Wahl der Messparameter möglich.

Bei $5 \mathrm{~Hz}$ und $10 \mathrm{~K} / \mathrm{min}$ ist in Abbildung 4.6 .5 bei unveränderten Kräften beim ersten Aufheizen zunächst ein deutlicher Anstieg des Speichermoduls um mehr als $30 \%$ zu beobachten. Dieser setzt bereits unter $460 \mathrm{~K}$ ein und wächst beim Abkühlen weiter an. Beim zweiten Aufheizen ist das Material bei Zimmertemperatur um $60 \%$ steifer. Der Kurvenverlauf dieser Messung an PdCuSi besitzt jetzt große Ähnlichkeit in der Form zu ZrAlCu in Abbildung 4.6.2. Der Verlust zeigt nun einen deutlichen Übergang vom NCL zur $\alpha$-Relaxation, welche eine ausgeprägte Tieftemperaturflanke besitzt. Ob der Anstieg beim ersten Aufheizen ab ca. $280 \mathrm{~K}$ bereits den Beginn des Alterungsprozesses beschreibt, ist aufgrund des hohen Rauschens nicht sicher zu sagen. Der zweite Anstieg ab ca. $488 \mathrm{~K}$ ist dagegen deutlich. Im Unterschied zum ersten Aufheizen zeigt der zweite Durchlauf nach einem anfangs geringfügigem Anstieg einen breiten Bereich des NCL, etwa zwischen $220 \mathrm{~K}$ und $510 \mathrm{~K}$. Der darauf folgende Anstieg besitzt deutlich zwei Onsets bei besagten $510 \mathrm{~K}$ und bei ca. $622 \mathrm{~K}$. Dies ist ein deutliches Zeichen für eine $\beta$-Relaxation, wie in Abbildung 6.2.4 illustriert.

\subsection{Deutungsversuch der Relaxationsspektren}

\section{Annahmen und Voraussetzungen}

Für die konsistente Deutung aller in den Messungen beobachteten Relaxationen werden die folgenden Annahmen benötigt, bzw. es gilt als Voraussetzung:

Es gibt (mindestens) zwei Relaxationsprozesse, $\alpha$ und $\beta$, mit dazugehörigen Relaxationszeiten.

Jede DMA-Messung besitzt zwei charakteristische Zeitskalen bzw. Frequenzen, beruhend auf Spektroskopie und Heizrate. 
Kommt es zur Resonanz zwischen einer der Relaxationszeiten und einer der charakteristischen Zeitskalen bzw. Frequenzen, so zeigt sich ein Maximum im Verlust, begleitet von einer Änderung des Speichermoduls.

$\alpha$ - und $\beta$-Relaxation verschieben sich in der Lage ihrer Relaxationszeit als Funktion der Temperatur unterschiedlich.

Eine Phasenverschiebung zwischen der Anregung und der Antwort des Systems bei dynamisch-mechanischer Analyse resultiert aus einer Resonanz von Spektroskopiefrequenz und $\alpha$ Relaxation. Diese kann aber mit anderen Relaxationen koppeln. Der Betrag des komplexwertigen Elastizitätsmoduls kann durch andere Relaxationen verändert werden.

Zumindest die $\alpha$-Relaxationszeit ist abhängig von der fiktiven Temperatur des Systems.

\section{Begründung}

Die erste Annahme ist motiviert durch die Vorgängerarbeit von [Rös04]. Darüber hinaus hat sich bereits in Unterkapitel 6.2 die Notwendigkeit der Existenz einer $\beta$-Relaxation als universelles Phänomen des Glasübergangs gezeigt.

Punkt Zwei kann als Voraussetzung akzeptiert werden, da nicht nur die Spektroskopiefrequenz die Messungen bestimmt, sondern aufgrund der Temperaturabhängigkeit der Relaxationszeiten der gegenwärtige Zustand des Nicht-Gleichgewichtssystems von der Heizrate abhängt. Für die Kreisfrequenzen $\omega$ der Spektroskopie $(S)$ mit Frequenz $f_{\mathrm{S}}$ und resultierend aus der Heizrate $R(H)$ können die folgenden Abschätzungen gemacht werden:

$$
\omega_{S}=2 \pi \cdot f_{S} \quad \omega_{H}=\frac{2 \pi}{t_{\text {char }}} \approx \frac{2 \pi}{1 \mathrm{~K} \cdot R^{-1}}=2 \pi \cdot R / \mathrm{K}
$$

Punkt Drei ist das Grundprinzip der mechanischen Spektroskopie und kann vorausgesetzt werden.

Punkt Vier ist als Annahme zu werten und setzt Punkt Eins voraus. Motiviert wird er durch die in Abbildung 6.2.1 gezeigte Temperaturabhängigkeit der Relaxationszeit von Orthoterphenyl. Die $\alpha$-Relaxationszeit kann dabei durch eine Vogel-Fulcher-Tamman-Funktion (Gleichung (8)) beschrieben werden, während die $\beta$-Relaxationszeit in guter Näherung ein Arrhenius-Verhalten (Gleichung (7)) zeigt.

Punkt Fünf kann mit folgender Begründung als gegeben angesehen werden: Bei Untersuchungen mittels DMA wird die Probe einer dynamischen Spannung ausgesetzt. Dies führt zu einer oszillierenden, phasenverschobenen Dehnung. Spannung und Dehnung sind über die Nachgiebigkeit $D^{*}$ verknüpft (Gleichung (15)). Diese ist gleich dem Kehrwert des Elastizitätsmoduls E* (Gleichung (17)). Um eine Zunahme des Phasenmoduls zu erreichen, muss eine Relaxation des Materials stattfinden, welche die eingetragene Dehnungsenergie in einer Zeit, die vergleichbar oder kürzer ist als das Inverse der Oszillationsfrequenz $f_{\mathrm{S}}$ (Punkt Zwei und Drei), konsumiert und in Wärme umwandelt. Ein solcher nicht-reversibler Anteil der Dehnung bedingt die Aktivität der $\alpha$-Relaxation. Diese kann jedoch noch weit von ihrem Maximum entfernt sein, wenn eine weitere Relaxation mit der $\alpha$-Relaxation koppelt. Ist die $\alpha$ Relaxation noch nicht bei der Spektroskopiefrequenz aktiv, so kann eine Relaxation, die somit nicht in einer Änderung des Phasenwinkels sichtbar wird, sich dennoch im Speicher- und Verlustmodul niederschlagen. Dies folgt aus den Gleichungen $(13,14,16)$ : 


$$
E^{\prime}=\left|E^{*}\right| \cdot \cos (\delta) \quad E^{\prime \prime}=\left|E^{*}\right| \cdot \sin (\delta) \quad\left|E^{*}\right|=\frac{\sigma_{0}}{\varepsilon_{0}}
$$

Dabei ist $\sigma_{0}$ die Amplitude der oszillierenden Spannung und $\varepsilon_{0}$ die Dehnungsamplitude. Bei gleich bleibender dynamischer Kraft ändert sich die Spannungsamplitude nicht ohne irreversible Verformung, während sich die Antwort des Systems $\varepsilon_{0}$ ändern kann, wenn das Material seine Steifigkeit ändert. Dieses Phänomen ist unter anderem beim Vergleich von Abbildung 4.6.5 mit Abbildung 6.5.1 zu beobachten. Die Änderung des Speichermoduls beim ersten Aufheizen setzt bei $460 \mathrm{~K}$ ein, der $\tan \delta$ beginnt sich aber erst bei $493 \mathrm{~K}$ zu ändern.

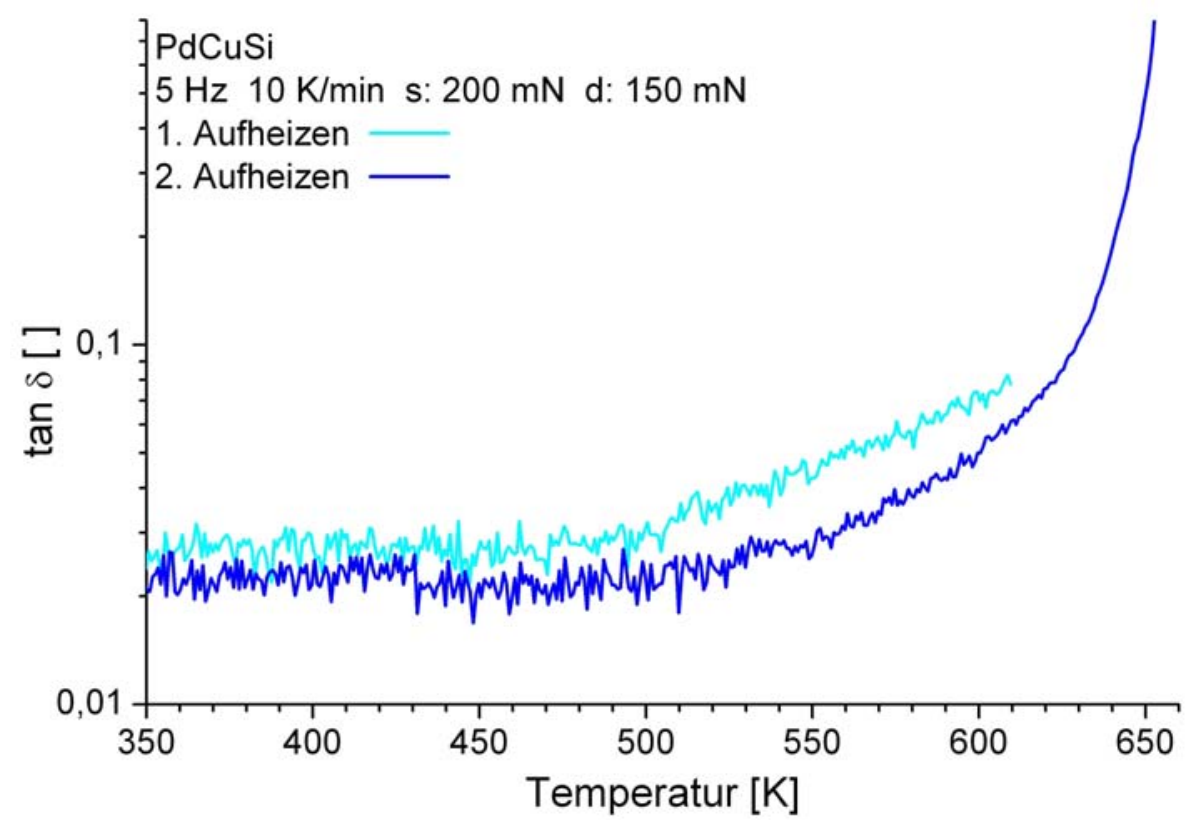

Abb. 6.5.1: Der Verlauf des tan $\delta$ zeigt erst Relaxationseffekte, wenn die Probe deformiert wird (vgl. Abb. 4.6.5).

Punkt Sechs beruht auf der Tatsache, dass mit verschiedenen Kühlraten aus der Schmelze verschiedene Gläser entstehen. Das Auslagern einer Probe im Bereich der $\alpha$-Relaxation und anschließendes Abkühlen mit einer im Vergleich zum ersten Abschrecken langsameren Rate führt zu einem Glas mit einer niedrigeren fiktiven Temperatur (vgl. Kap. 2.3). Mit anderen Worten verschiebt sich beim Altern in der Nähe des Zustands der unterkühlten Schmelze die $\alpha$-Relaxation hin zu tieferen Temperaturen. Da die $\alpha$-Relaxation aus einer Verteilung von Relaxationszeiten besteht und das makroskopische Fließen der Probe die DMA-Messung beendet, kann eine Alterung, bei der sich das gesamte Spektrum der Relaxationszeitverteilung des $\alpha$-Prozess resonant zu $\omega_{\mathrm{H}}$ verhält, nicht erreicht werden.

\section{Gegensätzlichkeit zwischen Alterungsprozess und $\alpha$-Relaxation}

Bereits in Unterkapitel 6.2 konnte gezeigt werden, dass die Anpassung einer Fitfunktion unter der Annahme der Existenz einer einzigen $\alpha$-Relaxation nicht zufrieden stellend gelingt. Daraus wurde das Vorhandensein einer sekundären Relaxation geschlossen. Es gibt aber noch einen weiteren Prozess, der sich nicht mit der $\alpha$-Relaxation erklären lässt: Das Altern der Ausgangsproben beim ersten Aufheizen weit unterhalb des Glasübergangs. 
Dieser Alterungseffekt zeigt sich sowohl bei $\mathrm{ZrAlCu}$ in Abbildung 4.6.2 als auch bei PdCuSi in Abbildung 4.6.5 in Form eines Anstiegs des Speichermoduls. Würde der $\alpha$-Relaxation dieser Prozess zugeschrieben, so würde dies bedeuten, dass zwischen dieser Resonanz (2. und 3.) aus $\alpha$ und $\omega_{\mathrm{H}}$ und jener zwischen $\alpha$ und $\omega_{\mathrm{S}}$ am dynamischen Glasübergang für PdCuSi mehr als $150 \mathrm{~K}$ liegen. Eine derart große Verschiebung müsste sich auch durch eine Variation der Spektroskopiefrequenz erreichen lassen. Bei der Frequenzänderung von $10 \mathrm{~Hz}$ auf $0,1 \mathrm{~Hz}$ in Abbildung 4.4.6 wird eine resultierende Temperaturdifferenz der $\tan \delta$-Onsets in Tabelle 4.4.4 von lediglich $12 \mathrm{~K}$ erreicht. Somit lässt sich eine Verschiebung von $150 \mathrm{~K}$, resultierend aus dem Unterschied zwischen Heizrate von $0,17 \mathrm{~K} / \mathrm{s}$ und der Spektroskopiefrequenz von $5 \mathrm{~Hz}$, nicht rechtfertigen.

Derselbe Widerspruch ergibt sich beim Betrachten der Verschiebung, welche durch Variation der Heizrate erreicht wird: Aus heizratenabhängigen DSC-Messungen an $\mathrm{ZrAlCu}$ ist eine Verschiebung des Glasübergangs von weniger als $10 \mathrm{~K} \mathrm{zu}$ erwarten, wenn die Heizrate von $20 \mathrm{~K} / \mathrm{min}$ auf $60 \mathrm{~K} / \mathrm{min}$ erhöht wird. Zwischen Alterung und dynamischem Glasübergang bei $\mathrm{ZrAlCu}$ bei $20 \mathrm{~K} / \mathrm{min}$ und einer Frequenz von $f_{\mathrm{S}}=1 \mathrm{~Hz}$, welche etwa mit einer Heizrate von $60 \mathrm{~K} / \mathrm{min}$ zu vergleichen ist (Gleichung (29)), liegen dagegen mehr als $170 \mathrm{~K}$ (Abb. 4.6.2).

Auch die DSC-Messungen in Abbildungen 4.3.3+5 scheinen widersprüchlich zu sein, wenn der Alterungsprozess der $\alpha$-Relaxation zugeschrieben wird: Allgemein sollte bei DSCMessungen stets nur ein Onset zu beobachten sein, wenn es nur eine $\alpha$-Relaxation gibt, da die DSC nur über eine charakteristische Zeitskala verfügt, welche invers proportional zur Heizrate ist. Eine Alterung in der Nähe dieses Übergangs würde zu einer Verschiebung zu tieferen Temperaturen führen (6.). Diese Erwartung wird nicht erfüllt. Bei Auslagerungsexperimenten an $\mathrm{PdCuSi}$, bei denen für eine vorgegebene Zeit die Temperatur konstant gehalten wird und zwar deutlich unterhalb des Glasübergangs beim Heizen mit $5 \mathrm{~K} / \mathrm{min}$, ist genau das Gegenteil zu beobachten. Beim auf den Auslagerungsschritt folgenden Aufheizen verschieben sich sowohl Onset als auch Wendepunkt des Glasübergangs geringfügig zu höheren Temperaturen. Dagegen zeigt sich die Tieftemperaturflanke deutlich verändert. Der Anstieg erfolgt abrupter, ebenso wie der Abfall. Es ist schwer, eine genaue Aussage über die Fläche unter der Kurve und somit über die Menge der aufgenommenen Energie bei Ausgangsproben und gealterter Probe zu machen (vgl. [KAH06]).

Dass es sich beim Altern nicht um die $\alpha$-Relaxation handeln kann, zeigt sich aber auch bereits während der Auslagerungsphase: Beim Messen von PdCuSi-Augangsproben in der DSC bei konstanter Temperatur von $573 \mathrm{~K}$ (vgl. Abb. 4.3.4), wird eine Relaxation im Wärmefluss beobachtet. Diese fehlt bei Proben, welche zuvor bei $616 \mathrm{~K}$ ausgelagert wurden. Würde es sich dabei um die $\alpha$-Relaxation oder ein langsames Einpendeln der Temperatur handeln, sollten auch die ausgelagerten Proben diesen Effekt zeigen. Darüber hinaus ist bei einer $\alpha$-Relaxation ein endothermes Maximum zu erwarten, die abklingende Störung ist dagegen exotherm.

\section{Folgerung}

Da das Phänomen der Alterung nicht mit dem Auftreten der $\alpha$-Relaxation erklärt werden kann ist es sinnvoll, den Alterungsprozess mit der $\beta$-Relaxation zu verknüpfen. 
Der mit der Alterung verbundene Anstieg im Speichermodul wird als Resonanz zwischen der Heizrate und der Relaxationszeit der $\beta$-Relaxation interpretiert.

Diese Folgerung erlaubt die Deutung sämtlicher beobachteten Relaxationsphänomene als Resonanzbedingungen zwischen den zwei charakteristischen Beobachtungszeiten, die sich in Abfragefrequenzen übersetzen lassen und den zwei temperaturabhängigen Relaxationszeiten des $\alpha$ - und $\beta$-Prozesses. Die Temperaturabhängigkeit der Relaxationszeiten (vgl. Abb.6.2.1) ist dabei nicht statisch sondern verschiebt sich, wenn eine oder mehrere Relaxationen aktiv sind. Es sind keine weiteren, tertiären Relaxationen zur Deutung nötig. Die Resonanzen treten wie folgt auf, diskutiert am Beispiel von PdCuSi in Abbildung 6.5.2:

$\omega_{\mathrm{H}} \approx \tau_{\beta}{ }^{-1}:$ Im System eingefrorene Verspannungen können durch einen $\beta$-Prozess relaxieren. $\left|E^{*}\right|$ ändert sich (Gleichung (39)), was sich in Form eines Anstiegs im Speichermodul zeigt. Die Probe kann nicht makroskopisch deformiert werden, deshalb bleibt der Phasenwinkel im Rahmen der Messgenauigkeit zunächst unverändert (vgl. Abb. 6.5 .2 bei $0,5 \mathrm{~K} / \mathrm{min}$ ). Die $\beta$-Relaxation ist nun auf diesen Zeitskalen aktiv und kann sich verschieben (Alterung), Abbildung 6.2.1 ist nicht länger statisch. Wieweit sich $\tau_{\beta}$ zu tieferen Temperaturen bzw. kürzeren Relaxationszeiten verschiebt, ist unklar.

$\omega_{\mathrm{S}}=\tau_{\beta}{ }^{-1}:$ Ist durch die $\beta$-Relaxation in Abwesenheit einer $\alpha$-Relaxation eine irreversible Deformation der Probe möglich, sollte nun einen Anstieg im $\tan \delta \mathrm{zu}$ beobachten sein. Ist durch die $\beta$-Relaxation keine irreversible Deformation möglich, so erfolgt eine Ankopplung der äußeren Anregung an die $\beta$-Relaxation über die $\alpha$-Relaxation. Eine Unterscheidung zwischen den beiden Szenarien ist im Rahmen der Genauigkeit der DMA nicht möglich. Fest steht, dass der Anstieg des $\tan \delta$ zwei Onset-Punkte besitzt (Onset zu Punkt Zwei und Onset zu Punkt Vier).

$\omega_{\mathrm{H}} \approx \tau_{\alpha}{ }^{-1}$ : Die $\alpha$-Relaxation ist nun auf Zeiten der Heizrate aktiv und kann $\left|E^{*}\right|$ verändern (Verschiebung der fiktiven Temperatur, vgl. Kap. 2.3). Eine Verschiebung von beiden Relaxationszeiten ist nun möglich [KAH06]. Dies bewirkt, dass Punkt Vier bald eintritt (vgl. Kap. 2.3). Der Onset zu dieser Resonanz zeigt sich in Form des Einsetzens des viskosen Fließens bei 646(10) K bzw. 690(20) K bei einer Heizrate von 0,5 K bzw. $20 \mathrm{~K}$ (Abb. 6.5.2).

$\omega_{\mathrm{S}}=\tau_{\alpha}^{-1}$ : Die $\alpha$-Relaxation ist resonant zur Spektroskopiefrequenz. Dieser Zustand wird schon nicht mehr erreicht, da dies einer Resonanzkatastrophe gleichkäme. Das Auseinanderfließen der Probe (Punkt Drei) oder die Kristallisation kommen dieser zuvor. Nur der Onset zu dieser Relaxation ist messbar. Dieser kann im Bereich der Punkte Zwei und Drei liegen. Ist die Spektroskopiefrequenz nicht zu verschieden von $\tau_{\beta}{ }^{-1}$, so ist ein Koppeln der einsetzenden $\alpha$-Relaxation mit der $\beta$-Relaxation in Form eines ,excess wing“ möglich. 


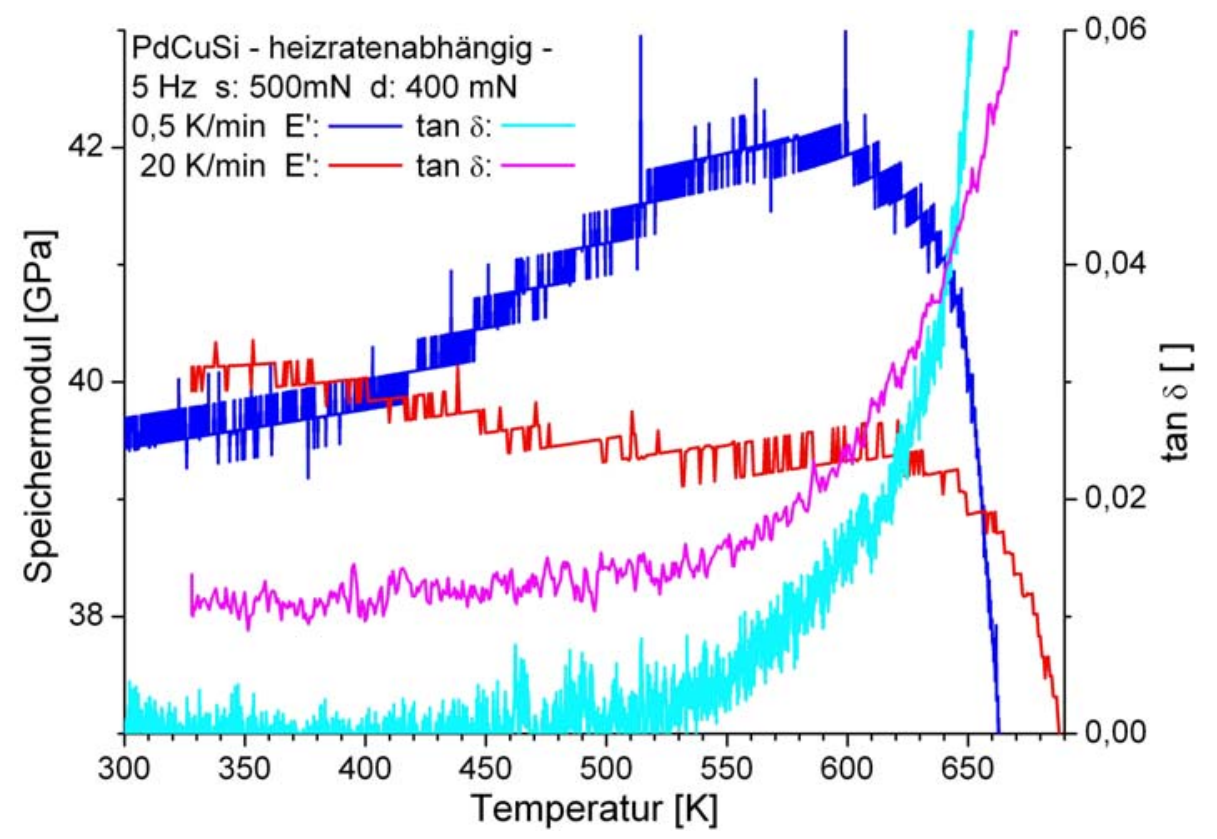

Abb. 6.5.2: Anstieg im Speichermodul von PdCuSi bei Heizraten von 0,5 K/min und $20 \mathrm{~K} / \mathrm{min}$ und Verlauf der zugehörigen $\tan \delta$, identisch zu Abb. 4.6.6

Auf diese Weise können alle im zweiten Teil von Kapitel 4.4 gezeigten Parameterabhängigkeiten der Messungsverläufe erklärt werden. Abbildung 4.6.5 zeigt einen Alterungsanstieg, der in Abbildung 4.6.1 aufgrund der anfänglichen Heizrate von $20 \mathrm{~K} / \mathrm{min}$ nicht zu erkennen ist (Punkt Eins bis Punkt Drei fallen zusammen). Bei $f_{\mathrm{S}}=5 \mathrm{~Hz}$ ist eine deutlicher ausgeprägte Tieftemperaturflanke zu sehen, weil $f_{\mathrm{S}}$ näher an $\tau_{\beta}{ }^{-1}$ ist. Beim zweiten Aufheizen entfällt Punkt Eins, weil die Verspannung schon im ersten Durchlauf abgeklungen ist. Der NCL ist im Verlust besser zu erkennen, weil sich $\left|E^{*}\right|$ kaum ändert. Gleiches gilt für die Cole-Coleangepasste Messung in Abbildung 6.2.4.

Auch die in vorangegangenen Arbeiten durchgeführten spektroskopischen Untersuchungen an metallischen Gläsern können anhand des obigen Resonanzschemas eingeordnet werden:

So zeigte [KAH04], dass sich der Speicheranteil des Schermoduls durch Auslagern weit unter der $\alpha$-Relaxation verändern kann $\left(t_{\text {Auslagerung }} \approx \tau_{\text {b }}\right.$ ). Der angestrebte Gleichgewichtswert erweist sich dabei als temperaturabhängig. Ob in Abbildung 6.5.1 eine $\beta$-Relaxation sichtbar ist, weil beim ersten Aufheizen die Zeit zu kurz war um die anfängliche Spannung zu relaxieren, oder ob das Material sogar eine Verjüngung zeigt, konnte nicht abschließend geklärt werden. Das frühe Absinken des Speichermoduls kann auch schon zu der zur Heizrate resonant werdenden $\alpha$-Relaxation gehören.

Bei den DPO-Experimenten an $\mathrm{ZrAlCu}$ in [RÖS04] war die Spektroskopiefrequenz $f_{\mathrm{S}}=5,44 \mathrm{kHz}$ viel zu hoch, um eine $\alpha$-Relaxation vor dem Eintreten der Kristallisation dynamisch zu messen. Es werden nur Resonanz eins und zwei beobachtet. Erstere führt auch bei Paddeln mit einer Tantal-Diffusionsbarriere zu deutlichen Verschiebungen der Eigenfrequenz bei Temperaturrampen bis $594 \mathrm{~K}$, ohne dass sich der tan $\delta$ ändert (vgl. [RÖS04] Abb. $5.21 \&$ 5.23 darin). Der Grund dafür ist, dass sehr kleine Heizraten im Bereich von $0,2 \mathrm{~K} / \mathrm{min}$ verwendet worden sind. Der Onset zur der an die Frequenz koppelnden $\beta$-Relaxation ist bei etwa $625 \mathrm{~K}$ in Abbildung 6.2.2 zu sehen. Die Änderung des tan $\delta$ ist dabei weniger als $3 \cdot 10^{-6}$. Es ist nicht klar, ob diese Differenz daraus resultiert, dass es zu einem Koppeln mit der noch weit 
entfernten $\alpha$-Relaxation kommt, oder ob auch der $\beta$-Prozess zu einer kleinen, irreversiblen Deformation der Probe führen kann. Dies wirft direkt die Frage auf, welcher Art die zur $\beta$ Relaxation führenden Prozesse sind, welche im übernächsten Abschnitt diskutiert wird.

\section{Zeit-Temperatur-Verschiebung der $\beta$-Relaxation}

Da es sich also beim ersten Anstieg des Speichermoduls auf der Zeitskala der Heizrate um dieselbe Relaxation handelt, wie die (langsame) $\beta$-Relaxation auf der Zeitskala der Schwingungsdauer der mechanischen Spektroskopie, muss sich dies in der Temperaturabhängigkeit der Relaxationszeit widerspiegeln. Dieser Effekt sollte sich sowohl bei Variation der Spektroskopiefrequenz als auch der Heizrate zeigen. Da nicht klar ist, ob die $\beta$-Relaxation nur spektroskopisch messbar ist, wenn eine Ankopplung an die $\alpha$-Relaxation möglich ist, bietet sich eine Variation der Frequenz nicht an. Zudem zeigt auch die $\alpha$-Relaxation eine Frequenzabhängigkeit (vgl. Abb. 4.4.6). Deutlich praktikabler ist die heizratenabhängige Messung bei konstanter Frequenz, wie in den Abbildungen 4.6.6f für $\mathrm{PdCuSi}$ und $\mathrm{ZrAlCu}$ gezeigt. Eine geeignete Messgröße ist dabei der Onset zum Anstieg des Speichermoduls. Dieser tritt auf, wenn die temperaturabhängige $\beta$-Relaxationszeit in den Bereich der Zeit der Beobachtung absinkt (Onset zu Punkt Eins auf der vorangegangenen Seite). Ebenfalls gut zu bestimmen ist der Wendepunkt des Anstiegs. Dabei handelt es sich nicht zwangsläufig um die Temperatur des Eintretens der Resonanzbedingung $\omega_{\mathrm{H}} \approx \tau_{\beta}{ }^{-1}$. Ist die Verspannung des Materials abgeklungen bevor die Resonanz erreicht ist, liegt der Wendepunkt bei tieferen Temperaturen als die Resonanz. Die beiden sollten aber nicht weit auseinander liegen aufgrund der Tatsache, dass es sich bei der $\beta$-Relaxationszeit wahrscheinlich, wie auch bei der $\alpha$-Relaxation, nur um den Mittelwert einer Verteilung von Relaxationszeiten handelt. Um eine vollständige Entspannung des Materials zu erreichen müssen alle Einzelrelaxationen ablaufen können. Die Lage des Wendepunktes kann auch beeinflusst werden, wenn es zu einer Überlagerung zwischen $\beta$ - und $\alpha$-Relaxation kommt. Dies ist in den Abbildungen 4.6.6f bei hohen Heizraten zu beobachten. Da die Resonanz $\omega_{\mathrm{H}} \approx \tau_{\alpha}{ }^{-1}$ nicht beobachtet werden kann, weil das Material zuvor auseinander fließt oder kristallisiert, bleibt der Onset dieses viskosen Fließens als zusätzliche Messgröße. Sie dient zur besseren Einschätzbarkeit der Frage, wie die Relaxationszeiten bei hohen Temperaturen zusammen laufen.

Die folgenden Abbildungen 6.5.3f sind in Anlehnung an die Darstellung des temperaturabhängigen Verlaufs der Relaxationszeiten von Orthoterphenyl (Abb. 6.2.1) aufgetragen. Der einfachste Fall dieser Abhängigkeit ist ein Arrhenius-Gesetz (nach Kap. 2.4, Gleichung (7)):

$$
\begin{gathered}
\tau(T)=\tau_{0} \exp \left(\frac{E_{A}}{k_{B} T}\right) \\
\Leftrightarrow \log _{10}(\tau)=\underbrace{E_{A} \frac{\log _{10}(e)}{1000 \mathrm{~K} \cdot k_{B}}}_{m} \frac{1000 \mathrm{~K}}{T}+\log _{10}\left(\tau_{0}\right)
\end{gathered}
$$

Der Verlauf der logarithmierten Relaxationszeit als Funktion von $1000 \mathrm{~K} / \mathrm{T}$ sollte demnach linear sein, wobei die Aktivierungsenergie $E_{\mathrm{A}}$ proportional zur Steigung $m$ ist. Erfüllt $\omega_{\mathrm{H}}$ die Resonanzbedingung (Kap. 2.4, Gleichung (18)), so ist nach Gleichung (29) die Auftragung der logarithmierten, inversen Heizrate $R$ anstelle der logarithmierten Relaxationszeit sinnvoll. Durch das Multiplizieren der Rate $R$ mit dem Wert $1 \mathrm{~K}$ wird eine Größe erreicht, welche zu 
einer charakteristischen Zeit des Aufheizens proportional ist. Im Fall eines Arrhenius-Gesetz müsste sich folglich ein linearer Verlauf ergeben, da gilt:

$$
\begin{aligned}
& \tau=\frac{1}{\omega_{H}} \propto \frac{1 \mathrm{~K}}{R} \\
\Rightarrow & \log _{10}\left(\frac{1 \mathrm{~K}}{R}\right)=\underbrace{E_{A} \frac{\log _{10}(e)}{1000 \mathrm{~K} \cdot k_{B}}}_{m} \frac{1000 \mathrm{~K}}{T}+\text { const }
\end{aligned}
$$

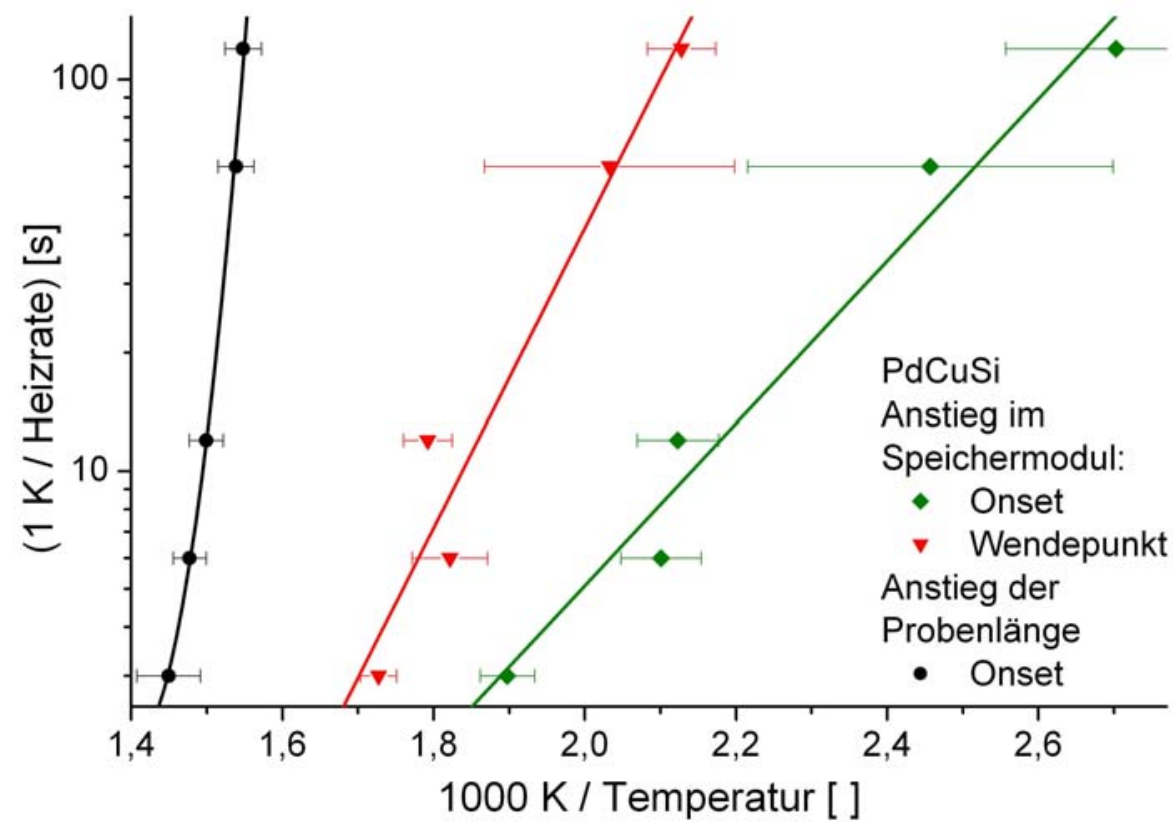

Abb. 6.5.3: Heizratenabhängigkeit der Temperaturen des Auftretens von $\alpha$ - und $\beta$-Relaxation bei PdCuSi

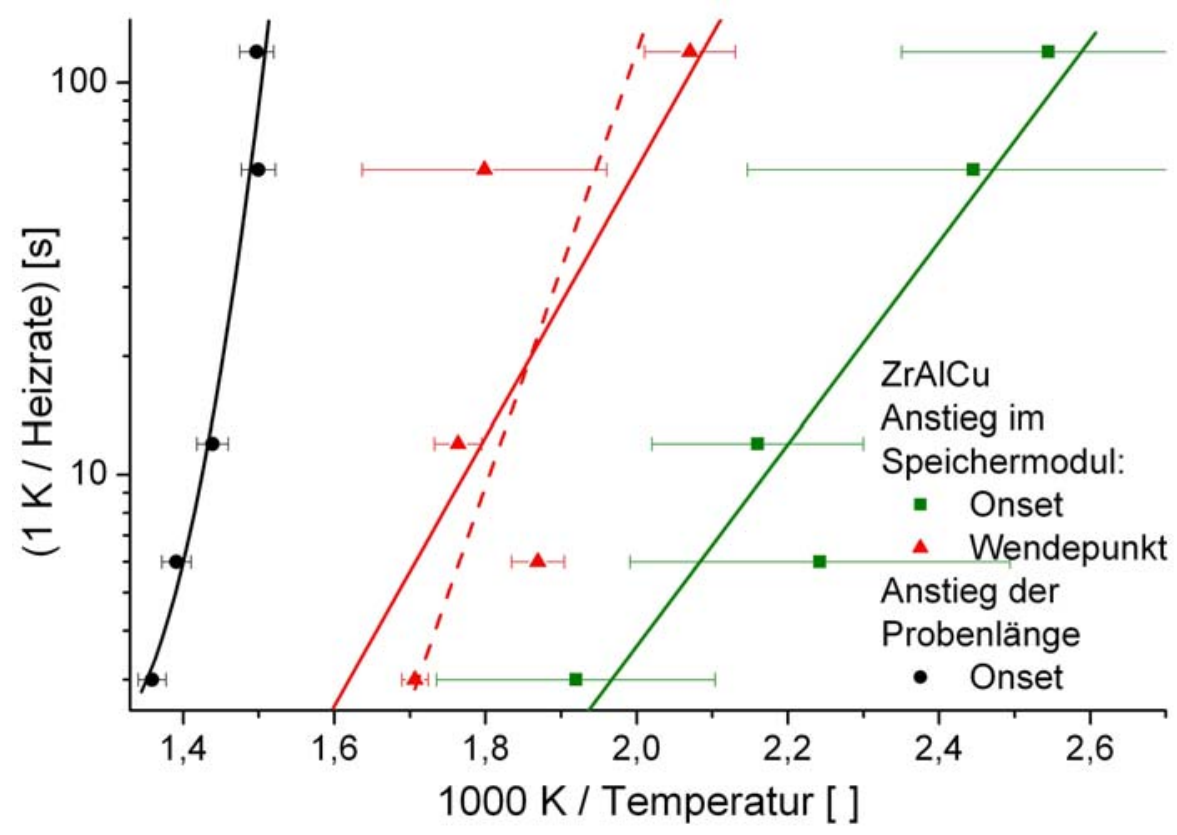

Abb. 6.5.4: Heizratenabhängigkeit der Temperaturen des Auftretens von $\alpha$ - und $\beta$-Relaxation bei $\mathrm{ZrAlCu}$ 
Sowohl bei PdCuSi als auch bei ZrAlCu fällt auf, dass die Verläufe aller drei Messgrößen zusammenlaufen (Onset der $\alpha$-Relaxation, Onset der $\beta$-Relaxation und Wendepunkt des Anstiegs). Durch die Onset- und Wendepunkte der Anstiege in den Speichermoduln sind Ausgleichsgerade gezeichnet worden (durchgezogene Linien). Bei $\mathrm{ZrAlCu}$ ist aber auch ein deutlich steilerer Kurvenverlauf möglich, wie mit der manuell bestimmten, gestrichelten Linie angedeutet. Da die Temperaturabhängigkeit der $\alpha$-Relaxation einem Vogel-FulcherTammann-Verhalten folgt (vgl. Kap. 2.4 und Kap. 6.2), ist in die Temperaturabhängigkeit der Onsets der $\alpha$-Relaxation eine gekrümmte Trendlinie eingezeichnet. Da die Resonanz in einem Nicht-Gleichgewichts-Zustand vorliegt, ist es nicht sinnvoll, den Verlauf mit einem VogelFulcher-Tammann-Gesetz anzupassen. Die Fitparameter besäßen wenig Aussagekraft.

Somit ist auch eine Bestimmung von Schnittpunkten schwierig. Dass es sich um ein und dieselbe Relaxation handeln kann, ist besser aus den Auftragungen von Speichermodul und $\tan \delta$ in Abbildung 6.5.2 für PdCuSi und Abbildung 4.6.7 für $\mathrm{ZrAlCu}$ ersichtlich. Bei letzterer überlappen sich sogar die Fehlerbereiche der Werte für den Onset des Anstiegs im Speichermodul mit dem des ersten Abknickens des tan $\delta$. Eine Frequenz von $5 \mathrm{~Hz}$ ist also dem Heizen mit $1 / 3 \mathrm{~K} / \mathrm{s}$ schon sehr nahe.

Ob die Verläufe von Onset und Wendepunkt der $\beta$-Relaxation in den Abbildungen 6.5.3f einem Arrhenius- oder einem Vogel-Fulcher-Tammann-Gesetz folgen, ist im Rahmen der Genauigkeit und aufgrund des zu kleinen Bereichs an Heizraten nicht festzustellen. Es fällt aber auf, dass die Steigungen deutlich kleiner sind als die der $\alpha$-Relaxation. Dies spiegelt ebenfalls die großen Temperaturverschiebungen im Auftreten der $\beta$-Relaxation beim ändern der Zeitskala wider. Die aus den Steigungen der Wendepunkte gewonnenen Aktivierungsenergien betragen 0,66(10) eV für PdCuSi und 0,59(34) eV für ZrAlCu bzw. 0,96(50) eV für die gestrichelte Linie.

Diese Werte sind allerdings mit Vorsicht zu genießen und stellen eher eine obere Grenze dar: Es ist einerseits nicht klar, ob sich die $\beta$-Relaxation durch Alterung zu kleineren Werten verschieben lässt und inwieweit diese Kühlratenabhängig (während der Herstellung) ist. Andererseits sinkt die Kühlrate beim Raschabschrecken mit fallender Temperatur. Somit kann es bereits beim Abkühlen zu einer teilweisen Entspannung gekommen sein, sofern es sich auch hier um eine Verteilung von Relaxationszeiten handelt. Dies würde sowohl den Onset als auch den Wendepunkt zu höheren Temperaturen verschieben, was insbesondere die bei kleinen Heizraten gewonnen Werte beeinflussen würde.

Folglich wären Messungen an Gläsern wünschenswert, die bei hohen Kühlraten hergestellten sind. Liegt dabei die Endtemperatur des Abschreckvorgangs weit unterhalb der Temperatur, bei der bereits in der Zeit vor der Messung Relaxationen ablaufen können, so wäre der Verlauf deutlich besser zu bestimmen. Eine Kühlung am Substrathalter des DPO könnte sich dazu als sehr nützlich erweisen.

\section{Ursachen für eine $\beta$-Relaxation}

Das Wesen der $\beta$-Relaxation ist nach wie vor unverstanden. Klar ist, dass es sich nicht um eine Umlagerung von einzelnen oder wenigen Atomen handeln kann, bei der sich Nachbarschaftsverhältnisse der Atome untereinander ändern. Dies erfordert eine $\alpha$-Relaxation, wie kürzlich in [JS05] theoretisch beschrieben. Dabei wird eine sinusförmige Energielandschaft 
angenommen, deren Krümmung sich im Schermudul und somit auch in $\left|E^{*}\right|$ widerspiegelt. Es wird gefolgert, dass es eine untere Grenze von etwa 150 Atomen gibt, die in einer ScherTransformations-Zone kooperativ aneinander abgleiten, umgeben von einer noch größeren Zahl von Atomen, die eine elastische Deformation vollziehen. Dieses Bild könnte durch eine zusätzliche Feinstruktur, wie in [DS01] beschrieben, ergänzt werden.

Kürzliche Simulationsergebnisse legen nahe, dass es sich auch bei der $\beta$-Relaxation um einen kooperativen Prozess von mehreren Atomen handelt. Dieser soll jedoch einen reversiblen Charakter besitzen, solange es nicht zu einer Überlagerung von mehreren kooperativ relaxierenden Bereichen kommt [Tei05, VLZ05].

Eine theoretische Beschreibung, die beide Relaxationen berücksichtig und das Verhalten in der Nähe des Glasübergangs über den gesamten Bereich der vorkommenden Relaxationszeiten beschreiben kann, fehlt bisher noch.

\subsection{Sekundäre Relaxationen im Copolymer: Eine Arbeitshypothese}

Das Verständnis der in einem Material auftretenden Relaxationen spielt nicht nur bei Metallen, sondern bei allen Werkstoffen eine zentrale Rolle in deren Anwendung. Ziel dieser Arbeit ist es, die makroskopischen, mechanischen Eigenschaften und das Relaxationsspektrum von Copolymeren zu untersuchen, deren mikroskopische chemische Struktur während der Synthese gezielt manipuliert wurde. Dabei zeigt sich, dass die komplexere Struktur der Polymere mit einer größeren Anzahl von Relaxationsprozessen einhergeht, wie in Kapitel 2.6 beschrieben. So geht der Glasübergang in Polymeren, der in der Chemie auch als Erweichungsübergang bezeichnet wird, in zwei Hauptprozessen vonstatten: Zunächst kommt es beim Erhöhen der Temperatur zu einer Erweichung durch eine Zunahme in der Mobilität der Segmentbewegung [MRW67]. Dadurch wird eine Deformation des Polymers möglich, weswegen dieser Prozess in dieser Arbeit als $\alpha$-Relaxation bezeichnet wird. Bei noch höheren Temperaturen ist ein Abgleiten der Ketten auf ganzer Länge möglich, welches zu einem viskosen Fließen führt. Dies wird auch als ,normal mode“ [MRW67] beschrieben und daher in dieser Arbeit als $\alpha$ 'Relaxation bezeichnet. Bedingt durch die komplizierte Geometrie der Polymere geschieht der Übergang vom Glas in die Flüssigkeit in zwei Stufen. Damit ist klar, dass eine Änderung dieser Geometrie zu einer Änderung der Relaxationsprozesse führt. Bei sehr langen Ketten können $\alpha$ - und $\alpha^{\prime}$-Relaxation deutlich voneinander getrennt sein. Dazwischen liegt ein Bereich, in dem der Kunststoff gummielastisch auf Deformation reagiert. Wird durch Vernetzung der Polymere eine $\alpha$ '-Relaxation gänzlich unterdrückt, so kann diese elastische Deformation auch über lange Zeiten gespeichert werden. Diese Stoffklasse wird umgangssprachlich als Gummi bezeichnet. Bei einer Reduktion der Kettenlänge in den Bereich der Segmentbewegung können $\alpha$ - und $\alpha$-Relaxation nicht mehr voneinander getrennt werden. Eine darüber hinaus gehende Verkürzung der Kettenlänge führt dann zu einer Verschiebung der $\alpha$-Relaxation [SPE92]. Eine weitere Beeinflussung der Geometrie stellt der Übergang zu dünnen Filmen dar, welcher ebenfalls die Beweglichkeit der Polymere beeinflusst. Dieser Effekt wird als „spatial“" [SK03] oder , geometric confinement“ [SGS02] bezeichnet. Beim dabei entstehenden Kontakt mit festen Wänden kann es zu festen Kontaktpunkten mit dem Polymer kommen. Die bei erhöhter Temperatur frei beweglichen „dangling ends“ (baumelnden Enden) führen zu einem zusätzlichen Relaxationsmaximum im Spektrum von dielektrischen Messungen, das zwischen $\alpha$ - und $\alpha$ '-Relaxation liegt [SK03]. Ob auch bei freien Oberflächen eine Verände- 
rung der Relaxationen durch eine Erhöhung der Beweglichkeit zu beobachten ist, wird zurzeit kontrovers diskutiert und untersucht [HAR06].

In dieser Arbeit liegt das Hauptinteresse auf solchen Relaxationen, die auf noch kleineren Längenskalen bei Temperaturen unterhalb der $\alpha$-Relaxation, respektive höheren Frequenzen, liegen. Die Existenz einer im Vorherigen als universell postulierten langsamen $\beta$-Relaxation wird dabei auch hier angenommen. Sie kann bei Polymeren allerdings nicht zweifelsfrei nachgewiesen werden. Dies ist begründet durch die Tatsache, dass eine komplexere Struktur der Moleküle zu einer Vielzahl von Relaxationen führen kann. Es gibt aber auch Studien, die behaupten, eine langsame $\beta$-Relaxation bei organischen Molekülen zu beobachten. Darin wird angenommen, dass diese nicht zu weiteren strukturellen Relaxationen fähig sind, z.B. [LWU05].

Die Besonderheit der synthetisierten Copolymere, die in dieser Arbeit untersucht werden, ist der große Unterschied des chemischen Charakters der eingebauten Monomere. Wie in Kapitel 3.2 beschrieben, zeichnen sich die Ethylen-Einheiten in Poly(Ethylen-co-Methacrylsäure) (EMAA) durch eine einfache Struktur und geringe Polarität aus. Die anziehende Wechselwirkung mit benachbarten Gruppen beschränkt sich auf Van-der-Waals-Kräfte. Die Methacrylsäure (MAA)-Gruppen dagegen verfügen über zwei Seitengruppen, von denen die Carboxylfunktion polar ist und über Wasserstoffbrückenbindungen inter- oder intramolekulare Bindungen mit benachbarten Carboxylfunktionen eingehen kann. Sie können dadurch weitaus stärkere Bindungen eingehen als benachbarte Ethylen-Einheiten. Diese gegensätzlichen Eigenschaften führen dazu, dass eine Mischung der Monomere nur im superkritischen Zustand des Ethens bei hohen Drücken und hohen Temperaturen möglich ist. Wären die unterschiedlichen Polymereinheiten unter Normalbedingungen nicht fest miteinander in der Copolymerstruktur verbunden, so wären sie nicht mischbar.

Diese divergierenden Eigenschaften von aneinander gereihten Einheiten entlang des Makromoleküls führen zu der folgenden Arbeitshypothese: Befinden sich im Copolymer, durch Synthese bedingt, ganze Abschnitte einer Monomersorte und ist die Länge dieser Blöcke vergleichbar mit der Längenskala einer oder mehrerer der vorherrschenden Relaxationen, so sollte sich diese charakteristische Längenskala im Relaxationspektrum durch zusätzliche Maxima im Verlustmodul widerspiegeln und auch im DSC-Signal sichtbar sein.

Dabei wird sich zu Nutze gemacht, dass die Verteilung der Monomereinheiten entlang der Kette durch die Wahl des Synthesedrucks manipuliert werden kann. Bei hohem Druck ist die Tendenz der MAA-Monomere zu einer spezifischen Bindung an radikalischen Einheiten gleicher Sorte geringer, da sich das System in einem Zustand höherer Energie befindet. Dies resultiert in einer statistischeren Verteilung der Monomereinheiten im EMAA (Abb. 6.6.1 a)) als bei niedrigerem Druck. Die Reduktion des Drucks auf Werte knapp oberhalb der Entmischungsgrenze (,cloud point") führt zu einer weniger zufälligen Verteilung; es entstehen kurze Blöcke von Monomeren einer Sorte (Abb. 6.6.1 b)). Es wird vermutet, dass sich das Vorhandensein einer solchen charakteristischen Länge auf die Relaxationen auswirkt, die auf dieser Skala aktiv sind. Weniger statistische Copolymere, die bei geringerem Druck hergestellt wurden, sollten somit zusätzliche Relaxationsmaxima im Verlustspektrum zeigen, die bei Copolymeren mit statistischer Monomerverteilung (bei hohem Synthesedruck) fehlen, wie in Abbildung 6.6.1 d) und c) gezeigt. Eine Erhöhung des sehr niedrig gewählten MAA-Anteils 
sollte dann zu einer Verkürzung der zwischen den MAA-Blöcken liegenden Ethylenblöcke führen und sich in einer Verschiebung der Relaxationsmaxima manifestieren.

a)

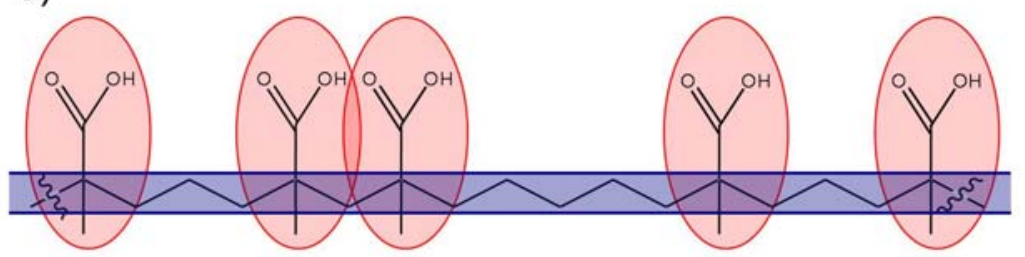

b)

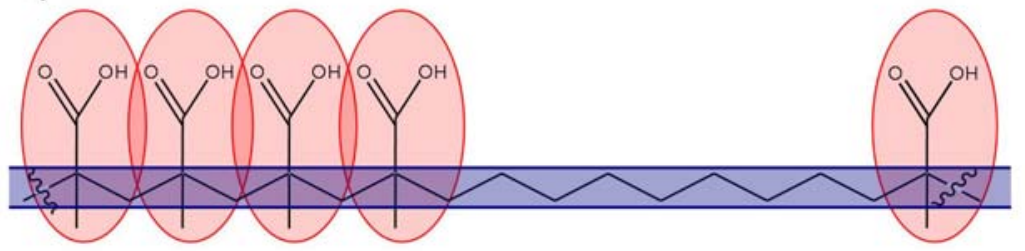

c)

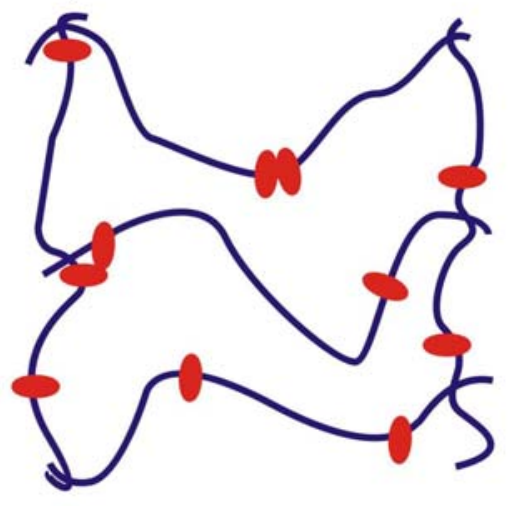

d)

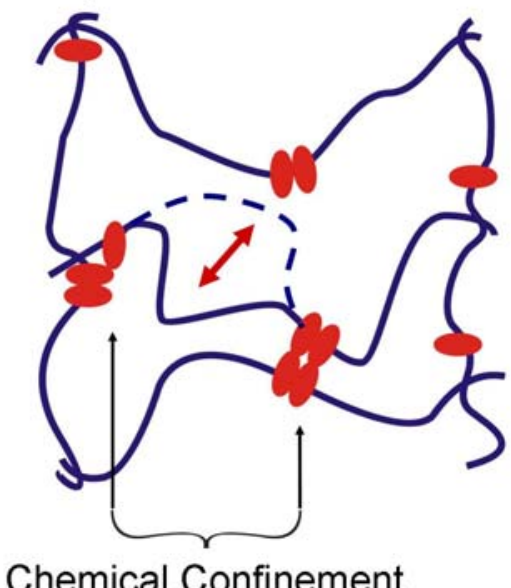

Abb. 6.6.1: Eine nicht statistische Verteilung der MAA-Einheiten in EMAA führt zu zusätzlichen Relaxationsmoden. Schematische Darstellung von: a) EMAA mit statistischer MAAVerteilung, b) mit nicht-statistischer Verteilung, c) der Netzwerkstruktur von EMAA mit statistischer MAA-Verteilung und d) mit nicht statistischer MAA-Verteilung und der daraus resultierenden „Chemical Confinement“-Relaxation

Ob eine Erhöhung des MAA-Gehalts zu einer Verschiebung des zusätzlichen Relaxationsmaximums zu tieferen oder höheren Temperaturen führt, hängt davon ab, welche Relaxation beeinflusst wird. In der Literatur sind dazu Beispiele für zusätzliche Relaxationen bei Copolymeren mit unterschiedlich steifen Monomereinheiten oder hervorgerufen durch das Einbringen von Quervernetzungen zu finden. Die vermuteten Auswirkungen auf das Relaxationsverhalten sind dabei nicht eindeutig. Es wird beschrieben, dass das Hinzufügen von steiferen Einheiten in eine Polyethylenkette [COW80, HBP96] oder das graduelle Ersetzen von Wasserstoff durch Chlor [AHL88] die zur $\gamma$-Relaxation führende Kurbelwellenbewegung stört. Aus einem Hinzufügen von immer mehr schlecht drehbaren Segmenten sollte eine Verschiebung der Relaxation zu höheren Temperaturen resultieren. Andere Argumentationen fußen auf Experimenten, bei denen durch eine Erhöhung des quervernetzten Anteils die Segment- 
bewegung ( $\alpha$-Relaxation) behindert wird [KKL05]. Ein höherer Quervernetzungsgrad führt dabei zu kürzeren, relaxierenden Segmenten und einer Verschiebung der zusätzlichen Relaxation zu tieferen Temperaturen. An dieser Stelle sei vorweggenommen, dass mit höherem MAA-Gehalt eine vergleichbare Verschiebung zu tieferen Temperaturen zu beobachten ist, da die zwischen den MAA-Blöcken liegenden Ethylenblöcke kürzer werden (vgl. Kap. 6.9). Dies rechtfertig die Vermutung, dass es sich um eine Segmentbewegung auf kleinen Längenskalen und nicht um eine erschwerte Kurbelwellebewegung handelt, wobei die bestehenden Wasserstoffbrückenbindungen nicht zerstört werden. Aufgrund der Vermutung, dass es sich um eine Relaxation von Ethylenblöcken zwischen MAA-Knotenpunkten handelt und in Anlehnung an die Begriffe des ,spatial confinement“ oder ,geometric confinement“(s.o.) wird diese zusätzliche Relaxationsmode als „chemical confinement“ oder cc-Relaxation bezeichnet.

\subsection{Die chemischen Eigenschaften der Copolymerproben}

Um in einem Copolymer gezielt cc-Relaxationen beobachten können, muss dieses hohen Qualitätsansprüchen genügen. Zunächst einmal wird eine homogene Probe benötigt, das heißt, dass es keine großen Fluktuationen innerhalb des Probenmaterials in Bezug auf chemische Zusammensetzung, Monomerverteilung oder Kettenlänge geben darf. Die Kettenlänge muss ausreichend lang sein, sodass es nicht zu einer Beeinflussung der Segmentbewegungen kommt. Darüber hinaus muss der Unterschied in der Monomerverteilung hinreichend stark durch Variation des Synthesedrucks beeinflussbar sein, um einen Unterschied zwischen den, bei hohem und bei niedrigem Druck synthetisierten, EMAA beobachten zu können. Damit die Proben untereinander vergleichbar sind, ist es erforderlich, die Monomerverteilung beeinflussen zu können, ohne die Konzentrationsverhältnisse wesentlich zu stören. Die Untersuchungsergebnisse im Hinblick auf die Erfüllung dieser Voraussetzungen sind in Kapitel 5.1 beschrieben.

\section{Chemische Zusammensetzung}

Einer der größten Vorzüge der verwendeten kontinuierlichen Hochdruck-HochtemperaturRührkessel-Herstellungsmethode (betrieben in der Arbeitsgruppe um Prof. Dr. M. Buback) ist, dass sie zu sehr homogenen Proben führt. Die in Kapitel 3.2 beschriebene Synthese verläuft dabei kontinuierlich und unter statischen Syntheseparametern, wenn das Reaktionsgleichgewicht eingestellt wurde. Somit sind keine großen Schwankungen der chemischen Zusammensetzung innerhalb einer Probe zu erwarten. Problematisch ist dagegen, dass stabile Bedingungen nur erreicht werden, wenn die Syntheseparameter gut aufeinander abgestimmt sind. Sollen zwei Proben mit annähernd gleichem MAA-Gehalt bei konstanter Temperatur unter zwei verschiedenen Synthesedrücken hergestellt werden, so ist dies nur möglich durch eine Anpassung des Initiatorflusses. Bei einem niedrigeren Druck ist dazu mehr Initiator nötig, damit die Reaktion nicht zum Erliegen kommt. Dem Effekt, dass der Umsatz bei niedrigerem Druck sinkt, wird somit entgegengewirkt. Dies ist wichtig, da eine Erhöhung des Umsatzes der beiden Monomere den MAA-Gehalt verschiebt, da die Aktivität der beiden Monomere nicht identisch ist [WIT99]. Zusätzlich wird bei einem höheren MAA-Anteil mehr Initiator benötigt (vgl. Abb. 5.1.5). Aus diesen Gründen ist eine Herstellung von Proben mit identischem MAA-Gehalt unter Variation des Synthesedrucks nicht trivial und bedarf genauerer Untersuchung. 
Die Ergebnisse der Untersuchung der Zusammensetzung fasst Abbildung 5.1.4 zusammen. Es ist deutlich zu erkennen, dass sich die MAA-Gehalte der einzelnen Herstellungsserien deutlich voneinander unterscheiden, die Werte aber auch eine deutliche Streuung aufweisen. Eine Konzentrationsbestimmung aller Proben liegt nur mittels NMR-Spektroskopie vor, da bei geringem MAA-Gehalt die Quantifizierung mittels ATR-FTIR keine positiven Werte lieferte und nicht aus allen Proben zur FTIR-Analyse in Transmission geeignete Folien hergestellt werden konnten. Die Problematik bei der ATR-FTIR-Auswertung beruht darauf, dass sich die Kalibration auf Messungen in Transmission beziehen. Die gemessenen Spektren in Abbildung 5.1.1 (Folien-FTIR) und Abbildung 5.1.2 (ATR-FTIR) ähneln sich sehr, sind aber bei genauerem Betrachten nicht identisch, z.B. in Bezug auf das Verhältnis von O-H-Untergrund zu Ethylen-Maxima bei Probe 3.4. Eine weitere Problematik bei der ATR-FTIR-Analyse ist die geringe Eindringtiefe. Kommt es zu einer bevorzugten Anlagerung von MAA-reichen Copolymereinheiten an das ATR-Prisma, so kann der gemessene MAA-Gehalt zu größeren Werten verfälscht sein. Bei den untersuchten Folien können Einschlüsse oder durch das Heißpressen entstandene Trübungen das Spektrum verfälschen.

Auch wenn die Fehlerquellen nicht genauer untersucht wurden, ist festzustellen, dass alle Analysemethoden einen gemeinsamen Trend aufweisen: Der MAA-Gehalt der einzelnen Proben unterscheidet sich innerhalb einer Serie bei derselben Analysemethode wenig (etwa $1 \mathrm{~mol} \%$ ) und deutlich zwischen den Serien (mehr als 2 mol\%). Damit ist eine Grundvoraussetzung an die EMAA-Proben erfüllt. Sie werden im Folgenden in Proben mit niedrigem, mittlerem und hohem MAA-Gehalt eingeteilt. Wann immer eine Auftragung als Funktion des MAA-Gehalts nötig ist, werden dazu die mittels NMR gewonnenen Konzentrationen genutzt, da mit dieser Methode für alle Proben ein Wert bestimmt werden konnte.

Eine Aussage über die genaue Verteilung von MAA- und Ethylen-Einheiten entlang der Copolymerkette ist zum Zeitpunkt der Fertigstellung dieser Arbeit noch nicht experimentell möglich. Eine Untersuchung des EMAA im nahen Infrarotbereich lieferte keine eindeutigen Ergebnisse und auch eine mehrdimensionale NMR-Analyse blieb sehr vage in ihrer Aussagekraft. Weitere mehrdimensionale NMR-Untersuchungen werden in [BS07] folgen.

\section{Kettenlängenverteilung}

Wie bereits erwähnt, ist eine weitere Voraussetzung an die Beschaffenheit eine hinreichende Länge der EMAA-Polymere, damit die Segmentbewegung nicht durch endständige Bereiche dominiert wird.

Die Ergebnisse der SEC-Analyse in den Abbildungen 5.1.6f zeigen, dass alle verwendeten Proben eine Molmassenverteilung besitzen, deren Maximum bei Werten von größer als $5000 \mathrm{~g} / \mathrm{mol}$ (Probe 3.12) liegen. Die Molmasse einer Ethyleneinheit beträgt etwa $28 \mathrm{~g} / \mathrm{mol}$ und die einer MAA-Einheit etwa $86 \mathrm{~g} / \mathrm{mol}$. Bei einem MAA-Gehalt von etwa $5 \mathrm{~mol} \%$ entspricht dies einem Maximum in der Molmassenverteilung von 162 Monomereinheiten. Dies wird als Polymerisationsgrad bezeichnet. Bei etwa $40000 \mathrm{~g} / \mathrm{mol}$ und $0.1 \mathrm{~mol} \%$ MAA-Gehalt liegt die Kettenlänge im Maximum der Molmassenverteilung bei Probe 12.4 bei etwa 1400 Monomereinheiten. Liegt der Polymerisationsgrad unterhalb von 100, ist eine deutliche Verschiebung der $\alpha$-Relaxation zu erwarten [SPE92]. Es kann also festgestellt werden, dass die mittels SEC bestimmte Molmassenverteilung Grund zur Annahme gibt, dass die Kettenlänge 
keinen wesentlichen Einfluss auf die Segmentbewegung hat. Bei den im Folgenden beschriebenen DSC- und DMA-Messungen wird dieser Punkt näher behandelt.

Bei Proben mit besonders hohen Molmassen sind ein oder mehrere weitere Maxima in der Molmassenverteilung bei Werten oberhalb $10^{5} \mathrm{~g} / \mathrm{mol} \mathrm{zu}$ beobachten. Es handelt sich dabei vermutlich um so genannte Langkettenverzweigungen, welche entstehen, wenn bei der Synthese ein Polymer mit radikalischem Ende mit einem weiteren Polymer reagiert. Dabei wird ersteres terminiert, und das zuvor nicht mehr aktive, zweite Polymer verfügt nun über eine Radikalfunktion. Somit kann dieses weiter wachsen und erreicht eine Molmasse, die aus der Molmasssenverteilung von Polymeren ohne Langkettenverzweigung herausragt. Dieser Vorgang kann sich auch mehrfach wiederholen, was ein Auftreten von mehr als einem zusätzlichen Maximum erklärt [BS07]. Diese Ereignisse sind besonders dann zu erwarten, wenn nur wenig Initiator eingesetzt wird. Ein solcher Vorgang wird als Radikal-Transfer-Reaktion bezeichnet, welcher in [BOX00] detailliert untersucht wird.

Über die Beschaffenheit der industriell hergestellten Proben lässt sich wenig aussagen, da das Herstellungsverfahren unbekannt ist. Es fällt lediglich auf, dass eine schärfere Molmassenverteilung als bei den übrigen Proben erreicht wurde. Insbesondere ist der Synthesedruck nicht bekannt, weshalb zunächst keine Aussagen über die Verteilung der Monomere im Copolymer gemacht werden können.

\subsection{Voruntersuchungen mittels Kalorimetrie}

Die Untersuchung der EMAA-Proben und der Homopolymere Polyethylen (PE) und Polymethacrylsäure (PMAA) mit der DSC bietet eine ergänzende Technik der Charakterisierung zur DMA. Aufgrund der Kühlung mittels Flüssigkühlers ist sie auf Temperaturen oberhalb $230 \mathrm{~K}$ beschränkt, kann aber andererseits den gesamten Übergang in die Flüssigkeit ausmessen. Dies ist bei der DMA nicht möglich, da keine flüssigen Proben im verwendeten Arbeitsmodus untersucht werden können. Zudem müssen die Proben für die DSC-Analyse nicht aufwendig in eine zylindrische Form gebracht werden. Dies vereinfacht die Untersuchung und erhöht die Reproduzierbarkeit. Die zu erwartenden Aussagen, die mittels DSC getroffen werden können, betreffen die Temperaturen und die Intensität der in diesem Bereich vorkommenden Relaxationen und Materialumwandlungen sowie deren Wärmetönung (endo- oder exotherm).

Bei der Untersuchung der Homopolymere (Abb. 5.2.1) kann zunächst festgestellt werden, dass beide nur über sehr wenige Stufen oder Übergänge im Wärmefluss bei Temperaturen bis $400 \mathrm{~K}$ verfügen. PE zeigt dabei ein scharfes Schmelzmaximum bei $394 \mathrm{~K}$, da es sich bei diesem PE mit niedriger Dichte um ein teilkristallines Material handelt [BEC06]. Der Glasübergang des nicht-kristallinen Anteils zeigt sich in Form einer flachen Stufe mit einem Onset bei 311 K. PMAA verfügt über für ein Polymer eher ungewöhnliche Eigenschaften. Zum einen ist es nach Händlerangaben zum Großteil kristallin und besitzt eine große Neigung Wasser aufzunehmen. Es kann sogar darin gelöst werden. Zum anderen liegt der Zersetzungspunkt unterhalb des Schmelzpunktes. Es ist daher nicht klar, ob der verschmierte Anstieg mit Maximum bei $385 \mathrm{~K}$ durch einen Glasübergang hervorgerufen wird oder ob es sich um eine Wechselwirkung mit darin enthaltenem Wasser handelt. Trotz der Lagerung des PMAA in einem gut verschlossenen Glasgefäß, ist eine Kontamination mit Wasser aber nicht auszuschließen. 
Über den Wärmefluss des PMAA lässt sich aussagen, dass bis auf den breiten Anstieg keine weiteren Relaxationen zu erkennen sind und sich die Zersetzung des Materials in einem sich beschleunigenden Anstieg oberhalb von $420 \mathrm{~K}$ zeigt.

Das EMAA zeigt deutlich mehr Struktur im Wärmefluss, verglichen mit den Homopolymeren, insbesondere bei Proben die bei niedrigem Druck synthetisiert wurden. Die Nomenklatur der Maxima erfolgt anhand der Probe 3.12 (vgl. Abb. 5.2.4), welche die meisten Abweichungen von einem linearen Wärmefluss zeigt. Diese geht von der als Glasübergang identifizierten Relaxation aus, deren Onset als $T_{\mathrm{G}}$ und Maximum als $T_{\alpha}$ bezeichnet werden. Das Maximum des Übergangs in die Flüssigkeit erhält die Bezeichnung $T_{\alpha 2}$, Maxima in der Temperatur unter dem Glasübergang liegende Übergänge werden willkürlich mit den griechischen Buchstaben $\beta$ und $\gamma$ bezeichnet. Diese Bezeichnungen beziehen sich nicht auf die in Kapitel 2.6 beschriebenen Prozesse. Ob Seitengruppenbewegungen eine Rolle spielen, kann nicht abschließend geklärt werden. Es ist jedoch als sicher anzunehmen, dass in diesem Temperaturbereich nicht die als Kurbelwellenbewegung beschriebene, für PE charakteristische Relaxation vorkommt. Dies ist auch aus dem Fehlen entsprechender Stufen oder Maxima im PE-Wärmefluss zu schließen. Was jedoch direkt auffällt ist, dass ein $\beta$-Maximum bei mit niedrigem Synthesedruck hergestellten Proben weitaus deutlicher zu Tage tritt und dass es auch mit steigendem MAA-Gehalt anwächst. Bei Probe 3.12 mit hohem MAA-Gehalt ist noch ein zusätzliches $\gamma$ Maximum zu beobachten, bei Probe 12.4 mit niedrigem MAA-Gehalt manifestiert sich das $\beta$ Maximum nur sehr undeutlich. Dies sind deutliche Indizien dafür, dass bei niedrigerem Druck synthetisiertes EMAA über zusätzliche Relaxationsmoden verfügt. Studien an reinem PE mit niedriger Dichte haben gezeigt, dass die Anzahl der Verzweigungen nur sehr schwach druckabhängig ist und somit nicht ausreicht, um die beobachteten Phänomene zu erklären [LKS82]. Eine weitere Beobachtung ist, dass die Intensität des $\alpha 2$-Maximums mit steigendem MAAGehalt abnimmt. Dieser Effekt ist bei niedrigem Synthesedruck stärker als bei hohem. Dies ist dadurch zu erklären, dass eine Erhöhung des MAA-Anteils die Bildung von kristallinen PEBereichen im EMAA erschwert, insbesondere wenn diese in Blöcken auftreten. Dies führt zu einem höheren Anteil von amorphen Bereichen, wodurch das $\alpha$-Maximum an Intensität gewinnt.

Die Notwendigkeit der Einhaltung von exakten Temperaturbedingungen während der Synthese ist im DSC-Signal der Probe 1.2 (vgl. Abb. 5.2.3) zu sehen. Diese war um mehr als $10 \mathrm{~K}$ zu niedrig (vgl. 5.1.2). Dies manifestiert sich im Wärmefluss durch einen Kurvenverlauf, welcher eher der bei niedrigem Druck synthetisierten Probe 2.10 als der ebenfalls bei hohem Druck synthetisierten Probe 2.2 entspricht. Zusätzlich ist das $\alpha 2$-Maximum in zwei Maxima aufgespaltet. Dies spricht dafür, dass eine Herabsetzung der Temperatur ebenfalls zu einer weiniger statistischen Verteilung führt und somit ähnlich wirkt wie eine Reduktion des Synthesedrucks. Eine mögliche Erklärung der Zunahme in der Höhe des $\alpha 2$-Hauptmaximums bieten Studien an PE mit niedriger Dichte in denen gezeigt werden konnte, dass eine Herabsetzung der Temperatur die Zahl der Kurzkettenverzweigungen reduziert, wodurch eine Bildung von kristallinen Bereichen vereinfacht wird [LKS82].

Es zeigt sich auch bereits in den kalorimetrischen Untersuchungen, dass ein Vergleich der synthetisierten EMAA-Proben mit den industriellen Proben nur eingeschränkt möglich ist. Beide zeigen lediglich $\alpha$ - und $\alpha 2$-Maxima, ein erstes Indiz dafür, dass sie über keine zusätzli- 
che charakteristischen Längenskalen im Bereich der Segmentbewegung in diesem Temperaturintervall verfügen. Ein drittes, scharfes Maximum mit geringer Intensität oberhalb $376 \mathrm{~K}$ ist vermutlich auf eine Kontamination der Probe oder der Probenkammer mit Wasser zurückzuführen, welches bei diesen Temperaturen verdampft.

Wie eingangs erwähnt, ist die DSC besonders zur Voruntersuchung dahingehend geeignet, ob die Kettenlänge ausreichend groß ist, um die Segmentbewegung nicht zu beeinflussen. Hier ist ein positives Ergebnis erzielt worden, wie in Abbildung 5.2.6 ersehen werden kann. Aufgetragen ist die Molmassenabhängigkeit der charakteristischen Temperaturen der $\alpha$ - und der $\alpha 2-$ Relaxation (Position des Maximums), sowie der Onset zur $\alpha$-Relaxation, der bei den DSCUntersuchungen als $T_{\mathrm{G}}$ bezeichnet wird. Es ist mit steigender Molmasse ein deutlicher Anstieg der Temperatur des Auftretens des a2-Maximums zu beobachten. Dies spricht für eine deutliche Kettenlängenabhängigkeit. Die eingezeichnete Gerade ist keine angepasste Ausgleichsgerade, sondern als Abschätzung der unteren Grenze der Steigung zu werten. Dabei fällt auf, dass die Punkte der Maxima von bei niedrigem Druck synthetisierten Proben in der Auftragung links liegen. Verknüpft mit der bereits festgestellten Tatsache, dass auch die Intensität der Maxima der bei niedrigem Druck synthetisierten Proben kleiner ist, kann unter der Annahme einer weniger statistischen Verteilung der MAA-Einheiten gefolgert werden, dass benachbarte MAA-Gruppen eine Bildung von kristallinen Bereichen behindern. Bei der Betrachtung nach Synthesedrücken getrennt ist festzustellen, dass insbesondere eine Ausgleichskurve durch die Positionen der bei hohem Druck synthetisierten Proben deutlich steiler verlaufen könnte. Darüber hinaus fällt auf, dass sich auch in diesem Zusammenhang die industriellen Proben tendenziell wie Hochdruckproben verhalten.

Aus den gemessenen Daten ist keine Abhängigkeit der $\alpha$-Relaxation von der Kettenlänge zu erkennen. Sowohl die Temperaturen der Maxima $T_{\alpha}$, als auch die der Onsets $T_{\mathrm{G}}$ schwanken stark um die eingezeichnete horizontal verlaufende Linie. Der Trend ist allerdings eher fallend als steigend. Somit ist keine signifikante Beeinflussung der Segmentbeweglichkeit von der Kettenlänge festzustellen. Besonders interessant ist auch, dass es keine Abhängigkeit vom Synthesedruck zu geben scheint. Es ist zu vermuten, dass ein eventuell vorliegender kristalliner Anteil die Bewegung der amorph vorliegenden Ketten kaum beeinflusst. Eine geringe Temperaturabnahme beider Kenngrößen ist nur dann ersichtlich, wenn die industriell hergestellten Proben herausgenommen werden. Diese scheinen zu höheren Temperaturen verschoben zu sein, was auch mit der unbekannten thermischen und mechanischen Vorgeschichte der Proben zusammenhängen kann.

Um die Effekte der thermischen Vorgeschichte zu untersuchen, sind zwei Proben der MAAreichen Serie 3, welche bei sehr unterschiedlichen Drücken synthetisiert wurden, in mehreren Stufen aufgeheizt worden (Abb. 5.2.7f). Durch das Verpressen der möglichst voll gefüllten Probenpfännchen wird eine anfängliche, makroskopische Deformation des Probenmaterials erzeugt. Somit sollte ein wiederholtes Durchlaufen von Relaxationen, die zu einem Erweichen der Probe führen und irreversible Anteile haben, Unterschiede im Vergleich zum ersten Durchlaufen aufweisen. Auch bei tieferen Temperaturen liegende Relaxationen können infolgedessen beeinflusst werden. Bei beiden Proben ist zu erkennen, dass ein Durchlaufen des $\gamma-$ Maximums (soweit erkennbar) und des darauf folgenden $\beta$-Maximums zu keiner Veränderung des Verlaufs des Wärmeflusses führen. Die Kurvenform der in einem Aufheizen gemes- 
senen Referenz wird qualitativ reproduziert. Es kommt lediglich zu einer reproduzierbaren Störung beider Proben im Bereich um 266 K. Auch dabei könnte es sich um Wasser handeln. Ein zunächst exothermer Ausschlag in Abbildung 5.2.8 kann dabei von Wasser am oder im Referenztiegel stammen, wenn zwischen beiden eine Temperaturdifferenz besteht. Eine Verschiebung der Spektren um einen in erster Nährung linearen Versatz zwischen Referenz und gestuftem Heizen ist durch eine Veränderung der Basislinie zu erklären. Dazu reicht schon ein anderes Verhältnis von Proben- zu Tiegelgewicht aus.

$\mathrm{Zu}$ Veränderungen im Wärmefluss kommt es erst, wenn dass $\alpha$-Maximum im vorangegangenen Heizzyklus überschritten wurde. Dies führt dann zu einem breiteren $\alpha$-Maximum beim folgenden Aufheizen, welches das $\beta$-Maximum sogar überdecken kann. Darüber hinaus ist vor dem Erreichen des $\alpha 2$-Maximums ein weiteres zu erkennen. Ob sich dabei die $\alpha$ - oder die $\alpha 2$-Relaxation in zwei Vorgänge aufspaltet, ist schwer zu entscheiden.

Zusammenfassend lässt sich festhalten, dass bereits die kalorimetrische Analyse Unterschiede zwischen den synthetisierten Proben aufzeigt, welche über einen reinen Kettenlängeneffekt hinausgehen. Bei niedrigerem Druck synthetisierte Proben, bei denen von einer weniger statistischen Verteilung von MAA-Einheiten ausgegangen wird, sind deutlicher ausgeprägte zusätzliche Relaxationen unterhalb der $\alpha$-Relaxation zu beobachten. Auch die Lage der $\alpha 2-$ Relaxation ist druckabhängig. Eine Änderung der mikroskopischen Eigenschaften hat also zu einer deutlichen Änderung der makroskopischen, kalorimetrischen Eigenschaften geführt. Somit kann bereits hier geschlossen werden, dass sich diese Effekte auch in den mechanischen Eigenschaften widerspiegeln sollten.

\subsection{Mechanische Eigenschaften und "Chemical Confinement"}

\section{Verhalten oberhalb Zimmertemperatur und Effekte der Vorgeschichte}

Die erste Beobachtung bei der Untersuchung der synthetisierten EMAA-Proben ist, dass Proben mit hohem MAA-Gehalt deutlich weicher sind als solche mit niedrigem MAA-Gehalt. Dies ist nicht weiter überraschend, da mit einer Erhöhung des MAA-Gehalts ein präparativ bedingtes Absinken der Kettenlänge (Abb. 5.1.6) einhergeht. Dies führt dazu, dass die $\alpha 2-$ Relaxation bei niedrigeren Temperaturen liegt und bereits in den kalorimetrischen Untersuchungen bei höherem MAA-Gehalt dichter zur $\alpha$-Relaxation ist (Abb. 5.2.6). Interessanter ist nun, ob eine Veränderung der mechanischen Moduln durch eine Variation des Synthesedrucks bei annähernd gleichem MAA-Gehalt zu beobachten ist.

Hierbei wird deutlich, dass die Bestimmung des Absolutwerts des Speichermoduls stark von der thermischen und mechanischen Vorgeschichte des Materials abhängig ist. Dies zeigt sich deutlich bei dem Versuch, denselben Testzylinder ein zweites Mal spektroskopisch zu untersuchen. Wird der Heizzyklus direkt ein zweites Mal durchfahren, nachdem der erste Lauf bis zum Beginn der $\alpha 2$-Relaxation ausgeführt worden ist und nach dem ein Abkühlen ohne anliegende Kräfte erfolgt ist, ist mehr als eine Verdreifachung des Speichermoduls festzustellen (Abb. 5.3.1). Im Gegensatz dazu ist ein deutlich reduzierter Speichermodul zu beobachten (Abb. 5.3.2), wenn nach dem ersten Aufheizen bis zu Beginn der $\alpha 2-$ Relaxation und dem anschließenden Abkühlen ohne anliegende Kräfte die Probe zunächst von Stempel und Bodenplatte getrennt wird. Dies wurde bei Zimmertemperatur vor dem Einkühlen zum zweiten Durchlauf durchgeführt. Es gibt zwei mögliche Erklärungen für diese Beobachtung: Zum ei- 
nen könnte es sein, dass danach der Stempel nicht auf der gesamten Fläche des Testzylinders auflag. Zum anderen kann es sich um einen echten Alterungseffekt handeln, hervorgerufen durch eine „Verjüngung“ der Polymerausrichtung durch das Kaltverformen beim Ablösen der Probe von Stempel und Bodenplatte. Was gegen Erstens und für ein Alterungssignal oder eine Mischform von beidem spricht ist, dass die stärkste Änderung der Sondenstab-Position, also der momentane Abstand des Stempels zur Bodenplatte, nicht mit der stärksten Abnahme der Amplitude bei etwa $325 \mathrm{~K}$ zusammen fällt, wie in Abbildung 6.9.1 gezeigt. Dies entspricht Punkt Zwei der in Kapitel 6.5 beschriebenen Annahmen zum Ablauf eines DMAExperiments: Ein nicht moduliertes Signal entspricht bei $5 \mathrm{~K} / \mathrm{min}$ Heizrate einer längeren charakteristischen Zeitskala als die inverse Frequenz von $5 \mathrm{~Hz}$. Folglich erfolgt die Änderung der dynamischen Größe der Amplitude bei höheren Temperaturen. Würde lediglich ein Grad am Rand der Probe zerdrückt, so sollte die Amplitude gleichzeitig mit der SondenstabPosition abfallen. Der beobachtete Prozess des so genannten „strain hardenings“ [STR77] geht mit einem Maximum im Speicher- und im Verlustmodul in Abbildung 5.3.2 einher. Derselbe Effekt ist in Abbildung 5.3.1 beim ersten Aufheizen zu beobachten und führt zu einer deutlich härteren Probe beim zweiten Durchlauf ohne zwischenzeitiges Lösen der Probe von Stempel und Bodenplatte. Der dort beobachtete Anstieg des Speichermoduls bei niedrigeren Temperaturen ist auf ein Einsetzen der Messbarkeit von Amplitude und Phasenwinkel zurückzuführen.

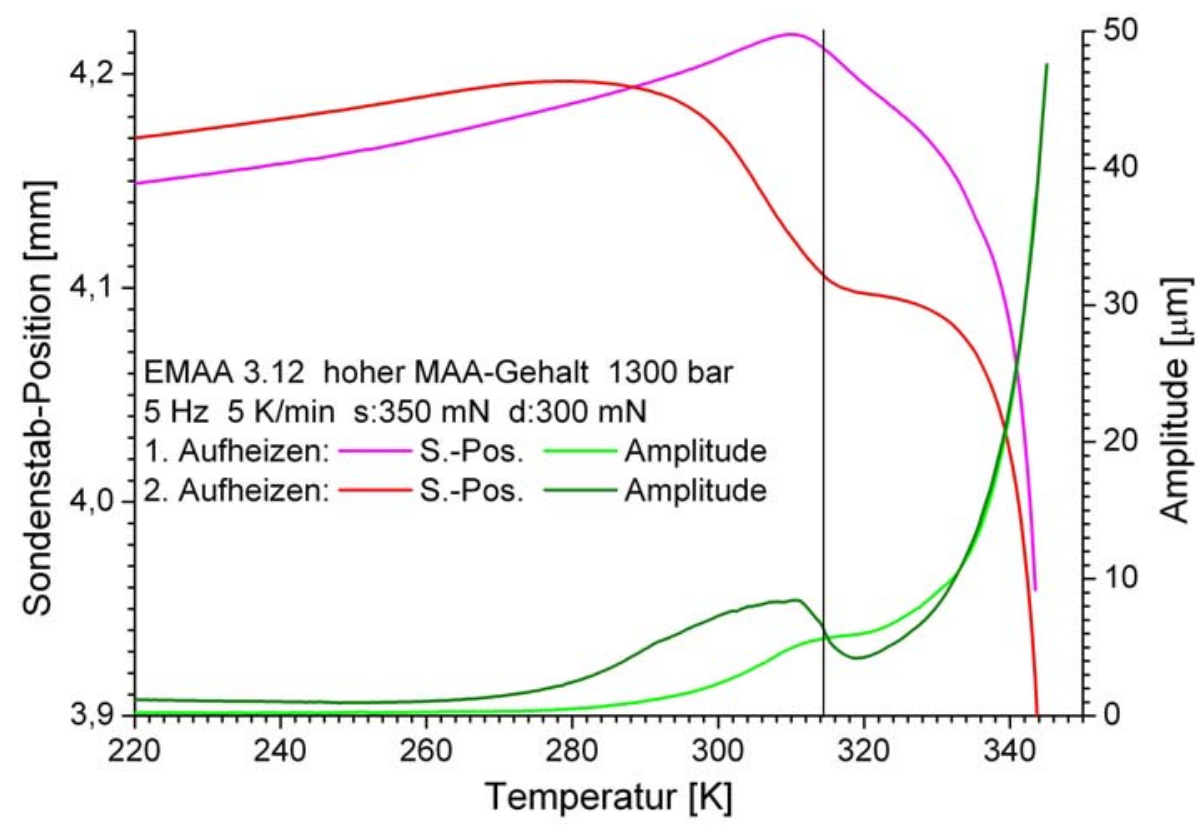

Abb. 6.9.1: Auftreten des Alterungseffekt in Sondenposition und Amplitude; Wendepunkt im Amplitudensignal (schwarze Linie) fällt nicht mit jenem des Positionssignals zusammen.

Eine genau definierte Präparation eines konstanten „Probenalters“ ist mit der genutzten Methode des Heißpressens, wie in Kapitel 3.3 beschrieben, nur sehr schwer möglich. Es wurde stets dasselbe Gewicht verwendet und bei ähnlichen Temperaturen gepresst. Da sich aber die Lage der $\alpha 2$-Relaxation von Probe zu Probe verändert und keine Kontrollmöglichkeit des aktuellen Probenvolumens gegeben ist, kann die Vorkompression der Probe nicht eingestellt werden. Wünschenswert wäre die Herstellung von blasenfreien EMAA-Folien mit einer Stär- 
ke von etwa $1 \mathrm{~mm}$. Diese könnten ausgelagert und somit entspannt werden. Daraus präparierte Streifen sollten sich gut auf ihre mechanischen Tieftemperatureigenschaften in einem Biegeexperiment untersuchen lassen. Leider ist die Präparation von Folien aus EMAA sehr schwierig. Aufgrund dieser Komplikationen ist die folgende Betrachtung auf den geometriefreien $\tan \delta$ von möglichst, ,jungen“ Proben beschränkt. Auf diese Weise ist eine Vergleichbarkeit der Relaxationsspektren in einem möglichst großen Temperaturintervall möglich.

\section{Zusätzliche Relaxationsmode bei tiefen Temperaturen}

Wie bei den DSC-Untersuchungen ist es auch hier sinnvoll, zunächst die Relaxationsspektren der Homopolymere (Abb. 5.3.3) zu betrachten. PE zeigt eine $\gamma$-Relaxation mit einem Maximum bei $155 \mathrm{~K}$ (vgl. Kap. 2.6) und oberhalb $300 \mathrm{~K}$ einen leicht kurvigen Verlauf, welcher im Vergleich mit den DSC-Untersuchungen als $\alpha$-Relaxation der amorphen Bereiche gedeutet wird. Langkettenverzweigungen könnten hier zu einem weiteren Maximum führen. Das mit Wasser versetzte PMAA zeigt lediglich einen Anstieg zu einem breiten Maximum bei etwa $235 \mathrm{~K}$, welches sich in halblogarithmischer Auftragung als Schulter im ansonsten annähernd linearen Anstieg zeigt.

EMAA der Serie 12 mit niedrigem MAA-Gehalt von 0,1 mol\% - 0,2 mol\% zeigt eine Kombination aus beiden Verhalten: Eine $\gamma$-Relaxation mit Maximum bei $159 \mathrm{~K}$ sowie ein breites Maximum bei $277 \mathrm{~K}$, welches als $\alpha$-Relaxation bezeichnet wird. Letztere liegt zwischen den $\alpha$-Relaxationen der Homopolymere, aber deutlich unterhalb des mittels DSC bestimmten Wertes von $T_{\alpha}$, DSC $=325 \mathrm{~K}$, welcher im Temperaturbereich des Anstiegs der SondenstabPosition in Abbildung 6.9.2 liegt. Grundsätzlich sollte aber zuerst ein Effekt im statischen Signal der Sondenstab-Position zu beobachten sein und danach im dynamischen Signal des $\tan \delta$. Dies legt die Vermutung nahe, dass eine Behinderung der Segmentbewegung nicht auszuschließen ist. Ein Kettenlängeneffekt kommt nicht in Frage, da diese Verschiebung des $\alpha$-Maximums im $\tan \delta$-Signal am deutlichsten bei Serie 12 beobachtet wird, welche die größten Kettenlängen besitzt (Abb. 5.3.9). Eine mögliche Erklärung ist, dass die von der Vorbehandlung abhängende Größe der kristallinen Bereiche die Segmentbewegung beeinflusst. Dafür spricht, dass die Intensität des $\alpha 2$-Maximums im DSC-Signal bei den Proben am höchsten ist (Abb. 5.2.2f), die gleichzeitig den geringsten, eine Kristallisation potenziell störenden MAA-Gehalt aufweisen.

Eine weitere Interpretationsmöglichkeit neben der Behinderung der Segmentbeweglichkeit ist das Auftreten von Langkettenverzweigungen, welche die $\alpha$-Relaxation zu tiefen Temperaturen verbreitern können. Dafür dass dies nicht der dominierende Effekt ist, spricht die Tatsache, dass diese Verschiebung auch bei EMAA 12.4 auftritt. Deren zweites Maximum in der Kettenlängenverteilungsfunktion (Abb. 5.1.6) ist aber zumindest vergleichbar mit Probe 1.2, bei der wiederum keine Verschiebung von $T_{\alpha}$ zu beobachten ist (Abb. 5.3.9).

Durch den vermuteten Einfluss von kristallinen Bereichen kommt Serie 12 eine Sonderstellung zu. Ein ähnliches Verhalten zeigt auch die industrielle Probe mit 5,5 mol\% MAA-Gehalt (vgl. Abb. 5.2.5 + 5.3.9). Ohne diese drei Punkte ist in Abbildung 5.3.9 eine unwesentliche Abhängigkeit der Temperatur des $\alpha$-Maximums von der Kettenlänge zu erkennen. Relaxationen auf noch kürzeren Längenskalen sollten davon unbeeinflusst sein. 


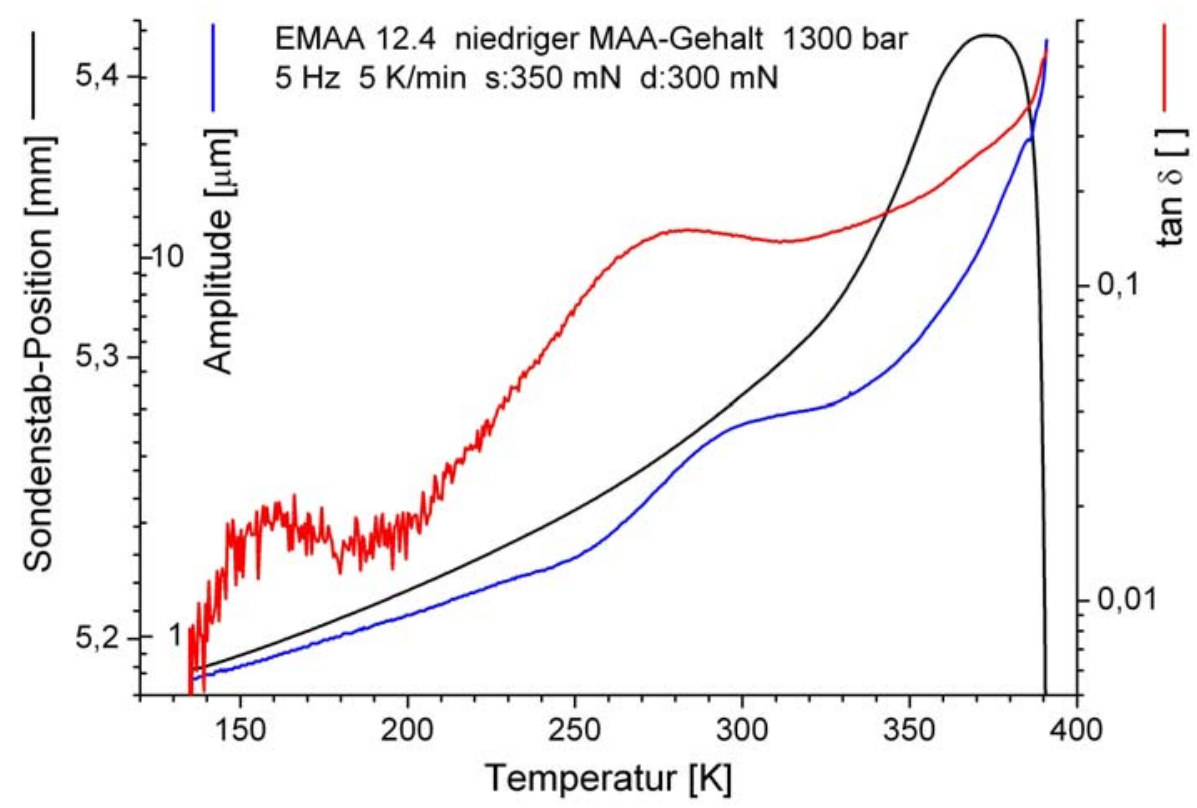

Abb. 6.9.2: Relative Position von statischen und dynamischen Größen der Messung in Abb.

\section{3 .4}

Es zeigt sich, dass bei niedrigem Druck synthetisierte Proben generell besser zu messende Relaxationsspektren aufweisen. Ferner kommt bei diesen Proben bei mittlerem und hohem MAA-Gehalt eine zusätzliche Relaxationsmode hinzu, welche den bei hohem Druck synthetisierten Proben fehlt (Abb. 5.3.5f). Auch im Verlauf des $\tan \delta$ der industriell hergestellten Proben (Abb. 5.3.7) ist keine zusätzliche Relaxationsmode auszumachen. Ihr Verhalten ähnelt eher dem von Hochdruckproben (vgl. Kap. 6.8). Diese zusätzliche Relaxationsmode wird in Kapitel 5.3 als $\beta$-Relaxation bezeichnet. Dafür, dass es sich dabei wirklich um eine ccRelaxation (vgl. Kap. 6.6) handelt, spricht die Verschiebung des cc-Maximums mit steigendem MAA-Gehalt, wie in Abbildung 5.3.8 gezeigt: Eine Erhöhung des MAA-Gehalts führt zu einer höheren Zahl von MAA-Blöcken auf der EMAA-Kette, wodurch die dazwischen liegenden PE-Segmente verkürzt werden. Dies verschiebt die cc-Relaxation zu niedrigeren Temperaturen. Dies spricht deutlich für die Gültigkeit der Arbeitshypothese. Weitere Belege könnten andauernde DES-Messungen (vgl. Kap. 3.9) erbringen. Darüber hinaus ist eine genauere Strukturaufklärung mittels mehrdimensionaler NMR (vgl. Kap. 3.8) wünschenswert [BS07]. 


\section{Zusammenfassung}

Das Ziel dieser Arbeit war die Untersuchung von amorphen Metalllegierungen und Copolymeren mit der Absicht, deren Relaxationsspektrum so zu beeinflussen, dass bisher nicht zweifelsfrei belegte Relaxationsmoden einzeln nachweisbar werden.

Im Fall der metallischen Gläser galt das Hauptaugenmerk der sekundären, langsamen $\beta$ Relaxation, welche ein universelles Phänomen des Glasübergangs zu sein scheint. Es galt diese durch gezielte Wahl der Messparameter unter Berücksichtigung der Vorgeschichte von der primären $\alpha$-Relaxation zu trennen. Besonderes Interesse erregte dabei ein Alterungsprozess beim ersten Aufheizen der amorphen Bänder, den es in das Relaxationsspektrum einzuordnen galt.

Bei den Copolymeren dagegen wurde eine wohldefinierte Synthesemethode genutzt, um eine zur $\alpha$-Relaxation führende Segmentbewegung bei tieferen Temperaturen so zu beschränken, dass es zu einer so genannten Chemical-Confinement-Relaxation (cc-Relaxation) kommt.

\section{Universelles Phänomen des Glasübergangs: Die langsame $\beta$-Relaxation}

Die Beobachtung von sekundären Relaxationen bei organischen Proben ist keine Seltenheit [LL05]. Es bleibt bei Systemen mit komplexer Molekülstruktur jedoch stets die Frage nach dem Ursprung der beobachteten Relaxationen. Zur Klärung der Frage der Existenz der langsamen $\beta$-Relaxation als universelles Phänomen des Glasübergangs ist es deshalb nötig, ein möglichst einfaches Glas zu untersuchen. Amorphe metallische Legierungen bieten sich dazu an, da ihre atomare Struktur als ungeordnetes System harter Kugeln mit attraktiven und repulsiven Wechselwirkungen beschrieben werden kann [FFM03]. Aufgrund der guten Leitfähigkeit ist eine Untersuchung des Relaxationsspektrums mittels dielektrischer Spektroskopie (DES) ausgeschlossen. Deshalb wurde die Untersuchungsmethode der mechanischen Spektroskopie bei fester Frequenz und variabler Temperatur angewendet. Zur Anpassung der elastischen Moduln wurde eine Havriliak-Negami-Funktion benutzt, welche durch die Parametrisierung der Relaxationszeit in Form einer Vogel-Fulcher-Tammann-Funktion in den Temperaturraum transformiert wurde [RÖS04]. Dabei muss die Gültigkeit des Zeit-TemperaturSuperpositionsprinzips angenommen werden. Erste Indizien für die Existenz einer langsamen $\beta$-Relaxation lieferten bereits Messungen an $\mathrm{Zr}_{65} \mathrm{Al}_{7,5} \mathrm{Cu}_{27,5}$ mit dem Doppel-PaddelOszillator (DPO). Dies konnte daraus geschlussfolgert werden, dass die Anpassung der Daten unter Annahme einer einzelnen $\alpha$-Relaxation und dem darunter liegenden nahezu konstanten Verlust (NCL) nicht möglich war [RSL04]. Mit dem Übergang von niedrigen Heizraten (ca. $0,2 \mathrm{~K} / \mathrm{min}$ ) und hohen Frequenzen $(5,4 \mathrm{kHz})$, wie sie für Experimente am DPO typisch sind, hin zu höheren Heizraten (bis $20 \mathrm{~K} / \mathrm{min})$ und niedrigen Frequenzen $(1-10 \mathrm{~Hz}$ ) bei der dynamisch-mechanischen Analyse (DMA), verringert sich das Problem der frühen Kristallisation.

Das Auftreten der langsamen $\beta$-Relaxation im eher starken Glasbildner $\mathrm{Zr}_{65} \mathrm{Al}_{7,5} \mathrm{Cu}_{27,5}$ und auch im fragileren $\mathrm{Pd}_{77,5} \mathrm{Cu}_{6} \mathrm{Si}_{16,5}$ legt denn Schluss nahe, dass es sich hierbei um ein universelles Phänomen und somit einen festen Bestandteil des Glasübergangs handelt.

Die Sichtbarkeit der langsamen $\beta$-Relaxation als Tieftemperaturflanke (,excess wing“) der $\alpha$ Relaxation hängt wesentlich von der Wahl der Messparameter ab und wird auch durch ein vorheriges Auslagern beeinflusst. Das Auftreten ist besonders ausgeprägt bei hohen Heizra- 
ten, ausreichend hohen Frequenzen und rasch abgeschreckten Proben, die nicht weiter ausgelagert wurden. Die Erklärung hierfür ist, dass es bei diesen Experimenten nicht nur zwei Relaxationen gibt, sondern auch zwei charakteristische Beobachtungszeiten: Jene die zur Temperaturerhöhung benötigt wird und mit der Heizrate verknüpft ist, und jene der Schwingungsdauer der Spektroskopie. Es kann somit zu bis zu vier Resonanzen kommen, in denen mit anderen Worten das Deborah-Kriterium erfüllt ist. Darüber hinaus ist jede Art von Überlagerung der vier Resonanzen durch ein Koppeln untereinander möglich. Von der $\alpha$-Relaxation ist dabei stets nur der Anfang beobachtbar, da die Kristallisation einsetzt, wenn die Beweglichkeit im Glas steigt. Mit diesem einfachen Erklärungsansatz ist eine umfassende Deutung der Verschiebung der Temperaturen des Auftretens von Relaxationen beim Wechsel von DPO zu DMA bei verschiedenen Messparametern in DMA-Serien und im Vergleich zu Ultraschallexperimenten [KAH06] möglich. Auch das Auftreten von Alterungseffekten (,,physical aging“) und eine umgekehrt Nicht-Newton'sche Kraftabhängigkeit der Viskosität kann so erklärt werden. Diese Effekte sind bei Polymeren seit langem Gegenstand der Forschung [STR77]. Im Fall der metallischen Gläser gibt es zu diesem Thema nur wenige experimentelle Studien [LXG90, PPS00]. Jüngste Messungen deuten darauf hin, dass auch bei metallischen Gläsern eine Verjüngung möglich ist [KAH06].

In dieser Arbeit konnte gezeigt werden, dass es die langsame $\beta$-Relaxation ist, die zu den Alterungsprozessen bei tieferen Temperaturen führt. Dabei läuft die langsame $\beta$-Relaxation auf der zur Heizrate gehörenden charakteristischen Beobachtungszeit ab.

Die Bestätigung hierfür wird durch spektroskopische Messungen an PdCuSi und ZrAlCu erbracht, bei denen die Heizrate in einem großen Bereich variiert wird. Dabei zeigt sich, dass sich die Temperatur, bei der die langsame $\beta$-Relaxation auftritt, viel stärkerer mit der Heizrate verschiebt als die der $\alpha$-Relaxation. Dies erlaubt die Interpretation, dass die langsame $\beta$ Relaxation beim ersten Aufheizen zweimal bei sehr verschiedenen Temperaturen in Erscheinung tritt: Als Alterungseffekt auf der Zeitskala des Aufheizens und als „excess wing“ der $\alpha$ Relaxation auf der Zeitskala der Schwingungsdauer.

Das Prinzip der Zeit-Temperatur-Superposition ist nur gültig, wenn keine Alterung oder Verjüngung des Systems von statten geht. Da der Gleichgewichtszustand der elastischen Moduln aber temperaturabhängig zu sein scheint [KAH06], ist diese Voraussetzung nicht erfüllt. Der Einfluss auf den Verlauf des Elastizitätsmoduls ist aber klein, wenn die charakteristische Zeit des Heizens deutlich länger ist als die Schwingungsdauer der Spektroskopie und kann sich bei zuvor ausgelagerten Proben weiter verringern lassen. Der Widerspruch zwischen der Annahme der alleinigen Existenz der $\alpha$-Relaxation und den Messungen an metallischen Gläsern bleibt weiter bestehen. Das Auftreten eines Alterungsvorgangs verstärkt hier sogar die Notwendigkeit einer sekundären Relaxation als zentrales Phänomen des Glasübergangs.

\section{Chemical-Confinement der Segmentbewegung in Copolymeren}

Um zeigen zu können, dass sich eine Segmentbewegung im Polymer gezielt durch das Einbringen von chemischen Knotenpunkten beschränken lässt, welche sich in Form einer zusätzlichen Relaxationsmode bei tiefen Temperaturen zeigt (cc-Relaxation), mussten zunächst hohe Materialanforderungen erfüllt werden. Das Verfahren der kontinuierlichen HochdruckHochtemperatur-Rührkessel-Synthese [WIT99] erwies sich dabei als gut geeignet, um das 
Copolymer Poly(Ethylen-co-Methacrylsäure) (EMAA) mit den benötigten Eigenschaften herzustellen. Dazu gehört neben der Homogenität der Probe eine ausreichend lange Kettenlänge, sodass diese nicht zu einer Beeinflussung der Segmentbewegung führt. Darüber hinaus besitzt EMAA, welches bei relativ niedrigem Druck von 1300 bar synthetisiert worden ist, eine weniger statistische Verteilung des Comonomers Methacrylsäure (MAA) entlang der Ketten, als bei hohem Synthesedruck (ca. 2000 bar) hergestelltes EMAA. Auf diese Weise kann untersucht werden, wie die MAA-Verteilung die Materialeigenschaften beeinflusst, ohne dass sich der mittlere MAA-Gehalt des Copolymers signifikant ändert. Es zeigt sich in der Tat, dass eine derartige Manipulation der mikroskopischen, chemischen Struktur zu einer Veränderung der makroskopischen, mechanischen Eigenschaften führt: Die bei niedrigem Synthesedruck hergestellten Proben zeigen einen höheren Verlustmodul als jene, die bei höherem Druck synthetisiert wurden. In Experimenten mittels differentieller Kalorimetrie (DSC) zeigt sich darüber hinaus, dass in den Niedrig-Druck-Proben mehr oder zumindest stärker ausgeprägte Übergänge stattfinden.

Im Rahmen dieser Arbeit wurde die folgende Arbeitshypothese aufgestellt: Liegt eine weniger statistische Verteilung der MAA-Einheiten entlang der Polymer-Kette vor, so kommt es zur Bildung von kurzen Blöcken, wobei die über Wasserstoffbrückenbindungen wechselwirkenden MAA-Blöcke feste Knotenpunkte darstellen, zwischen denen die beweglicheren EthylenEinheiten eine Segmentbewegung durchführen können. Diese Behinderung der zur $\alpha$ Relaxation führenden Segmentbewegung führt zu einer zusätzlichen cc-Relaxation bei tieferen Temperaturen.

Die cc-Relaxation konnte in DMA-Untersuchungen nachgewiesen werden, wobei diese nur an Proben auftritt, welche aufgrund eines niedrigen Synthesedrucks eine weniger statistische MAA-Verteilung aufweisen. Die Arbeitshypothese wird ebenfalls durch die Konzentrationsabhängigkeit der Position des Maximums der cc-Relaxation bestätigt: Bei einem höheren MAA-Gehalt liegen kürzere Ethylen-Segmente zwischen häufiger vorkommenden MAABlöcken vor. Eine Verkürzung der relaxierenden Segmente führt zu einer Verschiebung zu tieferen Temperaturen, was experimentell belegt werden konnte.

Im Gegensatz zur Untersuchung der langsamen $\beta$-Relaxation im System der metallischen Gläser beginnt bei den Copolymeren jede Untersuchung bei Temperaturen, bei denen die ccRelaxation bereits aktiv ist. Auch führt ein mehrfaches Durchlaufen des zugehörigen Temperaturbereichs mit der DSC zu keinen Veränderungen. Dies liegt daran dass hier keine eingeschreckten Verspannungen relaxieren, sondern nur der Übergang zu einer Temperatur erfolgt, bei der die Relaxationszeit zur charakteristischen Zeit der Messung resonant wird. Die Vielzahl der auftretenden Relaxationen erschwert die Untersuchung, welchen Einfluss die Variation von Heizrate und Spektroskopiefrequenz hat. Dies zeigt sich auch beim Vergleich von DMA- und DSC-Messungen. Der deutliche Unterschied im mechanischen Verhalten zwischen bei niedrigem Druck synthetisierten Proben, die über eine cc-Relaxation und mehr charakteristische Längenskalen verfügen, und Hochdruckproben motiviert zu weiteren Untersuchungen. Eine noch genauere Aufklärung der chemischen Struktur, DES und DMA im empfindlichen, uniaxialen Zug-Modus könnten sich hier in Zukunft als sehr nützlich erweisen. 


\section{Literaturverzeichnis}

[AHL88] K. Ahlborn, Cryogenics 28, 234 (1988)

[AM05] M. Alcoutlabi, G. B. McKenna, J. of Phys. Cond. Mat. 17 (15), R461 (2005)

[ANG88] C. A. Angell, J. of Non-Cryst. Sol. 102, 205 (1988)

[ANG94] C. A. Angell, J. Phys.: Cond. Mat. 16, 5153 (2004)

[ARC96] A. Arbe, D. Richter, J. Colmenero, B. Farago, Phys. Rev. E 54 (4), 3853 (1996)

[AT74] C. A. Angell, J. C. Tucker, J. Phys. Chem. 78, 278 (1974)

[BBD97] M. Buback, M. Busch, T. Dröge, F.-O. Mähling, C. Prellberg, Eur. Polym. J. 33, 375 (1997)

[BEC06] F. Becker, "Untersuchung und Modellierung der integralen und kettenlängendifferenzierten Mikrostruktur von Hochdruckpolyethylen", Doktorarbeit Göttingen (2006)

[BER60] J. D. Bernal, Nature 185, 68 (1960)

[BK05] K. Binder, W. Kob, "Glassy materials and disordered solids", World Scientific, (2005)

[BNA93] R. Böhmer, K. L. Ngai, C. A. Angell, D. J. Plazek, J. Chem. Phys. 99 (5) 4201 (1993)

[BOX00] H. C. M. van Boxtel, "Modeling kinetics in the high-pressure free radical binary and ternary copolymerization of ethane with methyl acrylate and vinyl acetate as comonomers", Doktorarbeit Göttingen (2000)

[BS07] B. Steisel, persönliche Mitteilung mit Inhalt, der in der Doktorarbeit von B. Steisel diskutiert werden soll, erwarteter Zeitpunkt der Abgabe (2007)

[CC41] K. S. Cole, R. H. Cole, J. Chem. Phys. 9, 342 (1941)

[CG72] H. S. Chen, M. Goldstein, J. Appl. Phys. 43, 4 (1972)

[COW80] J. M. G. Cowie, J. Macromol. Sci.-Phys., 18 (4), 569 (1980)

[CSB04] A. I. Chumakov, I. Sergueev, U. van Bürck, W. Schirmacher, T. Asthalter, R. Rüffer, O. Leupold, W. Petry, Phys. Rev. Lett. 92, 245508 (2004)

[CUL78] B. D. Cullity, "Elements of X-Ray Diffraction", second edition, Addison-Wesley (1978)

[DC50] D. W. Davidson, R. H. Cole, J. Chem. Phys. 18, 1417 (1950)

[DC51] D. W. Davidson, R. H. Cole, J. Chem. Phys. 19, 1484 (1951)

[DE88] M. Doi, S. F. Edwards, "The theory of polymer dynamics", Oxford Science Publications, Clarendon Press (1988)

[DHT06] M. D. Demetriou, J. S. Harmon, M. Tao, G. Duan, K. Samwer, W. L. Johnson Phys. Rev. Lett.97, 065502 (2006)

[DJ04] M. D. Demetriou, W.L. Johnson, Acta Mat. 52, 3403 (2004)

[DJ05] M. D. Demetriou, W. L. Johnson, Scripta Mat. 52, 833 (2005)

[DON01] E. J. Donth, "The glass transition - Relaxation dynamics in liquids and disordered materials", Springer Verlag (2001) 
[DS01] P. G. Debenedetti, F. H. Stillinger, Nature 410, 259 (2001)

[DYR05] J. C. Dyre, Phys. Rev. E 72, 011501 (2005)

[EAN96] M. D. Ediger, C. A. Angell, S. R. Nagel, J. Phys. Chem. 100, 13200 (1996)

[ELL90] S. R. Elliot, "Physics of amorphous materials", second edition, Longman Scientific \& Technical (1990)

[FFM03] F. Faupel, W. Frank, M.-P. Macht, H. Mehrer, V. Naundorf, K. Rätzke, H. R. Schober, S. K. Sharma, H. Teichler, Rev. of Mod. Phys. 75 (1), 237 (2003)

[FLS77] R. P. Feynman, R. B. Leighton, M. Sands, "The Feynman lectures on physics", Band I-III, 6. Auflage, Addison Wesley (1977)

[FUL23] G. S. Fulcher, J. Am. Ceram. Soc. 8, 229 (1923)

[GÖT99] W. Götze, J. of Phys. Cond. Mat. 11 (10A), A1 (1999)

[GNE92] J. I. Goldstein, D. E. Newbury, P. Echlin, D. C. Joy, A. D. Romig, C. E. Lyman, C. Fiori, E. Lifshin, "Scanning electron microscopy and x-ray microanalysis", second edition, Plenum Press (1992)

[GÖT98] W. Götze, Cond. Mat. Phys. 1 (4), 873 (1998)

[GV97] H. Vogel, "Gerthsen Physik", 19. Auflage, Springer (1997)

[HAA94] P. Haasen, "Physikalische Metallkunde", 3. Auflage, Springer (1994)

[HAC03] J. Hachenberg, "Oberflächenwachstum laserdeponierter Polymerschichten”, Diplomarbeit Göttingen (2003)

[HAR06] T. Haramina, "Mechanical spectroscopy of polymers with reduced dimensions and increasing cross-linking degree", Doktorarbeit Göttingen (2006)

[HBP96] N. J. Heaton, R. Benavente, E. Pérez, A. Bello, J. M. Perena, Polymer 37 (17), 3791 (1996)

[HN67] S. Havriliak, S. Negami, Polymer 8, 161 (1967)

[IRÖ04] I. Rösner, "Relaxationsprozesse und Kristallisation im glasbildenden System $\mathrm{Pd}_{77} \mathrm{Cu}_{6} \mathrm{Si}_{17}$ ", Diplomarbeit Göttingen (2004)

[IRS95] B. Schrader, "Infrared and Raman Spectroscopy: Methods and Applications", $\mathrm{VCH}(1995)$

[JAN94] C. Janot, "Quasicrystals" in "Monographs on the physics and chemistry of materials 50", second edition, Oxford Science Publications (1994)

[JON81] H. Jones, "Experimental methods in rapid quenching from the melt" in "Treatise on materials science and technology: Volume 20 Ultrarapid quenching of liquid alloys", Academic press (1981)

[JS05] W. L. Johnson, K. Samwer, Phys. Rev. Lett. 95, 195501 (2005)

[KAH06] A. Kahl, “Ultraschallspektroskopie an dem amorphen System $\mathrm{Pd}_{40} \mathrm{Ni}_{40} \mathrm{P}_{20}$ ”, Diplomarbeit Göttingen (2006)

[KBB99] A. Kudlik, S. Benkhof, T. Blochowicz, C. Tschirwitz, E. Rössler, J. Mol. Struct. 479, 201 (1999)

[KKL05] S. Kalakkunnath, D. S. Kalika, H. Lin, B. D. Freeman, Macromolecules 38, 9679 (2005) 
[KS03] F. Kremer, A. Schönhals, "Broadband dielectric spectroscopy", Springer (2003)

[LKS82] G. Luft, R. Kämpf, H. Seidl, Die Angew. Makrom. Chem. 108(1708), 203 (1982)

[LL91] L. D. Landau, E. M. Lifschitz, "Lehrbuch der theoretischen Physik, Band VII, Elastizitätstheorie", 7. Auflage, Akademie Verlag Berlin (1991)

[LL00] P. Lunkenheimer, U. Schneider, R. Brand, A. Loidl, Contemp. Phys. 41, 15 (2000)

[LRJ03] J. Lu, G. Ravichandran, W.L. Johnson, Acta Mat. 51, 3429 (2003)

[LWU05] P. Lunkenheimer, R. Wehn, U. Schneider, A. Loidl, Phys. Rev. Lett. 95, 055702 (2005)

[LXG90] Li Xiao-Guang, J. Phys. D: Appl. Phys. 23, 368 (1990)

[MAT94] V. B. F. Mathot, "Calorimetry and thermal analysis of polymers", Hanser Publishers (1994)

[MEN99] K. P. Menard, "Dynamic mechanical analysis: a practical introduction", CRC Press (1999)

[MRW67] N. G. McCrum, B. E. Read, G. Williams, "Anelastic and dielectric effects in polymeric solids", John Wiley \& Sons (1967)

[MWB99] A. Masuhr, A. Waniuk, R. Busch, W. L. Johnson, Phys. Rev. Lett. 82 (11), 2290 (1999)

[PAN92] K. Panten, "Radikalische Hochdruck-Terpolymerisation von Ethen, Acrylnitril und Vinylacetat", Doktorarbeit Göttingen (1992)

[PE94] Perkin-Elmer, "User Manual 7 Series / Unix DMA 7 / DMA 7e", manual version F, Perkin-Elmer (1994)

[PPS00] J. M. Pelletier, J. Perez, J. L. Soubeyroux, J. of Non-Cryst. Sol. 274, 301(2000)

[PRI99] T. Pritz, J. o. Sound a. Vibration 228 (5), 1145 (1999)

[RAM94] R. Rambousky, "Dynamisch-mechanische Eigenschaften neuer ternärer und mehrkomponentiger Gläser", Diplomarbeit Augsburg (1994)

[RIC02] D. Richter, "Polymer dynamics", Kap. C2 in "Soft matter: complex materials on mesoscopic scales", 33. IFF-Ferienkurs, Forschungszentrum Jülich (2002)

[RÖS04] P. Rösner, "Mechanische Relaxation in komplexen Fluiden”, Doktorarbeit Göttingen (2004)

[RSL04] P. Rösner, K. Samwer, P. Lunkenheimer, Europhys. Lett. 68, 226 (2004)

[SGS02] A. Schönhals, H. Goering, Ch. Schick, J. of Non-Cryst. Sol. 305, 140 (2002)

[SGS05] A. Schönhals, H. Goering, Ch. Schick, B. Frick, R. Zorn, J. of Non-Cryst. Sol. 351, 2668 (2005)

[SIL99] H. Sillescu, J. of Non-Cryst. Sol. 243 (2), 81 (1999)

[SK03] A. Serghei, F. Kremer, Phys. Rev. Lett. 91 (16), 165702 (2003)

[SL83] V. D. Scott, G. Love, "Quantitative electron-probe microanalysis", Ellis Horwood Limited (1983) 
[SPE92] L. H. Sperling, "Introduction to physical polymerscience", second edition, Wiley-Interscience (1992)

[STI88] F. H. Stillinger, J. Chem. Phys. 88, 7818 (1988)

[STR77] L. C. E. Struik, "Physical aging in amorphous polymers and other materials", Doktorarbeit, TH Delft (1977) oder Elsevier Science, ISBN: 0444416552 (1978)

[STU04] B. Stuart, "Infrared spectroscopy: Fundamentals and applications", John Wiley \& Sons (2004)

[SVA03] C. Svanberg, J. Appl. Phys. 94 (6), 4191 (2003)

[TAM26] G. Tammann, W. Hesse, Z. Anorg. Allg. Chem. 156, 245 (1926)

[TEI05] H. Teichler, Phys. Rev. E 71, 031505 (2005)

[TS85] S. S. Tsao, F. Spaepen, Acta Met. 33 (5), 881 (1985)

[VLZ05]～K. Vollmayr-Lee, A. Zippelius, Phys. Rev. E 72, 041507 (2005)

[VOG21] H. Vogel, Phys. Z. 22, 645 (1921)

[WAR83] I. M. Ward, "Mechanical properties of solid polymers", Second Edition, WileyInterscience (1983)

[WAS80] Y. Waseda, "The structure of non-crystalline materials", McGraw-Hill Inc. (1980)

[WBZ02] L. Wang, X. Bian, J. Zhang, "Structural simulation of clusters in liquid $\mathrm{Ni}_{50} \mathrm{Al}_{50}$ alloys”, Modelling Simul. Mater. Sci. Eng. 10, 331 (2002)

[WEI98] M. Weiß, "Mechanische Eigenschaften und thermische Stabilität tief unterkühlter Metallschmelzen im Bereich der Glastemperatur", Doktorarbeit Augsburg (1998)

[WITT99] L. Wittkowski, "Experimentelles Studium und Modellierung der radikalischen Hochdruck-Copolymerisation von Ethen und (Meth)Acrylsäure”, Doktorarbeit, Göttingen 1999

[WU03] C.-S. Wu, "Handbook of size exclusion chromatography and related techniques", second edition, chromatographic science series 91, Marcel Decker (2003)

[ZAR91] J. Zarzycki, "Glasses and the vitreous state", Cambridge University Press (1991)

[ZOR03] R. Zorn, J. of Phys. Cond. Mat. 15 (23), R1025 (2003)

[ZSJ06] M. Zink, K. Samwer, W. L. Johnson and S. G. Mayr, Phys. Rev. B 73, 1772203 (2006) und Phys. Rev. B 74, 012201 (2006) 


\section{Danksagung}

Mein besonderer Dank gilt Prof. Dr. K. Samwer für die Betreuung meiner Arbeit. Er hat mich sehr gefördert und auch im richtigen Maße gefordert und war dabei stets offen für andere Meinungen, großzügig und interessiert. Besonders wertvoll waren für mich die vielen Diskussionsrunden auf der einen Seite und die Möglichkeit mich frei entfalten zu dürfen auf der anderen.

Bei Prof. Dr. M. Buback möchte ich mich dafür bedanken, dass es mich so freundlich im Rahmen des Graduiertenkollegs 782 als Second Supervisor betreut hat. Die Herstellung der Copolymer-Proben unterlag seiner Obhut, und er war mir ein wertvoller Ratgeber in Fragen der Chemie.

Für die Finanzierung meiner Promotion und der zahlreichen Dienstreisen danke ich dem Graduiertenkolleg 782 der DFG, vertreten durch den engagierten Prof. Dr. M. Suhm und die tatkräftige Frau J. Kupferschmid. Durch den engen Verbund entstanden wichtige Synergieeffekte bei der Copolymer-Synthese und -Analyse. In diesem Rahmen gilt mein Dank auch Prof. Dr. C. Griesinger, Dr. M. Baldus und Robert Schneider für die Durchführung und Deutung der NMR-Untersuchungen. Die Projektwoche in der Arbeitsgruppe von Prof. Dr. U. Diederichsen unter der Anleitung von Dr. Nicola Diezemann erlaubte mir den Blick über den Tellerrand in die wundersame Welt der Peptide. Auf dem kurzen Dienstweg standen mir stets das gesammelte Wissen und die experimentellen Techniken des Kollegs offen, vereint bei der Ringvorlesung, den Seminarausflügen und Gemeinschaftsabenden.

Inspirierend waren für mich die Diskussionen mit Prof. Dr. A. Angell, der uns als Gast mehrfach beehrte. Danke für die vielen Anregungen.

Sehr bereichernd war für mich die wissenschaftliche Diskussion mit Prof. Dr. H.-U. Krebs und der von ihm geleiteten Polymer-Runde. Daten, die darin Bestand haben, können getrost publiziert werden.

Ohne Björn Steisel stünde diese Arbeit nur auf einem Bein. Seinem Geschick unterstanden die Synthese der Copolymere und deren chemische Analyse. Mir hat die Zusammenarbeit mir ihm stets Spaß gemacht und ich habe viel von ihm gelernt. Danke vielmals!

Für den guten Start in die Glasphysik danke ich Dr. Peter Rösner und seiner Frau Iris. Peter hatte bei allen Fragen ein offenes Ohr und zusammen mit Iris fiel mir die Einarbeitung in die DMA leichter.

Ich danke Dr. M. Weiß für die Einweisung in die DMA. Bei der Inbetriebnahme der DMA und bei der Versorgung mit den anfangs oft benötigten Ersatzteilen haben mir Herr K.-H. Bücher und Herr W. Weigelt von PerkinElmer sehr geholfen. Vielen Dank für die vielen Kniffe und Tricks!

Ein großes Dankeschön sage ich der Glasgruppe, insbesondere Maria Tschuschke, Annelen Kahl, Dennis Bedorf und Stefan Buschhorn. Man versteht die eigenen Messungen am besten im Gespräch mit Gleichgesonnenen. Die Zusammenarbeit hat mir viel Spaß gemacht. Danke auch für das Korrekturlesen! 
Uta Bete, Katrin Gehrke, Carsten Mahn und der Crew der Feinmechanikwerkstatt möchte ich ganz herzlich für die große Hilfe bei der Realisierung meiner Experimente danken. Ein Koch ist nur so gut wie seine Zutaten!

Meinen Zimmerkollegen Sebastian Vauth und Christian Vree danke ich für das freundschaftliche Arbeitsklima. Ich habe mich bei ihnen sehr wohl gefühlt.

Für die schöne Zeit im I. Physikalischen Institut danke ich allen Mitarbeitern und Kollegen. Die gemeinsame Kaffeepause und die Grillabende haben mir stets Spaß gemacht. Ein echtes Highlight waren für mich die Seminare in Serneus und unsere anderen Ausflüge, bei denen es besonders abends nie langweilig wurde.

Meinen Freunden möchte ich für die nötige Ablenkung an vielen lustigen Abenden danken. Der Weg mit ihnen war schön und wird es hoffentlich auch weiter sein.

Meinen Eltern danke ich dafür, dass sie mir mein Studium ermöglicht haben und für alles, das sie mir mit auf den Weg gaben. Ich konnte stets auf sie und ihren Glauben an mich bauen.

Meiner Frau Inga danke ich für ihre Geduld mit mir während der Zeit meiner Doktorarbeit. Mit ihr waren die drei Jahre erst schön. „Und wenn ich prophetisch reden könnte und alle Geheimnisse wüsste und alle Erkenntnisse hätte, (...) hätte aber die Liebe nicht, wäre ich nichts." [1 Kor 13,2] 


\section{Lebenslauf}

\section{Persönliche Daten}

Name: $\quad$ Jörg Hachenberg

Adresse: $\quad$ Max-Born-Ring 29a

37077 Göttingen

Email: $\quad$ joerg.hachenberg@phys.uni-goettingen.de

Geburtsdatum: 21.12.1977

Nationalität: Deutsch

Familienstand: Verheiratet mit Inga Hachenberg, geb. Lahme, keine Kinder

\section{Ausbildung}

1990-1997

Seit 1998

WS 2000/01

2003

2003-2006
Campe-Gymnasium Holzminden, abgeschlossen mit dem Abitur (Note: 1,8) Studium der Physik an der Georg-August-Universität Göttingen

Auslandssemester an der Université Claude Bernard Lyon 1 in Frankreich im Erasmus-Programm

Diplom: „Oberflächenflächenwachstum laserdeponierter Polymerschichten“ (Note: Sehr gut)

Stipendiat der DFG im Graduiertenkolleg 782

\section{Berufserfahrung}

1994 Schulisches Berufspraktikum am Amtsgericht Holzminden

1997-1998 Grundwehrdienst beim Pionierbatallion 1, Holzminden

1998-2001 Ferienjobs im Rahmen eines Ausbildungsvertrags bei der Point GmbH \& Co. KG, Holzminden: Produktion und Konfiguration von PC-Systemen und Tätigkeit im Kundenservice

Seit 2002 Studentische / wissenschaftliche Hilfskraft an der Georg-August-Universität Göttingen: Übungsgruppenleiter in Physik 1, Festkörperphysik und Versuchsbetreuer im Fortgeschrittenenpraktikum

\section{Weiterbildungen}

$2000 \quad$ European DPG-Erasmus Physics School Bad Honnef: "Nuclear Methods in Material Science“"

2002 IFF Ferienschule Jülich: "Soft Matter"

2004 IFF Ferienschule Jülich: "Physics meets Biology"

2005 Universität Áalborg, Dänemark: "Ph.D. Course in Protein Fibrillation and Aggregation" 


\section{Publikationen}

2004

J. Hachenberg, C. Streng, E.Süske, S. Vauth, S. G. Mayr, H.-U. Krebs, K. Samwer: "Kinetic roughening of laser deposited polymer films: Crossover from single particle character to continuous growth" Phys. Rev. Lett. 92, 246102 (2004)

2006 P. Rösner, J. Hachenberg, K. Samwer, R. Wehn, P. Lunkenheimer, A. Loidl, E. Süske, T. Scharf and H.-U. Krebs: "Comparison of mechanical and dielectric relaxation in laser-deposited poly(methyl methacrylate) films" New J. of Phys. 8, 89 (2006)

2006 J. Hachenberg, K. Samwer: "Indications for a slow $\beta$-relaxation in a fragile metallic glass"

J. of Non-Cryst. Sol (2006), in press: doi:10.1016/j.jnoncrysol.2006.01.143

\section{Vorträge auf Konferenzen}

2003

Frühjahrstagung der Deutschen Physikalische Gesellschaft, Dresden

2005

Edgar-Lüscher-Seminar, Serneus, Schweiz

2005

5th International Discussion Meeting on Relaxation in Complex Systems,

Lille, Frankreich

2006

Frühjahrstagung der Deutschen Physikalische Gesellschaft, Dresden

Seit 2003

Diverse Posterpräsentationen auf Konferenzen und Seminaren

Göttingen, 21.09.2006 